\title{
Molecular Simulations of Zeolites: Adsorption, Diffusion, and Shape Selectivity
}

\author{
Berend Smit*
}

Department of Chemical Engineering, University of California, Berkeley, California 94720-1462, Van't Hoff Institute for Molecular Sciences, University of Amsterdam, Nieuwe Achtergracht 166, 1018 WV Amsterdam, The Netherlands, and Centre Européen de Calcul Atomique et Moléculaire (CECAM), Ecole Normale Supérieure, 46 Allée d'Italie, 69007 Lyon France

\author{
Theo L. M. Maesen \\ Chevron, Energy Technology Company, 100 Chevron Way, Richmond, California 94802-0627
}

Received August 8, 2002

\section{Contents}

1. Introduction

2. Basic Simulation Techniques

2.1. Molecular Mechanics

2.2. Molecular Dynamics

2.3. Monte Carlo Simulations

2.4. Simulating Free Energies

2.5. Dual Control Volume Grand Canonical Molecular Dynamics

2.6. Kinetic Monte Carlo

2.7. Ab Initio Molecular Dynamics

2.8. Hybrid Techniques: The QM/MM Approach

3. Advanced Simulation Techniques

3.1. Configurational-Bias Monte Carlo

3.2. Rare Event Simulations

4. Molecular Models: Inter- and Intramolecular Potentials

4.1. The Zeolite Structure

4.2. Adsorbate-Adsorbate Interactions

4.3. Zeolite-Adsorbate Interactions

4.4. Cation-Zeolite-Adsorbate Interactions

4.4.1. Cation-Zeolite Interactions

4.4.2. Cation-Adsorbate Interactions

5. Adsorption of Hydrocarbons in Zeolites

5.1. Introduction

5.2. Theoretical Aspects

5.2.1. Adsorption Thermodynamics

5.2.2. Adsorption Isotherms

5.3. Pure Component Adsorption

5.3.1. Hydrocarbons MFI

5.3.2. Other Zeolites

5.3.3. Flexible Lattice

5.3.4. Cation Effects

5.4. Adsorption of Mixtures

5.4.1. Adsorption Mechanisms

5.4.2. Mixtures Adsorption Studies

5.4.3. Effect of Cations

6. Diffusion in Zeolites

6.1. Introduction
4126

4127

4127

4127

4129

4130

4131

4131

4132

4132

4132

4132

4134

4135

4135

4136

4136

4138

4138

4139

4139

4139

4139

4139

4140

4141

4141

4143

4145

4145

4146

4146

4146

4148

4148

4148

* To whom correspondence should be addressed: Department of Chemical Engineering, University of California, Berkeley, 101B Gilman Hall \#1462, Berkeley, CA 94720-1462.

6.2. Theoretical Aspects 4149

6.2.1. Which Diffusion Coefficient? 4149

6.2.2. Multicomponent Diffusion 4150

6.3. Computational Aspects 4150

6.3.1. Molecular Dynamics Simulations 4150

6.3.2. Rare Event Simulations and Kinetic Monte 4151 Carlo

6.3.3. Effect of a Flexible Lattice 4152

6.4. A Simple Lattice Model 4153

6.4.1. Temperature Dependence 4153

6.4.2. Loading Dependence 4153

6.4.3. Correlations 4155

6.5. Diffusion of Simple Pure Components 4155

6.5.1. Loading Dependence 4156

6.5.2. Methane in MFI 4157

6.5.3. Methane in Other Zeolites 4159

6.6. Diffusion of Long Chain Hydrocarbons 4160

6.6.1. Hydrocarbons in MFI 4160

6.6.2. Resonance Diffusion 4161

6.6.3. Single-File Diffusion 4163

6.6.4. Understanding Chain Length Effects 4164

6.7. Mixture Diffusion 4165

6.7.1. In-Silico Design of a Separation Process 4165

6.8. Toward Real-Life Zeolites 4165

7. Toward an Understanding of Shape Selectivity 4166

7.1. Introduction 4166

7.2. Conventional Hydroconversion Mechanisms 4166

7.2.1. Basic Mechanism 4166

7.2.2. Traditional Shape Selectivity 4168

7.2.3. Transition State Shape Selectivity 4168

7.2.4. Reactant Shape Selectivity 4169

7.2.5. Product Shape Selectivity 4169

7.2.6. Exterior Surface Shape Selectivity 4169

7.2.7. Other Forms of Shape Selectivity 4169

7.3. New Mechanism: The Free Energy Landscape 4169

7.3.1. Free Energy Landscape $\quad 4170$

7.3.2. Conventional Shape Selectivity $\quad 4170$

7.3.3. Transition State Selectivity 4171

7.3.4. Reactant Shape Selectivity 4174

7.3.5. Product Shape Selectivity 4176

7.3.6. Free Energies and Diffusion: Partial 4176 Adsorption

7.4. In Silico Screening

4177 


\section{Outlook \\ 9. Acknowledgments \\ 10. References}

\section{Introduction}

Zeolites are intriguing materials. The basic building blocks are corner sharing $\mathrm{TO}_{4}$ units, where the T-atoms usually refer to $\mathrm{Si}, \mathrm{Al}$, or in some cases also $\mathrm{P}$. These tetrahedrals can form different types of units, such as 6-rings, 8-rings, or 12rings. These are, so-called, secondary building units of different types of cylinders or cages. The importance of these materials is that these cylinders or cages form a network of pores in the zeolite crystal. Over 180 different structures have been made, all with more or less the same chemical composition but with different pore topologies (see Figure 1 for some examples). ${ }^{1}$ The fact that these pores are accessible from the outside and the unique stability of these materials make them interesting for many different applications. $^{2}$

Most of these applications rely on the fact that the pores have dimensions that are comparable with the size of the molecules that can be adsorbed. Zeolite design involves selecting the optimal pore topology for a give application. As the dimensions of these pores are comparable, the thermodynamic properties of the adsorbed molecules are very different from the bulk properties. For example, the diffusion coefficient of methane can vary as much as $6-10$ orders of magnitude (!) depending on the pore topology. Similarly, at a given pressure of methane, the number of adsorbed molecules varies enormously from one structure to another.

Zeolites are an important class of molecular sieves in which separations are obtained by selecting the pore size such that in mixtures molecules that are too large are being blocked. ${ }^{4}$ Competitive adsorption makes $\mathrm{Ca}^{2+}$ ions much stronger adsorbing in zeolites compared to $\mathrm{Na}^{+}$, which makes zeolites an important component in laundry powders to reduce the water hardness. In this review we focus on zeolites that are used in petrochemical applications as catalysts. Many examples of hydrocarbon molecules adsorbed and converted by the zeolites can be found in the literature. ${ }^{5-7}$ The importance of this application is best illustrated in Figure 2, which shows that the introduction of zeolites and subsequent further process improvements made the production of gasoline $10 \%$ more efficient. This $10 \%$ may not sound too impressive, but if one multiplies this number with the enormous volumes of gasoline that have been and will be produced, this number translates into an enormous energy savings.

From a scientific point of view, zeolites are challenging. As most of the activity occurs inside the material, it is very difficult to carry out experiments that provide us information at the molecular level. Understanding a reaction inside the pores requires knowledge on the adsorption of the reactants, on the diffusion of these molecules to the active site, on the chemical conversion at the active site, and subsequently on the products diffusing away from the active site and finally desorbing from the zeolite. Each of these steps may have an influence on the reaction rates and products that are formed. That the molecules of interest are inside a material makes it much more difficult to obtain information at the molecular level. In this respect molecular simulations nicely complement the experimental efforts. ${ }^{9,10}$

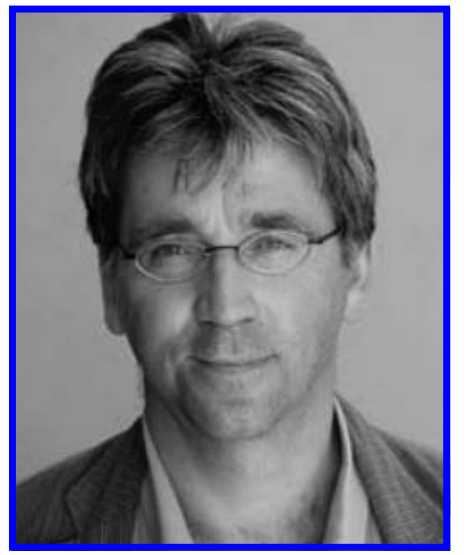

Berend Smit is a native of Deventer, The Netherlands. He obtained his M.Sc. in Chemical Engineering in 1987 and his M.Sc. in Physics in 1988, both from the Technical University in Delft. He obtained a Ph.D. in Chemistry in 1990 from Utrecht University. From 1988 to 1997, he worked as a researcher at Shell Research in Amsterdam. In 1997, he returned to academia as Professor of Computational Chemistry at the University of Amsterdam. In 2005, he was elected as director of CECAM in Lyon France. Since 2007, he has been Professor of Chemical Engineering and Professor of Chemistry at the University of California, Berkeley. His research interests include the development and application of advanced molecular simulation techniques.

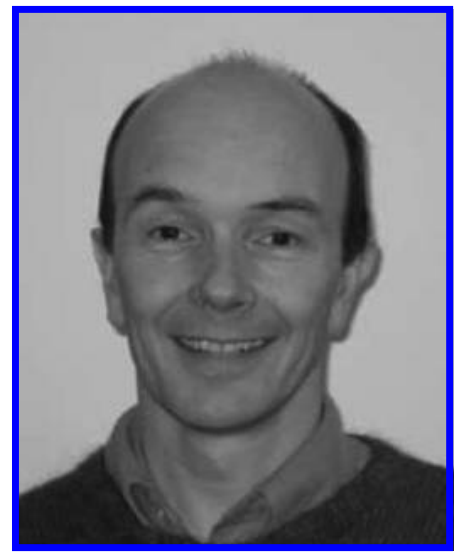

Theo Maesen is a native of Stein, The Netherlands. He obtained his B.Sc. in Organic Chemistry and his M.Sc. in Catalysis at Leiden University, The Netherlands. After a Ph.D. in Catalysis at the Technical University Delft (1990), he joined Shell at its Reearch and Development Center in Amsterdam. He obtained his M.Sc. in Chemical Engineering at the University Twente in 1994. He joined Chevron at its Energy and Technology Center in 2000. His research interests include hydroprocessing and catalytic cracking fundamentals, particularly as they relate to adsorption, diffusion, and thermodynamics.

Understanding shape selectivity requires understanding the kinetics (diffusion) and thermodynamics. Therefore we start our discussion with the applications of molecular simulation in adsorption, followed by diffusion, and finally shape selectivity. We focus on the adsorption of simple hydrocarbons such as linear and branched alkanes. In each topic we try to demonstrate how simulations have contributed to a better qualitative and quantitative understanding of these materials. For example, we try to qualitatively explain why certain zeolites specifically adsorb one type of molecules while in other structures the selectivity is reversed. In addition to this can we develop a molecular simulation model that can quantitatively predict these properties for the molecules of interest at the conditions of interest. We feel that sufficient progress has been made to write a review in which we try to give a comprehensive view of adsorption, diffusion, and shape selectivity. In this review we focus on classical force- 


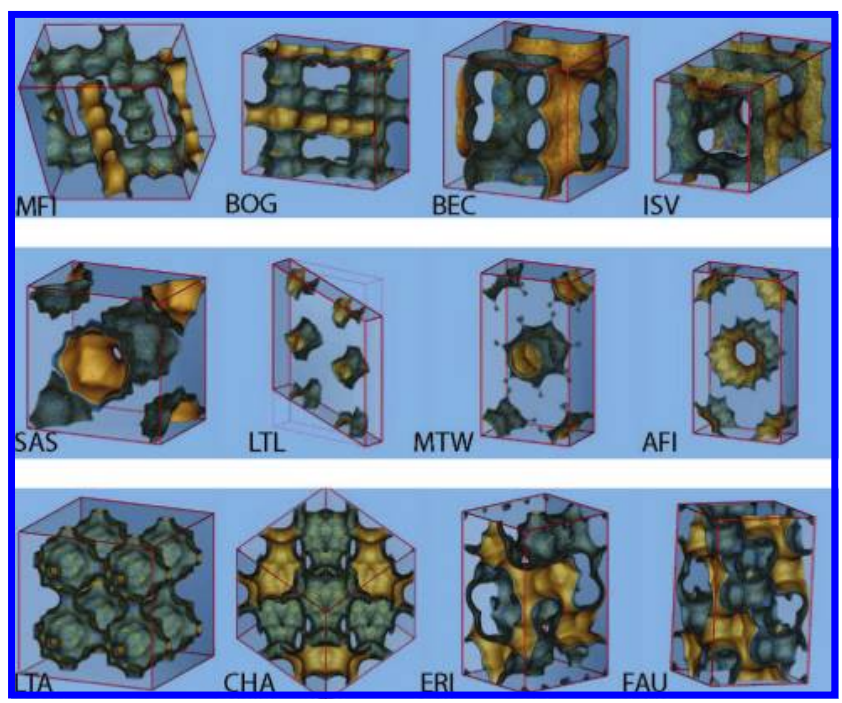

Figure 1. Examples of some zeolite structures: three-dimensional networks (top), one-dimensional channels (middle), and cagelike structures (bottom). The three letter codes refer to the framework type codes that have been assigned by the Structure Commission of the International Zeolite Association. Used with permission from ref 3. Copyright 2006 American Chemical Society.

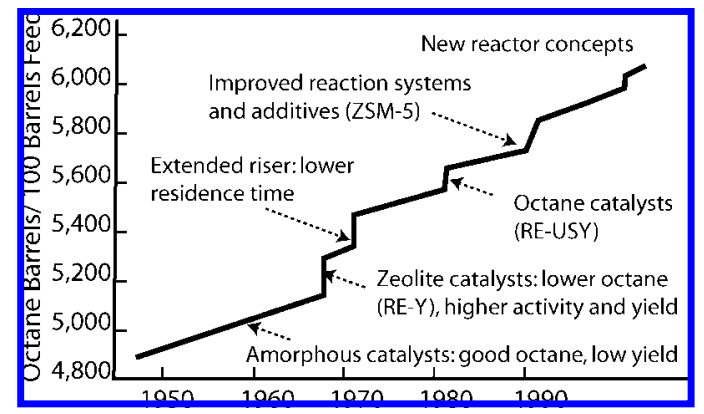

Figure 2. Effect of the introduction of zeolites on the number "octane" barrels that can be produced from oil feed stocks (figure based on the information provided in ref 8).

field-based methods. Density functional theory based calculations are beyond the scope of this work.

Our review is written from a computational perspective and focused on the contribution of molecular simulations to our understanding of these topics. This very limited focus should therefore by no means be interpreted as a lack of importance or progress on the experimental side. But it does explain why, instead, we start this review with an extensive discussion of the simulation techniques that have been developed to make these types of simulations possible and a discussion of the different molecular models (force fields) that have been proposed to simulate molecules adsorbed in zeolites.

\section{Basic Simulation Techniques}

The principles of molecular simulations are simple. A system is modeled by describing the interactions between the atoms. An appropriate molecular simulation technique is used to link these interactions at the molecular level to macroscopic quantities that are accessible experimentally.

In this section we will outline the specific requirements that guide the selection of the appropriate simulation for studying diffusion and adsorption in zeolites. Before discussing the specific aspects for zeolites, a short description of the most common simulation techniques is given. A detailed description of these methods can be found in one of the textbooks on molecular simulations. ${ }^{1-14}$

\subsection{Molecular Mechanics}

One of the first molecular simulation techniques that have been applied to zeolites is molecular mechanics (MM). In an $\mathrm{MM}$ or docking calculation the minimum energy configuration of a molecule adsorbed in a zeolite is calculated. Calculating the minimum energy in a high dimensional space is nontrivial, and one must ensure that the calculation does not stop at a local minimum. Many techniques to find this energy minimum have been developed, which have been generalized to arbitrary molecules. Many commercial or public domain molecular simulation packages can be found which can be used to perform an MM simulation.

From a practical point of view, a molecular mechanics simulation is very cheap; the end result is a single conformation, i.e., the positions of all atoms of the molecule in the zeolite that give the lowest energy. It is, however, more difficult to compare this molecular mechanics results with experimental data. Statistical thermodynamics tells us that this minimum energy conformation corresponds exactly to the conformation of the system at $0 \mathrm{~K}$. If we increase the temperature, entropy effects will play a role and many other conformations would contribute to the thermodynamic properties. It is not possible to use this minimum energy for comparison with, say, heats of adsorption data from experiments. Molecular mechanics simulations are therefore mainly used to estimate whether or not a molecule can fit in a particular zeolite.

A more sophisticated form of molecular mechanics simulations is to compute the minimum energy as a function of the position of the molecule in the zeolite. For example, suppose we are interested in a molecule diffusing through a zeolite which consists of cages separated by narrow windows. A molecular mechanics simulation would show us, for example, an energy barrier if the molecule is moved from one cage to another through the window. These types of calculations do give an estimate whether or not a molecule can diffuse through a zeolite channel, but different types of simulations are required to compute a diffusion coefficient.

Even with the perfect intermolecular interactions, molecular mechanics can, at best, approximate the experimental quantities. Unfortunately, there is no systematic way to converge to the desired quantities. Therefore a molecular mechanics simulation mainly yields qualitative information, which can be very useful for a particular application.

\subsection{Molecular Dynamics}

In molecular dynamics (MD) simulations we use interand intramolecular interactions to compute the forces on the molecules. Once these forces are known, Newton's equations of motion can be solved numerically and the positions and velocities of the molecules can be computed for the next time step. This procedure can be repeated until we have simulated the system sufficiently long such that the thermodynamic and transport properties of the system can be computed.

The main limitation of a molecular dynamic simulation is CPU-time. If we would have an infinite amount of CPUtime available, many of the issues discussed next would not play any role. For example, we would be able to use a flexible zeolite or couple the zeolite to a large reservoir of 
molecules that adsorb. In fact, most of the techniques discussed in the next section would be completely obsolete. However, in practice CPU-time limitations impose important limitations on the systems that can reliably be simulated. Even if the enormous increase in CPU-power, which we have seen in last decades, continues, it would take many decades before these special techniques could be replaced by a straightforward molecular dynamics simulation.

Let us consider a system of $N$ particles that interact via an intermolecular pair potential $u(r)$. Let us denote the configuration of the system by $\Gamma^{N}=\mathbf{r}_{1}, \mathbf{r}_{2}, \ldots, \mathbf{r}_{N}$, where $\mathbf{r}_{i}$ is the vector describing the position of particle $i$. The total energy of the system is given by

$$
U\left(\Gamma^{N}\right)=\sum_{i>j} u\left(r_{i j}\right)
$$

where $r_{i j}$ is the distance between particles $i$ and $j$, and $u(r)$ is the potential energy between two particles. In the above equation it is assumed that the interactions are pairwise additive. The more general case will include three- and higher body interactions. From this potential the forces on all particles are computed and Newton's equations of motion are solved using one of the conventional numerical integration schemes. Details on the computational aspects related to these integration schemes can be found in one of the molecular simulation books. The calculation of the force on all particles involves a summation over all pairs of particles. This is the most expensive part of a simulation. Without any computational tricks, the cost of the calculation increases with $N^{2}$ as we increase the number of particles $N$. Methods have been developed to reduce the particle number dependence to an order $N$ algorithm.

Similar to a molecular mechanics calculation, a molecular dynamics algorithm is relatively easy to generalize to arbitrary molecules, and one can find reliable public domain and commercial packages with state of the art molecular dynamics software. Nevertheless, the use of molecular dynamics in the context of simulating the adsorption and diffusion is not simply a question of obtaining these codes and running them. For example, simulations of zeolites usually involve many atoms. Even if the zeolite is fully loaded, the majority of the atoms belong to the zeolite. Therefore, significant gains in CPU-time can be obtained if we assume that the zeolite is rigid, i.e., the atoms of the zeolites are fixed at their crystallographic positions. In section 5.3.3 we will discuss whether this assumption is a realistic approximation of the real system. Here, we focus on the computational consequences of this assumption.

If there are no external forces working on the system, the total energy is conserved in a molecular dynamics simulation. We therefore perform a simulation in which the energy, $E$, number of particles, $N$, and the volume, $V$ are imposed. A molecular dynamics simulation therefore samples the microcanonical ensemble. Experiments, however, are usually performed at constant temperature $(N V T$ or canonical ensemble) rather than constant energy. Methods have been developed to simulate a system at constant temperature. ${ }^{12}$ There are two approaches to perform a molecular dynamics simulation at constant temperature. The first approach, developed by Andersen, ${ }^{15}$ is a stochastic approach, in which each particle has a probability to collide with a heat bath, the so-called Andersen thermostat. After such a collision the particle is given a new velocity drawn from the Maxwell distribution corresponding to the imposed temperature. An

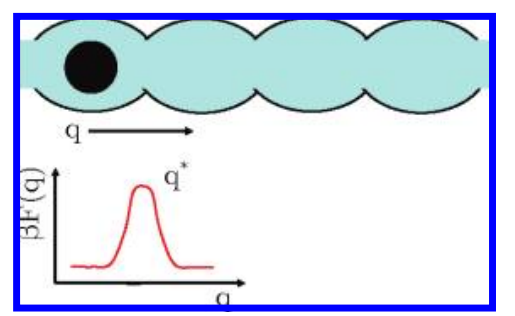

Figure 3. Schematic picture of a single molecule diffusing in a one-dimensional pore (top) and the free energy $F$ of this molecule as a function of the position, $q$ (bottom); in the cages the free energy is low, and in the window region, indicated by $q^{*}$, we observe a high free energy. The free energy is in units of $k_{\mathrm{B}} T(=1 / \beta)$.

alternative method is to extend the number of degrees of freedom. In addition to the $3 N$ positions and $3 N$ momenta, characterizing the state of the system, a new variable $s$ with associated momentum $p_{s}$ is introduced. The variables are coupled and an extended ensemble is formed. Nosé ${ }^{16}$ has proved that this coupling can be such that, if these new equations of motion are solved, the subsystem samples the $N V T$ ensemble. The coupling in the Nosé-Hoover method ${ }^{17}$ corresponds to rescaling of the momenta.

Whereas both methods sample the canonical ensemble correctly, both methods do change the dynamics of the system in an artificial way. ${ }^{12}$ Therefore, these constant temperature molecular dynamics simulation methods may lead to incorrect results for the dynamics properties. Often it is assumed that the perturbation in the Nosé-Hoover scheme is much less severe compared to that of the Andersen method and therefore yields a better approximation of the true dynamic quantities. ${ }^{12}$ Therefore, in bulk liquids or gases, if one is interested in computing diffusion coefficients at a particular temperature $T$, an $N V T$ simulation is used to equilibrate the system at the desired temperature and subsequently the ensemble is switched to $N V E$ if a diffusion coefficient needs to be computed. Such a procedure can also be used if we simulate a zeolite using a flexible lattice. However, if we simulate a rigid zeolite, one has to be very careful to compute diffusion coefficients.

Let us assume we simulate the one-dimensional zeolite pore shown in Figure 3. Often we are interested in the limit of very low loading, for example to compute the selfdiffusion coefficient at zero loading, which implies that the adsorbed molecules are very far apart and do not interact with each other. We can therefore focus on a single particle. In the $N V E$ ensemble a particle can only change its total energy if it collides with another adsorbed molecule. If we keep the positions of the zeolite particles fixed, a collision with a zeolite atom changes the momentum of the adsorbed molecule but not its total energy. Suppose that our molecule can only move in one dimension and it feels the potential energy shown in Figure 3. If the initial energy of our molecule is less than the maximum of the potential energy, then the molecule will oscillate around the minimum of the potential until it collides with another molecule and picks up sufficient kinetic energy to take the barrier. In addition, once it has taken the barrier, because of its ballistic movement, it will continue to move with a uniform average speed until it hits again another adsorbed molecule. If we would simulate a flexible lattice, then the adsorbed molecule could also change its total energy via a collision with one of the atoms of the zeolite. Clearly, the dynamics in the case of a rigid zeolite in the limit of infinite dilution can be very unrealistic if one uses the $N V E$ ensemble. 
Whereas in an $N V E$ simulation the energy only changes after a collision, if one uses the $N V T$ ensemble, the total energy of a particle changes during the simulation because of the action of the thermostat. Hence, the fluctuations in the total energy can cause the particle to cross a barrier. The Nosé-Hoover thermostat couples to the entire system and therefore gives a very small perturbation for each particle. In the case of adsorption in a zeolite, the Andersen thermostat might be more realistic; an adsorbed molecule only changes its total energy because of (inelastic) collisions with the zeolite atoms. ${ }^{18}$ Ideally, this frequency of collision with these atoms should be matched with the Andersen thermostat. Surprisingly, a systematic investigation to compare these differences has not been made, and therefore it is not trivial to make a comparison of the results obtained by the various methods. That the flexibility of the lattice can have a very significant effect has been illustrated by Jakobtorweihen et al., ${ }^{19}$ who showed that in the case of diffusion of methane in carbon nanotubes the diffusion coefficient in a rigid tube is 2 orders of magnitude higher than that in a tube in which the atoms are allowed to move.

\subsection{Monte Carlo Simulations}

In a molecular dynamics simulation the evolution of the system is simulated. Statistical thermodynamics assumes that if we simulate such a system at constant temperature sufficiently long, the probability that we find a particular configuration is given by the Boltzmann distribution

$$
P_{N V T}\left(\Gamma^{N}\right) \propto \exp \left[-\beta U\left(\Gamma^{N}\right)\right]
$$

where $\beta=1 /\left(k_{\mathrm{B}} T\right)$ and $U$ is the intermolecular potential. In a Monte Carlo (MC) simulation, a Markov chain consists of a sequence of configurations, where the probability of finding a particular configuration in this chain is given by the Boltzmann equation. In practice, such a chain is generated by performing attempts to generate a new configuration, for example, by displacing a randomly selected particle and accepting or rejecting this move according to an appropriate acceptance rule. This acceptance rule can be derived from the condition of detailed balance ${ }^{12}$

$$
\operatorname{acc}(o \rightarrow n)=\min (1, \exp \{-\beta[U(n)-U(o)]\})
$$

This equation shows that the probability of accepting/ rejecting depends on the energy difference between the old $(o)$ and the new $(n)$ conformation. The expensive part in a Monte Carlo simulation is the calculation of this energy difference. In this respect the Monte Carlo technique is similar to molecular dynamics and the CPU-limitations are identical. However, in a molecular dynamics simulation, the best one can do is to mimic nature as realistically as possible. If in nature diffusion coefficients are very small, this will be reflected in a molecular dynamics simulation by a very long simulation time, since it takes very long before the molecules have diffused sufficiently far that a new statistically independent configuration can be sampled. In a Monte Carlo simulation one does not have to follow the natural path; a new configuration can be generated in such a way that all diffusion barriers will be avoided.

Let us consider the experimental setup to measure adsorption isotherms; we would like to measure the number of adsorbed molecules as a function of the pressure of the gas or liquid that is in contact with the zeolite. Experimentally, the most common system is a zeolite in a container that

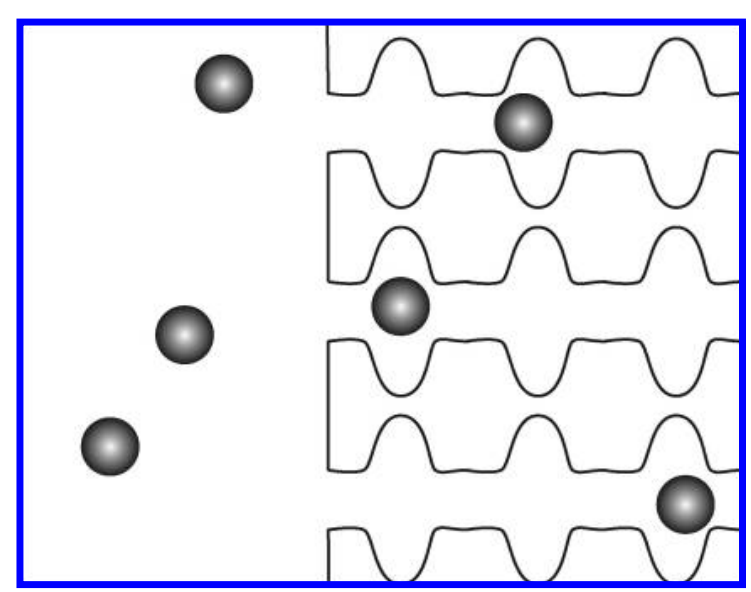

Figure 4. Schematic drawing of a zeolite in open contact with a reservoir; the molecules have to diffuse from the gas (or liquid) reservoir into the pores of the zeolite.

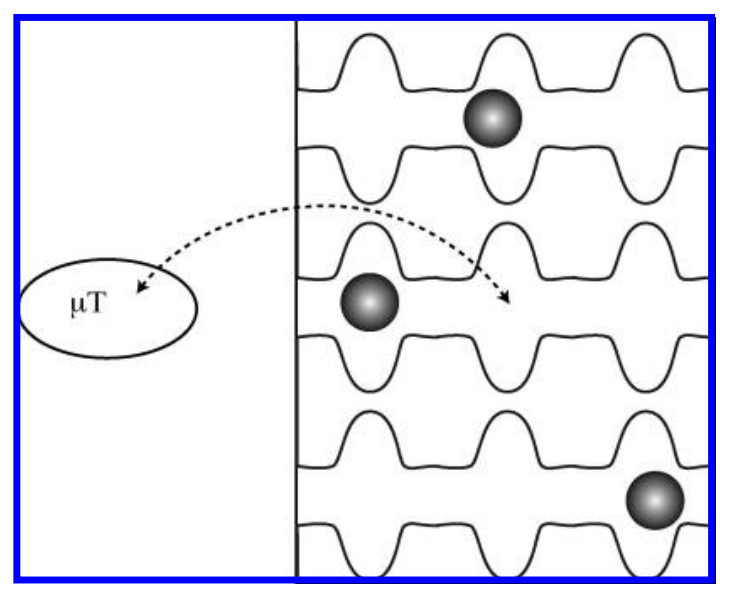

Figure 5. Grand-canonical ensemble; a zeolite in indirect contact with a reservoir that imposes the temperature, $T$, and chemical potential, $\mu$.

contains a gas or liquid (see Figure 4). The liquid or the gas is kept at a constant temperature and pressure or partial pressure in the case of a mixture. In equilibrium, the adsorbed gas molecules have the same temperature and chemical potential as the molecules in the container. The container can be seen as a reservoir that fixes the temperature and chemical potentials of the adsorbed components. In principle one could mimic the experimental setup by simulating a gas or liquid in contact with a reservoir. However, such a system is not very convenient from a simulation point of view, unless one is interested in the properties of this gas-zeolite interface.

The experimental setup setup shown in Figure 4 closely resembles the grand-canonical ensemble. In a grand-canonical ensemble, the temperature, volume, and chemical potentials are imposed. This ensemble can be sampled in a grand-canonical Monte Carlo simulation. Such a simulation is shown schematically in Figure 5. The difference with the experimental setup in Figure 4 is that, in a grand-canonical simulation, the reservoir and zeolite are not in direct physical contact, but the Monte Carlo procedure guarantees that the adsorbed molecules have an equal temperature and chemical potential in both the reservoir and in the zeolite. The input of the simulation is the temperature and chemical potential of the molecules in the reservoir, and the average number of adsorbed molecules is a result of the simulation. 
To compare a grand-canonical simulation with experimental data, one has to convert the imposed chemical potential (or fugacity) of each of the components of the reservoir to the corresponding partial pressures. At sufficiently low pressures, the reservoir can be considered as an ideal gas and one can simply assume that the fugacity is equal to the pressure. At higher pressures, however, one needs the equation of state of the fluid (mixture). One can use the experimental equation of state or one can use the equation of state of the model fluid that is being simulated. In practice, the experimental equation of state is know accurately for most of the systems of interest, which allows us to convert the experimental pressures to experimental fugacities. From a practical point of view, most authors prefer to present pressure versus loading and therefore assume that the experimental equation of state is a sufficiently accurate representation of the model fluid, to convert the imposed fugacity to (partial) pressure.

Of course, one can always carry out molecular simulation of the fluid without the zeolite to determine its equation of state. However, one needs a sufficiently accurate equation of state from the ideal gas density to the density of the fluid of the reservoir at the conditions of interest to make this conversion. This requires a large number of simulations. An alternative is to use the Gibbs-ensemble technique. ${ }^{20,21} \mathrm{In}$ this technique, one uses two separate simulation boxes: one for the reservoir and one for the zeolite. These boxes are coupled via Monte Carlo rules that allow for the exchange of particles. The acceptance rules ensure that the two systems are in equilibrium. The advantage of this simulation technique is that the pressure can be computed directly from the reservoir box and the loading directly from the zeolite box. Hence, with this simulation technique, one does not need to know the equation of state of the model fluid. ${ }^{22,23}$

The success of a Monte Carlo simulation relies on the efficiency at which one can generate a statistically independent configuration. For atoms, this can be achieved by giving a particle a random displacement. For molecules, however, one also has to perform Monte Carlo moves that change all internal degrees of freedom (for example, change the bond length, angle, torsion, etc.). In general, one has to design a separate scheme for every type of molecule to sample all configurations. This makes the Monte Carlo technique less suitable for a general purpose simulation package, and therefore most available Monte Carlo software is suitable for a particular subclass of molecules. Grand-canonical Monte Carlo works best if the acceptance of trial moves by which particles are added or removed is not too low. For atomic fluids, this condition effectively limits the maximum loading in a zeolite at which the method can be used. Although the grand-canonical Monte Carlo technique can be applied to simple models of nonspherical molecules, special techniques are required, since the method converges very poorly for all but the smallest polyatomic molecules.

\subsection{Simulating Free Energies}

In the previous sections, we have seen that Monte Carlo simulations can be used to compute the static properties while molecular dynamics properties give us both static as well as dynamic properties. However, both simulation techniques do not give us information on the free energy of the system. ${ }^{12}$ Special techniques are required to compute the free energy or chemical potential of a molecule. Details on these techniques can be found in the literature, ${ }^{12}$ here we focus

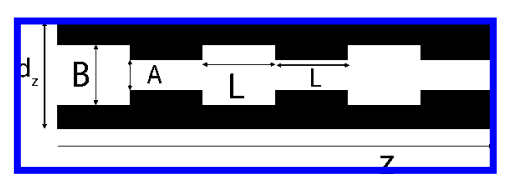

Figure 6. Model of a two-dimensional tubelike zeolite structure: $d_{z}$ is the pore diameter, $B$ is the diameter of the cage, $A$ is the diameter of the window separating the cages, and $L$ is the length of the cage, which is equal to the length of a window.

on those techniques that are often used to compute the energy of adsorbed molecules.

One of the more popular methods to compute the chemical potential is the Widom test particle method, ${ }^{24}$ in which the average energy of a ghost particle is calculated. A ghost particle is a particle that feels the same energy as a real particle, but the other particles in the system do not feel the presence of this particle. This energy $\left(\Delta^{+} U\right)$ is related to the excess chemical potential, ${ }^{12} \mu^{\text {ex }}$

$$
\exp \left(-\beta \mu^{\mathrm{ex}}\right)=\left\langle\int \mathrm{d} \mathbf{s}_{N+1} \exp \left[-\beta \Delta^{+} U\left(r_{N+1}\right)\right]\right\rangle_{N V T}
$$

where $s_{N+1}$ is the position of the ghost particle in reduced coordinates, so $\mathbf{s}=\mathbf{r} / L$, where $L$ is the box length, and the integration is over all possible positions of the ghost particle.

The chemical potential is of interest, since it is directly related to the Henry coefficient. In the limit of low external pressures, a good estimate of the adsorption isotherm can be obtained from the Henry coefficient $\left(K_{\mathrm{H}}\right)$. Under these conditions, the number of adsorbed molecules per unit volume $\left(\rho_{\mathrm{a}}\right)$ is proportional to the Henry coefficient and the external pressure $P$ :

$$
\rho_{\mathrm{a}}=K_{\mathrm{H}} P
$$

The Henry coefficient is directly related to the excess chemical potential of the adsorbed molecules

$$
K_{\mathrm{H}}=\frac{\rho_{\mathrm{a}}}{P}=\frac{1}{P} \frac{\beta P}{q(T)} q(T) \exp \left(-\beta \mu^{\mathrm{ex}}\right)=\beta \exp \left(-\beta \mu^{\mathrm{ex}}\right)
$$

where $q(T)$ is the kinetic part of the partition function. This equation shows that, by adding ghost particles to an empty zeolite, we can compute the excess chemical potential and the Henry coefficient. Another application of these free energy methods is to compute a free energy barrier.

Suppose we are interested in studying the diffusion coefficient of molecules adsorbed in the zeolite shown in Figure 3. If the molecules are large, the windows separating the cages form a barrier for the diffusion. By adding ghost particles to the system, we can compute the free energy as a function of the position in the zeolite and hence compute the free energy barrier the molecules have to cross if they hop from one cage to another.

Let us illustrate such free energy calculations with a "simulation" that can be done without a computer. We are interested to compute the Henry coefficients and free energy barriers in the zeolite shown in Figure 6. We assume that the adsorbed molecules do not interact with each other; they only interact with the zeolite in such a way that if a molecule is in the pores, either in the cage or in the window, the energy is zero (open part of the figure) and if the molecule is placed on top of a zeolite atom, the energy is infinite (black part of the figure). We can use eq 6 to compute the Henry coefficient of our system via the excess chemical potential by adding 


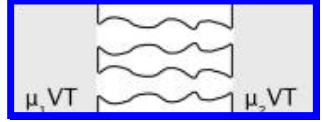

Figure 7. Dual Control Volume Grand Canonical Molecular Dynamics; two control volumes are coupled to a reservoir that imposes the temperature and the chemical potentials $\mu_{1}$ and $\mu_{2}$.

ghost particles at random positions in the zeolite. The ghost particle energy is

$$
\exp \left(-\beta \Delta^{+} U\right)= \begin{cases}0 & \text { if added in the black part } \\ 1 & \text { if added in the pore }\end{cases}
$$

The excess chemical potential is therefore simply related to the probability that a randomly inserted particle is inside the pore. This probability is equal to the fraction of the pore volume with respect to the total volume of the zeolite, which gives for the excess chemical potential for the system of Figure 6

$$
\beta \mu^{\mathrm{ex}}=-\ln \left(\frac{\pi A^{2} \times L+\pi B^{2} \times L}{\pi d_{z}^{2} \times 2 L}\right)=-\ln \left(\frac{A^{2}+B^{2}}{2 d_{z}^{2}}\right)
$$

We can also compute the excess chemical potential as a function of the position of the ghost particle in the zeolite. For example, we can divide our zeolite in small slabs and compute the excess chemical potential in each slab and assign the average value to the $z$-coordinate of the slab. For a single molecule the chemical potential is equal to the free energy, so we have for the free energy as a function of the position in the zeolite

$$
\beta F^{\mathrm{ex}}(z)=- \begin{cases}2 \ln \left(A / d_{z}\right) & \text { in the cage } 0<z<L \\ 2 \ln \left(B / d_{z}\right) & \text { in the window } L<z<2 L\end{cases}
$$

We see that if $B \ll A$, there is a diffusion barrier in our system. It is instructive to compute the minimum energy of a particle as a function of the position in the zeolite; since the energy is zero everywhere in the zeolite, we have

$$
U^{\min }(z)=0
$$

This illustrates that the diffusion barrier in our zeolite has a purely entropic region. In fact, if we were to use an energy minimization scheme, we would predict that there is no diffusion barrier in this system. This example therefore also demonstrates the important limitation of the energy minimization scheme.

\subsection{Dual Control Volume Grand Canonical Molecular Dynamics}

Heffelfinger and van $\mathrm{Swol}^{265}$ developed a combined molecular dynamics grand-canonical Monte Carlo technique which closely resembles the experimental situation in a membrane. The principles of the dual control volume grandcanonical Monte Carlo technique are illustrated in Figure 7. Two control volumes are introduced, and each is coupled to a reservoir that imposes the chemical potential and temperature. The system of interest is coupled to the control volumes via ordinary molecular dynamics. Suppose we give the two control volumes a different chemical potential; the system will respond by a steady flux of particles through the system. From these simulations one can compute the

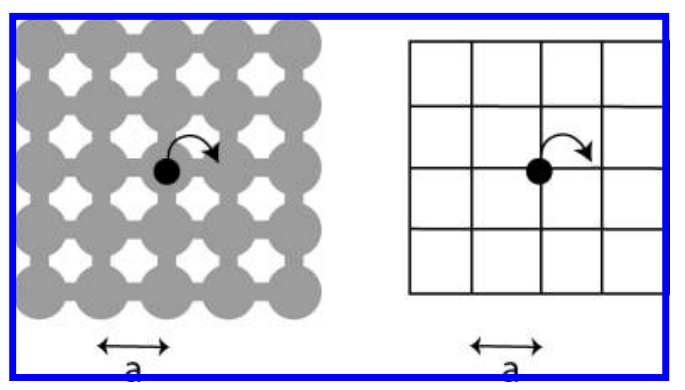

Figure 8. Model of a two-dimensional porous material consisting of cavities connected by narrow windows (left) and a coarse grained lattice model representing the porous material. The cages can occupy a single particle. All possible positions of a particle in a cavity are lumped into a single lattice site. The distance between two neighboring lattice sites, $a$, is equal to the distance between the middle of the cavities.

diffusion coefficient or study the effect of the surface, depending on the choice of the control volumes.

\subsection{Kinetic Monte Carlo}

The underlying theory of Monte Carlo simulation is that one generates a Markov chain of configurations with a desired limiting distribution, e.g., the Boltzmann distribution. In the equilibrium formulation, Monte Carlo time is rather a measure of the efficiency of the algorithm than a quantity that can be related to any physical time. For example, one can utilize a move in which one moves a particle to a randomly selected position; clearly such a move can be used even when diffusion barriers are very high, but such a move has no physical meaning, and as such Monte Carlo time cannot be related to real time if such moves are present.

In a kinetic Monte Carlo simulation, one mimics the dynamics of the underlying systems via a random selection of events. ${ }^{25}$ In this it is essential to make a link between Monte Carlo time and physical time. The best way to illustrate this is by an example.

Let us consider a two-dimensional porous material shown in Figure 8, which consists of cavities that are connected via a window and form a two-dimensional network. Let us assume that each cavity can accommodate a single molecule and that the narrow window forms a barrier for the diffusion. Because of these barriers the molecule will spent most of its time in a cavity and once in a while hop from one cavity to the next. This is identical to a random walk on a lattice. For a random walk on a lattice, it is well know that the distance a particle has "walked" on the lattice, if the particle makes $M$ independent jumps, is

$$
R^{2}=M a^{2}
$$

where $a$ is the lattice spacing. To introduce time in this equation, we assume that $M$ is the total number of jumps the particle makes in time $t$ :

$$
M=t r
$$

where $r$ is the hopping rate, i.e. the number of jumps per unit time. This gives the mean square displacement

$$
R^{2}(t)=r a^{2} t
$$

The mean square displacement of a particle in two dimensions can also be related to the diffusion coefficient $D$

$$
R^{2}(t)=2 D t
$$

Combining eqs 13 and 14 gives a relation between the diffusion coefficient and the hopping rate $r$ on the lattice. 
In the above discussion we have assumed that the particle "decides" to jump at fixed time intervals. However, a more realistic dynamics is obtained if we assume that a particle hops with time intervals that are distributed according to a Poisson distribution. Suppose that we carry out a molecular dynamics simulation and sample a small number of identical time intervals $\delta t$. We observe that, after $m$ of these intervals $(t=m \delta t)$, the average number of hops is $h_{\delta}$. The average hopping rate is then defined by

$$
r=\lim _{\delta t \rightarrow 0, t \rightarrow \infty} \frac{h_{\delta}}{t}
$$

In the limit $\delta t \rightarrow 0$, each time interval will contain, at most, one event. Of course, for a single particle, this seems a trivial observation, but if we would consider many particles, this limit ensures that this hopping process can be seen as a sequence of separate events, i.e., the time resolution is chosen such that two events cannot occur at the same time. ${ }^{25}$ As a consequence, each time interval has an equal probability, $r$ $\delta t$, for an event to occur. With these definitions, the probability that $n_{\mathrm{e}}$ events occur in a time $t$ is given by the Poisson distribution, which reads in the limit $\delta t \rightarrow 0$

$$
P\left(n_{\mathrm{e}}\right)=\frac{r t^{n_{\mathrm{e}}}}{n_{\mathrm{e}} !} \exp [-r t]
$$

where the expected number of events in a time $t$ is given by $\left\langle N_{\mathrm{e}}\right\rangle=r t$. We are interested in the distribution of the time intervals between two events, which follows from this equation by setting $n_{\mathrm{e}}=1$. Hence, the time step between two events, $\Delta t_{\mathrm{h}}$, follows from

$$
\Delta t_{\mathrm{h}}=\frac{-\ln [\operatorname{Ran}]}{r}
$$

where Ran is a random number between 0 and 1 . Since the hopping process can now be seen as a chronological sequence of distinct events, we could also perform a kinetic Monte Carlo simulation to compute the mean square displacement of a particle. If we have a single particle and assume that at every Monte Carlo step we make a random jump to one of the neighboring lattice sites, the link to physical time is made by specifying the hopping rate. The input of a kinetic Monte Carlo simulation is therefore the hopping rate and the underlying lattice.

Kinetic Monte Carlo is therefore not a molecular simulation technique but operates on the mesoscopic level and can therefore access longer time and length scales. For the present example, the solution could be obtained analytically and kinetic Monte Carlo simulations are not really needed. For more complex problems, higher loadings, mixtures, etc., simulations are essential. Several groups have applied kinetic Monte Carlos simulations to zeolites. ${ }^{26-29}$ For a detailed description of the kinetic Monte Carlo technique and its application to zeolites, we refer to the excellent review of Auerbach. $^{30}$

\subsection{Ab Initio Molecular Dynamics}

In conventional classical simulation techniques (MD or MC), we assume that the intermolecular potentials are known and given in a closed form. From a fundamental point of view, this approach has important limitations, since it assumes, for example, that the molecules do not react. In fact, the intermolecular interactions are an approximation of the solution of the Schrödinger equation. In an ab initio molecular dynamics simulation, one solves the Schrödinger equation every time step and a molecular dynamics simulation is performed without any prior assumption of the intermolecular potentials. Of course, these electronic structure calculations are very expensive calculations, and therefore the number of time steps or configurations that can be computed is far less. For example, typical ab initio molecular dynamics simulations are on the order of $1-10 \mathrm{ps}$, whereas in a conventional molecular dynamics simulation 10-100 ns can be obtained.

Most of the early computational studies using ab initio molecular dynamics focus on the structure of the zeolites, ${ }^{31}$ while later studies discuss the reactivity ${ }^{32,33}$ or adsorption. ${ }^{34-38}$ A very important recent application deals with the formation of zeolites ${ }^{39,40}$

\subsection{Hybrid Techniques: The QM/MM Approach}

The use of ab initio molecular dynamics (see section 2.7) is a very expensive technique, since it is using a full quantum chemical description of the entire zeolite. Chemical reactions in a zeolite often occur in a small part of the zeolite; it would therefore be much more attractive to use a quantum chemical description of that part of the zeolite that is participating in the chemical reaction, while the remainder of the zeolite is treated using the classical simulation techniques. ${ }^{41,42}$ From a simulation point of view, an important question is how to make the transition from the quantum to the classical part. Clearly, the result should not depend on the arbitrary division of the system into a classical part and a quantum part.

These methods have been applied by Sauer and co-work$\mathrm{ers}^{43-45}$ to study, for example, the complete reaction path of a skeleton isomerization in the pores of a zeolite ${ }^{45}$ or by Deka et al. ${ }^{46}$ to study the preferred locations of Ti centers in MFI.

\section{Advanced Simulation Techniques}

In the previous section we have given a short introduction of the standard simulation techniques that are commonly used in the literature. The next step would be a reliable intermolecular potential that describes the interactions between the molecules sufficiently accurately that the thermodynamic and transport data that are computed give a reliable estimate of the experimental system. The efficiencies of these standard simulation techniques, however, are often limiting these simulations to noble gases or small molecules. These simulations give important insights into the general aspects of adsorption and diffusion, but for petrochemical applications one would like to understand the behavior of the molecules of interest at catalytic conditions. Interestingly, novel simulation techniques have been developed in the past decade that make these types of simulations possible. In this section, these simulation techniques will be discussed. The discussion will focus on the general principles and ideas behind these techniques, for a more detailed discussion on the technical details, the reader is referred to the literature.

\subsection{Configurational-Bias Monte Carlo}

Both molecular dynamics and the Monte Carlo technique are very efficient for atoms or small molecules, but for large molecules both methods require significant amounts of CPUtime. For example, June et al. ${ }^{47}$ studied the relaxation of 
butane and hexane in MFI using molecular dynamics and concluded that the zeolite slowed down the relaxation of these molecules several orders of magnitude; the longer the chain length, the slower the relaxation. Hence, the CPUrequirements increase significantly for MD simulations of these long chain alkanes. The diffusion coefficient of linear alkanes in MFI is sufficiently high that these can be simulated using MD, ${ }^{48}$ but for the monobranched alkanes, MD can only be used at very high temperatures. ${ }^{18,49}$ Branched alkanes in MFI preferentially adsorb in the intersections between the zigzag and straight channels; ${ }^{50}$ the diffusion is therefore an activated process in which the molecule jumps from one intersection to another. ${ }^{51}$ This very slow diffusion path could be avoided via a Monte Carlo simulation in which we generate a new configuration at a random position in the zeolite. Equation 3 shows that the probability that such a move will be accepted depends on the energy difference between the new and the old configurations. Clearly, if we generate a new position on top of a zeolite atom, the attempt will be rejected. For a chain molecule this implies that none of the atoms should overlap with the zeolite atoms. If for methane this probability is 1 out of 1000 attempts, for ethane this will be of the order 1 out of $10^{6}$, and for octane 1 out of $10^{24}$. The conventional Monte Carlo method is therefore very efficient for noble gases or small molecules, but for the long chain alkanes it is equally inefficient as molecular dynamics.

The configurational-bias Monte Carlo (CBMC) technique has been developed to make the insertion of long chain molecules in moderately dense liquids possible. The original configurational-bias Monte Carlo technique has been developed for lattice models ${ }^{52,53}$ and has been extended to continuous models. ${ }^{54}$ Here, we show how this method can be used to simulate the adsorption of long chain hydrocarbons in zeolites. In a CBMC simulation the molecules are not inserted at random but grown atom by atom. This growing process introduces a bias which can be removed exactly by adjusting the acceptance rule. ${ }^{12}$

To generate a new configuration of a molecule, we use the following steps based on the method developed by Rosenbluth and Rosenbluth. ${ }^{55}$

1. For the first atom, a random position in the zeolite is generated, and the energy of an atom at this position is calculated. This energy is denoted by $u_{1}$.

2 . For the following atoms, say atom $i$, a set of $k$ trial positions is generated; see Figure 9. We denote these positions by $b=\left(b_{1}, b_{2}, \ldots, b_{k}\right)$. For each of these trial positions, the energy is calculated with the atoms of the other molecules (of the zeolite and of other alkanes) and with those atoms of the molecule that are already grown. This energy is denoted by $u_{i}\left(b_{j}\right)$.

3. Out of these $k$ trial positions, one is selected, say $j$, with a probability

$$
p_{i}\left(\mathbf{b}_{j}\right) \mathrm{d} \mathbf{b}_{j}=\frac{\exp \left[-\beta u_{i}\left(\mathbf{b}_{j}\right)\right] d \mathbf{b}_{j}}{\mathrm{w}(i)}
$$

in which

$$
\mathrm{w}(i)=\frac{1}{k} \sum_{j=1}^{k} \exp \left[-\beta u_{i}\left(\mathbf{b}_{j}\right)\right]
$$

Equation 15 ensures that those conformations with the lowest energy have the highest probability of being selected.

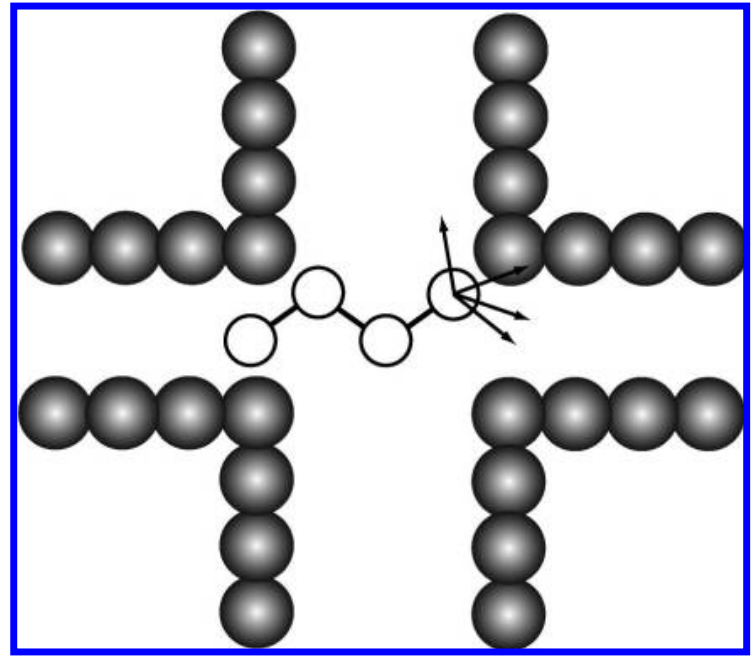

Figure 9. Configurational-bias Monte Carlo (CBMC) scheme, in which a chain is grown atom by atom in the pores of a zeolite; the arrows represent the $k$ trial orientations.

4. After repeating step 2 until the entire alkane of length $M$ has been grown, we compute the Rosenbluth factor of the new configuration

$$
W(n)=\exp \left(-\beta u_{1}\right) \prod_{i=2}^{M} \mathrm{w}(i)
$$

We use a similar algorithm to compute the Rosenbluth factor of the old conformation.

1. A molecule is selected at random; the energy of the first atom is calculated and is denoted by $u_{1}$.

2. For the following atoms, the external energy $u_{i}$ is calculated and a set of $k-1$ trial orientations is generated. Using this set of orientations and the actual position of atom $l, b_{1}$, we calculate

$$
\mathrm{w}(l)=\frac{1}{k}\left\{\exp \left[-\beta u_{i}\left(\mathbf{b}_{1}\right)\right]+\sum_{j=2}^{k} \exp \left[-\beta u_{i}\left(b_{j}\right)\right]\right\}
$$

3. After repeating step 2 until all $M$ atoms of the alkane have been considered, we calculate for the entire molecule the Rosenbluth factor of the old conformation

$$
W(o)=\exp \left(-\beta u_{1}\right) \prod_{i=2}^{M} \mathrm{w}^{\mathrm{ext}}(i)
$$

We can use these algorithms to generate a new conformation and compute the Rosenbluth factors of the old and new configurations to perform $N V T$ simulations, grand-canonical calculations, and free energy calculations. In the $N V T$ and grand-canonical simulations, it is important that the bias, which is introduced by the selection of the most favorable conformation, is removed by appropriate acceptance rules. For example, for an $N V T$ simulation, one can use the CBMC scheme to remove a randomly selected molecule and regrow it at a random position in the zeolite; this move is not accepted or rejected on the basis of the energy difference (compare eq 3) but on the basis of the ratio of the Rosenbuth weights: ${ }^{12}$

$$
\operatorname{acc}(o \rightarrow n)=\min (1, W(n) / W(o))
$$

The complete proof that this acceptance rule removes the bias exactly can be found in the literature. ${ }^{12}$ Similar acceptance rules can derived for the grand-canonical ensemble. $^{12}$ 
We can also use the CBMC scheme to compute the free energy of an alkane in a zeolite. This application is similar to the test particle (see section 2.4) insertion method. The CBMC scheme is used to generate a conformation of a molecule, the test particle, and to compute its Rosenbluth factor $W$. The excess chemical is directly related to the ensemble average of the Rosenbluth factor

$$
\beta \mu^{\mathrm{ex}}=-\ln \langle W\rangle
$$

The basic CBMC scheme has been extended to branched molecules ${ }^{50,56,57}$ and cyclic molecules. ${ }^{58-61}$ The scheme that is discussed here is appropriate for united atom models; for all atom models a slightly different scheme should be used. ${ }^{62,63}$ Several tricks have been invented to increase the efficiency of a CBMC simulation. ${ }^{64}$

Maginn et al ${ }^{65}$ have shown that, in the limit of very low loading, the partition function of an adsorbed chain molecule can be computed numerically using a scheme similar to CBMC. Using this configurational-bias Monte Carlo Integration scheme, the heat of adsorption and Henry coefficients of the $n$-alkanes in silicalite- 1 have been computed.

\subsection{Rare Event Simulations}

Diffusion coefficients of adsorbed molecules can vary as much as 10 orders of magnitude. If the diffusion coefficient is sufficiently high, straightforward MD can be used to simulate the system. If, however, the diffusion coefficient is very low, molecules are trapped in low (free) energy sites and only occasionally hop from one adsorption site to another. To compute a diffusion coefficient reliably, one has to observe a sufficient number of hops. Most of the CPUtime is spent on molecules that "wait" close to an adsorption site until a fluctuation gives them sufficient kinetic energy to take the barrier between adsorption sites. The higher the barrier, the longer the molecules remain trapped, and on the time scale of a MD simulation, such a hopping becomes a very rare event.

Special techniques have been developed to simulate such rare events. ${ }^{12}$ The basic idea is to compute the hopping rate in two steps. ${ }^{66,67}$ First we compute the probability that a molecule can be found on top of the barrier followed by a separate simulation in which the average time is computed it takes one molecule on top of the barrier to actually cross it.

Let us consider as an example the system shown in Figure 3 ; the adsorption sites are in the cavities, and the windows are the diffusion barriers. In a rare event simulation, it is important to define an appropriate reaction coordinate which characterizes the progress of the "reaction". In the case of a single atom, an obvious choice is the position along the tube, and a typical free energy as a function of the reaction coordinate is shown in Figure 10. The probability to find a molecule on top of the barrier can be computed directly from the free energy profile

$$
P\left(q^{*}\right) \mathrm{d} q=\frac{\exp \left[-\beta F\left(q^{*}\right)\right] \mathrm{d} q}{\int_{-\infty} \mathrm{d} q \exp [-\beta F(q)]}
$$

where $q^{*}$ is defined as the top of the barrier. $F(q)$ is the free energy as a function of the order parameter. This free energy can be computed using the techniques described in sections 2.4 and 3.1 .

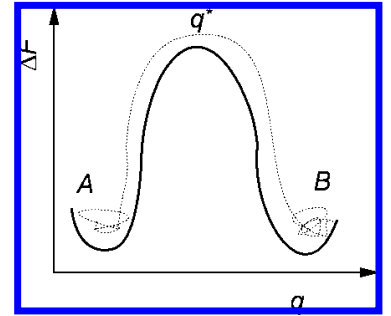

Figure 10. Free energy as a function of the reaction coordinate $q$; $\mathrm{A}$ and $\mathrm{B}$ correspond to the cages of the zeolite, and $q^{*}$ corresponds to the window between the cages, which is the bottleneck for the diffusion.

The second step involves the average time it takes a molecule to cross the barrier. The simplest approach is to assume that transition state theory (TST) holds. A molecule that arrives at the top of the barrier is assumed to be in equilibrium with its surrounding; as a consequence, the velocity distribution is given by the Maxwell distribution corresponding to the temperature of the system. TST assumes that half of the molecules that reach the barrier also cross the barrier, i.e., those with a positive velocity of the order parameter. The TST approximation of the hopping rate is

$$
k^{\mathrm{TST}}=\frac{1}{2}|\dot{q}| P\left(q^{*}\right)=\sqrt{\frac{k_{\mathrm{B}} T}{2 \pi m}} \frac{\exp \left[-\beta F\left(q^{*}\right)\right]}{\int_{-\infty}^{q *} \mathrm{~d} q \exp [-\beta F(q)]}
$$

The advantage of TST is that one only has to compute the free energy as a function of the reaction coordinate to compute the hopping rate. The disadvantage is that one does not know in advance whether the assumptions underlying TST hold. In addition, TST also assumes that the transition state is known exactly; that is, the top of the free energy, $q^{*}$, exactly corresponds to the true transition state. In practice, we do not know the free energy exactly, and we therefore can only approximate the transition state.

In the system we consider in Figure 3, the choice of the reaction coordinate is straightforward. However, in practice one has to be very careful. Consider, for example, the zeolites shown in Figure 11. Both zeolites are one-dimensional channels of cages connected via narrow windows. In analogy with the system of Figure 3, one would take as order parameter the position of the atom projected on the axis of the channel (red short dashed line), but we could have also taken a projection on a line through the window that has an angle with the channel axes (blue long dashed line). Depending on the particular choice, the free energy of the transition state will be different. If we use these free energies and compute the hopping rate using eq 23 , we would find different values of this hopping rate. In fact, TST state theory gives an upper limit; the true hopping rate is always lower compared to the TST result. It is therefore important to select the reaction coordinate that gives the highest free energy of the transition state. Since the free energy appears in the exponential in eq 23, TST theory can give a large error in the hopping rate if the choice of reaction coordinate is far from optimal. It may look strange to use a reaction coordinate that does not correspond to the direction of diffusion. Figure 11 shows that for the zeolite in which the window is not perpendicular to the channel axis, this choice results in a higher free energy of the transition state. This example illustrates that even for the diffusion of an atom, the choice of reaction coordinate can be nontrivial. For molecules, the 


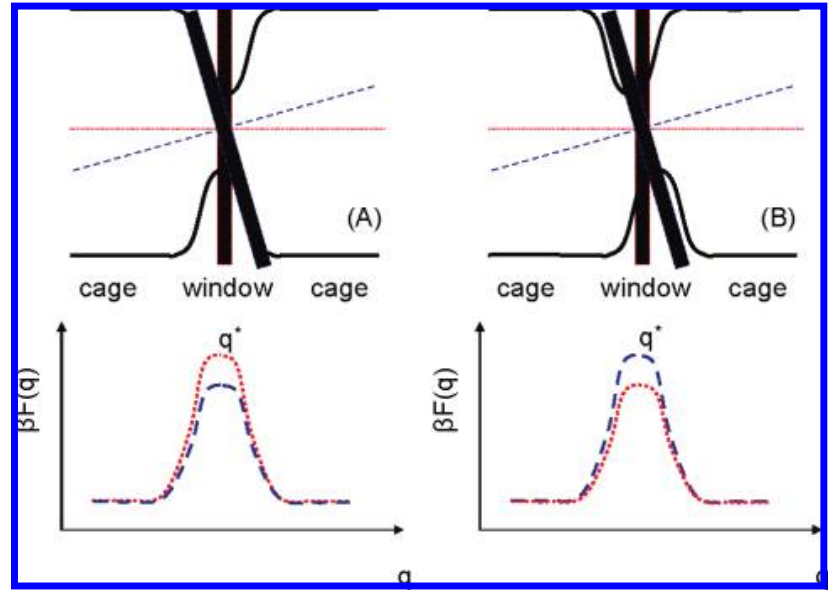

Figure 11. Comparison of reaction coordinate; two different choices of the reaction coordinate $q$. The red (short dashed) choice is parallel to the axis of the zeolite, while the blue choice (long dashed) makes an angle with the axis. In the left half of the figure, the window is perpendicular to the axis of the zeolite, while in the right half of the figure, the window takes an angle. The shaded areas show the part of the zeolite for which the free energy is projected on the transition state $q^{*}$. The bottom figures show that, depending on the choice of reaction coordinate, the free energy of the transition state, $F\left(q^{*}\right)$, has a different value.

number of possible reaction coordinates increases dramatically and it will be impossible to compute the free energies for all possible reaction coordinates.

Finally, even if one is able to select the optimal reaction coordinate, TST may not give the correct hopping rate, since in eq 23 it is assumed that all particles that start in one cage and arrive on top of the barrier with a positive velocity of the order parameter arrive in the "product" cage. TST ignores the possibility that such a particle recrosses the barrier and returns in the cage in which it originates due to, for example, collisions with the zeolite atoms.

To compute the true hopping rate, one has to correct the TST to take into account the recrossing of the barrier. These recrossings can be intrinsic to the system or due to the nonoptimum choice of reaction coordinate. This correction is obtained using, for example, the Bennet-Chandler ${ }^{66,67}$ approach in which molecular dynamics simulations are performed to compute the transmission coefficient, $\kappa$. The computation of the transmission coefficient involves many MD simulations which all start on top of the barrier and for which we determine the fraction that ends up in the product cage. The time dependent transmission coefficient is defined as

$$
\kappa(t)=\frac{k(t)}{k^{\mathrm{TST}}}=\frac{\left\langle\dot{q}(0) \delta\left(q(0)-q^{*}\right) \theta\left(q(t)-q^{*}\right)\right\rangle}{0.5\langle|\dot{q}(0)|\rangle}
$$

where $\theta(q)$ is the Heaviside function $(\theta(q)=1$ if $q>0$ and $\theta(q)=0$ otherwise) and $\delta(q)$ is the Dirac delta function. The delta function in eq 24 indicates that the trajectories are initiated on top of the barrier, and the Heaviside function takes a value if the particle is on the product side of the barrier. Equation 24 shows that if all particles with a positive velocity of the order parameter stay in the product cage, the transmission coefficient is one, and transition state theory gives the exact result. For those systems in which barrier recrossings are important, eq 24 gives a plateau value for intermediate times that can be used to correct the TST hopping rate. The important aspect of these MD simulations is that they are initiated on top of the barrier, which is a very unfavorable configuration for which the relaxation to equilibrium, one of the cages, is relatively fast, and these simulations therefore do not require much CPU-time for most systems.

In this section we have focused on one-dimensional order parameters, and for some systems it can be desirable to use a multidimensional order parameter. TST can generalized to higher dimensions, and one has to locate the saddle point in such a multidimensional space for which special techniques have been developed. ${ }^{68}$ For some systems, however, the dynamics on top of the barrier can be diffusive; because of collisions with the atoms of the zeolite, a particle may spend a relatively long time on top of the barrier before it falls into one of the cages. For such systems it can be advantageous to compute the hopping rate using the approach of Ruiz-Montero et al. ${ }^{69}$ In the methods we have discussed so far we assume that a good estimate of the transition state can be obtained. Although eq (24) can be used to correct an unfortunate choice or reaction coordinate; if the transmission coefficient is very small, it is expensive to compute it accurately. Transition path sampling ${ }^{70-72}$ has been developed to compute hopping rates without prior knowledge of the reaction coordinate. This method can also be used to check whether the assumed transition state resembles the true transition state.

\section{Molecular Models: Inter- and Intramolecular Potentials}

The most important inputs of molecular simulation are the inter- and intramolecular potentials describing the interaction between the zeolite atoms and the atoms of the adsorbate. It is essential that these potentials provide an accurate representation of the interactions of the experimental system that is being simulated. Ideally, one would like to use the best quantum chemical approach to compute the interactions of the atoms every time step. Unfortunately, the CPU requirement of such an approach is often beyond reach, and therefore most molecular simulation involves approximate potentials that have been (partially) fitted to experimental data. In this section we discuss some of the approaches used in the literature.

\subsection{The Zeolite Structure}

The starting point is the crystal structure of the zeolite. For most zeolites the crystal structure has been resolved and this information can be found in a convenient format on the Internet. ${ }^{73}$ This information allows us to generate the atomic coordinates of an arbitrary number of units cells of a zeolite crystal.

Most simulation studies follow the assumptions pioneered by Kiselev and co-workers ${ }^{74}$ for the adsorption of nonpolar molecules in which the zeolite is assumed to be rigid. If a rigid lattice is assumed, one does not need a model for the zeolite-zeolite interactions. In addition, if one would like to study, say, the adsorption at very low concentration of adsorbed molecules, including the flexibility of the zeolite, this increases the CPU-time by 2 orders of magnitude.

Several force fields have been developed to take into account the flexibility of the zeolite lattice. The accuracy of these models can partly be assessed via a comparison of the calculated vibrational IR spectra with the experimental ones. 
To limit the CPU requirements of a fully flexible zeolite, models have been developed in which the normal vibrational modes and harmonic crystal approximation are used. ${ }^{75}$ Since these models have been discussed in detail by Demontis and Suffritti, ${ }^{76}$ we refer to this review for details on these models and further references. In the subsequent sections, we will focus on the influence of this approximation on the computed properties.

\subsection{Adsorbate-Adsorbate Interactions}

For the simulation of hydrocarbons, various models have been proposed in the literature. The most realistic models are all-atom models in which both the carbon and hydrogen models are considered explicitly. In united-atom models, the $\mathrm{CH}_{3}, \mathrm{CH}_{2}$, or $\mathrm{CH}$ groups are considered as single atoms. The united-atom models give quite an accurate description of hydrocarbons in the liquid state, but it has been argued that these models fail to give a correct description of the phases of solid hydrocarbons. ${ }^{77}$ Only at very high pressures or low temperatures could one envision solidlike behavior of hydrocarbons adsorbed in zeolites, suggesting that unitedatom models should give reasonable descriptions. From a computational point of view, the united-atom models are more efficient and have less parameters that have to be determined. Therefore, most studies use a united-atom approach and comparison with experimental data for the adsorption and diffusion shows a satisfactory description of the experimental data.

For the adsorbate-adsorbate interactions, it is convenient to distinguish the intramolecular interactions and the intermolecular interactions. The intramolecular interactions are very important to arrive at a realistic representation of the conformation of the adsorbate molecules. Fortunately, these potentials can be based on quantum chemical or spectroscopic data, and therefore for most molecules these models give a sufficiently accurate description of these intramolecular interactions. In addition, comparisons of various models for, for example, the torsion or bond-bending show little influence on the thermodynamic properties, such as the vapor-liquid curve. $^{78-80}$

Most of the commonly used force fields have been developed for biological applications and give a very accurate description of, for example, the density of a hydrocarbon at ambient conditions but fail to give a correct description at the critical point. ${ }^{79}$ Whereas most adsorption and diffusion studies in zeolites are performed at ambient conditions, in practical applications one often considers much higher temperatures. It is therefore important to ensure that the hydrocarbon force fields give a reasonable description at a wide range of temperatures and pressures. For the hydrocarbons, special force fields, both for united-atom and allatom models, have been developed that give an accurate description of the vapor-liquid coexistence curve over a wide range of conditions. ${ }^{57,78,80-86}$ Figure 12 gives a typical illustration of the accuracy that can be obtained with these types of models.

\subsection{Zeolite-Adsorbate Interactions}

In most simulation studies the zeolite-adsorbate interaction parameters are obtained from some kind of fitting to experimental data (for some exceptions, see refs 87-89). However, the uncertainties in the models and in the experimental data do not allow for one to obtain a unique

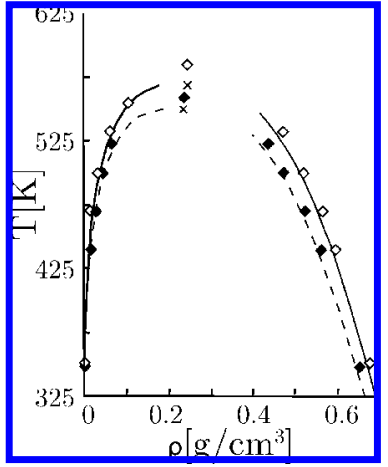

Figure 12. A typical illustration of the reliability of an optimized united-atom model in predicting the vapor-liquid coexistence curves for a branched hydrocarbon. The figure shows the results of the TraPPE-UA force field, as has been developed by Siepmann and co-workers, for 2,5-dimethylhexane and 3,4-dimethylhexane. Experimental coexistence data and critical points are shown as long dashed lines (2,5-dimethylhexane), solid lines (3,4-dimethylhexane), and times signs. Simulated coexistence densities and extrapolated critical points for the force field are shown as filled diamonds (2,5dimethylhexane) and open diamonds (3,4-dimethylhexane). (Figure is based on the data from ref 57).

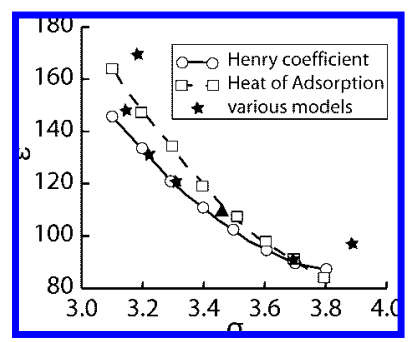

Figure 13. Optimum Lennard-Jones parameters for the heat of adsorption (dashed line with squares) and Henry coefficient (solid line with circles) of methane in silicalite. ${ }^{90}$ The crossing of the lines gives the parameters for which both the Henry coefficient and heat of adsorption agree with the experimental results. For comparison, the stars are the parameters of the model proposed by Bezus et al., ${ }^{74}$ Goodbody et al., ${ }^{91}$ Hufton et al., ${ }^{92}$ and Demontis et al. ${ }^{93,74,91-93}$ The triangle gives the model of Dubbeldam et al. ${ }^{94}$

set of parameters. For example, the parameters obtained from fitting to diffusion coefficients can be different from those fitted to, for example, heats of adsorption or Henry coefficients.

To illustrate this, Figure 13 compares the different sets of parameters for methane adsorbed in silicalite (MFI) that have been published in the literature, ${ }^{74,90,91,93,94}$ in which methane is described as a united atom. Such a model has exactly two parameters, with the $\varepsilon$ and $\sigma$ of the Lennard-Jones interactions describing the dispersive interactions with the oxygen of the zeolite. This figure illustrates that for any reasonable value of $\sigma$ one can find a corresponding value of $\varepsilon$ to reproduce the experimental heat of adsorption. Hence, to fix these parameters, one needs an additional experimental value of, say, the Henry coefficient. Given the experimental uncertainties in the Henry coefficients, one may end up with different sets of parameters that all give a reasonable description, depending on which experimental data set one uses.

The difficulties in obtaining reliable data have been emphasized by Dubbeldam et al. $;{ }^{94}$ an illustration of this point is given in Figure 14; although the experimental data are at relatively low pressures, a comparison with the results of molecular simulations shows that the experiments are not in the Henry regime. 


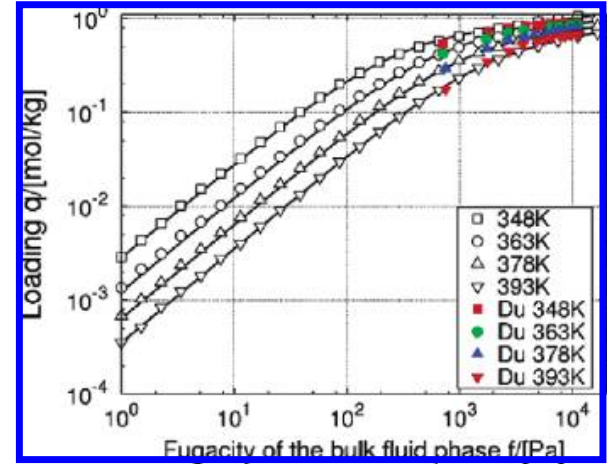

Figure 14. Isotherm of $n$-hexane in MWW at various temperatures; the lines are the simulation results, and the closed symbols are experimental data. Reprinted with permission from ref 94. Copyright 2004 American Chemical Society.

Pascual and co-workers ${ }^{95,96}$ used an approach that relies less on the availability of accurate experimental data. This method relies on the assumption that alkane-zeolite interactions can be estimated from the Lorentz-Berthelot mixing rules for the anisotropic united-atom model:

$$
\varepsilon_{i-j}=\sqrt{\varepsilon_{i} \varepsilon_{j}} \quad \text { and } \quad \sigma_{i-j}=0.5\left(\sigma_{i}+\sigma_{j}\right)
$$

and as the alkane-alkane interactions have been obtained from the vapor-liquid curve, there is only the need to determine the $\varepsilon_{\mathrm{O}}$ and $\sigma_{\mathrm{O}}$ associated with the oxygen-oxygen interactions of the zeolite to fix all other parameters. As the experimental isotherms of butane in silicalite are known very accurately, Pascual et al. selected four experimental points at low and high pressures of two different isotherms and used a minimization procedure to optimize the $\varepsilon_{\mathrm{O}}$ and $\sigma_{\mathrm{O}}$. With these parameters, the model of Pascual and co-workers gave a very good prediction of the adsorption isotherms of other linear and branched hydrocarbons in $\mathrm{MFI}^{95}$ and $\mathrm{FER}^{96}$ and of alkane-alkene mixtures in MFI. ${ }^{96}$

A very different strategy was introduced by Dubbeldam and co-workers. ${ }^{94,97}$ The basis of their fitting procedure was the observations that steps and kinks in the adsorption isotherms are directly related to the structure of the zeolite and are very sensitive to the parameters of the model. For example, in MFI, there are four intersections of the zigzag and straight channels per unit cell (see Figure 37). Branched hydrocarbons preferentially adsorb at these intersections and only at sufficiently high pressure between the intersections. This adsorption behavior causes a plateau at the adsorption isotherm which is exactly at four molecules per unit cell, as is illustrated in Figure 15. In addition, Dubbeldam et al. show that the shape of the adsorption isotherms is very sensitive to small changes in the energy and size parameter of the Lennard-Jones potential. Figure 15a shows the computed adsorption isotherms for a size parameter that is too small; hence, the effective size of the molecules is too small and the intersections are not the preferential adsorption sites and no step is observed. In contrast, Figure 15b shows a case in which the size parameter is too large. For this parameter, the adsorption isotherm does have a plateau, but irrespective of the energy parameter, the plateau is much too large. The important observation of Dubbeldam et al. is that the difference between a size parameter that is much too small $\left(\sigma_{\mathrm{O}-\mathrm{CH}}=3.36 \AA\right)$ and one that is much too big $\left(\sigma_{\mathrm{O}-\mathrm{CH}}=\right.$ $3.60 \AA$ ) is surprisingly small. Dubbeldam et al. showed that this procedure uniquely determined the $\varepsilon_{\mathrm{CH}_{x}-\mathrm{O}}$ and $\sigma_{\mathrm{CH}_{x}-\mathrm{O}}$ with a precision better than $5 \mathrm{~K}$ and $0.02 \AA$, respectively.

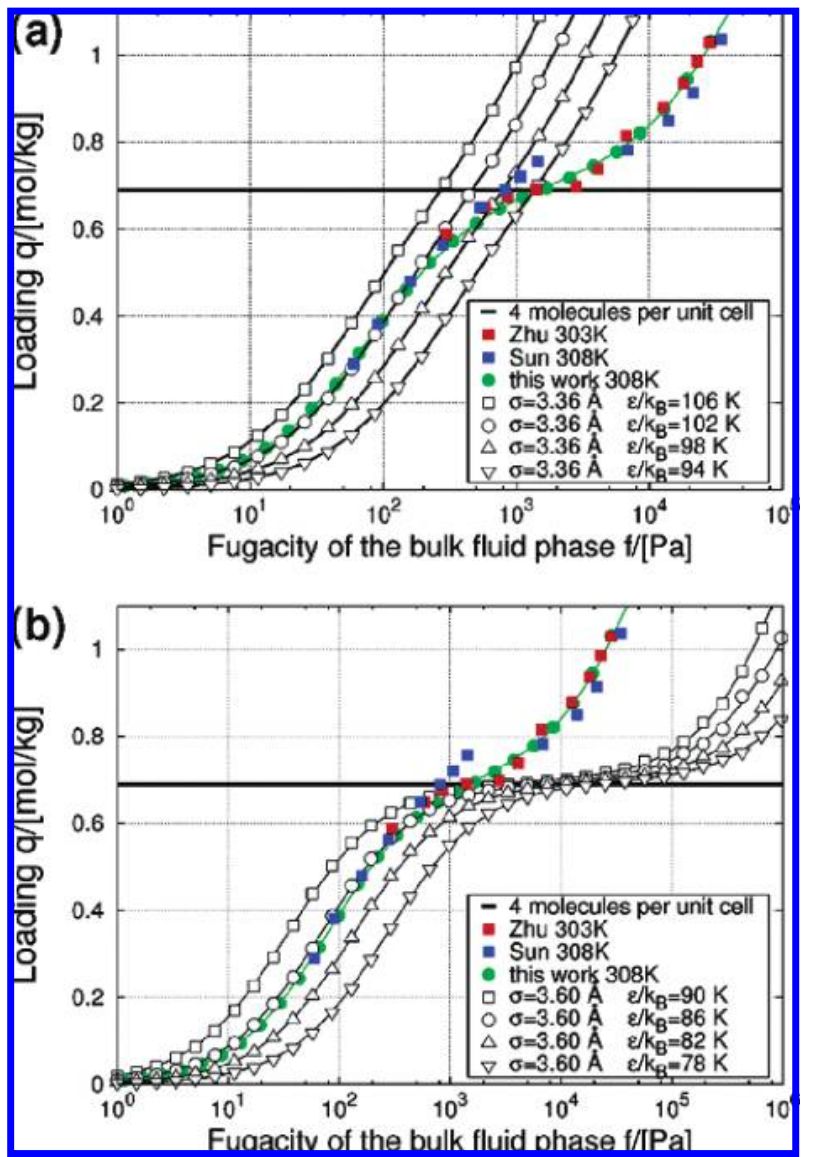

Figure 15. Illustration of the sensitivity of the adsorption isotherms with respect to small changes of the parameters of the LennardJones potential $\varepsilon$ and $\sigma$ for 2-methylpropane at $308 \mathrm{~K}$ in MFI. Part a gives the case for which the size parameter is too small $\left(\sigma_{\mathrm{O}_{-} \mathrm{CH}_{3}}\right.$ $=3.36 \AA$ ) irrespective of the choice of the energy, while part $\mathrm{b}$ gives the case for a too high value $\left(\sigma_{\mathrm{O}_{-} \mathrm{CH}_{3}}=3.60 \AA\right)$. Reprinted with permission from ref 94. Copyright 2004 American Chemical Society.

An extension of this approach to alkenes has been made by Jakobtorweihen et al. ${ }^{98}$ and Liu et al. ${ }^{99}$

It is instructive to compare the approached of Pascual and co-workers with the one of Dubbeldam et al. The method Pascual has as important advantage that the parameters are transferable; once these values have been fixed, they, in theory, can be transferred to any other hydrocarbon group, e.g. alkenes. ${ }^{96}$ In the approach of Dubbeldam et al., one has to redo the fitting procedure for the alkenes, and this requires accurate experimental data, which may not always be available. On the other hand, the approach of Dubbeldam et al. may give a better description. For example, the experimental data for 2-methylpropane in MFI show a clear step in the adsorption isotherm, which is correctly described with the model of Dubbeldam et al. but not with the model of Pascual et al. At this point it is important to note that it is very encouraging to see that one has to go into such detail to make a distinction between these two approaches, as the agreement with experimental data is for both approaches remarkably good. Attempts have been made to obtain force fields from fitting to ab initio calculations, ${ }^{100}$ but the size of the quantum calculations made it unavoidable to introduce some empirical corrections to the obtained parameters. 


\subsection{Cation-Zeolite-Adsorbate Interactions}

Most simulation studies use zeolites consisting of only silicon and oxygen. In practice, however, the silicon in zeolites can be substituted with other metal atoms, such as aluminum, boron, iron, or germanium. Depending on the structure, these changes can have a profound influence on the catalytic or adsorption properties of the zeolite.

\subsubsection{Cation-Zeolite Interactions}

Most research focuses on those structures that contain aluminum substitutions. Since the partial charges of silicon and aluminum are $4+$ and $3+$, respectively, replacing silicon by aluminum introduces a negative charge in the zeolite framework. This charge has to be compensated by either protons or larger cations. During the synthesis, normally $\mathrm{Na}^{+}$ ions are chosen as counterions, but after the synthesis, they can be replaced by several other cations, including $\mathrm{Cs}^{+}, \mathrm{Li}^{+}$, and $\mathrm{Ca}^{2+}$, by way of ion exchange. For catalytic applications, exchange with $\mathrm{H}^{+}$is very important, as it introduces the acid sites that are responsible for the catalytic activity. The type and location of cations can influence the adsorption properties significantly. It is therefore important to know the locations of the nonsilicon atoms and extraframework cations in zeolites. The zeolite database gives the coordinates of the $\mathrm{O}$ atoms and T-sites. ${ }^{73}$ In the case of a pure silica structure, in which all $\mathrm{T}$-sites are occupied with $\mathrm{Si}$ atoms, this is sufficient for the complete structure. In the case where the $\mathrm{Si}$ atoms are replaced by $\mathrm{Al}$, for example, one has to know the aluminum distribution over the T-sites.

The amount of aluminum present in the zeolite structure is usually expressed as the $\mathrm{Si} / \mathrm{Al}$ ratio. Two rules of thumb govern the minimum $\mathrm{Si} / \mathrm{Al}$ ratio that can be obtained and the distribution of the $\mathrm{Al}$ atoms over the T-sites: Löwenstein's rule ${ }^{101}$ and Dempsey's rule. ${ }^{102,103}$ Löwenstein's rule states that the formation of $\mathrm{Al}-\mathrm{O}-\mathrm{Al}$ linkages is energetically highly unfavorable. This rule imposes a lower limit of one on the $\mathrm{Si}$ /Al ratio. Dempsey's rule states that $\mathrm{Al}-\mathrm{O}-\mathrm{Si}-\mathrm{O}-\mathrm{Al}$ linkages are highly unfavorable; hence, aluminum atoms are located preferentially at least three T-sites apart from each other. This rule further limits the $\mathrm{Si} / \mathrm{Al}$ ratio that can be obtained, but Dempsey's rule is less strict than Löwenstein's rule, and at low $\mathrm{Si} / \mathrm{Al}$ ratios it is often violated. ${ }^{104,105}$ While Löwenstein's rule is generally accepted (with a few exceptions in, for example, heulandite (HEU) type zeolites ${ }^{106}$ or sodalite (FAU) synthesized at very high temperatures ${ }^{107}$ ), the validity of Dempsey's rule is still debated.

Fyfe et al. ${ }^{108}$ discovered that there is more that one different type of $\mathrm{Al}$ sites and that the monoclinic form of zeolite ZSM-5 (MFI) has 24 unique T-sites. This discovery gained further importance after Haag et al. ${ }^{109}$ subsequently demonstrated that the aluminum T-sites are the active sites in ZSM-5. Since then, the number of unique T-sites in many other zeolites has been determined. The scattering powers of silicon and aluminum, however, are too close to be able to distinguish between them in X-ray analysis. Many different techniques have been tried to determine the aluminum distributions, including experimental techniques (see, for example, the work by Han et al. ${ }^{110}$ and references therein) such as ${ }^{27} \mathrm{Al}$ and ${ }^{29} \mathrm{Si}$ NMR, MAS NMR, and neutron diffraction.

There are various computational approaches in the literature on how to assign Al atoms to particular T-sites for a given $\mathrm{Si} / \mathrm{Al}$ ratio. The simplest approach is to ignore both

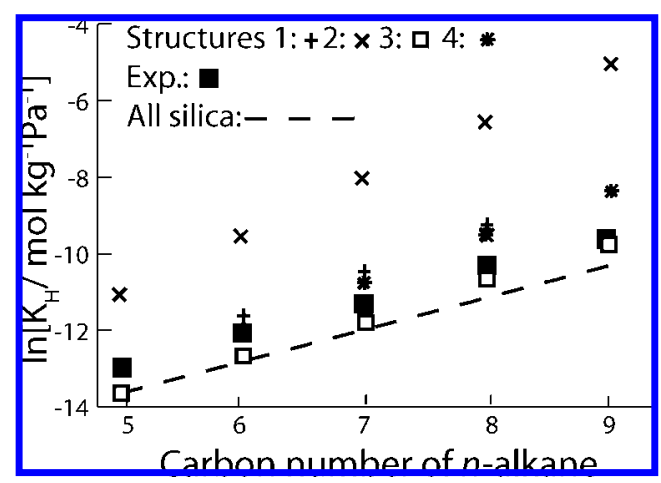

Figure 16. Calculated Henry coefficients as a function of the carbon number for the adsorption of linear alkanes-pentane, hexane, heptane, octane, and nonane-in $\mathrm{H}^{+}\left[\mathrm{AlSi}_{23} \mathrm{O}_{48}\right]-\mathrm{TON}$ at $548 \mathrm{~K}$ compared with experimental data. The closed symbols correspond to the four different distributions of the $\mathrm{Al}$ atoms over the $\mathrm{T}$-sites that are possible given the experimental $\mathrm{Al} / \mathrm{Si}$ ratio. (Figure based on data from ref 122).

Demsey's and Löwenstein's rules and to assume that each T-side is equivalent but carries a charge that depends on the $\mathrm{Si} / \mathrm{Al}$ ratio such that the charge associated with the cations is compensated. This average T-site model has been used in various studies. ${ }^{111-115}$ A slightly more sophisticated approach is either to assume that a T-site can be occupied by an $\mathrm{Al}$ or $\mathrm{Si}$ atom within the restrictions imposed by Löwenstein's and Dempsey's rules ${ }^{116,117}$ or to assign Al atoms to well defined T-sites. ${ }^{118}$

As the position of the cations in dry zeolites is often very close to an aluminum $\mathrm{T}$-site, different distributions over the T-sites can give different locations of the cations. For example, Mellot-Draznieks et al. ${ }^{119}$ showed that different ways to assign $\mathrm{Al}$ to $\mathrm{T}$-sites can give very different distributions of the $\mathrm{Na}^{+}$ions in FAU. Therefore, the location of the cations in the zeolite structure is closely related to the distribution of $\mathrm{Al}$ over the T-sites.

Experimentally, one can obtain from single-crystal X-ray diffraction the possible location of cations. For very few zeolites, this uniquely determines the location of the cations (e.g., LTA), but for most other zeolites, additional assumptions have to be made. For example, in FAU three distinct extraframework positions for $\mathrm{Na}^{+}$cations have been identified. Computational techniques have been used to study the distribution of the $\mathrm{Na}^{+}$cations over these sites (see, for example, ref 120); in most of these simulations the cations were constrained to these extraframework sites. Jaramillo and Auerbach ${ }^{116}$ developed a force field for the cation-zeolite interactions, which allowed them to reproduce the experimental occupancies without constraining the cations to these extraframework sites. Calero et al. ${ }^{121}$ further optimized this potential using the known distribution of cations in LTA.

Calero and co-workers ${ }^{122,123}$ used the observation that the heat of adsorption depends on the location of the Al atoms in the framework as a way to obtain some information on the location of the $\mathrm{Al}$ atoms. Calero and co-workers optimized their potential for those zeolites for which the distribution of $\mathrm{Al}$ over the $\mathrm{T}$-sites had no influence, and with this potential they computed the heats of adsorption for all possible distributions of $\mathrm{Al}$ over the $\mathrm{T}$-sites for a given unit cell. Figure 16 shows that for several systems there is only one distribution that could describe the experimental data. If we subsequently assume that the distribution in a single unit cell is representative of the entire zeolite crystal, the 
results of Calero and co-workers give a prediction of the $\mathrm{Al}$ distribution in these materials.

Sastre et al. ${ }^{124}$ used force field simulations to identify the preferential Al location in ISV (ITQ-7) that gave the lowest energy of the structure. The locations were compared with the experimental IR spectra. Interestingly, a good agreement could only be obtained if in the computations the template molecules that are present in the synthesis were taken into account. This may indicate that the $\mathrm{Al}$ distribution is not the "true" equilibrium distribution for a given structure but might be a kinetically trapped distribution that depends on the details of the synthesis of the material.

\subsubsection{Cation-Adsorbate Interactions}

To study the effect of cations on the adsorption of hydrocarbons, Macedonia et al. ${ }^{125}$ developed a model that describes the interactions with a Lennard-Jones potential combined with a Coulombic term. The parameters of these interactions were partially obtained from quantum calculations. Calero et al. ${ }^{121}$ further optimized these parameters using information on the complete adsorption isotherm. Garcia-Perez et al. ${ }^{219}$ extended these parameters to describe the interactions with $\mathrm{Ca}^{2+}$. Interestingly, for some zeolites, the mobility of the cations seems to be a minor issue, ${ }^{117,126}$ while Calero et al., ${ }^{121}$ however, showed that to understand the adsorption isotherms of hydrocarbons in Faujasite it is essential to allow the cations to move.

A special case is zeolites that contain protons. Calero et al. ${ }^{127}$ used the approach of Dubbeldam et al..$^{94}$ to optimize the Lennard-Jones interactions between an $\mathrm{H}^{+}$ion and a united $\mathrm{CH}_{x}$ group. This approach guarantees a unique set of parameters which resulted in a size parameter $\sigma_{\mathrm{HCH}_{4}}=4.6$ $\AA$, which is significantly larger than the corresponding size parameter of an oxygen united-atom interaction, $\sigma_{\mathrm{OCH}_{4}}=$ 3.47. Calero et al. argue that such a relatively large size parameter can only be explained by water adsorption and that this potential is effectively describing a proton complex with water. This hypothesis is further supported with quantum chemical calculations. One could argue that such an empirical model may have very limited values if extended to other zeolites. In this respect it is encouraging that GarciaPerez and co-workers ${ }^{122,123}$ showed that these potentials also work quite well for other zeolites (FER and TON).

\section{Adsorption of Hydrocarbons in Zeolites}

\subsection{Introduction}

Would the zeolite literature have looked differently if one could have measured the number of molecules adsorbed in the pores of a zeolite at operating conditions? One can argue that the lack of knowledge on adsorption has been one of the main problems in interpreting the experimental results related to the various applications of zeolites. The most obvious point to make is that a proper description of adsorption phenomena is essential for the design of zeolitebased processes for separations or catalysis; the more reliably adsorption is known, the better equipment can be designed. There are quite a number of experimental pure component adsorption isotherms available in the literature. For mixtures, however, there is hardly any experimental data. ${ }^{128,129}$ The reason is that most experiments rely on measuring the weight increase of the zeolite sample. For pure components this can be directly related to the number of adsorbed molecules, but for mixtures one has to carry out additional experiments to determine the change of the composition of the adsorbed phase. This makes the experiments for mixtures significantly more difficult. In addition, we will also argue that a better understanding of mixture adsorption can also result in the discovery of novel separation mechanisms.

A lack of this basic information on the adsorption properties has also had many consequences for our fundamental understanding of these materials. For example, for many years the zeolite literature was haunted with the compensation effect as the explanation for the experimentally observed dependence of, for example, cracking reactions on the chain length of the hydrocarbon. This observation resulted in many theoretical studies that tried to explain how the acidity of zeolites depends on chain length or why there is a chain length dependence on the cracking reaction. Only when Haag ${ }^{130}$ demonstrated that, if one would interpret the reaction kinetics in terms of the concentration of the molecule in the zeolite, instead of in terms of the concentrations of the gas phase, the chain length effect was simply accounting for the fact that in most zeolites longer hydrocarbons adsorb better. Hence, where experimentally it was ensured that the concentration in the gas phase was equal for all experiments, the concentration inside the zeolite was not equal, and once these concentration effects where taken into account, the reaction kinetics turned out to be very similar in all zeolites and nearly independent of chain length.

Adsorption isotherms also play an essential role for the interpretation of diffusion coefficients. From a practical point of view, we are interested in the Fick diffusion coefficient, which relates the diffusion coefficient to a gradient in the concentration. From a thermodynamic point of view, the driving force for diffusion is not a gradient in the concentration but a gradient in the chemical potential. To be able to use both points of view, one needs the (mixture) adsorption isotherms to make the conversion of concentration gradients into chemical potential gradients or vice versa ${ }^{131}$ (see section 6.2 ).

\subsection{Theoretical Aspects}

\subsubsection{Adsorption Thermodynamics}

Before we discuss the simulation results, we first briefly review the more theoretical aspects of adsorption. The thermodynamics of adsorption has been described in many textbooks (e.g., refs 128 and 132). In this review we summarize a few essential points; in particular we focus on the computational aspects.

The adsorption isotherm measures at a given temperature, $T$, the number of adsorbed molecules as a function of the chemical potential (pressure) of the fluid that is in contact with the porous material. In a molecular simulation an adsorption isotherm is conveniently computed in the grandcanonical ensemble in which the temperature, volume $(V)$, and chemical potential of the adsorbed component $(\mu)$ are imposed. For the grand-canonical ensemble the partition function reads

$$
Q_{\mu, V, T}=\sum_{N=0}^{\infty} \frac{\exp (\beta \mu N) V^{N}}{\Lambda^{3 N} N !} \int \mathrm{d} s^{N} \exp \left[-\beta U\left(r^{N}\right)\right]
$$

The connection with the thermodynamics is made via the grand-potential $\Omega$, which is related to the partition function via 


$$
\beta \Omega=-\ln Q_{\mu, V, T}
$$

In this ensemble, the number of adsorbed molecules $(N /$ $V$ ) is an ensemble average

$$
\left\langle\frac{N}{V}\right\rangle=\frac{1}{Q_{\mu, V, T}} \sum_{N=0}^{\infty} \frac{N}{V} \frac{\exp (\beta \mu N) V^{N}}{\Lambda^{3 N} N !} \int \mathrm{d} s^{N} \exp \left[-\beta U\left(\mathbf{r}^{N}\right)\right]
$$

Hence, in a grand-canonical simulation the average number of adsorbed particles is a result of the simulation, from which the adsorption isotherm can be computed directly. To make the connection with experimental data, the chemical potential has to be converted to pressures (see section 2.3).

At low pressure, the Henry regime, the number of adsorbed molecules is proportional to the pressure $P$ :

$$
\rho_{\mathrm{a}}=K_{\mathrm{H}} P
$$

where $K_{\mathrm{H}}$ is the Henry coefficient. Using this equation, one can obtain the Henry coefficient from the low pressure part of the adsorption isotherm. In a molecular simulation, one can also obtain the Henry coefficient directly using the techniques to compute the free energy of the system. To see this, we can write eq 25 in terms of a system of $N-1$ adsorbed particles and an additional particle:

$$
\begin{aligned}
\left\langle\frac{N}{V}\right\rangle=\frac{\exp (\beta \mu)}{\Lambda^{3}} \frac{1}{Q_{\mu, V, T}} \sum_{N=0}^{\infty} \frac{\exp (\beta \mu(N-1)) V^{N-1}}{\Lambda^{3 N-3}(N-1) !} \times \\
\int \mathrm{d} s^{N-1} \mathrm{~d} s \exp \left[-\beta\left(u(r)+U\left(r^{N-1}\right)\right)\right]
\end{aligned}
$$

we can see the term $\int \mathrm{d} s \exp [-\beta u(r)]$ as an ensemble average of a particle that is added to the system but does not interact with it, cf. the Widom test particle method in section 2.4

$$
\left\langle\frac{N}{V}\right\rangle=\frac{\exp (\beta \mu)}{\Lambda^{3}}\left\langle\int \mathrm{d} s \exp [-\beta u(\mathbf{r})]\right\rangle_{\text {test }}
$$

where we have divided the potential into the interactions of $N-1$ particles and the energy of the "test" particle $N, u(\mathbf{r})$. Of special interest is the limit of $\mu \rightarrow 0$, for which the fluid in the reservoir behaves like an ideal gas, and hence

$$
\frac{\exp (\beta \mu)}{\Lambda^{3}}=\beta P
$$

where $P$ is the pressure of the reservoir. If the pressure is also sufficiently low to be in the Henry regime, we can compute the Henry coefficient by adding a test particle at a random position in an empty zeolite:

$$
K_{\mathrm{H}}=\frac{1}{\beta}\langle\exp [-\beta u]\rangle_{\text {test }}
$$

From a computational point of view, this is convenient, as this equation can be used to test the consistency of the results, via a comparison with a direct computation of the adsorption isotherm.

Another important experimental quantity is the isosteric heat of adsorption $q_{\text {is. }}$. In the limit that the reservoir behaves like an ideal gas and the interactions between the adsorbed molecules can be ignored, the heat of adsorption follows directly from the absolute value of the energy difference of a single particle in the ideal gas phase and in the zeolite:

$$
q_{\text {is }}=\left|\langle U\rangle_{z}-\langle U\rangle_{0}\right|+k_{\mathrm{B}} T
$$

where the term $k_{\mathrm{B}} T$ arises from the decrease of the volume or pressure upon adsorption. At this point it is important to note that, at high loadings or nonideal behavior of the bulk fluid, this expression is only an approximation (for more details the reader is referred to refs 133 and 134). The heat of adsorption can be related to the temperature dependence of the Henry coefficient:

$$
\frac{\partial K_{\mathrm{H}}}{\partial \beta}=q_{\mathrm{is}}
$$

From a computational point of view, for some systems it can be more efficient to compute the heat of adsorption directly while for other systems the Henry coefficient requires less CPU-time. Of course, comparing the results from these two method is again an important way of testing the consistency of the simulations.

Myers and Monson ${ }^{134}$ stress that there is an important difference between experiments and molecular simulations. In a molecular simulation one can compute the absolute adsorption and energies. Experiments are carried out in the presence of a gas phase, and hence one measures the excess properties. Converting these excess properties into absolute properties or vice versa requires detailed knowledge on the reference state used in the experiments. ${ }^{134}$ Typically these reference states have been left to the personal preference of the researchers, even though there are strong arguments in favor of adopting the same reference state as for nonelectrolyte dissolution. ${ }^{135,136}$

The properties that can be compared with experiments include the adsorption isotherm, the isosteric heat of adsorption, and the Henry coefficient.

\subsubsection{Adsorption Isotherms}

From a practical point of view it is important to accurately describe the experimental adsorption isotherms. The simplest form is the Langmuir isotherm:

$$
\rho(P)=\rho_{\text {sat }} \frac{b P}{1+b P}
$$

where $P$ is the pressure and $\rho_{\text {sat }}$ is the maximum loading, which can be fitted together with $b$ to the experimental data. In eq 26, it is assumed that a zeolite can be described as a collection of equivalent adsorption sites which are increasingly occupied if the pressure is increased. For some systems this might be a reasonable assumption, but for some zeolites the experimental adsorption isotherms show steps or kinks, which indicate more complex adsorption behavior. This more complex behavior can be described by assuming that there are several different adsorption sites. Often a good description of the pure component isotherms can be obtained with the dual-site Langmuir (DSL) model. ${ }^{50,128}$ In this model the total loading is the sum of the contributions of two sites:

$$
\rho(P)=\rho_{\text {sat }}^{\mathrm{A}} \frac{b_{\mathrm{A}} P}{1+b_{\mathrm{A}} P}+\rho_{\mathrm{sat}}^{\mathrm{B}} \frac{b_{\mathrm{B}} P}{1+b_{\mathrm{B}} P}
$$

where the parameters $b_{\mathrm{A}}$ and $b_{\mathrm{B}}$, and the maximum loadings of the two sites $\mathrm{A}$ and $\mathrm{B}, \rho_{\text {sat }}^{\mathrm{A}}$ and $\rho_{\text {sat }}^{\mathrm{B}}$, have to be fitted against the experimental data. The maximum loading is given by the sum of the maximum loadings of the two sites 


$$
\rho_{\text {sat }}=\rho_{\text {sat }}^{\mathrm{A}}+\rho_{\text {sat }}^{\mathrm{B}}
$$

From a practical point of view it is important to be able to predict the mixture isotherms from pure component isotherm data. The simplest approach is to assume that the different components do not interact and the mixture adsorption is simply the sum of the pure component isotherm weighted with the corresponding partial pressures. If we use a simple Langmuir isotherm, the corresponding multicomponent Langmuir isotherm reads for the loading of component $i$

$$
\rho_{i}\left(P_{i}\right)=\rho_{i, \mathrm{sat}} \frac{b_{i} P_{i}}{1+b_{i} P_{i}}
$$

where $P_{i}$ is the partial pressure of component $i$. The total loading and total pressure are obtained by a summation of all components: $\rho(P)=\sum_{i} \rho_{i}\left(P_{i}\right)$ and $P=\sum_{i} P_{i}$, respectively. In the following sections it will be demonstrated that this multicomponent Langmuir isotherm cannot describe the adsorption phenomena in zeolites correctly.

An alternative approach is to use the ideal adsorbed solution theory (IAST) as developed by Myers and Prausnitz. ${ }^{137}$ The basic equation of IAST theory is the analogue of Raoult's law for vapor-liquid equilibrium, i.e.

$$
P y_{i}=P_{i}^{0}(\pi) x_{i}
$$

where $P$ is the total pressure. $y_{i}$ and $x_{i}$ are the mole fractions of component $i$ in the fluid and the adsorbed phase, respectively. For we can write

$$
x_{i}=\frac{\rho_{i}}{\sum_{j} \rho_{j}}
$$

$P_{i}^{0}(\pi)$ is the pressure for sorption of every pure component $i$, which yields the same spreading pressure, $\pi$, as that for the mixture. The spreading pressure is defined by the Gibbs adsorption isotherm

$$
\frac{\pi A}{k_{\mathrm{B}} T}=\rho^{\mathrm{zeo}} \int_{P=0}^{P=P_{i}^{0}} \frac{\rho_{i}(P)}{P} \mathrm{~d} P
$$

where $A$ is the surface area of the adsorbent, $\rho^{\text {zeo }}$ the density of the zeolite expressed in terms of the number of molecules per unit volume, and $\rho_{i}(P)$ is the pure component isotherm, given by, for example, the dual site Langmuir isotherm, eq 27. The total amount adsorbed is obtained from

$$
\rho_{\text {mix }}=\frac{1}{\sum_{i} x_{i} / P_{i}^{0}(P)}
$$

The set of eqs $27,30,31,32$, and 33 needs to be solved numerically to obtain the loadings of the individual components in the mixture. For a more detailed description, we refer to ref 138 .

In a lattice model the maximum loading is defined exactly; that is, all sites are occupied. In a molecular simulation, however, molecules are not represented as hard spheres and due to the soft interaction one can always add an addition molecule by increasing the chemical potential. In most practical cases, close to saturation the increase in chemical potential needed to add an additional molecule is so large that one obtains a unique saturation loading. However, as pointed out by Skoulidas and Sholl, ${ }^{139}$ this procedure depends on the range of data that are fitted. As an alternative, they proposed to define the maximum loading as the loading where the isosteric heat of adsorption is zero.

At this point it is important to mention that, from a theoretical point of view, the pressure of the fluid plays no direct role in a simulation. The reason is that a zeolite is a solid. Let us imagine the following experiment. We consider such a large zeolite crystal that we can apply very large external forces on this material. We now put this materials in a force "machine" and apply a very large force on say the $x$-direction of the material; unlike the case of a liquid, we can apply a force or pressure that is different in the $x$ and $z$-directions. Such a force may (slightly) change the adsorption isotherm. From a molecular point of view, this is easy to understand, as these forces change the equilibrium positions of the zeolite atoms and hence the adsorbed molecules see a slightly "different" material. From a thermodynamic point of view, the equilibrium conditions are as follows: equal temperature and chemical potential of the molecules inside the zeolite and the molecules of the fluid that is in contact with the zeolite. As in this experimental setup, this pressure is applied only on the zeolite and not on the fluid, and hence the chemical potential and hence the pressure of the fluid have not changed. One would therefore observe a (slightly) different adsorption isotherm for each value of the external pressure. This external pressure should be seen as an additional thermodynamic variable. In a "normal" experiment, the external pressure is equal to the pressure of the fluid reservoir. For such an experiment, changes in the external pressure are too small to have any significant influence on the position of the zeolite atoms, and hence the fluid pressure does not play any role in the adsorption experiment. This situation is very different from that for mesoscopic pores, where the pore diameter is sufficiently large that adsorbed fluids have bulk properties and capillary forces that determine whether or not the fluids will enter the pores.

From a practical point of view, it is important to mention that one can measure the pressure but not the chemical potential, but for the molecules inside the zeolite, it does not matter whether outside the zeolite this chemical potential corresponds to a gas or to a liquid. As in most of the simulations, we assume the zeolite to be rigid, in which case, the external pressure on the zeolite has by definition no influence.

\subsection{Pure Component Adsorption}

\subsubsection{Hydrocarbons MFI}

Silicalite (MFI) is one of the zeolites that is most studied. From an experimental point of view, this is a convenient system, as the material can be synthesized in its all-silica form, and relatively large crystals can be obtained. As a consequence, this material is very well characterized and reliable experimental data have been produced by various different groups. Therefore, adsorption of hydrocarbons in MFI can be seen as the ideal system to develop and test computational approaches. Many simulations studies have been published for the linear alkanes in MFI. ${ }^{18,47,50,51,65,90,91,117,138,140-165}$ A detailed overview of adsorption studies has been compiled some time ago by Fuchs and Cheetham. ${ }^{166}$ At this point we would like to emphasize that besides hydrocarbons the adsorption in MFI of other molecules (e.g., $\mathrm{CO}_{2},{ }^{22,167-169}$ water, ${ }^{170,171}$ etc.) has been studied using molecular simulations. These are, how- 


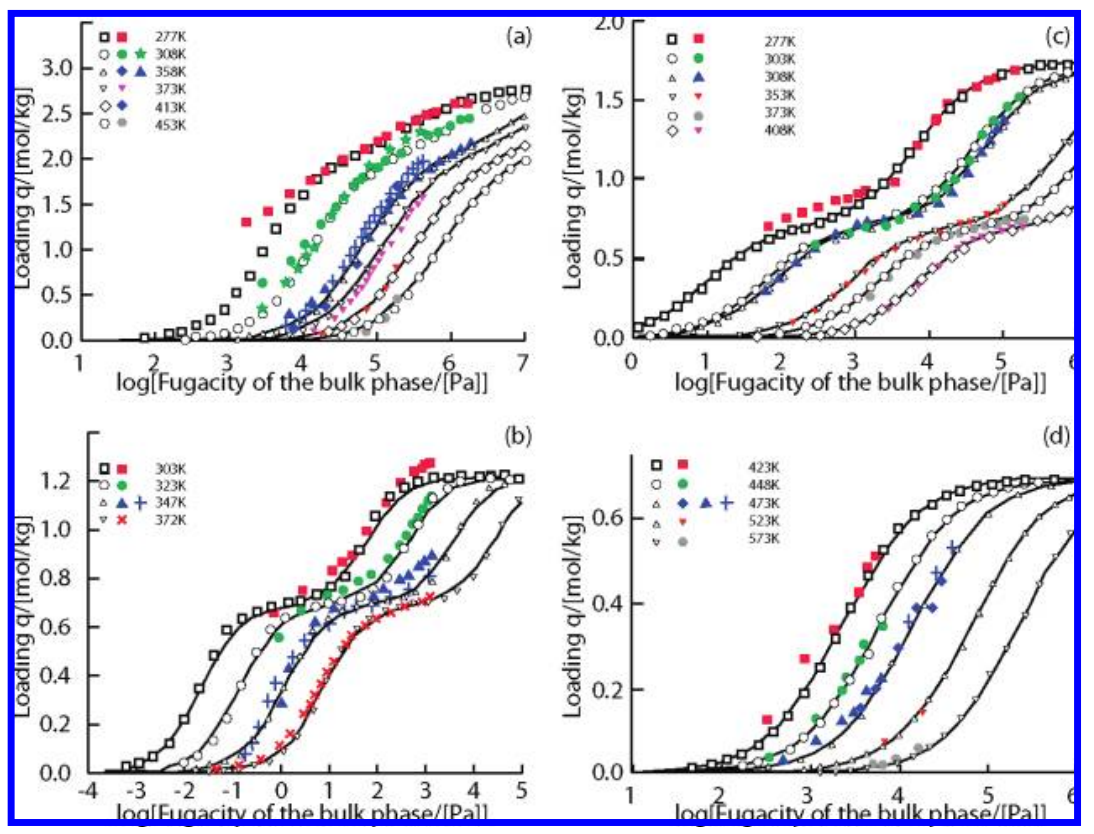

Figure 17. Comparison of the experimental data with the results of molecular simulations. Isotherms of linear alkanes: (a) ethane, (b) $n$-heptane, (c) 2-methylpropane, and (d) 2,2-dimethylbutane in MFI at various temperatures. For the experimental data see refs $173-177$. (Figure based on data from ref 94).

ever, outside the scope of the present review, and we refer to the literature for more information on these systems.

A large number of groups have computed adsorption isotherms of linear alkanes in MFI (see, for example, refs 50, 90, 94, 97, 142, 146, 151, and 163). As most models have been optimized for this system, it is not very surprising that a good description of the adsorption isotherms can be found. For example, De Meyer et al. ${ }^{172}$ studied the maximum loading of $n$-alkanes in MFI both experimentally and by molecular simulations. This study shows a nice agreement between the experiments and the simulations and demonstrates that the very good packing of the long chain hydrocarbons is directly related to the probability of bending and crossing at the intersections of MFI. Figure 17 summarizes the state of the art; over a wide range of temperature and for different hydrocarbons, an excellent agreement with the experimental data can be obtained.

If we look in more detail at the adsorption isotherm of $n$-heptane, we see a plateau in the adsorption isotherm. Interestingly, the early experiments of Dubinin and co-workers ${ }^{178,179}$ for $n$-heptane already showed this plateau, but a discussion on the possible origin of this phenomena was not given. An indication for anomalous behavior of $n$-hexane was also observed by Micke et al., ${ }^{180}$ who could only explain the uptake of $n$-hexane in MFI by assuming that a fraction of the hexane molecules are immobilized in MFI. Their corresponding adsorption isotherms, however, did not show any anomalous behavior. Molecular simulation indicated this behavior might be due to "commensurate" freezing ${ }^{163}$ caused by the fact that the length of a $n$-hexane or $n$-heptane molecule is commensurate with the length of the zigzag channel. Subsequently, experimental adsorption isotherms were published that confirmed the early experimental adsorption isotherms. ${ }^{175,181-183}$ In addition, temperature programmed desorption experiments nicely show a two-step desorption profile for $n$-hexane or $n$-heptane, while for the longer and shorter $n$-alkanes a single desorption step was observed. ${ }^{184-187}$ Interestingly, Makowski and Majda ${ }^{186}$ also observe a two-step adsorption of $n$-heptane in MEL. MEL has a structure very similar to MFI except that the zigzag channels in MEL are straight and therefore form a different, larger, intersection. Also for MEL the distance between the two intersections is similar in length to that for an $n$-heptane molecule, which also opens the possibility for a commensurate-incommensurate transition. It would be interesting to investigate this using molecular simulations in further detail. Ashtekar et al. ${ }^{143}$ showed with FT-Raman that hexane has two distinct adsorption sites. Single-crystal XRD data ${ }^{188}$ confirmed a periodic ordering of the $n$-hexane molecules in the channels of MFI. However, the experimental data of Floquet et al. ${ }^{189}$ indicate a very different mechanism; $n$-heptane has two filling modes. The molecules first fill the straight channels, and only once these are filled do the molecules fill up the zigzag channels. Clearly, these observations are in contrast with most of the previous simulation results that indicate that at low loading there is no preference for the zigzag or straight channels. ${ }^{160,161}$ It would be very interesting to see what the next step in this ongoing discussion will be.

The above example shows that molecular simulation can provide direct insight in the molecular aspects of the sorption behavior. To illustrate this further, let us consider the experimental data ${ }^{173,176}$ for sorption of isobutane in MFI; the sorption isotherm shows a pronounced inflection at a loading of four molecules per unit cell (see Figure 17c). This inflection behavior is nicely reproduced by CBMC simulations. ${ }^{142}$ By actually counting the molecules in the various locations in the MFI structure, straight channels, zigzag channels, and intersections, we can analyze the contributions to the isotherms for each individual location (see Figure 18). It is clear from this figure that the isobutane molecule prefers to locate at the intersections. Up to a system pressure of $1 \mathrm{kPa}$, the isobutane molecules are exclusively located at the intersections. Since these intersections have a maximum capacity of four molecules per unit cell, an excess loading of four molecules per unit cell can only be achieved by "pushing" isobutane into the straight and zigzag channels. This only occurs when the pressure is significantly increased 


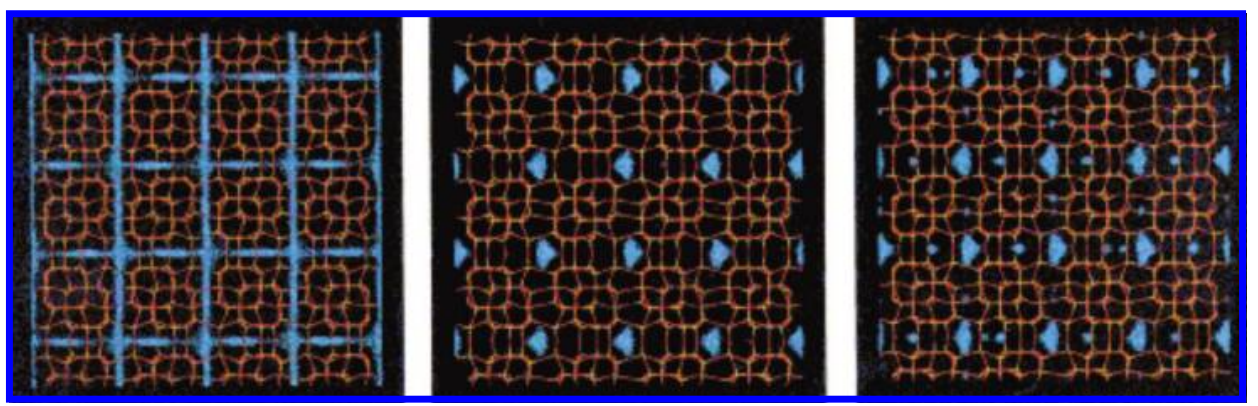

Figure 18. Probability distributions of $n$-butane at $0.1 \mathrm{kPa}$ (left), isobutane at $0.1 \mathrm{kPa}$ (middle), and isobutane at $100 \mathrm{kPa}$ (right) on $\mathrm{MFI}$ at $300 \mathrm{~K}$. The zigzag channels are from the left to the right, and the straight channels are perpendicular to the zigzag channels. These figures were obtained by plotting the centers of mass of the molecules (blue dots) every 500 Monte Carlo cycles. Reprinted with permission from ref 142. Copyright 1998 American Chemical Society.

beyond $10 \mathrm{kPa}$; isobutane demands an extra "push" to locate within these channels. This extra push is the root cause of the inflection behavior. All monobranched alkanes, in the 5-8 $\mathrm{C}$ atom range, are found to exhibit similar inflection behavior at a loading of four molecules per unit cell. The fact that this inflection behavior can be directly related to the structure of the zeolite can conveniently be used to check the accessibility of a zeolite sample.

Dibranched alkanes, typified by 2,2-dimethylbutane (22DMB) (see Figure 17d), also prefer to locate at the intersections of MFI. However, these molecules are much bulkier than monobranched alkanes, and consequently, they cannot be pushed into the channel interiors. The maximum loading of dibranched alkanes, such as 22DMB, in MFI is restricted to four molecules per unit cell.

The inflection behavior of monobranched alkanes in MFI at a loading of four molecules per unit cell, as well as the restriction of the maximum loading of dibranched alkanes to this loading, is a consequence of configurational differences. This configurational entropy effect causes the molecular loadings of hexane isomers in MFI at high pressures to follow the following sorption hierarchy: linear $>$ monobranched $>$ dibranched. ${ }^{152}$

\subsubsection{Other Zeolites}

If we look at the details of adsorption of linear alkanes in MFI, we see, besides some peculiar behavior for hexane and heptane, a "normal" behavior, which can be summarized as follows:

1. The Henry constant increases monotonically as a function of chain length; the longer the chain, the better the adsorption for a given (low) pressure.

2. The heat of adsorption increase almost linearly.

3. The maximum loading is similar for all linear alkanes, i.e., to a first approximation equal to the free volume of MFI times the liquid density of the $n$-alkane.

MFI has a three-dimensional channel-like structure, and there are no barriers that would become apparent if the chain length is increased. The only additional length scale that does play a role is the distance between intersections. This distance is comparable with the size of a hexane or a heptane molecule, which explains why exactly for these molecules a different behavior can be expected. In addition, one can argue that for other molecules (ethane, octane) a similar compatibility issue can occur at different loadings and one may see steps or kinks for these molecules as well.

The fact that MFI forms a three-dimensional channel structure with intersecting tubes becomes important for the branched alkanes. The fact that these bulky molecules have

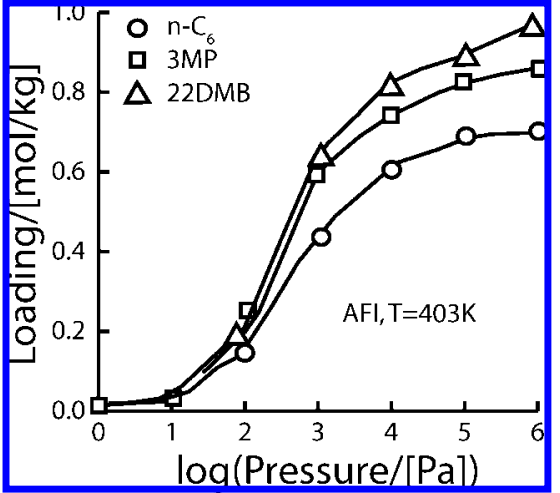

Figure 19. Sorption isotherms for $n$ - $\mathrm{C}_{6}, 3 \mathrm{MP}$ (3-methylpentane), and $22 \mathrm{DMB}$ in AFI at $403 \mathrm{~K}$ determined by CBMC simulations. Figure is based on data from ref 152).

more "leg room" at the intersections makes these sites preferred adsorption sites; adsorption in the channels between the intersection is less favorable and will only occur at sufficiently high pressures. From these arguments we should expect for the branched hydrocarbons a plateau of the adsorption isotherm when all intersections are occupied. In addition, the maximum loading for the different isomers typically follows the order linear $>$ monobranched $>$ dibranched, which reflects a configurational entropy effect ${ }^{152}$ which states that at higher pressure there are more configurations in which the linear molecules can be fitted in the tubes compared to the monobranched and dibranched isomers. On the basis of the above arguments one would expect similar types of adsorption behavior for other three-dimensional channel structures.

For one-dimensional tubelike structures, there are no intersections, and one would expect the behaviors of the linear and branched hydrocarbons to be more similar. The simulations in, for example, $\mathrm{AFI}^{152}$ and TON, ${ }^{94}$ confirm this. An interesting difference compared to the three-dimensional tubelike structures is the maximum loading hierarchy, which is as follows for AFI: linear < monobranched < dibranched. The CBMC simulations of the sorption isotherms at $403 \mathrm{~K}$ are shown in Figure 19. ${ }^{190}$ The reason for the inversion of the hierarchy compared to the case of MFI is again entropic. AFI consists of cylindrical channels of $0.73 \mathrm{~nm}$ radius, which is large enough to accommodate the bulky 22DMB, and thus, there is no configurational penalty for these molecules. However, the effective length of the molecules decreases with an increased degree of branching (see Figure 20), the dibranched molecules are more compact compared to the linear one, and at high pressure you can fit more molecules in a linear tube. At sufficiently high pressures, this entropy 


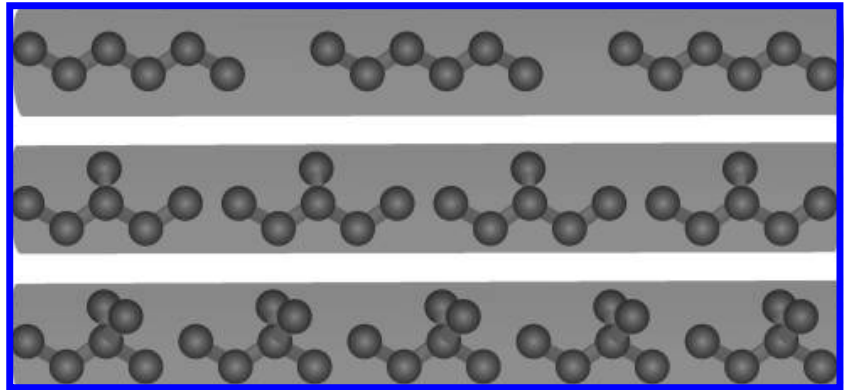

Figure 20. Schematic drawing of the length entropy effect during sorption of $n-\mathrm{C}_{6}, 3 \mathrm{MP}$, and 22DMB in the cylindrical channels of AFI; the higher the branching, the more molecules can be adsorbed per unit length of the zeolite.

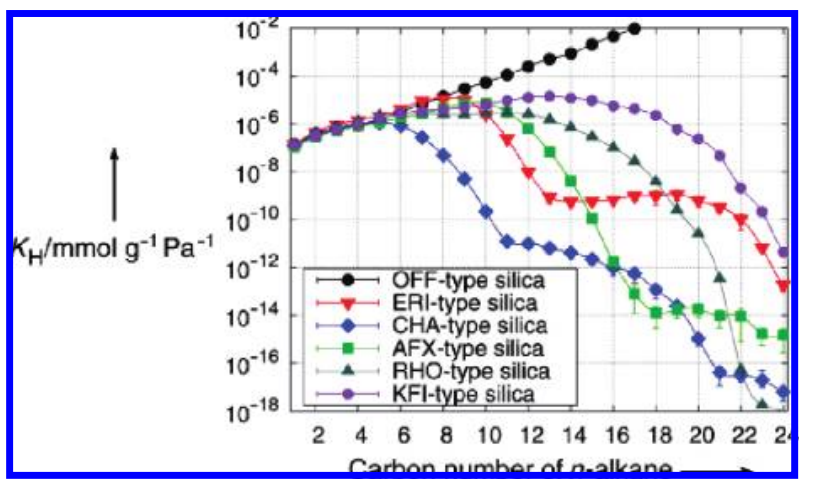

Figure 21. Computed Henry coefficients at $600 \mathrm{~K}$ for various types of zeolites as a function of alkane chain length. Reprinted with permission from ref 194. Copyright 2003 Wiley-VCH Verlag $\mathrm{GmbH} \& \mathrm{Co}$. KGaA.

effect dominates differences in adsorption strengths. These entropy effects will show up again when we discuss the adsorption of mixtures in one of the next sections.

We have seen that interesting behavior can be expected if the zeolite introduces an additional length scale that is compatible with the size of the molecules. If we consider cagelike zeolites, we can expect a different behavior if the size of the cage matches the "effective" size of the molecule. For example, if we start with methane and we increase the chain length, one would see a monotonically increasing Henry coefficient. If we reach the molecular length that optimally fits into the cage, we will have the highest Henry coefficient, and if we further increase the size of the molecule, it will "feel" the limitation of the cage and the molecule has to curl or put part of the chain through the window. Both situations are energetically unfavorable, and hence increasing the chain length will result in a decrease of the Henry coefficient. Figure 21 illustrates this behavior. For the tubelike zeolite OFF, Dubbeldam et al. ${ }^{191,192}$ observe a monotonically increasing Henry coefficient, while for the cagelike zeolites CHA, ERI, AFX, RHO, and KFI, a nonmonotonic behavior is observed, which reflects the differences in cage sizes of these zeolite structures. Complete adsorption isotherms have been computed by Krishna and van Baten. ${ }^{193}$ These simulations show that the adsorption goes in steps with plateaus corresponding to an integer number of molecules per cage. In the next sections, we will discuss the consequences for mixture adsorption behavior and diffusion.

Of course, a classification of cagelike and tubelike structures should be considered with care. For example, AFI can be classified as one-dimensional tubelike structures if one looks at its diffusion behavior, ${ }^{195,196}$ while its adsorption for methane shows the characteristics of cagelike structures. Experimentally, ${ }^{197}$ one can find two steps in the adsorption isotherm at $T=77 \mathrm{~K}$ : one step at a loading of approximately four molecules per unit cell and another step at a loading of six molecules per unit cell. These steps are also found via molecular simulations. ${ }^{196,198,199}$ Simulations predict that these steps should disappear if the temperature is raised above $T$ $=100 \mathrm{~K}$, suggesting a phase transition to occur in the pores of the zeolite. The molecular explanation is that AFI has a very corrugated tubelike structure of which the corrugations can also be seen as cages. Further simulations of hexane isomers in AFI have been carried out by Fox et al. ${ }^{165}$

Another important aspect is that the topology may depend on the molecules that are adsorbed. Various studies deal with the adsorption of $n$-alkanes in FER. FER has ten-ring channels that are interconnected with eight-ring cages. These cages form relatively narrow side channels that are of comparable dimensions to the alkanes. Using ${ }^{13} \mathrm{C}$ NMR, van Well $^{200,201}$ showed that the short molecules adsorb in both the ten-ring and eight-ring channels, while $n$-hexane only adsorbs in the ten-ring channels. For $n$-pentane a transition was observed: at low loading, $n$-pentane preferentially adsorbs in the ten-ring channels, while, at high loading of $n$-pentane, the eight-ring channels also become occupied. This observation was also confirmed by FT-Raman spectroscopy. ${ }^{202}$ From a computational point of view, these results are very interesting, as this system is very sensitive to the details of the size parameters. For example, the unitedatom force field of Smit and Siepmann ${ }^{160,161}$ uses as Lennard-Jone size parameter $\sigma_{\mathrm{CH}_{2} \mathrm{O}}=3.64 \AA$. Van Well et $\mathrm{al}^{203}$ showed that with this force field the adsorption of $n$-pentane in FER was significantly underestimated compared to the experimental data, ${ }^{203,204}$ while the size parameter used by June et al., ${ }^{47} \sigma_{\mathrm{CH}_{2} \mathrm{O}}=3.364 \AA$, overestimated the adsorption. Ndjaka et al. ${ }^{205}$ used the parameter set of Vlugt et al. ${ }^{50}$ which has a slightly smaller size parameter $\left(\sigma_{\mathrm{CH}_{2} \mathrm{O}}\right.$ $=3.60 \AA$ ) compared to the one used by Smit and Siepmann, but also these parameters underestimate the adsorption in FER. Pascual and co-workers showed ${ }^{206}$ that by using an anisotropic united atom model ${ }^{95,96}$ a much better agreement with the experimental data could be obtained. These results are important, as they may suggest a serious limitation of the united atom force field. This suggestion in ref 206 was followed up by Liu and co-workers. ${ }^{122,123,207}$ Liu et al. showed that, with the united-atom force field of Dubbeldam et al., ${ }^{94,97}$ which uses a $\sigma_{\mathrm{CH}_{2} \mathrm{O}}=3.58 \AA$, an excellent agreement with the experimental data could be obtained. Interestingly, this agreement could only be obtained if the model included the effect of the acid sites in FER. This suggests that aluminum-free FER type silica should exhibit adsorption isotherms significantly different from those of FER-type zeolites.

The good agreement of the simulated adsorption isotherms of the linear and branched hydrocarbons with the experimental ones is an encouraging result. Several molecular simulation studies have been published that all reproduce the adsorption of linear hydrocarbons in silicalite very well using different types of force fields. ${ }^{94,95,97,208}$ Experimental adsorption isotherms are not readily available for a given zeolite at a given condition. These results show that one can get a reasonable estimate from a molecular simulation. However, it is important to point out that most simulations have been performed for silicalite for which the potentials have been developed as well. Unfortunately, there are not 


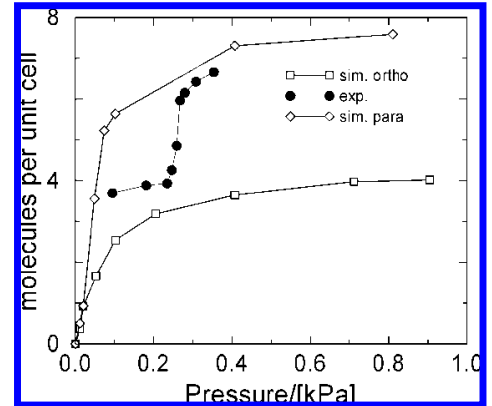

Figure 22. Comparison of the simulated ${ }^{212}$ (open symbols) and experimental adsorption ${ }^{210}$ isotherms of $p$-xylene in silicalite. The simulations use the para and ortho structures of silicalite-1.

many experimental adsorption isotherms of other all-silica zeolites. It is therefore not known how accurate these simulations extrapolate to other zeolites.

\subsubsection{Flexible Lattice}

At this point it is important to mention that most simulations use a rigid zeolite lattice. To see the limitation of this assumption, let us consider the transition which is observed in the adsorption of benzene or xylene isomers in silicalite. ${ }^{209-211}$ Olson et al. observed a step in the adsorption isotherm for $p$-xylene at $70^{\circ} \mathrm{C}$, a plateau at a loading of four molecules per unit cell with saturation at six molecules per unit cell (see Figure 22). Van Koningsveld showed that at four molecules per unit cell a structural transition of the zeolite framework from the ortho to the para structure occurs. ${ }^{211}$ From a molecular simulation point of view, this is a very challenging system to study. A very pragmatic approach was adopted by Snurr et al., ${ }^{212}$ who have computed the adsorption isotherms of $p$-xylene in both the ortho and the para structures of silicalite (see Figure 22). For both the ortho and the para structures, a simple Langmuir isotherm is observed. The maximum loadings for the ortho and para structures were four and eight molecules per unit cell, respectively. Comparison with the experimental data shows that the jump in the adsorption isotherm is consistent with a change in the structure.

A similar behavior phase transition was observed for the adsorption of benzene in silicalite. ${ }^{213}$ For this system the agreement between experiments and the simulations of Snurr et al. ${ }^{212}$ was good at high temperatures but less satisfactory at low temperatures. This discrepancy motivated Clark and Snurr ${ }^{214}$ to study the adsorption of benzene in silicalite in detail. Their study showed that the adsorption isotherms of benzene are very sensitive to small changes in the structure of the zeolite. Also, these calculations were performed with a rigid zeolite and one would expect that the zeolite structure would "respond" to the presence of these molecules. Clark and Snurr point out that this requires simulation with a model of a zeolite with accurate flexible lattice potentials.

As shown above in the case of the aromatics in silicalite, the fit is very tight and can even induce a phase transition that has a large effect on the adsorption. For molecules that do not have a tight fit in the zeolite framework, the assumption of a rigid zeolite appears to be very reasonable. Indeed, Vlugt and Schenk ${ }^{215}$ systematically investigated the effect of flexibility of the zeolite on the adsorption of hexane in MFI and only at high loading could an effect be observed. Of course, a real zeolite is flexible and it might be possible to demonstrate the limitations of the assumed rigidity also for the case of linear alkanes. However, for as long as these limitations have not been discovered, it is very difficult to justify the enormous increase in CPU-time associated with a flexible lattice.

\subsubsection{Cation Effects}

Experiments show that the sorption properties of a zeolite depend on framework $\mathrm{Si} / \mathrm{Al}$ ratio and the type of cation used to compensate the increase of the negative charge of the framework. ${ }^{18,216,217}$ Several molecular simulation studies on the effect of cations on the properties of adsorbed molecules have been published. ${ }^{117,118,218}$ For the adsorption properties of alkanes, the influence of cations is a combination of two competing factors. The presence of a cation can strengthen the adsorption because of the favorable interactions with the alkanes or may block adsorption sites, which results in a weaker adsorption. At high loading, the blocking of adsorption sites dominates, resulting in a lower loading with increasing cation contents. At low loading, it depends on the systems whether the cations cause blocking or favor the adsorption. For MFI the cations tend to be located in the intersections, so that they block the favorable adsorption sites for the branched alkanes while for the linear alkanes the cations favor the adsorption, resulting in a larger Henry coefficient. ${ }^{117}$ Garica-Perez et al. ${ }^{219}$ emphasize the importance of the total amount of cations in a zeolite structure. For example, for decane in the Linde type A (LTA)-structure at low temperature, the adsorption can change orders of magnitude if the cation composition is changed. As in many experimental studies, the cation content is not very well know, making it difficult to compare the experimental results.

Accurate force fields for the adsorption of hydrocarbons in zeolites with $\mathrm{Na}^{+}$cations have been developed by Calero et al. ${ }^{121}$ This force field gives a very good description of the adsorption of the $n$-alkanes in FAU- and MOR-types ${ }^{126}$ of zeolites and has been extended to include $\mathrm{Ca}^{2+}$ ions. ${ }^{219}$ Garcia-Sanchez et al. ${ }^{220}$ demonstrate that this force field gives a very good description of the experimental data of methane, ethane, and propane adsorption in LTA. Granato et al. ${ }^{221}$ show, however, that this force field results in an overestimation of the adsorption of methane, ethane, and propane in sodium-containing LTA. Granato et al. ${ }^{221}$ developed a force field that gave a better representation of the experimental data of this group. It would be very interesting to investigate whether this force field also gives an accurate description of other zeolite structures that have not been included in the fitting.

Wender et $\mathrm{al}^{222}$ proposed a force field that includes polarization. Calero et al. ${ }^{121}$ introduce an effective polarization by fitting the Lennard-Jones interactions between the cations and the atoms of the alkane. From a fundamental point of view, there is no discussion that polarization is the correct way of describing the alkane-cation interactions. From a computational point of view, however, polarization requires special algorithms to deal with the induced dipoles self-consistently. ${ }^{12}$ Wender et al. ${ }^{222}$ argue that in zeolites these second order terms can be ignored, in which there is no need for a special algorithm. With these assumption the difference between the approaches of Calero et al. and Wender et al. reduces to a change of the range of the attractive interactions, i.e., from $r^{-6}$ to $r^{-4}$, respectively. More importantly, Calero et al. observed that it was essential to allow the cations to move freely in the zeolite to obtain a good agreement with experimental data, while Wender et 
al. argue that, as the cations did not move in their model, this was an artifact of the $r^{-6}$ potential. Clearly, it would be very interesting to see whether this issue can be addressed experimentally.

\subsection{Adsorption of Mixtures}

Almost all applications of adsorption involve mixtures, ${ }^{129,223}$ yet the number of experimental studies on adsorption of mixtures is very limited due to the difficulty of experimentation. Whereas for pure components the number of adsorbed molecules can be determined accurately by simply measuring the weight increase of the zeolite sample, for mixtures one has to carry out additional experiments to determine the composition inside the zeolite.

Compared to pure components, fewer simulation studies have been published on mixtures of hydrocarbons. Computation studies include mixtures of (nobles) gases, ${ }^{148,224,225}$ alkanes, ${ }^{19,50,138,146,151,154,221,226-237}$ or aromatics. ${ }^{113,147,238,239}$ Detailed knowledge on the composition inside the pores of the zeolites is essential to understand mixture diffusion. In addition, most chemical reactions involve mixtures, and knowing the concentration in the zeolite at the reaction conditions would be very useful. Here we will try to demonstrate that molecular simulations combined with a correct theoretical description can be very useful to obtain a very reasonable estimate of the mixture adsorption isotherm if no experimental data is available.

\subsubsection{Adsorption Mechanisms}

There are a few general observations one can make to help understand the mixture behavior

-At sufficiently low partial pressures one can assume that the probability that the molecules interact with another molecule of the same or other component is very small. Hence, the adsorption simply follows from the Henry coefficient of the pure components:

$$
n_{\mathrm{tot}}=n_{i}+n_{j}=K_{\mathrm{Hi}} p_{i}+K_{\mathrm{H} j} p_{j}
$$

where $p_{i}$ and $K_{\mathrm{H} i}$ are the partial pressure and Henry coefficient of component $i$, respectively.

-At higher pressures the interactions between molecules play an increasingly important role. At the very high pressure end of the adsorption isotherms, the dominant driving force is entropic in nature. The zeolite favors the composition which optimally fills the available space.

If we apply the above to a mixture of two components in say a tubular zeolite structure, one would expect the following scenario. As the interactions with the zeolite usually increase with the size of the molecule, one would expect the larger molecule to be preferentially adsorbed at low pressures. At high pressures there are simply more ways in which one can pack the smaller molecule ${ }^{240}$ and, hence, because of entropic reasons, one would observe a reversal of the selectivity.

\subsubsection{Mixtures Adsorption Studies}

Let us start with a relatively simple case of a mixture of two $n$-alkanes with different lengths in MFI. Figure 23 shows that the Henry coefficient of the $n$-alkanes in MFI is a monotonically increasing function; hence, at low pressure the selectivity is always toward the longest component. Figure 24 shows the mixture isotherms for a $50 \%-50 \%$

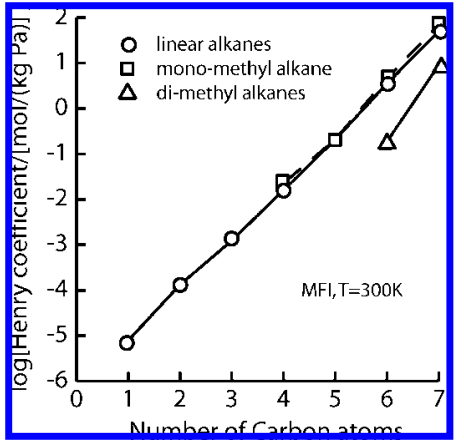

Figure 23. Henry coefficients of alkanes in MFI at $300 \mathrm{~K}$, calculated using CBMC simulations. Figure based on data from ref 138 .

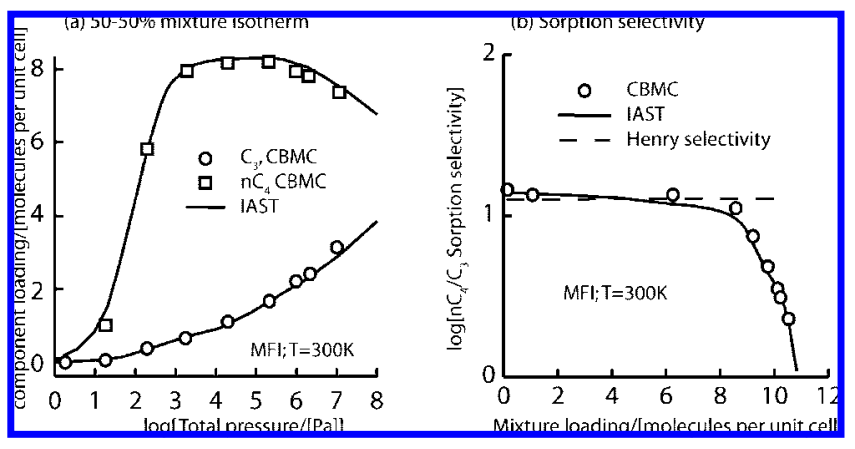

Figure 24. (a) Sorption loadings of an equimolar binary mixture of $\mathrm{C}_{3}$ and $n-\mathrm{C}_{4}$. (b) $n-\mathrm{C}_{4} / \mathrm{C}_{3}$ sorption selectivity. The continuous lines represent the predictions of the IAST. (Figure based on data from ref 152).

mixture of $n$-propane and $n$-butane in MFI at $300 \mathrm{~K}$. Indeed, at low pressure the selectivity is toward the larger component. As the mixture loading increases beyond 8, the predictions made by the Henry coefficients fail completely as the sorption selectivity decreases dramatically to values just above unity. Near saturation loadings, the vacant spaces in the zeolite are more easily occupied by the smaller propane molecule. A similar scenario has been observed for different mixtures of linear hydrocarbons in MFI, ${ }^{146,154,231,241} \mathrm{AFI},{ }^{229}$ and FAU ${ }^{231}$ and also for noble gases in zeolite LTA. ${ }^{224}$ However, if the difference in carbon number between the two components is large, one may have to increase the pressure to extreme values before this entropy effect sets in.

Denayer et al. ${ }^{242}$ studied the adsorption of mixtures of $n$-alkanes using breakthrough experiments with a HPLC column packed with MFI zeolites. The results show a preferential adsorption of the longest alkanes for $\mathrm{C}_{8} / \mathrm{C}_{12}, \mathrm{C}_{9} /$ $\mathrm{C}_{13}$, and $\mathrm{C}_{9} / \mathrm{C}_{11}$, while the shortest alkane preferentially adsorbed for $\mathrm{C}_{14} / \mathrm{C}_{15}$ and $\mathrm{C}_{15} / \mathrm{C}_{16}$ mixtures. A crossover was observed for $\mathrm{C}_{6} / \mathrm{C}_{10}$ and $\mathrm{C}_{5} / \mathrm{C}_{7}$ mixtures. Figure 25 shows that these experimental observations can be reproduced quantitatively using CBMC simulations by Chempath et al. ${ }^{235}$

An important practical example is the separation of alkane isomers. This system is of considerable practical importance for various petrochemical applications. The traditional approach is to use a zeolite to sieve the bulkier branched alkanes from the smaller linear hydrocarbons. The disadvantage of this process is that because of the sieving function relatively small pore zeolites are used, which limit the fluxes and therefore require relatively large separation equipment. It would therefore be interesting whether one can use differences in adsorption to separate these isomers. Figure 23 shows that in MFI the Henry coefficients of the linear 


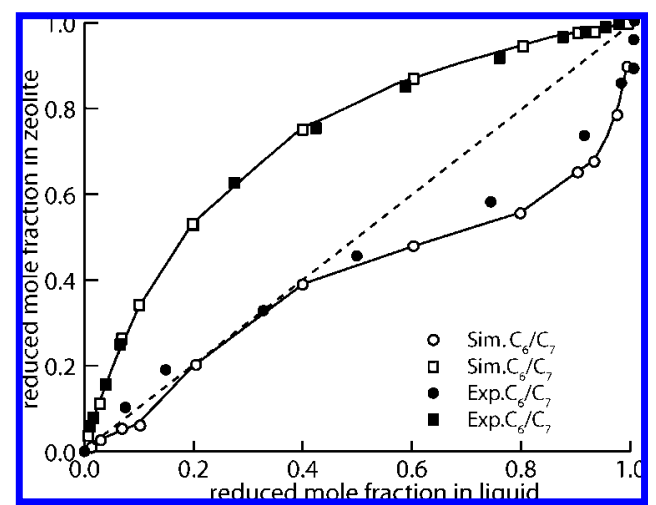

Figure 25. Comparison of the experimental (exp from Denayer et al. ${ }^{242}$ ) and simulation ( of mixutures of $n$-alkanes $\left(\mathrm{C}_{6} / \mathrm{C}_{7}\right.$ and $\left.\mathrm{C}_{8} / \mathrm{C}_{9}\right)$ in MFI. These reduced mole fractions are calculated on the basis of the number of molecules of adsorbing alkanes, where the $x$-axis is the mole fraction in the liquid and the $y$-axis is the corresponding mole fraction in the zeolite. (Figure based on data from ref 235).

and branched isomers are very similar. Also for onedimensional zeolites, such as AFI or MOR, the Henry coefficients predict little or no selectivity.

At high pressures, however, entropy effects set in again. Figure 26(a) shows the CBMC simulations ${ }^{50,159}$ for sorption of a 50-50 mixture of $n-\mathrm{C}_{6}$ and 3 methylpentane (3MP) in MFI. At low pressure the selectivity can be estimated from the Henry regime. It is interesting to note the maximum in the loading of $3 \mathrm{MP}$ at about $100 \mathrm{~Pa}$. When the pressure is raised above $100 \mathrm{~Pa}$, the loading of $3 \mathrm{MP}$ reduces virtually to zero. The $n-\mathrm{C}_{6}$ molecules fit nicely into both straight and zigzag channels whereas the $3 \mathrm{MP}$ molecules are preferentially located at the intersections between the straight channels and the zigzag channels (see section 5.3.1). Below a total loading of four molecules per unit cell, there is no real competition between $n-\mathrm{C}_{6}$ and $3 \mathrm{MP}$. The $n-\mathrm{C}_{6}$ locates within the channels and $3 \mathrm{MP}$ at the intersections. When all the intersection sites are occupied, further adsorption of 3MP requires an extra "push". Entropically, it is more efficient to obtain higher mixture loadings by "replacing" the 3MP with $n-\mathrm{C}_{6}$; this configurational entropy effect is the reason behind the curious maxima in the 3MP loading in the mixture. Krishna and van Baten $^{243}$ observed similar effects for benzene/alkane mixtures. It is interesting to compare these results with the studies of $\mathrm{Lu}$ et al. ${ }^{236,237}$ for mixtures of $n$-butane and isobutane in ISV, BEA, and BOG. These zeolites also have a three-dimensional pore structure. The difference with MFI is that their tube diameters are larger, i.e.,12-rings compared to the 10-rings in MFI. Since the tubes are larger, the intersection are not the preferred adsorption sites of the branched isomer and this entropy effect does not occur. Lu et al. ${ }^{237}$ did observe a similar behavior for mixtures of $n$-butane and isobutane in TER and MEL, which have a similar 10-ring structure to that of MFI.

The $n-\mathrm{C}_{6} / 3 \mathrm{MP}$ sorption selectivity is plotted in Figure 26(b). We see that the sorption selectivity increases from near-unity values for pressures below $100 \mathrm{~Pa}$ to values of around 50 near saturation loadings. At the time these simulations were published, there were no experimental data available to directly compare with these simulation results. However, there were some experimental data that were consistent with these observations. For example, Funke et $\mathrm{al}^{244}$ observed that in the case of the hexane and 3-methylpentane the pure component fluxes through an MFI based

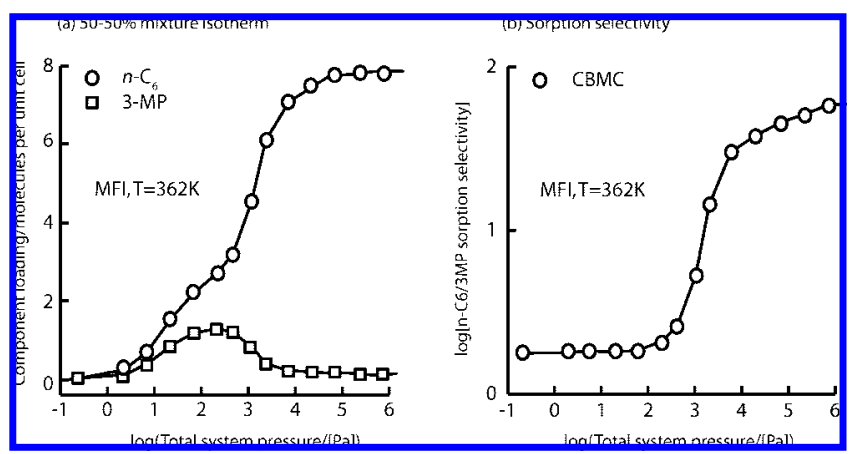

Figure 26. (a) CBMC simulations of the 50-50 mixture isotherm for $n-\mathrm{C}_{6} / 3 \mathrm{MP}$ at $362 \mathrm{~K}$ in MFI. (b) Sorption selectivity as a function of total system pressure. (Figure based on data from ref 152).

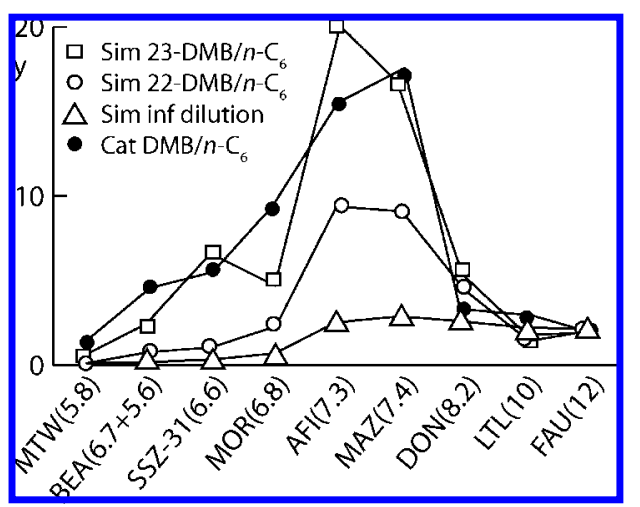

Figure 27. Ratio of 2,3 dimethylbutane and $n$-hexane and ratio of 2,2-dimethylbutane and $n$-hexane in various zeolites as a function of the pore diameter at high and low loading. (Figure based on data from ref 247).

membrane are nearly identical but in a mixture the permeation is dominated by hexane. This observation can be explained on the basis of the simulation results, since at the conditions of the experiments hexane is preferentially observed. The experiments of $\mathrm{Yu}$ et al. ${ }^{245}$ provide direct evidence that the simulations correctly predict the preferential absorption of the linear isomer. It is interesting to compare the previous results with experimental data. Uguina et al. ${ }^{246}$ studied the adsorption of a mixture of $\mathrm{C}_{13}$ isomers in the zeolite structures MFI, BEA, and AEL. The experimental results confirm the preferential adsorption in MFI of the linear alkanes at the expense of the branched isomers. Fox and Bates $^{234}$ show that if cyclohexane is added at low temperatures, the adsorption is dominated by hexane, while at high temperatures (above $600 \mathrm{~K}$ ) cyclohexane is preferentially adsorbed.

In the case of MFI the bulky branched molecules can be packed less conveniently compared to the linear alkanes, and therefore the linear molecules are expelling the branched isomers at high pressures. One can also have the reverse situation. Let us consider several different zeolites, with each having a one-dimension channel topology but with varying tube diameter. Schenk et al. ${ }^{247,248}$ studied the adsorption of mixtures of linear and mono- or dibranched alkanes. Figure 27 shows the ratio of the branched and linear isomers for the various zeolites at low and high pressures. At low loading the selectivity is, for most zeolites, toward the linear molecule; for some zeolites a slight preference for the branched isomers is found. Molecular simulations show that at low pressures the energy difference between the linear and branched isomers is very small for those zeolites in which the diameter is sufficiently large to accommodate the 


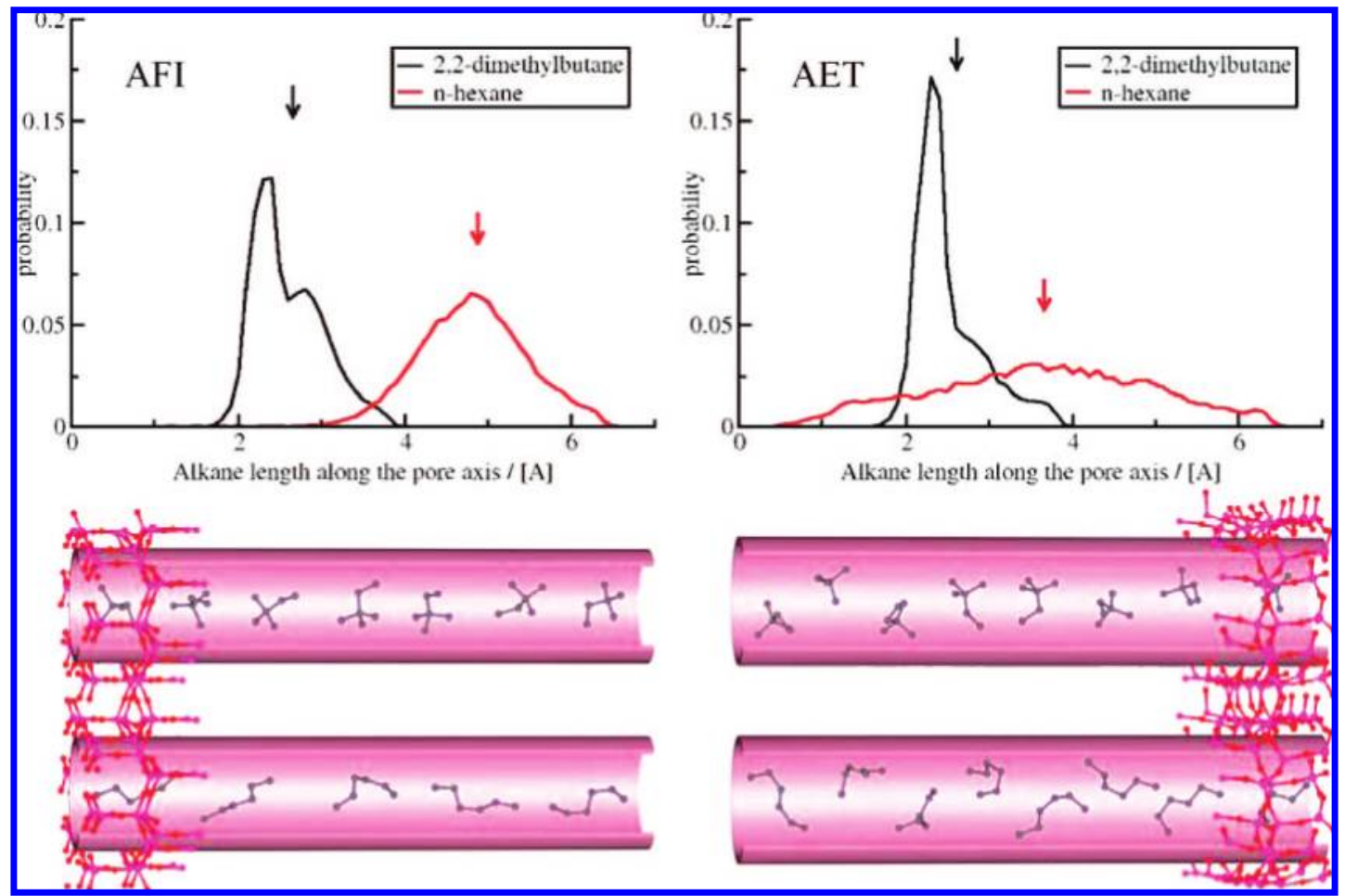

Figure 28. Length entropy effect; comparison of the effective length of 2,2-dimethylbutane and $n$-hexane in the AFI (left) and AET (right). In the top figures the effective length, which is defined as the largest distance between two atoms of a molecule, is projected on an axis parallel to the axis of the channel. The bottom figure compares some snapshots of the systems. Reproduced with permission from ref 247. Copyright 2002 Wiley-VCH Verlag GmbH \& Co. KGaA.

branched molecules. ${ }^{248}$ At high loading we observe a large optimal selectivity depending on the pore diameter. Lu et al. ${ }^{236,237}$ computed the adsorption isotherms of mixtures of $n$-butane and isobutane in the one-dimensional structures TON, MOR, and CFI. Lu et al. also observed, depending on the pore diameter an optimal selectivity at high pressures.

The molecular explanation is again an entropy effect. At high loading the isomers compete for space and the more compact dibranched isomers pack more efficiently, but only if the zeolite is confining these isomers to a linear conformation. In Figure 28 this length entropy effect is illustrated more quantitatively. At high pressure the molecule that has effectively the smallest size is preferentially adsorbed.

A very interesting case is for mixtures in cagelike zeolites. In section 5.3.2 we have seen that for the $n$-alkanes the Henry coefficient does not increase monotonically as a function of chain length but can decrease if the chain is longer than the optimal length. However, these Henry coefficients are for empty cages; at higher loading, the molecules will compete for space in the cage. The calculations of Krishna and van Baten ${ }^{193}$ indicate that the entropy effect that separates the small $\left(\mathrm{nC}_{4}\right)$ from the large alkanes $\left(\mathrm{nC}_{6}\right)$ occurs in $\mathrm{AFX}$ at relatively low pressures compared to those for MFI or MOR.

\subsubsection{Effect of Cations}

The selectivity for a given zeolite can be further optimized by changing the number or type of cations. Experiments report changes in the selectivity for xylene isomers in $\mathrm{Y}$ (FAU) if the type of cation is changed. ${ }^{249}$ Also, membrane permeation experiments on mixtures of linear and branched alkanes in MFI show a change of selectivity if the type of cation is changed. ${ }^{250}$ Lachet et al. ${ }^{113,238,239}$ used molecular simulations to study the effect of cations on the adsorption selectivity. The simulations showed a reversal of the selectivity if $\mathrm{Na}^{+}$is exchanged by $\mathrm{K}^{+}$. The differences in selectivity are related to a combination of differences in size and location of these cations. This results in a completely different adsorption behavior. Beerdsen et al. ${ }^{117,251}$ showed that since cations in MFI preferentially occupy the intersections, which correspond to the preferred adsorption sites of the branched isomers, increasing the number of cations therefore favors the adsorption of the linear alkanes.

\section{Diffusion in Zeolites}

\subsection{Introduction}

Understanding the diffusion of molecules adsorbed in the pores of a zeolite is important for almost all applications of zeolites. Separations are often based on differences in diffusion coefficients of the components, and in catalytic application, diffusion of the molecules to the active site can be a rate limiting step. From a scientific point of view, this is a very important issue, as the diffusion behavior of molecules in confined geometries is very different from their bulk behavior. Diffusion in a confined geometry introduces some new phenomena such as single-file diffusion and resonant diffusion.

Experimentally, it is a challenge to accurately measure diffusion coefficients. ${ }^{252}$ Depending on the experimental techniques, diffusion coefficients may vary by orders of magnitude. ${ }^{252,253}$ The reasons why there are such differences are as yet poorly understood, and joint research projects have been started to investigate these differences in detail. ${ }^{254}$

From a molecular simulation point of view, computing diffusion coefficients is challenging, as diffusion coefficients may vary as much as 10 orders of magnitude. There has been significant progress in the development of novel 
computational techniques that can deal with these large ranges in diffusion coefficients. The most straightforward way is to compute a diffusion coefficient of an adsorbed molecule directly from a molecular dynamics simulation. This is, however, limited for those systems in which the diffusion coefficient is sufficiently large such that reliable statistics can be obtained within reasonable amounts of CPUtime. For those systems for which the diffusion coefficient is very small, special techniques have been developed in which one uses rare event simulation methods.

Most practical applications are not limited to pure components at infinite loading. It is therefore important to obtain insights as to how to extrapolate these zero-loading results to higher loading and to mixtures. In addition, in molecular simulations the self-diffusion coefficient is conveniently computed, while in practical applications one is often interested in the transport diffusion coefficient. We will illustrate how molecular simulations can be used to obtain molecular insights in these diffusion coefficients. The topic of diffusion in nanoporous materials is a very active area of research, and various reviews have been published. $28,30,76,255-257$

\subsection{Theoretical Aspects}

\subsubsection{Which Diffusion Coefficient?}

A confusing aspect about diffusion is that one can find several diffusion coefficients. ${ }^{131,252}$ Most practical applications involve the transport diffusion or Fick diffusion coefficient $\left(D^{\mathrm{t}}\right)$. The Fick diffusion coefficient is closely related to the intuitive definition of a diffusion coefficient as a measure of the flow of material if a concentration gradient is imposed on a system. If we define $J$ as the (macroscopic) flux of particles induced by the application of a concentration gradient

$$
\mathbf{J}(c)=-D^{t}(c) \nabla c
$$

where $c$ is the concentration (in molecules per unit volume).

Whereas experimentally it is convenient to impose a concentration gradient, thermodynamics, however, states that the driving force for the diffusion is the gradient in the chemical potential

$$
\mathbf{J}(c)=-\frac{L(c)}{k_{\mathrm{B}} T} \nabla \mu=-D^{\mathrm{c}}(c) \nabla \mu
$$

where $L(c)$ is the single-component (Onsager) transport coefficient and $\mu$ the chemical potential. $D^{\mathrm{c}}$ is called the collective, corrected, or Maxwell-Stefan diffusion coefficient. ${ }^{131}$

In an NMR experiment, a different diffusion coefficient is measured. In a so-called pulse field gradient (PFG-NMR) experiment, one labels a particle and the attenuation of the signal is directly related to the self-diffusion coefficient $\left(D^{\mathrm{s}}\right)$. The self-diffusion coefficient is a measure of the average mean-square displacement a labeled particle has made in a given time interval.

Figure 29 shows an example of these diffusion coefficients for hydrogen molecules in the zeolite FAU. In the limit of zero loading, the differences between these diffusion coefficients disappear, while for high loading the differences between them can be large. To compare the results of the various techniques, it is therefore very important to know the exact relations between the various diffusion coefficients. Here, we give a compact introduction; a more complete

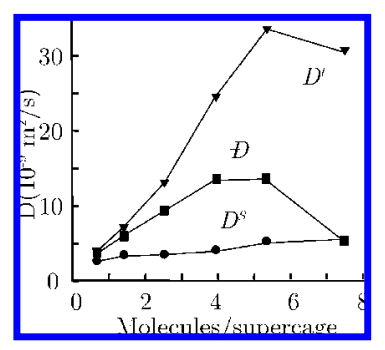

Figure 29. Transport $\left(D^{\mathrm{t}}\right)$, Maxwell-Stefan $(D)$, and self-diffusion coefficients $\left(D^{\mathrm{s}}\right)$ of hydrogen in $\mathrm{NaX}$ (FAU) as a function of loading. (Figure based on data from ref 258).

description can be found in the excellent book by Kärger and Ruthven ${ }^{252}$ or in several reviews. ${ }^{28,131}$

To link the Maxwell-Stefan diffusion coefficient to the transport diffusion coefficient, we need to convert a gradient of the concentration into a gradient of the chemical potential. For this, let us define a thermodynamic correction factor $\Gamma$

$$
\Gamma \equiv \frac{1}{k_{\mathrm{B}} T c} \frac{\partial \mu}{\partial \ln (c)}
$$

With this factor, we can write

$$
\nabla \mu=\Gamma \nabla c
$$

we can now separate the transport diffusion coefficient into a thermodynamic and a diffusion part

$$
D^{\mathrm{t}}(c)=\Gamma D^{\mathrm{c}}(c)
$$

or in terms of the Onsager coefficient

$$
D^{\mathrm{t}}(c)=L(c) \Gamma
$$

In the limit of zero loading we can assume ideal gas behavior, and the thermodynamic factor, $\Gamma$, approaches unity and, hence, the transport diffusion coefficient is equal to the Onsager transport coefficient. At higher loading, this approximation does not hold and one has to know the adsorption isotherm to compute the thermodynamic factor.

For a molecular simulation it is important to relate the diffusion coefficients to the microscopic properties of the system. The Onsager transport coefficients can be expressed in terms of the autocorrelation function of the velocities of the particles

$$
L=\frac{1}{3 V} \int_{0}^{\infty}\left\langle\sum_{i=1}^{N} \mathbf{v}_{i}\left(t^{\prime}\right) \sum_{j=1}^{N} \mathbf{v}_{j}(0)\right\rangle \mathrm{d} t^{\prime}
$$

where $\mathbf{v}_{i}(t)$ is the velocity of the particle at time $t$ and the summation runs over all $N$ particles in the system.

In the limit of zero loading, adsorbate-adsorbate interactions become negligible and the velocity correlations between different particles in eq 40 have little influence on the diffusion coefficient. We can rewrite eq 40 as the sum of velocity correlations between the same particle and between two different particles:

$$
\begin{aligned}
& L(c)=\frac{1}{3 V} \sum_{i=1}^{N} \int_{0}^{\infty}\left\langle v_{i}\left(t^{\prime}\right) v_{i}(0)\right\rangle \mathrm{d} t^{\prime}+ \\
& \frac{1}{3 V} \sum_{i=1}^{N} \sum_{j \neq i}^{N} \int_{0}^{\infty}\left\langle v_{i}\left(t^{\prime}\right) v_{j}(0)\right\rangle \mathrm{d} t^{\prime}
\end{aligned}
$$

The first part is also referred to as to the self-diffusion or tracer diffusion coefficient 


$$
D^{\mathrm{s}}(c)=\frac{1}{3 N} \sum_{i=1}^{N} \int_{0}^{\infty}\left\langle v_{i}\left(t^{\prime}\right) v_{i}(0)\right\rangle \mathrm{d} t^{\prime}
$$

This diffusion coefficient is measured in NMR experiments. In the limit of very low loading the transport diffusion coefficient and self-diffusion coefficient are equal.

\subsubsection{Multicomponent Diffusion}

In a mixture diffusion of a given component, the driving force for diffusion is not only the gradient of the chemical potential of this particular component, but also a gradient in the chemical potential of the other components can influence the diffusion. Here we only give a very short summary of the essential aspects; a more detailed discussion can be found in the literature $28,259-261$

The generalization of eq 35 to mixtures reads

$$
(\mathbf{J})=-\frac{1}{k_{\mathrm{B}} T}[\mathbf{L}](\nabla \mu)
$$

where the components of $(\mathbf{J})$ are the fluxes of the various components and $[\mathbf{L}]$ is a matrix with the Onsager transport coefficients. For a binary mixture, eq 43 reads

$$
\left(\begin{array}{l}
\mathbf{J}_{1} \\
\mathbf{J}_{2}
\end{array}\right)=-\frac{1}{k_{\mathrm{B}} T}\left[\begin{array}{ll}
L_{11} & L_{12} \\
L_{12} & L_{22}
\end{array}\right]\left(\begin{array}{l}
\Delta \mu_{1} \\
\Delta \mu_{2}
\end{array}\right)
$$

where we have used the Onsager reciprocal relation $L_{12}=$ $L_{21}$.

In pure components, we can only compute the velocity autocorrelation function, but in a mixture, we can correlate the velocity of component 1 with all other components, including component 1 . For mixtures, the Onsager transport coefficients can therefore be expressed in terms of the correlation function of the velocities of the particles of components 1 and 2 .

$$
L_{12}=\frac{1}{3 V} \int_{0}^{\infty}\left\langle\sum_{i=1}^{N_{1}} \mathbf{v}_{i}^{(1)}\left(t^{\prime}\right) \sum_{j=1}^{N_{2}} \mathbf{v}_{j}^{(2)}(0)\right\rangle \mathrm{d} t^{\prime}
$$

where $\mathbf{v}_{i}^{(1)}$ is the velocity of particle $i$ of component 1 and $N_{1}$ is the number of particles of component 1 . Similarly to the pure component case, one can define the self-diffusion coefficients for each component, $D_{11}^{S}$, via $L_{11}$.

The extension of the pure component Fick's transport equation to mixtures is

$$
(\mathbf{J})=-\left[\mathbf{D}^{\mathrm{t}}\right](\nabla c)
$$

where $\left[\mathbf{D}^{\mathbf{t}}\right]$ is the diffusion matrix. The relation between the Onsager transport coefficients and the Fick diffusion matrix can be obtained from equating eqs 43 and 46, giving

$$
\left[\mathbf{D}^{\mathrm{t}}\right]=\frac{1}{k_{\mathrm{B}} T}[\mathbf{L}]\left[\frac{\partial \mu}{\partial c}\right]
$$

where $\partial \mu_{1} / \partial c_{2}$ is a component of the matrix $\partial \mu / \partial c$.

The generalization of the Maxwell-Stefan diffusion coefficients is obtained via the flux, which can be written as

$$
(\mathbf{J})=-\frac{1}{k_{\mathrm{B}} T}[\mathbf{L}][\mathbf{B}]^{-1}[\Gamma](\nabla c)
$$

in which $[\Gamma]$ is the thermodynamic matrix with elements

$$
\Gamma_{12} \equiv \frac{c_{1}}{k_{\mathrm{B}} T c_{2}} \frac{\partial \mu_{1}}{\partial \ln \left(c_{2}\right)}
$$

Compared to pure components, we see that because of the cross terms $\Gamma_{12}$ and $\Gamma_{21}$ the thermodynamic factor is much more complex. These cross terms reflect the fact that in a mixture changes in the concentration of component 2 may affect the chemical potential of component 1 . Therefore one needs to know the compete mixture isotherms to compute all components in these equations.

The matrix [B] contains the mixture Maxwell-Stefan diffusion coefficients and has as elements

$$
[\mathbf{B}]=\left\{\begin{array}{c}
B_{11}=\frac{1}{D_{1}}+\sum_{1 \neq 2} \frac{c_{2}}{D_{12}} \\
B_{12}=-\frac{c_{1}}{D_{12}}
\end{array}\right.
$$

\subsection{Computational Aspects}

An important difference between the self-diffusion and transport diffusion coefficients is that self-diffusion is a single-particle property, while transport diffusion is a collective property. This implies that in a simulation of a system of $N$ particles one obtains a single data point for the transport diffusion coefficient and at the same time $N$ data points for the self-diffusion coefficient, which makes the transport diffusion coefficient more difficult to compute. The expressions for the diffusion coefficients, eqs 41 and 42, involve time dependent properties. These are conveniently computed from a molecular dynamics simulation.

\subsubsection{Molecular Dynamics Simulations}

For a three-dimensional system, we can determine the selfdiffusion coefficient for each dimension separately. For the $x$-direction, eq 42 becomes

$$
D_{x}^{\mathrm{s}}=\int_{0}^{\infty}\left\langle v_{x}(t) v_{x}(0)\right\rangle \mathrm{d} t
$$

where $v_{x}(t)$ is the $x$ coordinate of the velocity of a tagged methane molecule at time $t$. Since the tagged particle could be any particle in the system, we can average over all particles in our system to increase the accuracy of our calculation. Or alternatively, we can use the mean-squared displacement, which gives for the $x$-direction

$$
D_{x}^{\mathrm{s}}=\frac{1}{2} \lim _{x \rightarrow \infty} \frac{d}{d t}\left\langle[x(t)-x(0)]^{2}\right\rangle
$$

where $x(t)$ is the $x$ coordinate of methane at time $t$. In a similar way we can compute the diffusion coefficients in the $y$ - and $z$-directions, from which we can compute the overall diffusion coefficient $D$

$$
D=\frac{D_{x}+D_{y}+D_{z}}{3}
$$

For the collective coefficient, $D^{\mathrm{c}}$, in the $x$-direction, we obtain from eqs 39 and 40

$$
D_{x}^{\mathrm{c}}(c)=\frac{1}{N} \int_{0}^{\infty} \sum_{i=1}^{N} \sum_{j=1}^{N}\left\langle v_{x i}\left(t^{\prime}\right) v_{x j}(0)\right\rangle \mathrm{d} t^{\prime}
$$

Similar expressions can be written for the $y$ - and $z$ directions, and eq 53 can be used to compute the overall diffusion coefficient. From a computational point of view 
there is no difference whether we use the mean-square displacement or the velocity autocorrelation function; they give identical results.

To compute the transport diffusion coefficient from the collective diffusion coefficient, one needs to know the adsorption isotherm to compute the thermodynamic correction factor $\Gamma$, given eq 38. An alternative is to compute this factor directly from the structure factor. ${ }^{262}$

An important practical issue is that, for the computation of the diffusion coefficients, eqs 52 and 54 , one needs a sufficiently long simulation such that the extrapolation to infinite time can be made reliably. In addition, the system needs to be sufficiently large such that the simulations are sampling the relevant macroscopic regime.

Sufficiently long implies that the total simulation time should be so long such that the mean-squared displacement is truly linear in time, which is the signature for diffuse behavior. A simple way to check whether one has diffuse behavior is to make a $\log -\log$ plot of the mean squared displacement as a function of time. At the very short time scale, one can see the ballistic behavior $\left(t^{2}\right)$, which should change into a linear behavior for longer time scales. If one does not get a line with slope 1 , the behavior may not be diffusive, for example, if one has a one-dimensional channel in which the molecules cannot pass each other. In such a case, molecules move as a single file through the zeolite and one would see a slope of 0.5 instead of 1 (see section 6.6.3).

The question whether the system is sufficiently large to compute a diffusion coefficient sufficiently accurately has been addressed by Hoogenboom et al. ${ }^{262}$ by considering a wave vector dependent formulation of the Green-Kubo expression that relates the decay of density fluctuations to the diffusion coefficient. In the conventional approach only the macroscopic limit $k \rightarrow \infty$ is used. Hoogenboom et al. considered the wavelength dependent diffusion coefficient for diffusion in a one-dimensional channel parallel to the $z$-direction

$$
D\left(k_{x}\right)=\int_{0}^{\infty}\left\langle\sum_{l, m} \frac{1}{N} v_{l x}(\tau) v_{m x}(0) e^{i k_{x}\left(x_{l}(\tau)-x_{m}(0)\right)}\right\rangle \mathrm{d} \tau
$$

This equation can be used to compute the diffusion coefficient as a function of the wavenumber. The diffusion coefficient is related to (macroscopic) density fluctuations, and in a system with periodic boundary conditions these fluctuations are limited to wavelengths of half the boxes size. If at low wave vectors the results of eq 55 show a plateau, one is guaranteed that the system is sufficiently large that the relevant large wavelength fluctuations in the density are sampled. This plateau value therefore gives direct information on the minimum size of the simulation box. The wave vector dependent form of eq 52 has been derived by Tepper and Briels, ${ }^{263}$ who also emphasized the importance of using a correct thermostat to simulate at constant temperature (see also section 2.2).

Whereas the self-diffusion coefficient can be efficiently computed from a conventional molecular dynamics simulation, in contrast, the transport diffusion coefficient requires significantly more CPU-time. Several alternative techniques have been developed. These alternative techniques prepare the system in a nonequilibrium configuration. Maginn et $\mathrm{al}^{264}$ introduced the gradient relaxation molecular dynamics in which the diffusion coefficient is computed from the diffusion equation that has been fitted to the relaxation of a concentration gradient. The disadvantage of this technique is that it involves a larger number of particles and is therefore computationally very demanding. Maginn et al. ${ }^{264}$ also introduced a computationally less demanding technique, the external field nonequilibrium molecular dynamics technique, in which the response of the system to an external field is computed. A color field acts as the driving force for diffusion, and the resulting flux is computed. A third technique, the dual control volume grand canonical molecular dynamics ${ }^{265,266}$ (see section 2.5), involves two reservoirs in the zeolite that are maintained at different values of the chemical potential. This steady state nonequilibrium situation results in a flux that can be related directly to the transport diffusion coefficient. Arya et al. ${ }^{267}$ made a detailed and critical comparison of these techniques and concluded that the external field nonequilibrium molecular dynamics technique is the most efficient. An important issue in these nonequilibrium simulations is that the system is in the linear response regime, and one often has to perform several simulations to ensure that this is indeed the case. The equilibrium technique based on wave vector dependent Green-Kubo expressions as proposed by Briels and co-workers ${ }^{262,263}$ has an important advantage that this check comes straight out of the simulations.

\subsubsection{Rare Event Simulations and Kinetic Monte Carlo}

In section 3.2 the basic principles of rare event simulations are described in detail for a single molecule at infinite dilution. Such a simulation gives the hopping rate of the molecule jumping over a free energy barrier that separates one low energy configuration from another. From this hopping rate the diffusion coefficient can be computed analytically or via a kinetic Monte Carlo scheme. This technique has been applied to compute the diffusion coefficient for a large number of systems. ${ }^{51,150,194,268-273}$

These studies differ in the way the free energy of the barrier is computed. June et al. ${ }^{274}$ used a numerical technique to compute the partition function. Such an approach can be used for a small number of degrees of freedom (atoms or spherical molecules). For more complex molecules one can use constrained dynamics to compute the potential of mean force from which the free energy can be computed via integration $^{268,270}$ or free energy methods such as configurational-bias Monte Carlo for long chain alkanes. ${ }^{51,194}$ Nagumo et al. ${ }^{275}$ demonstrate how one can obtain these free energy profiles from simulations at high temperatures using non-Boltzmann sampling techniques which ensure that the bias introduced by simulating at the "wrong" temperature is removed exactly.

In Figure 3 we have sketched an ideal situation where one observes two minima well separated via a single barrier. In practice one may observe several local minima that are separated by barriers that vary in height. ${ }^{51,268}$ If all barriers are sufficiently high, one can compute the hopping rate for each barrier separately and obtain the overall hopping rate from one absolute minimum to another, which one has to identify the rate determining barrier or decompose the hopping process (see Figure 30). Auerbach ${ }^{30}$ has written a detailed review on the application of this technique and related methods to study the jump dynamics.

In section 2.6 we have assumed that the zeolite lattice has a simple geometry, in which the hopping rates in the $x$-, $y$-, and $z$-directions are identical. For only a few zeolites is the crystal structure symmetry so high that the diffusion coef- 


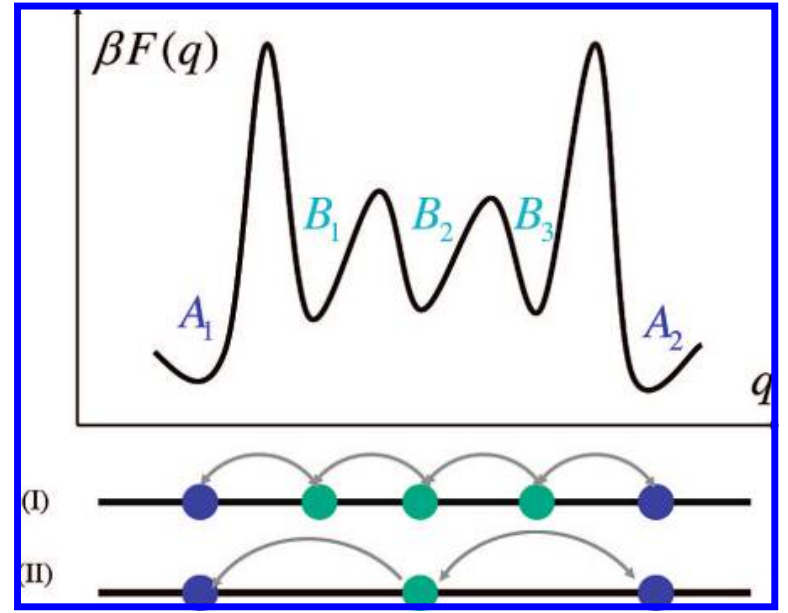

Figure 30. Mapping of a free energy profile onto a hopping model. The free energy as a function of the order parameter shows two absolute minima $A_{1}$ and $A_{2}$ and three local minima $B_{1}, B_{2}$, and $B_{3}$. The free energy barriers are $\beta \Delta F_{B \rightarrow B}$, and the barriers separating the local minima will be crossed many times on the time scale of a jump from $A_{1}$ to $B_{2}$; in such a case mapping II is appropriate, in which the three local minima are lumped into a single site, while if the barriers are of comparable height, mapping I should be used and the hopping rate for each subprocess.

ficients are equal in all directions. In the most general case one has to determine the diffusion coefficient in each direction separately. In a molecular simulation this is straightforward, but experimentally this is difficult since it requires relatively large single crystals. ${ }^{276-278}$ Most experimental studies are on powder samples, which implies that an average diffusion coefficient is measured over all directions.

Most of the rare event simulations are performed in the limit of infinite dilution. At higher concentrations guest-guest interactions can be important. For example, a molecule can occupy a lattice site and hence prevent another molecule from jumping to this site. This effect can be incorporated in a kinetic Monte Carlo simulation. More complicated is the case that the presence of other guest molecules changes the hopping rate. Techniques to take into account these effects using a simulation approach have been developed by Tunca and Ford ${ }^{279,280}$ or using an approximate theory by Auerbach and co-workers. ${ }^{281-284}$

An alternative approach is to assume a distribution of lattice sites in a zeolite and to obtain the hopping rates from fitting to experimental data or to assume certain values and investigate the effect of changes in the hopping rate. These simulations are particularly useful to obtain insight into the mechanism of diffusion. Applications of these simulations have been reviewed by Keil et al. ${ }^{28}$

A rigorous extension of transition theory beyond high loading has been proposed by Dubbeldam and co-workers. ${ }^{285-287}$ In this technique one computes the free energy barrier of a tagged particle. The contributions of the other particles in the systems are included in this free energy barrier and in the recrossing rate. From this hopping rate one can compute the self-diffusion coefficient directly. This diffusion coefficient corresponds exactly with the one that would be obtained from a molecular dynamics simulation if the assumptions underlying the rare events simulations hold, i.e., that once a particle has hopped over a free energy barrier it remains sufficiently long in the free energy minimum such that it can fully equilibrate before jumping over the next barrier. This technique, however, only provides the selfdiffusion coefficient.

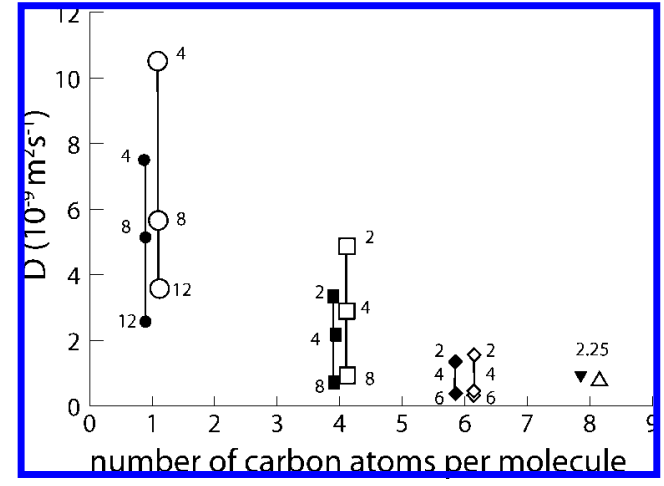

Figure 31. Self-diffusion coefficient of the linear alkanes as a function of carbon number; the closed symbols are the results of rigid lattice simulation, and the open symbols are from flexible ones. The numbers correspond to the loading in molecules per unit cell. (Figure based on data from ref 295).

\subsubsection{Effect of a Flexible Lattice}

Barrer and Vaughan ${ }^{288}$ studied the adsorption of gases in sodalite (SOD) and observed that noble gas could enter through the narrow windows of SOD at high temperatures and remained trapped inside the material at low temperatures. On the basis of these observations Barrer and Vaughan concluded that the zeolite framework must have some flexibility to allow the large molecules to enter. Deem et al. ${ }^{289}$ used more sophisticated models to study the flexibility of the zeolite, and these simulations support the conclusions of Barrer and Vaughan.

From a computational point of view it is attractive to assume that the zeolite atoms do not move, since it reduces the CPU-time by an order of magnitude. It is, however, important to understand how (the lack of) flexibility can influence the diffusion behavior of the molecules. Several studies have been published that compare the diffusion in a flexible and rigid lattice. Kopelevich and Chang ${ }^{290}$ studied the effect of lattice vibration on the diffusion of the noble gases in SOD using transition state theory. In particular for the larger noble gases Kopelevich and Chang observed that in a flexible zeolite the diffusion coefficient is much higher compared to the case in a rigid one. Demontis and co-workers ${ }^{76,93,291,292}$ studied the diffusion of methane. Comparison with a rigid zeolite lattice showed that the overall diffusion coefficient was little influenced. The underlying velocity autocorrelation function, however, did show significant differences, but these differences compensate while taking the integral to obtain the diffusion coefficient. Bouyermaouen and Bellemans ${ }^{141}$ observed a faster diffusion of $n$ - and isobutane in a flexible MFI compared to a rigid one. Fritzsche and co-workers ${ }^{293,294}$ observed little influence for methane in LTA ${ }^{293}$ while for MFI at low loadings the diffusion coefficient of methane was higher in a flexible lattice. ${ }^{294}$ Dubbeldam et al. ${ }^{194}$ computed an increase of the free energy barrier for propane in ERI, suggesting a decrease of the diffusion coefficient compared to the case of a rigid lattice. Leroy et al. ${ }^{295}$ systematically studied the influence of lattice flexibility for the $n$-alkanes in MFI. Figure 31 shows that the flexibility increases the diffusion coefficient, but it also shows that this increase can be very small for the longer hydrocarbons and at high loading. The maximum increase (ca. 30\%) is found for methane at low loading. Leroy et al. ${ }^{295}$ and Zimmermann ${ }^{296}$ have shown that for the same system, depending on which 
model is used for the zeolite interactions, either an increase or a decrease of the diffusion coefficient is observed.

From the examples we have seen that, depending on the system, a flexible zeolite lattice can either decrease or increase the diffusion coefficient. An increase of the diffusion coefficient can be expected for tight fitting molecules, and the flexibility of the zeolite lattice can facilitate the crossing of a barrier. For less tightly fitting molecules the flexibility of the zeolite may result in fluctuations of a window which are "circumference-preserving"; for example, a circular window will become an ellipse in which one axis is smaller compared to the circle. In such a system one may envision a decrease of the diffusion coefficient.

From the above discussion one can conclude that the effect of zeolite flexibility is complex. From a simulation point of view it is an important question whether one needs a flexible model to predict the diffusion coefficient sufficiently accurately. Clearly, from a chemical point of view a fully flexible model is a more realistic description of a zeolite. However, from a computational point of view such a more realistic description only translates into a better description if we have a sufficiently accurate description of the zeolite-zeolite interaction. The fact that two commonly used zeolite models give a qualitatively different prediction of the effect of lattice vibrations illustrates this point. ${ }^{296}$ In addition, another important factor which makes it difficult to compare diffusion coefficients from flexible and nonflexible zeolite models is that one often uses the same zeolite-adsorbate interactions. These interactions are usually obtained from fitting to experimental adsorption data, and the optimal parameters may differ depending on whether a flexible or rigid zeolite is used.

\subsection{A Simple Lattice Model}

It is instructive to consider the simple lattice model introduced in section 2.6 (see Figure 8). In the limit of low loading one can see the diffusion of a molecule to be equivalent to a random walk on a lattice, and we can use this simple model to develop some understanding of the temperature and loading dependence of the diffusion coefficient.

\subsubsection{Temperature Dependence}

If we use our a random walk model as a reference, we can easily derive the temperature dependence of the zeroloading diffusion coefficients. If we assume that the hopping rate is determined by a molecule that has to jump over a free energy barrier $\Delta F$, transition state theory states that the hopping rate is given by (cf. eq 23)

$$
k=k_{0}(T) \exp \left(-\Delta F / k_{\mathrm{B}} T\right)
$$

If we assume that the free energy barrier is independent of temperature, we obtain for the temperature dependence of the diffusion coefficient of our ideal random walk on a cubic lattice

$$
\ln (D)=\ln \left[k_{0}(T) a^{2}\right]-\frac{\Delta F}{k_{\mathrm{B}} T}
$$

which is the typical Arrhenius type of behavior that is associated with activated diffusion. Figure 32 shows that this Arrhenius behavior has been observed in many different systems.

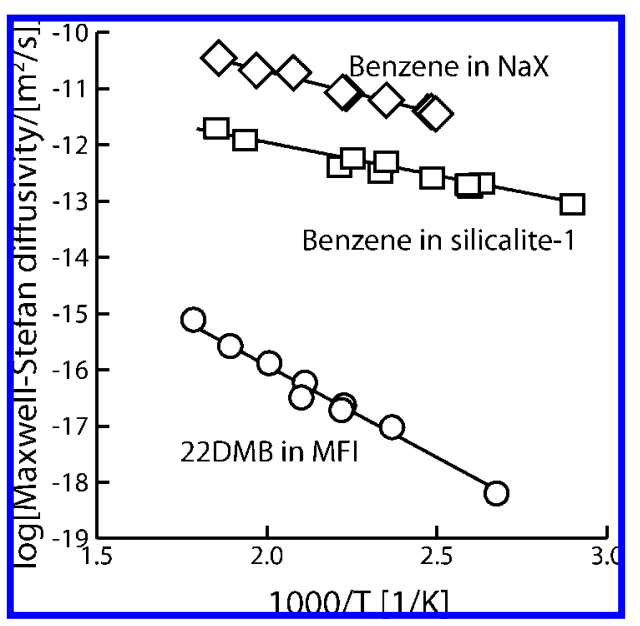

Figure 32. Temperature dependence of the Maxwell-Stefan diffusion coefficients of various molecules in different zeolites. (Figure based on data from ref 241).

Deviations from the expected Arrhenius behavior have been observed experimentally ${ }^{297,298}$ for branched alkanes in MFI at high pressure (see Figure 33). The explanation of this non-Arrhenius behavior is that the experiments have been carried out at fixed pressure, and as the temperature increases, the loading in the pores of the zeolites decreases. In section 5.3.1 we have shown that these branched alkanes prefer to occupy the intersection of the channels, and only if all intersections are occupied (4 molecules per unit cell) will the molecules also start to occupy the space in between intersections. Figure 33 shows that the temperature at which all intersections are occupied corresponds exactly with the temperature below which the slope of the temperature dependence of the diffusion coefficient changes. Clearly, the presence of molecules in between the intersection changes will result in a different hopping rate and explains the nonArrhenius behavior.

\subsubsection{Loading Dependence}

Let us consider the lattice model of Figure 8. We assume that particle-particle interactions do not change the hopping rates but only prevent two particles from occupying the same site. The consequence is that the hopping rate is reduced by a factor $(1-\theta)$, giving a loading dependence of the selfdiffusion coefficient

$$
D^{s}=D^{s}(0)(1-\theta)
$$

If we assume that the adsorption isotherm is described with a simple single-site Langmuir isotherm (see eq 26), the thermodynamic correction term is given by eq 36

$$
\Gamma^{\text {Langmuir }}=\frac{1}{1-\theta}
$$

A typical example of such a system that can be described with such a simple model is 2 -methylhexane in MFI. ${ }^{299}$ 2-Methylhexane preferentially adsorbs at the intersections of MFI, and diffusion can be seen as hopping from one intersection to another. One can see that, for this system, our assumptions that the molecules do not influence the hopping rate, other than setting the hopping rate to zero if a molecule attempts to jump to an occupied site, are very reasonable, as these intersections are sufficiently far apart that the molecules do not influence each other. This hopping rate has been determined by rare events simulations ${ }^{51}$ and 


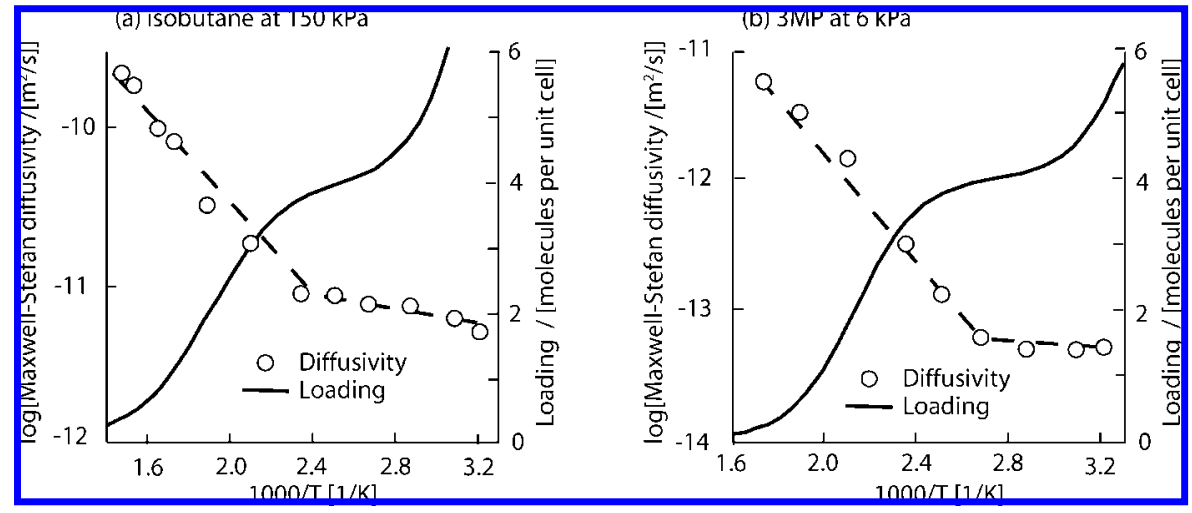

Figure 33. Non-Arrhenius behavior of the diffusion coefficients of isobutane (a) and 3-methylpentane (b) in MFI. (Figure based on data from ref 241).

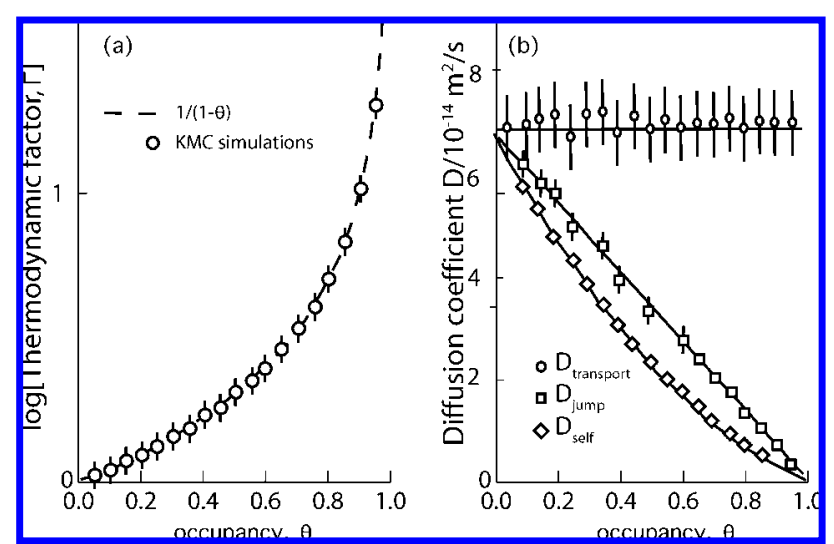

Figure 34. (a) Thermodynamic factor of 2-methylhexane in MFI as a function of loading. (b) Self-, jump- (=collective), and transport-diffusion coefficients for 2-methylhexane. (Figure based on data from ref 299).

the loading dependencies from kinetic Monte Carlo simulations. Figure 34 shows that the loading dependence of the self-diffusion coefficient is compensated by the thermodynamic factor, giving a loading independent transport diffusion coefficient.

Random Walk: Effect of Intermolecular Interactions. In real systems intermolecular interaction can alter the free energy barriers associated with the molecular jumps. Reed and Ehrlich $^{300}$ developed a simple model to take the effects of these forces into account. This model has been extended to zeolites by Krishna and co-workers. ${ }^{301,302}$ In this model a factor $f$ is introduced: $f>1$ represents repulsive forces which increase the free energy of the stable configurations and hence decrease the free energy barrier for diffusion as a function of loading, while attractive forces, $f<1$, stabilize the stable configurations, resulting in an increase of this barrier. Compared to the case $f=0$ (no interactions) for attractive forces, a stronger decrease of the diffusion coefficient is found while for repulsive forces a less strong loading dependence is predicted or even an increase. Figure 35 illustrates the effect of these interactions on a model MFI lattice. By comparison with molecular dynamics simulations of various molecules in $\mathrm{MFI}^{139}$ one can see some of these scenarios. $^{301}$ Whereas the model of Reed and Ehrlich combined with the Maxwell-Stefan formulism can give some insights into how the loading dependence can vary, we have little insight into predicting, even qualitatively, the trend for a given zeolite-guest system.

Saravanan and Auerbach ${ }^{284}$ used a lattice model to study the diffusion of benzene in Na-Y and Na-X (FAU). In their

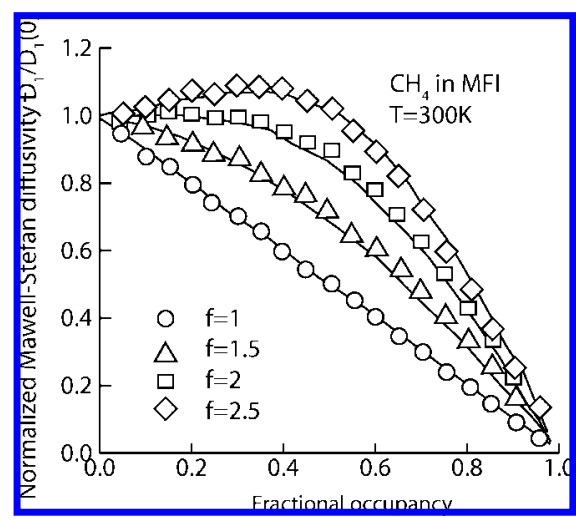

Figure 35. Kinetic Monte Carlo simulation of a MFI-type lattice in which the effect of attractive and repulsive interactions is studied. (Figure based on data from ref 301).

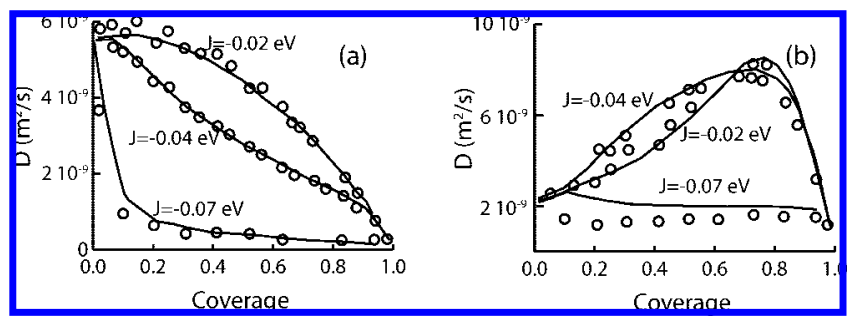

Figure 36. Self-diffusion coefficient of benzene in (a) $\mathrm{Na}-\mathrm{X}$ and (b) Na-Y (FAU) as a function of loading for various adsorbate-adsorbate interactions. Increasing $J$ corresponds to increasing the adsorbate - adsorbate attractions. (Figure based on data from ref 303).

lattice gas model, benzene molecules can occupy a site in the cage or in the window. The interaction energies of particles occupying the lattice sites were fitted to experimental data to reproduce the adsorption thermodynamics. The hopping rates between these sites have been calculated using experimental data for the activation energies and transition state theory ${ }^{303}$ for the Arrhenius prefactors. By varying the adsorbate-adsorbent and adsorbent-adsorbent interaction parameters in the lattice model, Saravanan and Auerbach investigate the effect of changing adsorbate-adsorbate interactions on the loading dependence of the diffusion coefficient. Figure 36 shows that increasing the adsorbateadsorbate interactions has a large effect on the loading dependence of the diffusion coefficient. The increased adsorbate-adsorbate interactions stabilize the stable states and hence decrease the diffusion coefficient of benzene. These figures also indicate that the loading dependence is very sensitive to the cation concentration; for the same adsorbate-adsorbate interaction and zeolite, the diffusion 
coefficient can either increase or decrease with loading depending on the cation concentration.

Other studies that investigate the molecular basis of different types of loading dependencies of the diffusion coefficients can be found in the literature. ${ }^{139,279,283,284,299,303-307}$

\subsubsection{Correlations}

If one makes a mapping of a molecule diffusing in a zeolite onto a random walk on a lattice, one assumes that each jump on the lattice is completely uncorrelated. In the real system this may not be valid on the length scale on which this mapping is made. As a consequence a diffusion coefficient computed from the microscopic hopping rate on the corresponding lattice does not agree with the true diffusion coefficient. It is therefore important to have some understanding of the various correlations. In practice the various forms of correlation effects can occur simultaneously.

Geometry Correlations. The geometry of the zeolite can cause correlations between these diffusion coefficients. For example, in the MFI structure (see Figure 37) diffusion in the $z$-direction can only take place via jumps in the $x$ - and $y$-directions. As a result of these geometry correlations, the diffusion coefficient in the $z$-direction is related to those in the other directions. ${ }^{308}$

$$
\frac{c^{2}}{D_{z}}=\frac{a^{2}}{D_{x}}+\frac{c^{2}}{D_{y}}
$$

For other zeolite structures, different relations hold.

\section{Kinetic Correlations.}

In an ideal random walk it is assumed that after a jump the particle remains sufficiently long in its new location such that it completely loses its memory. If, however, such a complete randomization does not take place and the particle retains a component of the velocity from the previous "jump", the probability of a subsequent jump in this direction will be larger than that in other directions. This form of correlation is called a kinetic correlation. ${ }^{309}$

Suppose the diffusion of a guest molecule can be seen as a sequence of jumps; then the mean square displacement is given by

$$
\begin{gathered}
\left\langle x^{2}(t)\right\rangle=\left\langle\left(\sum_{i=1}^{N(t)} \Delta x_{i}\right)^{2}\right\rangle \\
=\sum_{i=1}^{N(t)}\langle\Delta x\rangle^{2}+2 \sum_{i=1}^{N(t)} \sum_{j=1}^{N(t)-i}\left\langle\Delta x_{i} \Delta x_{i+j}\right\rangle
\end{gathered}
$$

where is the displacement in a single jump. If the jumps are uncorrelated, $\left\langle\Delta x_{i} \Delta x_{i+j}\right\rangle=0$ and we recover the random walk displacement. For correlated jumps, the cross term, however, does not disappear and for the same hopping rate, the diffusion coefficient can be an order of magnitude larger or smaller. $^{309}$

A "trivial" form of kinetic correlations occurs if one uses a lattice in which the lattice sites are not separated by sufficiently high free energy barriers. For example, if one uses a lattice representation in which there are more than one lattice sites inside the cage of zeolite, if a particle arrives at such a site, there is no free energy barrier to hold this particle sufficiently long to randomize the velocity and hence this particle will subsequently continue to move in the direction of its velocity. One may argue whether these types of correlations are not simply a correction for the fact that at the length scale of intracage "hopping" one cannot describe the motion correctly as a random walk.

Kärger et al. ${ }^{310}$ developed a simple two-step model to take these correlations into account. The idea is that if we consider a sequence of two hopping events as an elementary step of the random walk, the correlation between these two-steps is so small that it can be ignored. Kärger et al. used molecular dynamics simulations to compute these two-step hopping rates and showed that if ethane hops from one intersection to another via a zigzag channel, there is a higher probability that the molecule in the next hop reverses its velocity. Therefore ethane has a higher probability to jump back to its original position. An ethane molecule in the straight channel, however, has an equal probability to jump to any of the possible positions. This correlation effect reduces the diffusion coefficient in the direction of the zigzag channels. Similar effects have been found for other diatomic and triatomic molecules ${ }^{311,312}$ while the diffusion coefficient in the direction of the straight channels is enhanced. Of course, in practice two-steps may not be sufficient to eliminate all correlations and one can generalize this approach to higher order steps ${ }^{309,312}$ and other geometries. ${ }^{309}$ Such kinetic correlations have been observed by Jousse et al., ${ }^{313}$ for butane isomers in theta-1 (TON), and by Kärger et al., ${ }^{310}$ for ethane in MFI.

Interestingly, one can use the geometry correlations in MFI to compute the effect of these kinetic correlations. ${ }^{156,314}$ Equation 59 shows that, because of the geometry of MFI, the diffusion coefficient in the $z$-direction is correlated to jumps in the $y$ - and $x$-directions. The derivation of this equation, however, assumes that the jumps do not have any velocity correlations. Hence, by computing

$$
\beta=\frac{\frac{c^{2}}{D_{z}}}{\frac{a^{2}}{D_{x}}+\frac{b^{2}}{D_{y}}}
$$

one can detect a velocity correlation if $\beta \neq 1$. Lopez et al. ${ }^{315}$ studied systematically these correlations for methane at very low loading and observed significant deviations from the $\beta$ $=1$ value, in particular at low temperatures. Further analyses showed that these deviations arise from molecules that continue to jump in the same direction as where they came from. Jousse et al. ${ }^{309}$ developed an analytical approach to take into account both geometry and kinetic correlations.

Vacancy Correlations. A different form of correlations, vacancy correlations-having the opposite effect on the diffusion coefficient-can occur at high loading. Once a molecule jumps to a vacancy, the probability that it will jump back to its original position is much higher than to any other direction simply because this position has by definition a much higher probability to be vacant then any of the other positions. Vacancy correlations have been studied by Saravanan et al. ${ }^{316}$ and Coppens et al. ${ }^{317}$ For a phenomenological description of correlations, within the Maxwell-Stefan framework, see Krishna and van Baten. ${ }^{261,318}$

\subsection{Diffusion of Simple Pure Components}

As we have seen in the previous section, we can find three diffusion coefficients: the self-diffusion, the corrected or collective, and the transport coefficient.

-The self-diffusion coefficient, $D^{\mathrm{s}}$, gives the diffusion of a tagged particle and is obtained from NMR and quasi elastic 


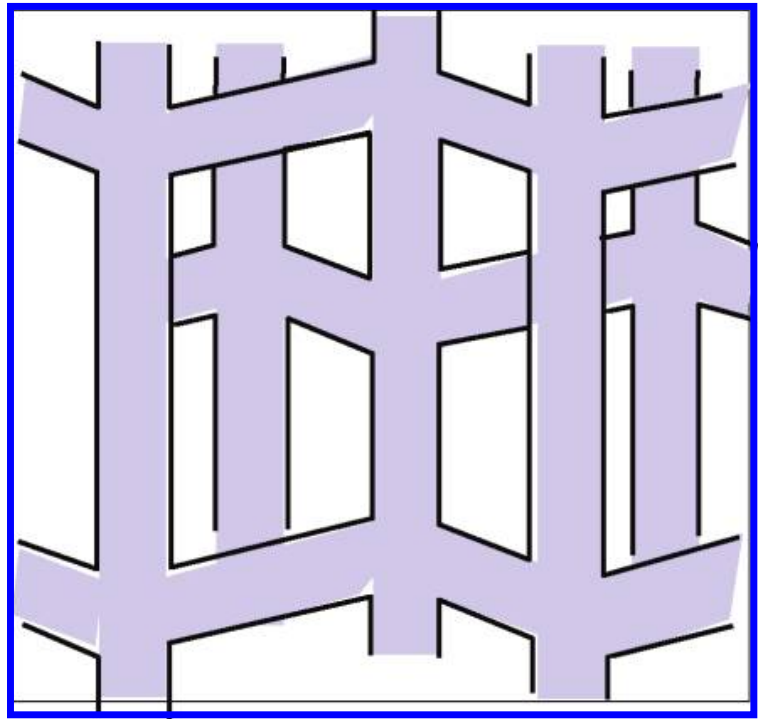

Figure 37. Schematic drawing of the MFI structure consisting of 10-ring channels: straight channels, with an elliptical cross section of $5.5 \times 5.2 \AA^{2}$, and sinusoidal channels, with a nearly circular cross section of $5.4 \AA$. These two channels cross, giving cavities of length $9 \AA$ at the intersection. Silicalite is the all-silica version of the ZSM-5 catalyst, both having the MFI structure.

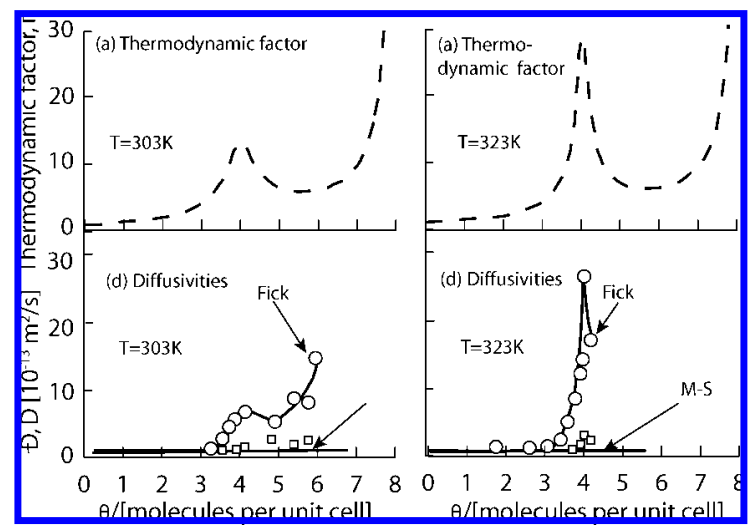

Figure 38. Thermodynamic correction factor $\Gamma$ and diffusivities, Maxwell-Stefan (M-S) and Fick, as a function of loading of benzene in MFI at two different temperatures. The experiments are from ref 323, and the figure is based on data from ref 324.

neutron scattering and can be computed in molecular simulations.

-The transport diffusion coefficient, $D^{\mathrm{t}}$, is the quantity of interest in particle applications, as it determines the efficiency of mass transport. Experimentally, this quantity is measured in macroscopic uptake experiments.

-The collective diffusion coefficient, $D^{\mathrm{c}}$, measures the displacement of the center of mass of the system and can be computed in a molecular dynamics simulation.

In the limit of zero loading, the three diffusion coefficients become identical. If the complete adsorption isotherm is known, one can directly convert the transport diffusion coefficient into a collective diffusion coefficient and vice versa.

It is instructive to look at the diffusion of benzene in MFI to illustrate the points above. Figure 38 shows the thermodynamic factor and the Maxwell-Stefan (M-S) and Fick diffusion coefficients as a function of loading. The thermodynamic correction, which follows directly from the adsorption isotherm, approaches infinity at the maximum loading. Indeed, toward the maximum loading, one needs to increase

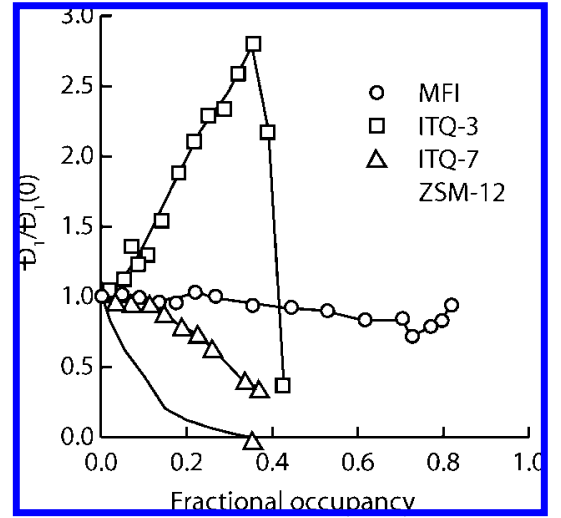

Figure 39. Molecular dynamics simulations of the diffusion coefficient of methane as a function of loading in various zeolites; this figure is based on data from ref 260 .

the chemical potential by an infinite amount to increase the concentration. Hence, eq 36 shows that at these conditions a small concentration gradient gives a very large $\Gamma$. Or, in more physical terms, if a zeolite is saturated, the addition of a molecule at one end of the zeolite causes at the other end of a molecule to leave. For the transport diffusion coefficient, it does not matter which particle leaves the zeolite; this translate to an infinitely large diffusion coefficient. Also at a loading of 4 molecules per unit cell, the thermodynamic diffusion coefficient shows a nonmonotonic behavior. This is related to the fact that benzene prefers to be adsorbed at the intersection and one needs an "extra" pressure to adsorb benzene molecules between intersections. This plateau in the adsorption isotherm is causing the local maximum in the plot of $\Gamma$, and this behavior is reflected in the Fick diffusion coefficient. If one, however, removes the thermodynamic factor using eq 38, we obtain the corrected or Maxwell-Stefan diffusion coefficient. This diffusion coefficient depends much less on the loading.

The above illustrates what is know to be the Darken assumption; that is, the corrected diffusion coefficient is assumed to be independent of the loading. At this point it is important to mention that it was not Darken that made this assumption. In fact, Darken realized that in many cases the corrected diffusion coefficient does depend on loading. For a nice historical note on this, we refer to the article of Reyes et al. ${ }^{319}$ Nevertheless, in many engineering applications this assumption has been widely used. Indeed, from a practical point it is very convenient, as it implies that it is sufficient to have knowledge of either the self-diffusion coefficient at low loading or a single transport diffusion coefficient plus the complete adsorption isotherm to estimate the transport diffusion coefficient for all loadings.

\subsubsection{Loading Dependence}

Although deviations from the Darken assumption have been reported, ${ }^{319}$ these were believed to be the exceptions. Sholl and co-workers ${ }^{139,307}$ used molecular dynamics simulations to systematically investigate the Darken assumption for methane in various zeolites. Figure 39 shows that for most zeolites the Darken assumption does not hold. In fact, depending on the pore topology, the diffusion coefficient can even increase as a function of loading. Similar observations have been made by Krishna and co-workers for other gases ( $\left.\mathrm{He}, \mathrm{Ne}, \mathrm{Ar}, \mathrm{Kr}, \mathrm{H}_{2}, \mathrm{~N}_{2}, \mathrm{CO}_{2}\right) .{ }^{302}$ These very important results of Sholl and co-workers rationalize that the observations made by many other groups, which reported deviations 
of this assumption, might be more the rule than the exception. It is interesting that even for the system for which Figure 39 appears to hold, simulations of Beerdsen et al. ${ }^{320}$ showed a sharp decrease of the diffusion coefficient at higher loadings than have been considered by Sholl and co-workers. These studies clearly show that only for a very few number of systems does the Darken assumption hold.

That the Darken assumption has played such an important role in this field can be explained by the fact that, exactly for those systems for which there is a large number of experimental data, this is a reasonable assumption. That subsequently experimental data have been analyzed assuming this assumption to hold may have contributed to the large scatter in the experimental diffusion coefficients. ${ }^{3,321}$ Clearly, these experiments may need to be reanalyzed. In this respect, it is important to mention that a permeation experiment on zeolitemembranes resulted in loading dependent(Maxwell-Stefan) diffusion coefficients that are in excellent agreement with molecular dynamics simulations. ${ }^{322}$ In addition, from a scientific point of view it is important to obtain a molecular understanding of this loading dependence.

Figure 39 also shows one of the limitations of conventional molecular dynamics; the resulting diffusion coefficients are equally difficult to interpret as the experimental ones. In the following we demonstrate that we can obtain a better understanding if we make a transformation in which we see the diffusion process as hopping on a lattice. Molecular simulations can be used to compute the effective hopping rate directly. This hopping rate follows from a free energy barrier that connects the lattice sites. As we will demonstrate, these free energy barriers are more easy to interpret at the molecular level.

\subsubsection{Methane in MFI}

Also for diffusion, many computational studies focus on alkanes in MFI. ${ }^{47-49,91,141,156,294,315,325-335}$ From a computational point of view, this is an attractive system, since the diffusion coefficients are sufficiently high to be computed using conventional molecular dynamics simulations. An illustration of the rapid increase in CPU power is that in 1992 state of the art supercomputers allowed June et al. ${ }^{47}$ to simulate butane and hexane, while in 1997 Runnebaum and Maginn $^{48}$ could simulate chains as long as $n-\mathrm{C}_{20}$.

At present, many experimental and simulation data have been published, yet it is difficult to open a text on diffusion in zeolites that does not start with a figure similar to Figure 40. This figure sends the message that, depending on the experimental technique, diffusion coefficients are found of linear alkanes in MFI that can vary many orders of magnitude. Indeed, compared to adsorption in MFI, our knowledge on diffusion in MFI is in far less good shape.

The state of the (experimental) art seems to be that one can distinguish microscopic techniques like pulse field gradient NMR and quasi elastic neutron experiments (QENS) that are in reasonably good agreement. ${ }^{336}$ The more macroscopic techniques which are based on measuring changes in the weight of the zeolite can deviate significantly from the microscopic techniques. ${ }^{337}$ Many different possible explanations have been put forward, but we are still lacking a detailed understanding. For example, NMR and neutron experiments study the diffusion of the molecules in the crystals, while gravimetric techniques rely on molecules entering or leaving the zeolite and may therefore also probe surface barriers. ${ }^{337}$ Another important factor is that each of

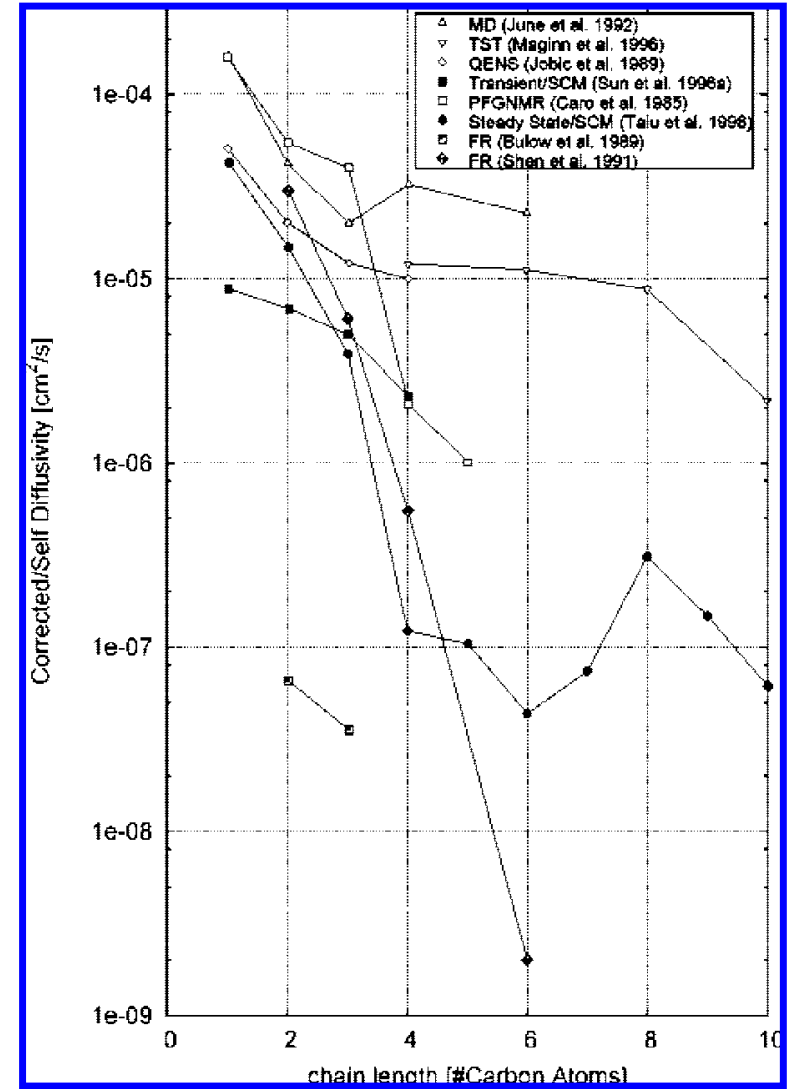

Figure 40. Comparison of the experimental and simulated diffusion coefficients of linear alkanes in MFI as a function of chain length. Figure reproduced with permission from ref 321. Copyright 2006 American Chemical Society.

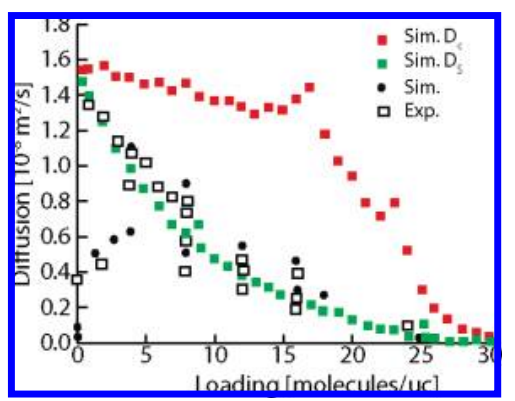

Figure 41. Comparison of experimental and simulated diffusion coefficients of methane MFI as a function of loading. (Figure based on data taken from ref 321).

the experimental techniques probe the diffusion over different length scales, QENS probes motion over nanometers, while NMR measures on the micrometer length scale and might therefore be more sensitive to defects in the zeolite crystals. ${ }^{338}$ The consensus is that molecular simulations are in reasonable agreement with microscopic techniques. ${ }^{243,334,338,339}$

It is interesting to discuss the most simple hydrocarbon, methane, in MFI in more detail. Methane has been simulated by many groups. ${ }^{47,91-93,139,140,150,157,169,264,291,292,294,295,307}$ $315,321,325,327,340-345$ Figure 41 shows the diffusion of methane as a function of loading. The difference between Figures 40 and 41 is that in the former it is implicitly assumed that the diffusion coefficients are independent of the loading. Clearly, this figure indicates a much better agreement of the various experimental and simulation results. Essential to obtain this agreement is that in Figure 41 for each data point the loading has been carefully estimated for each experimental data point. The figure also shows some experimental data points that show 


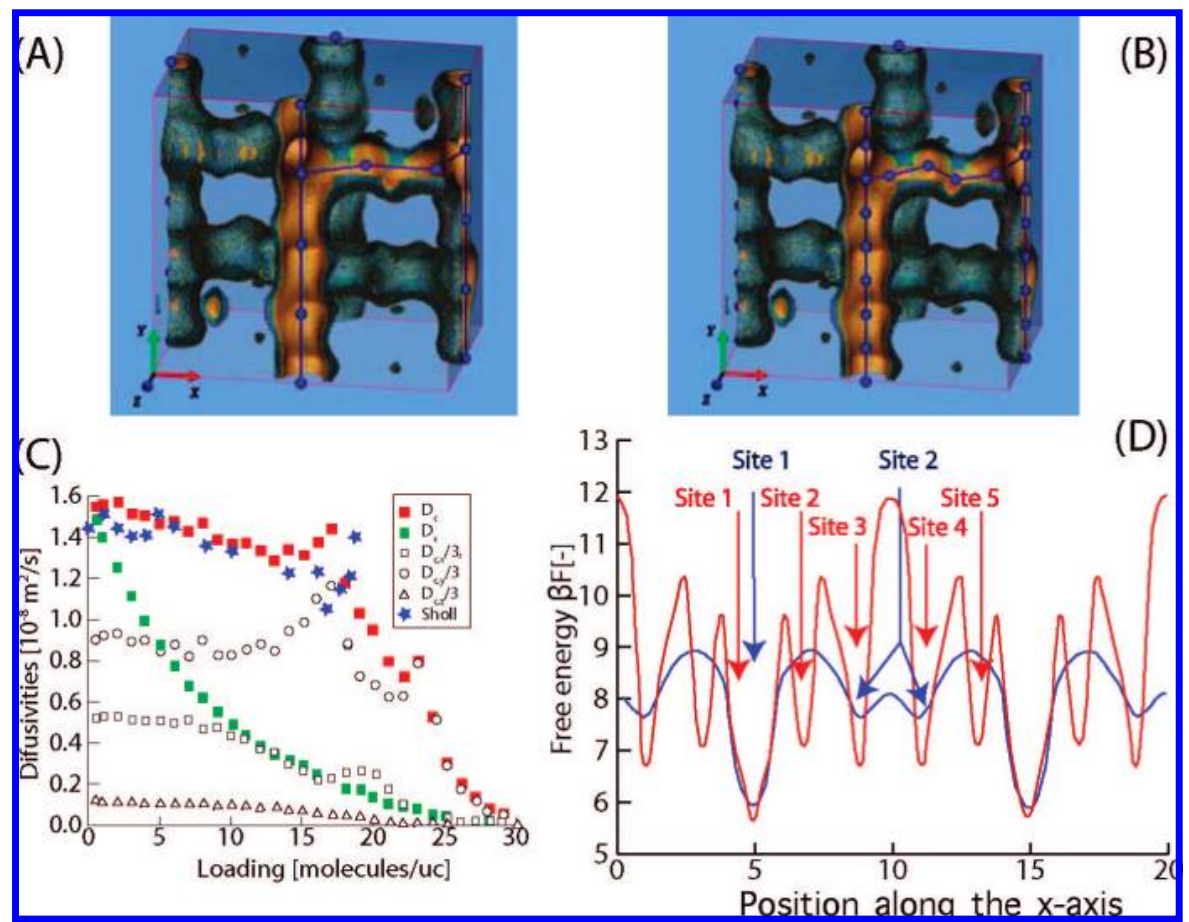

Figure 42. Diffusion of methane in MFI: parts (A) and (B) show the adsorption sites of methane in MFI at low and high loading, respectively, part (C) shows the diffusion coefficients in the various directions, and part (D) shows the free energy profile along the straight channel (y-direction) at various loadings. Parts (A) and (B) are reproduced with permission from ref 320 . Parts (C) and (D) are based on data from refs 3 and 320 .

large differences. This may be caused by a poor estimate of the loading or by the fact that, indeed, macroscopic techniques also for methane show large deviations.

There are a few other interesting points to note in Figure 41. If we make a comparison with Figure 39, one could argue that on the basis of this figure the Darken assumption would hold for methane in MFI. Figure 41, however, shows that, for higher pressure, also for MFI the Darken assumption breaks down. This figure therefore illustrates it is very important to have a detailed understanding of the loading dependence.

The other interesting phenomenon in Figure 41 is the peak in the collective diffusion coefficient at 16 molecules per unit cell; careful inspection of the collective diffusion coefficient shows more local maxima (e.g., loadings of 2, 8, or 24). As the accuracy of the simulation results in Figure 41 is smaller than the symbol size, these "humps" do not disappear if the simulations are extended for a very long time, but this irregular behavior is intrinsic to these systems. ${ }^{320}$ Figure $42 \mathrm{C}$ shows that each hump can be associated with a corresponding hump in only one of the three components of the diffusion coefficients. Figure 42D shows the loading dependence of the free energy profile of a methane molecule moving along the straight channel $(y$ direction). At low loading there are three adsorption sites: two in each of the intersections and one in the middle of the channel (the figure shows two minima, but only one can be occupied). These adsorption sites are visualized in Figure $42 \mathrm{~A}$. If one further increases the loading to the point that all the low loading adsorption sites are occupied, the system needs to create "space" for the additional molecules. The free energy profiles show additional adsorption sites at high loading (see Figure 42B). One can visualize this as the fact that at low loading the molecules hop on a lattice that suddenly "changes" as the loading is increased. As the number of lattice sites has changed, the loading dependence will have a different slope. In addition, at the point the system "switches" from one lattice to the other, the free energy profile becomes relatively flat and hence causes an increase of the diffusion coefficient. As such changes depend on the details of the channel, one can understand that a similar effect occurs in the zigzag channel at a different loading.

It is interesting to compare these "humps" in the collective diffusion coefficient as a function of loading with the observation of Krishna and co-workers. ${ }^{302,306,346-350}$ Krishna and co-workers used kinetic Monte Carlo and molecular dynamics simulations to demonstrate that if the thermodynamic factor $\Gamma$ (see eq 36) as a function of loading exhibits a strong inflection, this is reflected in a corresponding inflection in the Maxwell-Stefan or collective diffusion coefficient versus loading. ${ }^{302,306,346-348}$ Experimental confirmation for the anticipated inflection behavior is due to QENS measurements of Jobic et al ${ }^{349}$ for $n$-hexane and $n$-heptane in MFI. Chmelik et al. ${ }^{350}$ performed infrared microscopy experiments for diffusion of isobutane in MFI zeolite to show that the inflection in the Maxwell-Stefan diffusivity can be captured very well using KMC simulations and is in agreement with simulations that were published earlier. ${ }^{306}$ Beerdsen et al. ${ }^{3}$ show that changes in the adsorption at the molecular level can have a large effect on the loading dependence of the diffusion coefficient. Some of these changes in the adsorption behavior can also induce inflections in the adsorption isotherm, which gives a molecular explanation of the correlation found by Krishna and coworkers between inflections in the adsorption isotherms and changes in the loading dependence of the collective or Maxwell-Sefan diffusion coefficient.

Lopez et al. ${ }^{315}$ used molecular dynamics simulation to study the temperature dependence of the diffusion at the low loading limit of methane in MFI. Lopez et al. observed at low temperature a non-Arrhenius behavior (see Figure 43). To explain the non-Arhhenius behavior, Lopez et al. 


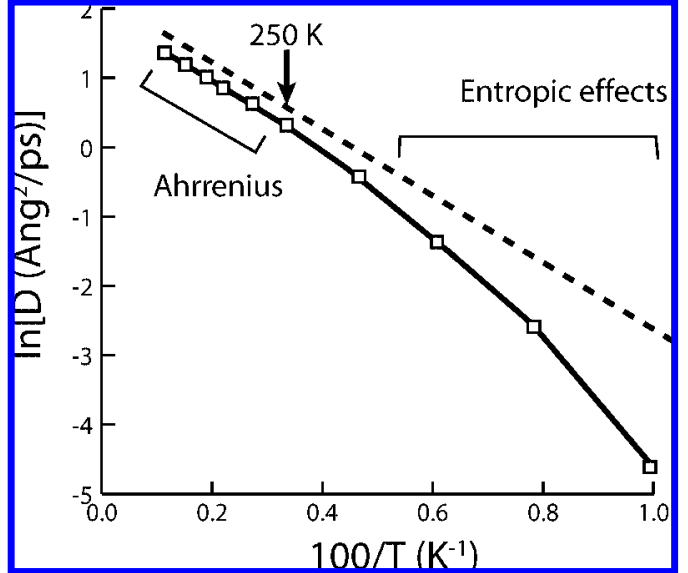

Figure 43. Diffusion coefficient of methane in MFI as a function of temperature. Crossover to non-Arrhenius-type behavior below $T=250 \mathrm{~K}$. (Figure based on data from ref 315).

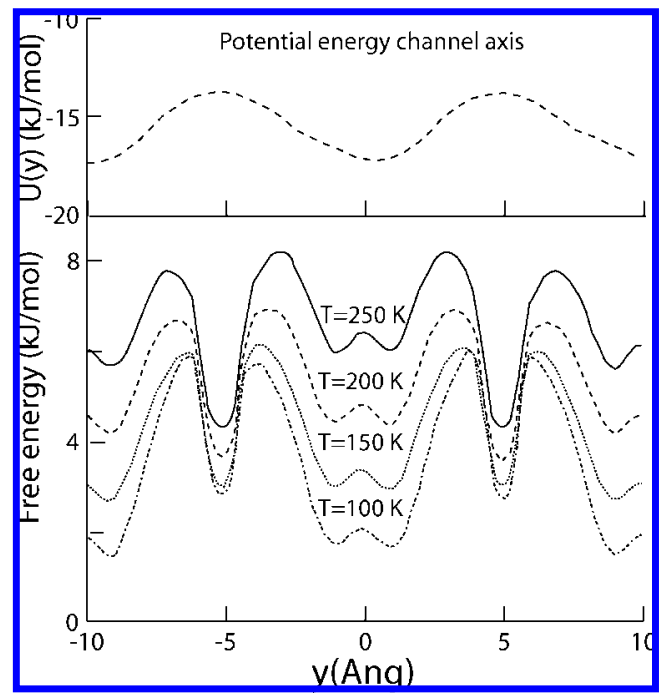

Figure 44. Energy (top figure) and free energy (bottom figure) of a methane molecule as a function of the position along the straight channel in MFI for various temperatures. (Figure based on data from ref 315).

computed the free energy barriers for a methane molecule to jump from intersection to intersection. Figure 44 shows this profile for various temperatures. At low temperatures the lowest free energy corresponds to a position in the channel while above $T=250 \mathrm{~K}$ the lowest free energy is at the intersection (i.e., the zigzag channels that project on the intersection). With these free energy profiles one can understand the non-Arrhenius behavior; at low temperature the main diffusion barrier is a particle leaving its preferred adsorption site in the middle of the channel, while at high temperature the main barrier is a particle leaving the intersection. As these barriers are different, one can understand the difference in slope in Figure 43.

The lack of accurate and consistent experimental data as is suggested in Figure 40 makes it very difficult to validate the force field and the assumptions underlying a given model. The fact that in Figure 40 all results are plotted in the same figure, irrespective of the loading, illustrates that for a very long time it was assumed that diffusion results in MFI, and many other structures are independent of the loading. For methane, however, we have shown that a careful analysis of the experimental data illustrates that part of the incon-

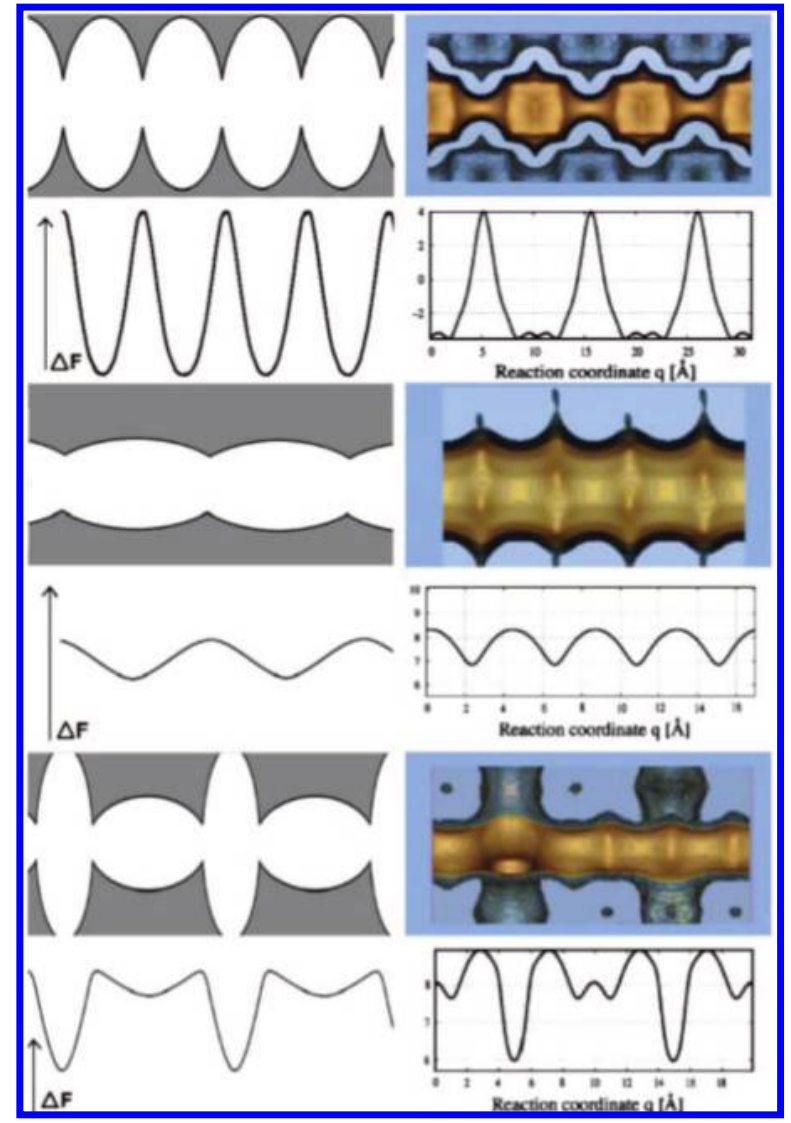

Figure 45. Zeolites made out of ellipsoids. The left figures show three ways in which these ellipsoids can be connected, and below the corresponding free energy profile of a molecule diffusing through these structures. The right figures give an example of real zeolites (top, SAS; middle, AFI; bottom, MFI) and methane diffusing through them at zero loading. Figure reprinted with permission from ref 351. Copyright 2006 The American Physical Society.

sistencies can be attributed to this assumption. It would be interesting to perform a similar analysis for the other systems.

\subsubsection{Methane in Other Zeolites}

The results of Skoulidas and Sholl shown in Figure 39 indicate that, depending on the pore topology, the corrected diffusion coefficient can either increase, decrease, or remain constant if one slightly increases the loading. In particular, the fact that the diffusion coefficient increases when we increase the loading is surprising; it is difficult to explain to people that one should increase the number of cars on the road to increase the mobility.

Beerdsen et al. ${ }^{3,351}$ assumed that diffusion can be described by a hopping process from one site to another on a lattice model of the zeolite. For a given molecule, Beerdsen et al. computed the free energy profile of a molecule hopping from one site to another. These free energy profiles allow us to make a classification that helps us understand the loading dependence. This classification is shown in Figure 45. The assumption is that one can model zeolite as ellipsoids. One can make one-dimensional zeolites by placing the ellipsoids on a line and connecting them. The circle connecting two ellipsoids defines the window diameter, and depending on the orientation of the ellipsoids one gets one-dimensional tubes (Figure 45b) or a zeolite with a more cagelike character (Figure 45). By connecting the ellipsoids in alternating orientations, one can mimic a two- (or three-) dimensional 


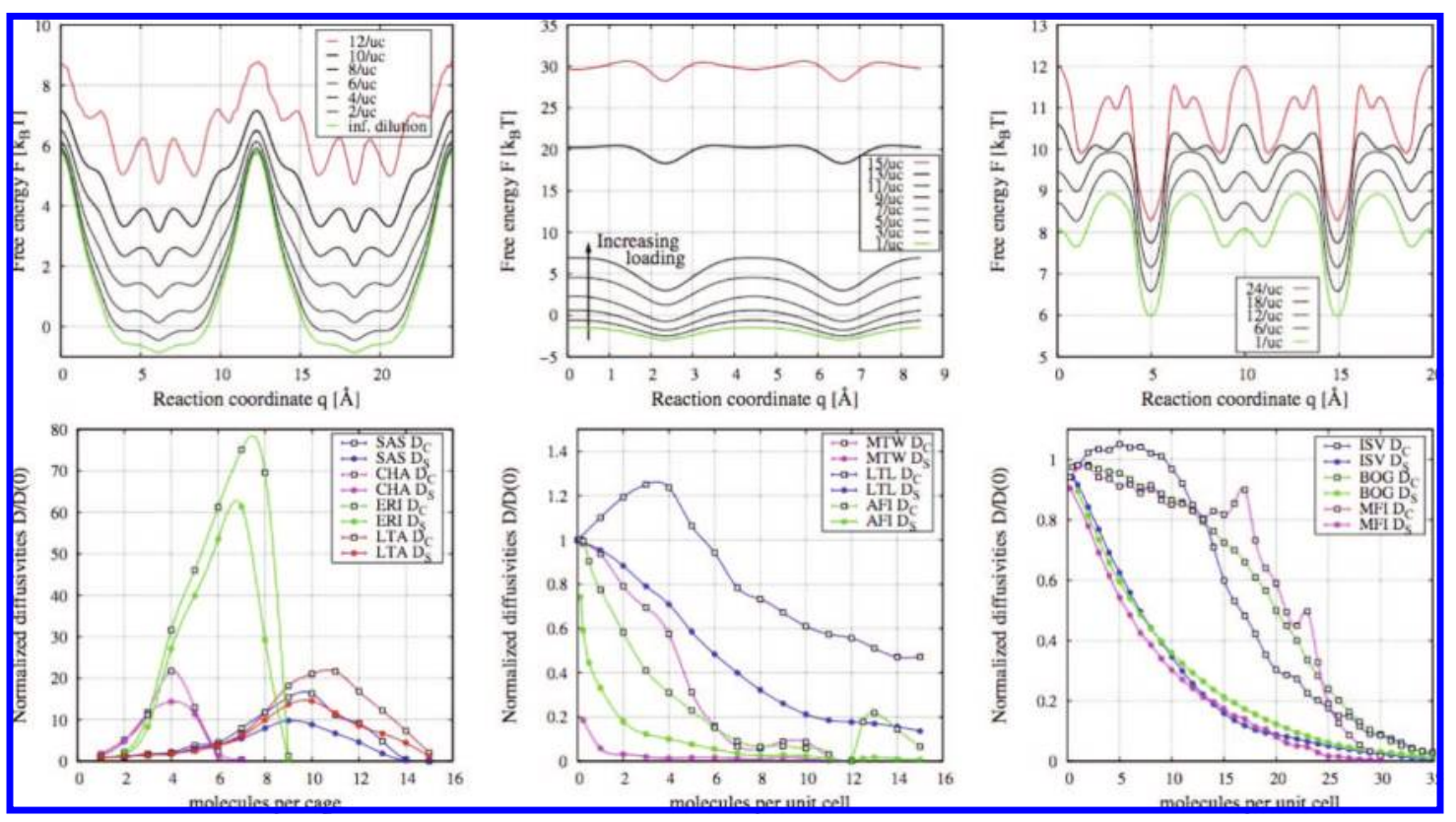

Figure 46. (Top) Free energy profiles and diffusion coefficient of methane in LTA (left), AFI (middle), and MFI (right) and (bottom) normalized self- and collective diffusion coefficient as a function of loading for the cagelike zeolites SAS, CHA, ERI, and LTA (left), the tubelike zeolites MTW, LTL, and AFI (middle), and the intersecting channels BOG, MFI, and ISV (right). Figure reprinted with permission from ref 351. Copyright 2006 American Chemical Society.

structure. The corresponding free energy profiles below the schematic show that the barrier for diffusion in the case of a tube is very small if the ratio of the window diameter and the diameter of the middle part of the ellipsoid is close to one. A very different situation is the cagelike structure in which the windows form a barrier for the diffusion, while for the 2-dimensional structures the vertically oriented ellipsoids form entropic traps. The right part of the figure shows some examples of real zeolite structures and the corresponding free energy profile of methane.

We now consider the effect of increasing the loading; as all ellipsoids are identical for all structures, the "second" molecule will be preferentially placed at the same location in the ellipsoid. In fact, to a first approximation, this probability is the highest where the free energy in Figure 45 is the lowest. At this point it is important to mention that the hopping rate and hence the diffusion coefficient are not only determined by the free energy barrier but are the product of this free energy barrier and the recrossing coefficient (see section 3.2). However, for all systems that have been studied, this coefficient is a monotonically decreasing function of the loading. We can now envision the following scenarios:

-Tubelike zeolites: there is little preference for the additional molecules to be adsorbed. However, as the molecules prefer to be in contact with the walls rather than to be in contact with another molecule, as a consequence, the free energy profile shifts to higher values but there will be little difference in the shift of the top of the barrier and the bottom. To a first approximation, one would expect the free energy barrier to remain constant, and hence, the recrossing coefficient causes a decrease of the diffusion coefficient as a function of loading.

- Cagelike zeolites: for these zeolites the preferential adsorption is in the cages. Hence, additional molecules will increase the bottom of the free energy profile but not the top. Hence, additional molecules will lower the free energy barrier and one would expect an increase of the diffusion coefficient.
-Two- or three-dimensional structures: here the adsorption will be in both the horizontal and vertically oriented ellipsoids. In the horizontal ellipsoids, they will form an additional barrier, and in these, additional molecules will make the vertically oriented ellipsoids less attractive. As the latter effect will be smaller, the net result is an increase of the free energy barrier, resulting in a decrease of the diffusion coefficient.

Figure 46 gives some examples of diffusion of methane in zeolites with the topologies shown in Figure 45.

The above free energy arguments only apply for the selfdiffusion coefficient. To understand the collective diffusion coefficient, one has to take into account the lattice topology and other factors that influence the collective behavior.

\subsection{Diffusion of Long Chain Hydrocarbons}

\subsubsection{Hydrocarbons in MFI}

Also for the longer hydrocarbons in MFI a relatively large number of studies have been published ${ }^{164}$ (e.g, ethane, propane, butane, ${ }^{295,334,352}$ pentane, hexane, ${ }^{295,334,349,352}$ heptane, ${ }^{349}$ octane, ${ }^{295,328,334,352}$ and longer hydrocarbons ${ }^{338}$ ).

Jobic and Theodorou ${ }^{257,338}$ carefully reviewed the experimental literature, and their conclusions are summarized in Figure 47. This figure shows a reasonable agreement of the experimental data with the results of molecular simulations. Interestingly, Figure 47 shows the QENS data for both NaZSM-5 and silicalite (square with dot) illustrate that the cations slow down the diffusion of the alkanes. Another important point to mention is that the simulations are carried out at zero loading while the experiments are at twelve carbon atoms per unit cell.

Figure 41 shows that for methane the diffusion coefficient at twelve molecules per unit cell is a factor of two lower compared to the value at zero loading. As for the longer hydrocarbons twelve molecules is increasingly closer to the maximum loading, the differences between the zero loading results and the experimental data are expected to increase. 


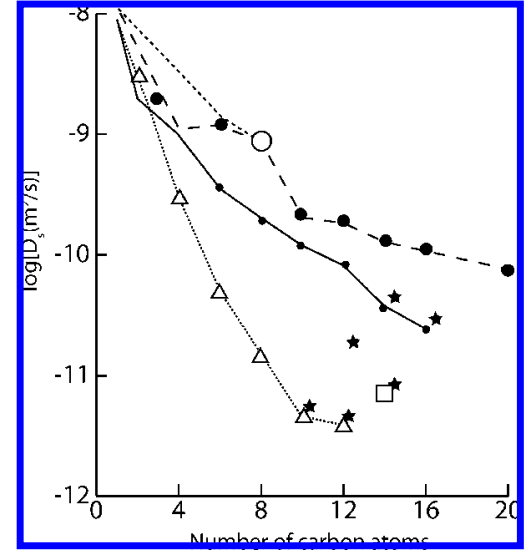

Figure 47. Self-diffusion coefficients of the $n$-alkanes in MFI as a function of chain length at $300 \mathrm{~K}$ as obtained by various techniques: $\bullet$ molecular simulations; ${ }^{156,295} \square$, QENS; $;^{354,338} \triangle$, PFG-NMR. ${ }^{336}$ The asterisks correspond to extrapolations to 300 K. (Figure based on data from ref 338).

Indeed, Chong et al. ${ }^{353}$ made a detailed study of the loading dependence of the diffusion coefficient of ethane in MFI using both QENS and molecular dynamics. The results of Chong et al. ${ }^{353}$ showed both for the transport as well for the self-diffusion coefficient excellent agreement between the two techniques. As the force field ${ }^{94,97}$ used by Chong et al. was fitted to adsorption data only, it is not obvious that this force field would also predict a correct diffusion behavior, and therefore the agreement found by Chong et al. is very encouraging. For a loading of six molecules (=twelve carbon atoms) per unit cell, the diffusion coefficient is a factor of three lower compared to the value at zero loading.

Jobic et al. ${ }^{349}$ made a detailed comparison of the loading dependence of the transport, collective, and self-diffusion coefficients for $n$-hexane and $n$-heptane between molecular dynamics simulations and QENS. Both in the QENS and MD results, the transport diffusion coefficient of heptane shows a sharp increase at four molecules per unit cell. This increase is a signature of the step in the adsorption isotherm for heptane at four molecules per unit cell (see section 5.3.1 and Figure 17), which gives a nonmonotonic thermodynamic factor in eq 38. Interestingly, if we remove the thermodynamic factor from the transport diffusion coefficients, one obtains a collective (or Maxwell-Stefan) diffusion coefficient that shows less dependence on loading but still has an anomalous behavior at four molecules per unit cell. The MD and QENS results are in excellent qualitative agreement, but quantitatively the MD results give a diffusion coefficient that is a factor of five higher compared to QENS.

Figure 47 shows that the molecular simulations predict a nonmonotonic behavior for the diffusion coefficients for alkane chain lengths of 4-6 in MFI. The short-chain alkanes reside in the straight or zigzag channels, and the intersection is the barrier for diffusion. Octane and longer alkanes always have a part residing in the intersection, but to optimally fit in the pores, these molecules need to have conformational defects and the barrier for diffusion is this conformational defect that needs to slide along the chain. ${ }^{156,257,338}$ Raj et al. ${ }^{328}$ argue that this effect leads to a different temperature dependence of the diffusion coefficient of octane compared to the shorter alkanes. Indeed, the computed activation energies $^{257,338}$ for the diffusion reflect this behavior. Figure 47 also shows that not all simulation results predict this behavior. In particular, the molecular dynamics simulations of Leroy et al. ${ }^{295}$ did not give any evidence for this

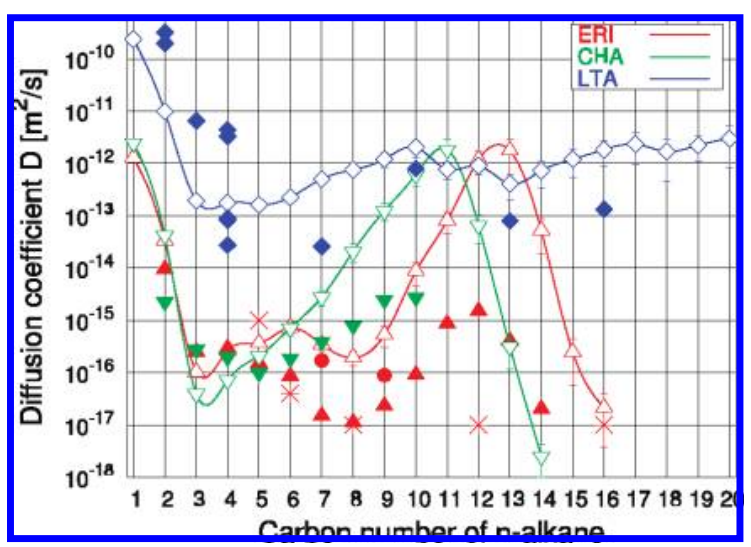

Figure 48. Diffusion coefficients in ERI, CHA, and LTA as a function of chain length at $T=605 \mathrm{~K}$; the simulated diffusion coefficients are given as open symbols, ${ }^{194,192}$ and the experimental data are given by closed symbols. ${ }^{357,358,360,361}$ The data for ERI and the data for LTA (blue) are compiled by Kärger and Ruthven. ${ }^{252}$ The data for $\mathrm{CHA}$ are relative diffusivities. To make a comparison possible, the data have been scaled by assuming that for $n-\mathrm{C}_{3}$ the experimental diffusion coefficients in $\mathrm{CHA}$ are equal to the experimental values. This scaling is used for all other chain lengths. The data for LTA have been extrapolated to $605 \mathrm{~K}$ using the activation energies. ${ }^{252}$ It is important to note that the scatter in the data for butane is caused by the fact that data have been reported by several groups, while for the other alkanes only one data point has been reported. The line connecting the simulation data is a guide to the eye. Error bars on the simulation data are only shown if they are bigger than the symbol size. Figure reprinted with permission from ref 194. Copyright 2003 The American Physical Society.

nonmonotonic behavior. The molecular dynamics simulations of Runnebaum et al. ${ }^{48}$ and Nagumo et al., ${ }^{164}$ on the other hand, do show evidence of this nonmonotonic behavior. Nagumo et al. showed that the diffusion along the zigzag channels decreases monotonically with increasing chain length, while for the straight channels the diffusion coefficient increases as a function of chain length.

Other Zeolites. Simulation of diffusion coefficients is by no means limited to hydrocarbons in MFI. In fact, since far less experimental data on diffusion in other zeolites is available, simulations of other systems are often the only source of information. ${ }^{18,329-331,348,355,356}$ If the size of the molecules approaches the size of the cages or channels of the zeolite, many interesting phenomena can occur. In this section we discuss a few of them.

\subsubsection{Resonance Diffusion}

In most zeolites the diffusion coefficient of the $n$-alkanes decreases monotonically as a function of the chain length. ${ }^{252}$ This seems to be an obvious result; the diffusion coefficient of bulk alkanes decreases monotonically with chain length and the longer the chain length, the higher the barriers for the diffusion, since more atoms have to cross. It was therefore very surprising that the experimental data of Gorring ${ }^{357}$ in 1973 showed an increase of the diffusion coefficient as a function of the chain length of the $n$-alkanes in zeolite T, which is an intergrowth of ERI and OFF. A similar effect was observed in Chabazite (CHA) ${ }^{358}$ In Figure 48 these experimental data are shown. This observation is often referred to as the Window effect-a region for which the diffusion coefficients are much higher compared to those for molecules that are shorter or longer. The practical importance of these results is that they suggest that diffusion limitation 


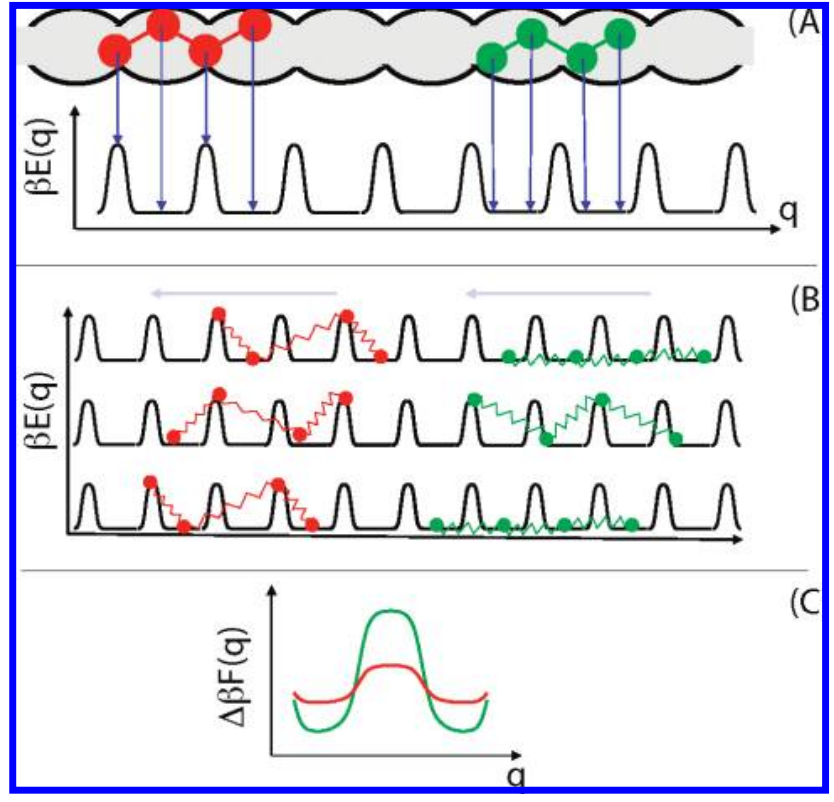

Figure 49. Frenkel-Kontorova model of diffusion in a zeolite: (A) The green molecule is commensurate with the zeolite structure, and the red molecule is incommensurate. (B) We can assign a (free) energy to each atom of the molecule and a spring that accounts for the intramolecular interactions and compute the change of the (free) energy if we displace the molecule. (C) If we add all interactions, we observe that the commensurate molecule has a higher barrier for diffusion compared to that of an incommensurate one.

is the explanation of the bimodal product distribution obtained for the cracking of Erionite (ERI) by Chen et al. ${ }^{359}$ (see section 7.3.4).

Several theories have been published to explain the increase of the diffusion coefficient as a function of chain length. ${ }^{362-368}$ These theories are related to the FrenkelKontorova model of atoms adsorbed on a substrate. This model predicts that if the molecules adsorb in such a way that it is commensurate with the substrate structure, the diffusion coefficient is low, and if the adsorption is incommensurate with the substrate structure, the diffusion coefficient is high. This mechanism is illustrated in Figure 49; if the adsorption is commensurate for the molecule to diffuse, all atoms have to leave their favorite location, resulting in a high energy barrier. If we have an adsorption incommensurate with the substrate, some atoms are at a high energy position and other atoms are at low energy positions; changing the position of the molecules therefore does not change the energy very much. One can have various realizations of this commensurate-incommensurate adsorption. For example, by increasing the chain length, one can change into a situation in which the adsorption changes from commensurate to incommensurate or vice versa.

Experimentally, it is unclear whether such a window effect exists. The data of Gorring have been cited often; the reliability of these experiments have been questioned ${ }^{128,369}$ (see ref 370 for a detailed discussion on the experimental issues related to these data). In fact, data from the groups of Ruthven $^{360}$ and Conner ${ }^{361}$ did not give evidence for an increase of the diffusion coefficient as a function of chain length. In addition, Ruthven argued using theoretical arguments based on similar ideas to the model of Ruckenstein and Lee that for the zeolite LTA a signature of the Window effect should occur in the $\mathrm{C}_{13}-\mathrm{C}_{16}$ range. ${ }^{360}$ Experimental data for LTA, however, using uptake experiments did not show a window effect ${ }^{371}$ while neutron spin echo experi- ments did show an increase of the diffusion coefficient in the $\mathrm{C}_{6}-\mathrm{C}_{10}$ range. ${ }^{372,373} \mathrm{On}$ a different system, LTL and MTW (ZSM-12), Yoo et al. ${ }^{374}$ observed an increase of the diffusion coefficient upon increasing the chain length.

Ruckenstein and Lee ${ }^{362}$ introduced a one-dimensional model of a rigid molecule in a periodic potential. If the chain length of this molecule is exactly an integer multiple of the period of the potential, the energy of the molecule does not depend on diffusion and hence there is no diffusion barrier. If the ratio of the length of the molecule and the periodicity is integer, the diffusion takes its maximum value. Ruckenstein and Lee introduced the term resonance diffusion to indicate that an incommensurate molecule has an unusually high diffusion coefficient. Application of this theory for the $n$-alkanes in ERI using a very simple model of ERI and alkane molecules indicates maxima of the diffusion coefficients for $\mathrm{C}_{4}$ and $\mathrm{C}_{10}$ and a minimum for $\mathrm{C}_{7}$. The theory of Ruckenstein and Lee focuses on energy barriers. To compute a diffusion coefficient, this theory was extended to include the dynamics of a particle moving in this energy landscape using Langevin dynamics ${ }^{365,366}$ or Brownian dynamics. ${ }^{364}$ Although these theories nicely illustrate that a molecule moving in a periodic potential with barriers can result in an increase of the diffusion coefficient as a function of chain length, the theories are quasi-phenomenological, which makes it difficult to make a direct comparison with the experimental data. The zeolites ERI, CHA, and LTA were studied by Dubbeldam et al. ${ }^{194}$ using rare event simulations. Figure 48 shows that these simulations are in very good agreement with the experimental data, if one takes the scatter in the experimental data into account. The agreement is remarkable if one realizes that the models have been optimized for the adsorption of alkanes in MFI. The simulations also demonstrate that for LTA an increase of the diffusion coefficient should be expected for $\mathrm{C}_{24}$ and longer. For the shorter chains one can also expect nonmonotonic behavior, but this behavior is related to the intracage barrier. ${ }^{192}$ It is tempting to conclude that this nonmonotonic behavior has been observed experimentally, ${ }^{254,372}$ but as the experiments are done on zeolites with cations, it is important that simulations are carried out on exactly the system that is studied experimentally.

Runnebaum and Maginn ${ }^{48}$ have used molecular dynamics simulations to study resonant diffusion. With molecular dynamics it is not possible to access the very low diffusion in ERI or CHA. In analogy to the theory of Ruckenstein and Lee, Runnebaum and Maginn argued that also the linear alkanes in MFI feel a periodic potential, and therefore one can define a resonance chain length. Their simulations showed that the effects in MFI are too small to be observed in the overall diffusion coefficient. But for the diffusion coefficient in the direction of the straight channels [010], an increase in the diffusion coefficient in the ranges $\mathrm{C}_{5}-\mathrm{C}_{9}$ and $\mathrm{C}_{14}-\mathrm{C}_{16}$ was observed. Talu et al. ${ }^{253}$ confirmed these observations experimentally using single-crystal membrane experiments.

Various other manifestations of resonance diffusion have been observed. Yashonath and co-workers ${ }^{375-379}$ observed in MD simulations of noble gases in zeolite A (LTA) and Y (FAU) an increase of the diffusion coefficient as a function of the diameter of the noble gas. This effect was denoted the levitation effect. Similar effects were observed for linear molecules in FAU as a function of the length of the molecule ${ }^{376,380}$ and for pentane isomers in $\mathrm{AlPO}_{4}-5$ (AFI). ${ }^{375}$ 
Both zeolites have cages that are connected via windows which are barriers for diffusion. Here the mechanism appears that if the diameter of the molecule increases beyond a certain value, the energy on top of the barrier increases less than the free energy in the cage. The diffusion barrier, which is the difference between the free energy of a molecule in the cage and in the window, decreases, resulting in an increase of the diffusion coefficient.

Schenk et al. ${ }^{381}$ computed the free energy profiles of $n, m$ dimethylpentadecane isomers in TON depending on the position of the methyl groups; the adsorbate was found incommensurate with the TON structure, and the diffusion barrier became very small. A similar observation was made by Bhide and Yashonath ${ }^{375}$ for pentane and isopentane in AFI.

\subsubsection{Single-File Diffusion}

An interesting case of geometry correlations is onedimensional channels in which the molecules cannot pass each other. In such a system, one does not observe diffusive behavior but single-file diffusion. Single-file diffusion can be of importance for our understanding of catalysis in onedimensional zeolites or for the use of hydrocarbon traps for automobile applications. ${ }^{382}$

In normal diffusion the mean-squared displacement is linear in time (see eq 52):

$$
D=\frac{1}{2} \lim _{x \rightarrow \infty} \frac{\left\langle[z(t)-z(0)]^{2}\right\rangle}{t}
$$

In the case of single-file diffusion, the displacement of a tagged particle depends on its neighbors. This correlated motion results in a mean-squared displacement which is proportional to the square root of the time $\mathrm{e}^{383}$

$$
F=\frac{1}{2} \lim _{x \rightarrow \infty} \frac{\left\langle[z(t)-z(0)]^{2}\right\rangle}{\sqrt{t}}
$$

where $F$ is the single-file mobility. Although single-file diffusion was known theoretically for many years, it was much later confirmed experimentally in zeolites. ${ }^{384-387}$

To investigate single-file diffusion experimentally, one has to use a zeolite with one-dimensional channels that are sufficiently narrow such that the probability that two molecules can pass each other is very small. In addition, the zeolite channels have a finite length, and if molecules leave or enter the zeolite, the correlation that is responsible for single-file diffusion is destroyed. These effects make it very difficult to determine single-file diffusion in experiments. ${ }^{388}$ For example, for methane and ethane in $\mathrm{AFI}\left(\mathrm{AlPO}_{4}-5\right)$, some groups observe single-file diffusion ${ }^{384,385}$ while others conclude normal diffusion. ${ }^{387}$ Simulations have been used to investigate these controversies in detail. Since the results of these studies have not yet converged, a full understanding of this intriguing phenomenon is still lacking.

Adsorption studies show that, at high loading, methane and ethane molecules adsorb in AFI in a helical structure, ${ }^{199}$ and depending on the temperature, these molecules can pass each other. ${ }^{75,389}$ Therefore, depending on the differences between the time scales of these passing events and the time scale of the diffusion experiment, normal or single-file diffusion may be observed. ${ }^{388,390}$ Suppose that the typical time between two passing events is given by $\tau_{\text {pass }}$, for times less than this passing time, single-file diffusion will be observed. For times longer than $\tau_{\text {pass }}$, the tagged particle will pass another particle and on these time scales perform a random walk in which $1 / \tau_{\text {pass }}$ is the hopping rate. As a consequence, at sufficiently long time scales, normal diffusion is observed with a diffusion coefficient related to this hopping rate (see section kinetic $\mathrm{mc}$ ):

$$
\frac{D_{z z}}{t}=\frac{a^{2}}{\tau_{\text {pass }}} \propto \frac{1}{\tau_{\text {pass }}}
$$

where $a$ is the average distance between two particles. In the derivation of eq 62 , it is assumed that the average distance $a$ is independent of the hopping rate. This is a reasonable assumption if $\tau_{\text {pass }}$ is small compared to the time it takes the groups of particles involved in the passing event to cover the distance $a$. Mon and Percus ${ }^{391}$ argue that such small hopping times are only likely for those systems in which the molecules can easily pass each other. Mon and Percus assumed that a tagged particle in a time interval $\tau_{\text {pass }}$ has single-file diffusion and its mean square displacement is given by eq 61, which gives for the average displacement between two passing events

$$
a^{2} \approx\left\langle\left[z\left(\tau_{\text {pass }}\right)-z(0)\right]^{2}\right\rangle=F \sqrt{\tau_{\text {pass }}}
$$

giving for the diffusion coefficient ${ }^{391}$

$$
\frac{D_{z z}}{t}=\frac{F \sqrt{\tau_{\text {pass }}}}{\tau_{\text {pass }}} \propto \frac{1}{\sqrt{\tau_{\text {pass }}}}
$$

Mon and Percus ${ }^{391}$ confirm the predictions of eq 64 via stochastic hard-sphere simulations. Passing events were also observed in molecular dynamics simulations of methane and ethane in AFI. ${ }^{75}$ Sastre and Corma ${ }^{332}$ studied single-file diffusion of benzene and $n$-butane in IFR (ITQ-4) and LTL (zeolite L). In IFR the molecules cannot pass each other, which resulted in single-file diffusion, while in LTL the molecules can pass, resulting in normal diffusion. Sholl and Fichthorn ${ }^{390}$ investigated the interesting case for binary mixtures in which molecules of one of the components can pass each other while molecules of the other component cannot.

In practice, the length of the tubes is finite, and on the time scale of the experiments, a fraction of the molecules will leave or enter the pores. If a molecule leaves the zeolite channel, a vacancy is introduced in the channel and this vacancy can move in the channel. If the number of vacancies is small, we can assume that this vacancy is performing a random walk with a (normal) diffusion coefficient given by $D^{\nu}$. For a finite channel, the displacement of a tagged particle can be seen as the result of a sequence of hopping events that takes the vacancy from one end of the channel to the other end (see Figure 50). The consequence of this observation is that also the tagged particle is performing a random walk with an elementary hopping rate that is related to the time it takes a vacancy to cross the channel. This analysis predicts, for times longer than the time it takes vacancies to cross a channel, normal diffusion behavior for the tagged particles. The diffusion coefficient of this tagged particle will be inversely proportional to the channel length $L:^{388,392}$

$$
D \propto \frac{1}{L}
$$

Single-file diffusion will be observed for times shorter than the time it takes vacancies to cross a channel. Nelson and Auerbach used kinetic Monte Carlo simulations to confirm 


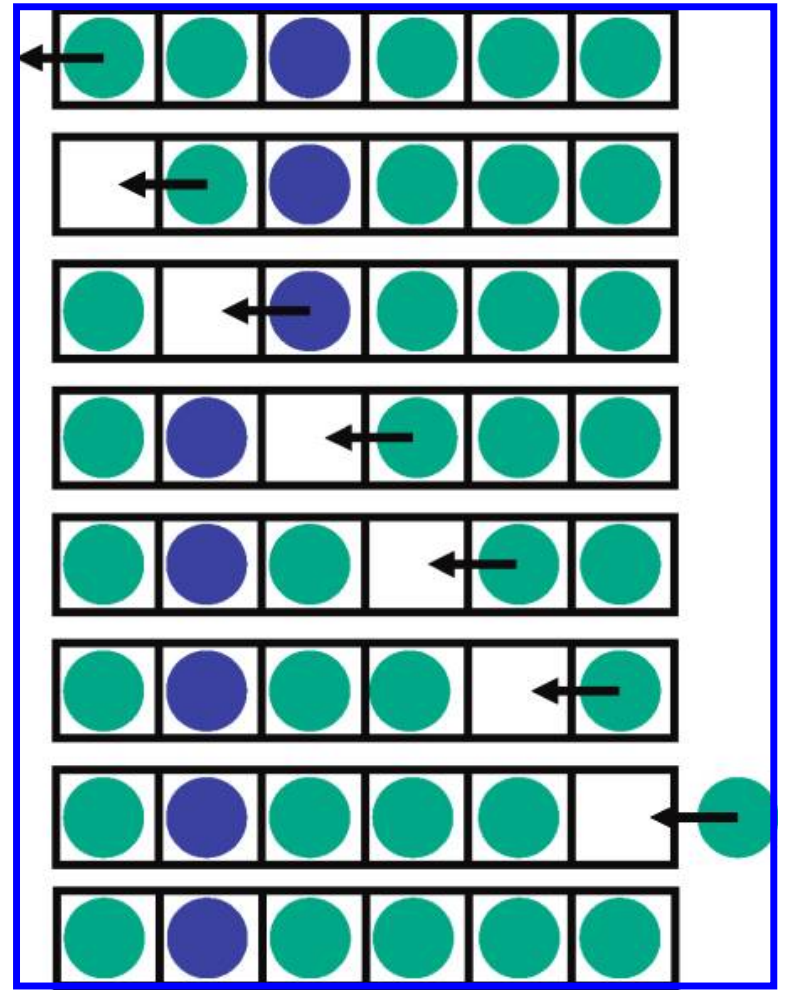

Figure 50. The role of vacancies in single-file diffusion; the displacement of a tagged particle (blue) can be seen as the result of a sequence of hopping events of a vacancy.

these predictions and derived an analytical expression for the diffusion coefficient in such systems. ${ }^{392}$ MacElroy and Suh ${ }^{393,394}$ however, observed in their simulations that the diffusion coefficient scales as $1 / \sqrt{ } L$.

In analyzing the experimental data it is usually assumed that molecules move individually. Sholl and co-workers observed in their simulations collective motion of weakly bound clusters. ${ }^{390}$ They concluded from their simulations that for some systems concerted cluster movement is responsible for $70 \%$ of the single-file mobility. ${ }^{395}$ Demontis et al. ${ }^{389}$ however, did not observe concerted ethane cluster diffusion in AFI.

\subsubsection{Understanding Chain Length Effects}

We can obtain some insights into the hydrocarbon length dependence of the diffusion coefficient in relation to the zeolite structure, if we consider the free energy barriers. To a first order approximation, the diffusion coefficient is determined by the difference between the free energies of the molecule on top of the barrier and on the bottom of the well. Only if transition state theory would hold would this free energy barrier be sufficient to compute the hopping rate. However, as shown in section 3.2, one also needs to take into account the recrossing rate. Dubbeldam et al. ${ }^{192}$ have shown that for the systems studied in ref 192, the recrossing is to a first approximation a monotonically decreasing function of chain length. This allows us to focus on the free energy difference.

Let us consider the same schematic classification as introduced by Beerdsen et al. ${ }^{351}$ Beerdsen et al. use as a basic building block for a zeolite structure an ellipsoid. These ellipsoids can be connected in three different ways (see Figure 1) to form the most common zeolite topologies.
We obtain the basic unit for a one-dimensional tubelike zeolite by connecting two ellipsoids at the long ends (see Figure $45 \mathrm{~b}$ ). For this configuration the difference between the top of the free energy profile and the bottom is the typical corrugation of the zeolite wall. As the free energy barriers associated with this corrugation generally are small, the diffusion is dominated by the recrossing coefficients and, hence, will monotonically decrease as a function of chain length. This monotonic decrease might be interrupted if the molecule becomes longer than the ellipsoid; from this chain length on, there will always be part of the molecule on top of the barrier and hence the free energy barrier associated with the corrugation will completely disappear. If this effect is sufficiently strong such that it will offset the decrease of the recrossing coefficient, one might see an increase of the diffusion coefficient at exactly this chain length.

A cagelike zeolite is constructed by connecting the ellipsoids at the short ends (see Figure 45a). For this situation we can expect the complex chain length dependence that gives a so-called window effect, which is discussed in section 6.6.2. From a free energy point of view, molecules preferentially adsorb at those sites that have a low free energy, i.e., the bottom of the well, and hence the chain length dependence of the low free energy sites is similar to that of the Henry coefficient. For cagelike zeolites the Henry coefficient increases until the molecule perfectly fits in the cage, and for longer chains the Henry coefficient decreases. The top of the barrier is the window region, and this region affects only a limited number of beads of the chain; the rest of the chain can be in a low energy conformation. Hence, beyond a certain chain length, the free energy of a molecule on top of the barrier follows the same trend as the bottom of the well. So, both the top and the bottom of the free energy profile have a complex nonmonotonic behavior that depends on the details of the size of the cage and the shape of the window region, which may lead to a decreasing, increasing, or nearly constant diffusion coefficient if the chain length is increased.

Finally, one can obtain two- or three-dimensional channels by connecting the long end of one ellipsoid to the short end of the other one (see Figure 45c). In this case the barrier is purely entropic; for the molecule there is no preference in which ellipsoid to adsorb. However, the free energy profile is very different. To see this, consider, for example, diffusion in the horizontal direction, for which the vertically oriented ellipsoids are entropic traps. The average free energy of a molecule is exactly the same in the two elliposoids, but in a free energy profile one uses the free energy per unit length. In the direction of the diffusion, the free energy per unit length is lower for the vertically oriented ellipsoid, as in this orientation the free energy is divided by the short diameter of the ellipsoid, while for the horizontally oriented ellipsoid the free energy is divided by the long diameter. To see how these free energies depend on the chain length, we can use the fact that in these two- or three-dimensional channels the Henry coefficients increase monotonically with chain length; hence, one can expect the free energies in the two ellipsoids to decrease accordingly. Expressed per unit length along the diffusion path, the free energy of the bottom of the well decreases more than the top of the barrier. Therefore, the barrier for diffusion is expected to increase as a function of chain length. Interesting effects can be expected if the chain length approaches the repeating lengths of the channels. 
The above arguments are based on a very simple model of a zeolite. A real zeolite can have much more complex topologies. Nevertheless, this simple model gives us some basic insights into why such a complex chain length dependence is to be expected for the diffusion coefficient and how this diffusion behavior can be related to the Henry coefficients.

\subsection{Mixture Diffusion}

Simulation studies on mixture diffusion in zeolites have been performed on both lattice models and continuum models. The lattice models use a kinetic Monte Carlo scheme (see section 2.6) in which the components are given different hopping rates. Examples of systems that have been studied using this approach include mixtures of $\mathrm{CH}_{4}$ and $\mathrm{CF}_{4}{ }^{396}$ mixtures of 2-methylhexane and a second component, ${ }^{397}$ or binary mixtures of model fluids ${ }^{398}$ in MFI. Such a kinetic Monte Carlo approach is computationally very efficient and has therefore as an important advantage that very detailed studies can be made on well defined model systems. A much more CPU-intensive route is to use molecular dynamics simulations, which have been applied to study mixtures of $\mathrm{CH}_{4}$ and $\mathrm{CF}_{4},{ }^{399} \mathrm{CH}_{4}$ and xenon, ${ }^{400,401}$ and $\mathrm{CH}_{4}$ and butane 226,227 in the zeolite MFI, mixtures of $\mathrm{CF}_{4}$ and $n$-alkanes ${ }^{259,402}$ and mixtures of $\mathrm{Ar}$ and $\mathrm{Kr}$ in FAU, and mixtures of $\mathrm{CF}_{4}$ and neon, ${ }^{390}$ mixtures of methane and ethane, ${ }^{403}$ and mixtures of various hydrocarbons in $\mathrm{AFI}\left(\mathrm{AlPO}_{4}-5\right){ }^{228,318,345}$

Snurr and Kärger ${ }^{399}$ compared molecular dynamics simulations with NMR-pulse field gradient experiments for $\mathrm{CH}_{4}-\mathrm{CF}_{4}$ mixtures in MFI. Whereas the experimental diffusion coefficients for methane agreed quantitatively, the diffusion coefficient of $\mathrm{CF}_{4}$ was underestimated. Since a similar underestimation was observed for the pure component, if one corrects for this difference in pure component diffusion, the simulation results for the mixtures are in excellent agreement with the NMR experiments. For a given total loading of the diffusion coefficient, if the concentration of the less mobile species $\left(\mathrm{CF}_{4}\right)$ is increased, both diffusion coefficients decrease. Qualitatively similar results were also found by Gergidis et at., ${ }^{226,227}$ using both simulations and experiments, for butane-methane mixtures in MFI. Interestingly, although this is intuitively a very reasonable result, it is not universal. For example, Jost et al. performed a similar study for methane xenon ${ }^{400,401}$ mixtures in silicalite. For this system good quantitative agreement of the simulations with the experiments was obtained. Both the experiments and simulations show that the presence of xenon decreases the diffusion coefficient of methane up to a factor of four at high loadings, while in this case the diffusion coefficient of the less mobile component $(\mathrm{Xe})$ was only slightly influenced by increasing the concentration of methane. Such a slight influence on the less mobile component was also observed experimentally for methane/ethane in $\mathrm{MFI}^{404}$ and FAU. ${ }^{405}$ These different examples illustrate that for multicomponents it is difficult to even qualitatively predict the effect of the other components. PFG NMR experiments for diffusion of $\mathrm{nC}_{4}-\mathrm{iC}_{4}$ mixtures in both $\mathrm{FAU}$ and MFI are found to be in good agreement with MD simulations. ${ }^{339}$

The question of how accurately mixture diffusion can be estimated on the basis of the pure component information has been addressed by Krishna and van Baten. ${ }^{261}$ Their starting point is the Onsager transport coefficients $[L]$ (cf. eqs 44 and 45). Krishna and van Baten investigated in detail diffusion of several gas mixtures in several types of porous materials (zeolites, MOFs, nanotubes) and systematically investigated different methods of relating the mixture Onsager coefficients to those of the pure components. Krishna and van Baten show that for a mixture in which there is a homogeneous distribution of the components in the pores, a reasonable estimate can be made for the mixture behavior using phenomenological theories. The phenomenological approach of Krishna and van Baten ${ }^{261}$ has been used to successfully model mixture permeation across zeolite membranes. ${ }^{322}$ However, in the case that there is molecular segregation, in which the two components have very different adsorption sites, the conventional method of predicting the diffusion properties of the mixture fails.

\subsubsection{In-Silico Design of a Separation Process}

An interesting question is whether one can use molecular simulation to screen for zeolite for a particular separation process. This approach has been used for the separation of linear and branched hydrocarbons, ${ }^{406,407}$ of mixtures of linear alkanes, ${ }^{193}$ and of methane and carbon dioxide. ${ }^{408}$ For example, the simulations predict that, for the separations of linear and branched hexane isomers, MFI is a very good candidate. ${ }^{406,407}$ Krishna et al. predict that one can take advantage of the cage effects in zeolites such as CHA, AFX, or ERI to separate linear hydrocarbons that have slightly different lengths. ${ }^{193}$ For the practically important problem of separation of $\mathrm{CO}_{2}$ from natural gas mixtures, the simulation indicates that $\mathrm{CHA}$ and DDR are very promising structures. 408

It is interesting to see that simulations can make these types of predictions for important "real-life" applications, and it is, of course, very important to see whether these predicts will get an experimental follow-up.

\subsection{Toward Real-Life Zeolites}

Most simulations are carried out on ideal zeolite crystals, while in all practical applications one has to work with "realword" materials in which defects are almost unavoidable. These defects can block part of the pore systems or can be crystals that are twinned. From a computational point of view, these defects are difficult to take into account, as our knowledge of the molecular structure of such a defect is very limited.

Newsome and Sholl ${ }^{409}$ used molecular simulations to study the effect of the grain boundaries separating two twinned crystals on the diffusion coefficient. Gupta and Snurr ${ }^{410}$ studied the effect of pore blockage on the diffusion of hydrocarbons.

Often the differences one observes experimentally in diffusion coefficients obtained from microscopic experiments (NMR) and macroscopic uptake experiments are rationalized in terms of a surface barrier that hinders the entering of molecules into the pore structure. Molecular simulations have been used to investigate the process of molecules entering or leaving a zeolite pore. ${ }^{41-413}$ Arya et al. ${ }^{267}$ studied the surface barrier in AFI. Snyder and Vlachos ${ }^{26}$ used kinetic Monte Carlo simulations to study the effect of the surface on the diffusion of benzene in FAU $(\mathrm{NaX})$. Newsome and Sholl ${ }^{415}$ used the dual control grand-canonical Monte Carlo simulations to study the effect of the surface barrier on the diffusion of $\mathrm{CH}_{4}$ and $\mathrm{CF}_{4}$ in MFI. 


\section{Toward an Understanding of Shape Selectivity}

\subsection{Introduction}

Shape selective catalysis continues to have a major effect on refining, petrochemical, and fine chemical processes. ${ }^{8,416-419}$ Weisz and Frilette at the Mobil (now ExxonMobil) oil company coined the term "shape selective catalysis" in the 1950 s, when they discovered that only molecules permeable into an LTA-type zeolite were catalytically converted, to the exclusion of others. ${ }^{420-423}$ The concept was rooted in enzymatic catalysis, ${ }^{421,422}$ which would suggest that explanations for shape selective catalysis would relate to modifications of the transition state for various reactions. ${ }^{424}$ However, explanations for shape selective catalysis gravitated strongly toward mass transport phenomena at Mobil. ${ }^{423,425}$ Upon instigation by Venuto (Mobil), ${ }^{426}$ Csicsery at the Chevron oil company developed the concept of transition state shape selectivity. ${ }^{426-431}$ Interestingly, the seminal study on transition state shape selectivity ${ }^{426}$ does not rigorously exclude mass transport effects. In our view, this did not happen until an elegant study by Haag, Lago, and Weisz from Mobil. ${ }^{432}$ Interestingly, a recent quantum chemical study by Sauer and co-workers et al. ${ }^{44}$ indicates that the conclusions of the seminal study into transition state shape selectivity ${ }^{426}$ were probably incorrect and that mass transfer effects are the dominant cause for the shape selectivity observed. This aside, by the early 1970 s an appealingly simple, "classic" 433 ", concept of shape selectivity had emerged: It was either due to mass transport or due to transition state control of the reactions ${ }^{428,429}$ - even though it was also well established that the phenomenon was considerably more complex. ${ }^{424}$

Again, back in the 1950s, Rabo at Union Carbide (now UOP) started a separate strain of research into shape selectivity by studying the remarkably high activity of FAUtype zeolites ${ }^{434}$ as compared to amorphous aluminosilicates in alkane hydroconversion. In the late 1970s, Rabo recognized that the activity is remarkably high because FAU-type zeolites adsorb and thereby concentrate reactants relative to the gas phase. ${ }^{435}$ Although the (radical) reaction that Rabo ended up choosing to illustrate this "concentration effect" is a source for dispute, ${ }^{442}$ the concept itself was successfully extended to other reactions and zeolites by Derouane, who renamed it the "confinement effect".363,443 For more than two decades, this concept was developed not as an instance of shape selectivity but as an instance of a "confinement effect" 444 or "solvent effect". 44,446 These effects did not cause much resonance within the scientific community until Haag (Mobil) added sorption control to mass transport and transition state control as one of the three potential causes for shape selectivity in the mid 1990s. ${ }^{130}$ Working out how to exactly merge adsorption control into shape selectivity set off a flurry of continuing activities that has not come to an end yet. ${ }^{191,247,248,447-453}$ It seems to provide the keys to puzzles that have riddled the classical shape selectivity model right from its inception.

In a 1971 review, Venuto (Mobil) points out that in addition to shape selectivity there are "reactions on external surfaces and special effects". ${ }^{424}$ Whereas progress has been made in establishing and understanding external surface reactions ${ }^{433,454-456}$ (variously called "nest effects" 457 or "pore mouth ${ }^{424,456,458-460 ", ~ a n d ~ " k e y-l o c k ~}{ }^{461-464 ", ~ c a t a l y s i s), ~}$ the (other)"special effects" have mushroomed into a myriad of phenomena, each with their individual name. Apparently, it became fashionable to simply classify the individual catalytic phenomena instead of trying to arrive at a systematic understanding at a molecular level. An early example of these special effects is the "window" or "cage effect", coined by Chen et al. (Mobil). ${ }^{359,424}$ This effect was later merged into the classical shape selectivity model-initially as a form of mass transport shape selectivity ${ }^{359,465}$ and later as a form of sorption shape selectivity. ${ }^{191}$ Santilli and Zones (Chevron) coined the term "secondary shape selectivity" to describe the selective hydroconversion of $n-\mathrm{C}_{6}$ instead of $n-\mathrm{C}_{16}$ by AFX-type zeolites. ${ }^{466}$ We showed this to be part and parcel of the "window or cage effect" and therefore of sorption shape selectivity. ${ }^{191}$ Derouane postulated that "molecular traffic control" might occur when small molecules can diffuse through small channels and when large molecules can diffuse through large channels of one and the same molecular sieve. ${ }^{467,468}$ Corroborating its existence experimentally will be a challenge. ${ }^{329,417}$ Van Nostrand and co-workers (Chevron) coined the term "inverse shape selectivity" to denote the accelerated formation rate of reaction intermediates that have a shape more commensurate with the framework topology than others. ${ }^{469-471}$ Even though quantum chemical simulations indicate that this form of transition state shape selectivity is conceptually feasible, ${ }^{32}$ the original example turned out to be a form of sorption shape selectivity and not of transition state shape selectivity. ${ }^{248}$

In this review, we try to further integrate adsorption controlled shape selectivity into mass transport and transition state controlled shape selectivity. The resulting systematic understanding affords folding a remarkable number of "special effects" back into the (expanded) notion of shape selectivity.

\subsection{Conventional Hydroconversion Mechanisms}

Any zeolite built of silica only is neutral, but replacing $\mathrm{Si}^{4+}$ by $\mathrm{Al}^{3+}$ creates a negative charge on the framework. When protons neutralize framework charges, they constitute acid sites that can catalyze the two types of reaction underlying all oil refining: isomerization and cracking of hydrocarbons. ${ }^{7}$ Depending on the topology of the zeolite used and the selectivity it imparts, isomerization and cracking reactions form desired products by converting simple $n$ alkanes into different branched isomers and cleaving large hydrocarbons into smaller ones, respectively. ${ }^{6}$ Before addressing the effect of shape selectivity on these reactions, it is worthwhile to first discuss what occurs in the absence of shape selectivity.

At this point it is important to mention that we focus here on the simplest of cracking reactions, which involve the metal-catalyzed activation of feed molecules through hydrogen $\left(\mathrm{H}_{2}\right)$ abstraction and the metal-catalyzed deactivation of product molecules through hydrogen addition. When this metal functionality is absent (as in catalytic cracking) or defective, various functionalities can accomplish the hydrogen subtraction and addition, the system becomes much more complex, and it becomes commensurately more difficult to identify shape selectivity unambiguously.

\subsubsection{Basic Mechanism}

In alkane hydroconversion, a metal site dehydrogenates alkanes into an alkene, and an acid site converts the alkene into another isomer or a cracking product, whereupon the metal site hydrogenates the converted alkene back into an alkane. ${ }^{472-474}$ The hydroisomerization reactions convert 


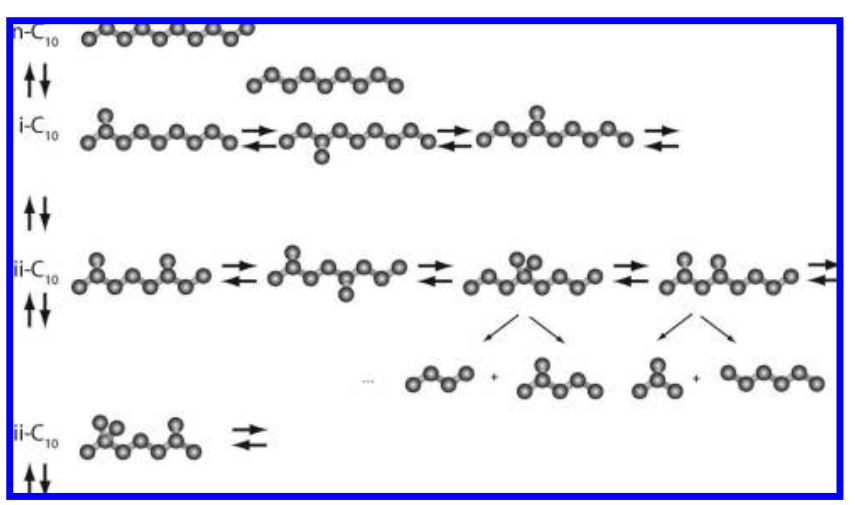

Figure 51. Scheme illustrating some of the chemical reactions that can take place inside the pores of a zeolite. These include hydroisomerization reactions that convert $n$-decane $\left(n-\mathrm{C}_{10}\right)$ into its monobranched $\left(\mathrm{i}-\mathrm{C}_{10}\right.$ ), dibranched (ii- $\mathrm{C}_{10}$ ), and tribranched (iii$\left.\mathrm{C}_{10}\right) \ldots$ isomers. A hydrocracking precursor is formed when hydroisomerization yields a molecule with two branches attached to the same or to next-neighboring carbon atoms. Precursors with two branches hydrocrack relatively easily into a smaller linear and branched alkane. These isomers or their cracking products either continue to react or leave the zeolite as part of the product distribution.

linear hydrocarbons ( $n$-alkanes) into branched isomers; these can then be converted further through transfer of the branch along the molecular backbone, or as a series of consecutive hydroisomerization steps, each increasing the degree of branching. ${ }^{472,475,476}$ Hydrocracking reactions break a hydrocarbon reactant into two smaller molecules and proceed particularly easily if hydroisomerization has formed a socalled hydrocracking precursor: a molecule with two branches that are attached to the same carbon atom or to next-nearestneighboring carbon atoms. The detailed mechanism of the elementary hydrocarbon hydroconversion steps is well understood. ${ }^{477}$ However, the large number of possible reactions and the fact that many molecules may act as intermediates or end up as products typically result in a complex distribution of product molecules that is not simple to predict.

Figure 51 illustrates hydroisomerization and hydrocracking reactions for a simple starting material (or "feed") of pure decane. Suppose we carry out a hydrocracking reaction in sulfuric acid or with unstructured (amorphous) aluminosilicates. Because the (gas-phase) free energies of formation of the various decane isomers shown are nearly identical, the product distribution after cracking will reflect the probability with which particular cracking precursors form. If all isomers form with equal probability, then simple statistical arguments ${ }^{478}$ predict that the highest number of reaction paths lead to hydrocracking precursors that have methyl groups close to the center of the molecule. As illustrated in Figure 52 , such a molecule will be cut or cracked at the methyl groups. The net result is that the product molecules have a Gaussian size distribution centered on half the length of the feed molecule. Such ideal product distributions simply reflect the statistical probability of forming intermediates and product molecules, and they are obtained with hydroprocessing catalysts that do not show shape selectivity.

In addition to the hydroisomerization reactions that change the degree of branching, there are also those that change the distribution of branching toward thermodynamic equilibrium. ${ }^{479-481}$ None of the hydroisomerization reactions equilibrate completely because they compete with consecutive hydrocracking reactions that decompose the isomers. ${ }^{476,478-481}$ The probability of a molecule undergoing a hydrocracking reaction

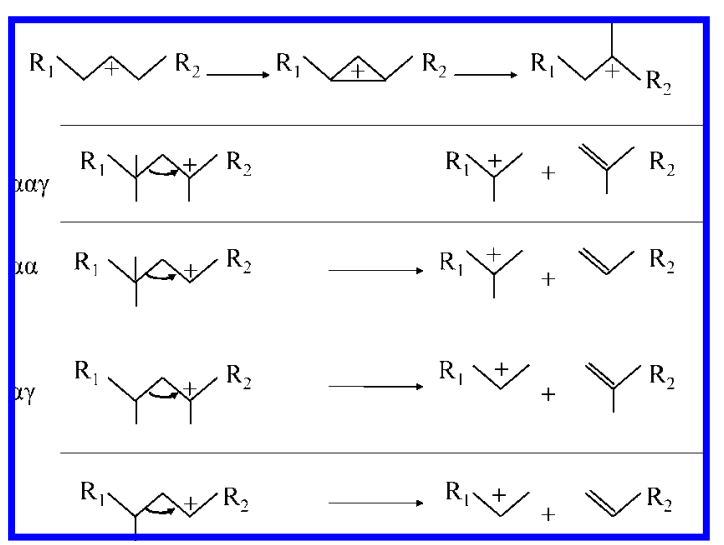

Figure 52. Transition states in hydroisomerization and in the hydrocracking mechanism of the isomers that hydrocrack most easily. ${ }^{476,478-481}$ Hydroisomerization of a linear $n$-alkane into a branched alkane (top, $\mathrm{R}_{1}, \mathrm{R}_{2}$ a $\mathrm{H}\left(\mathrm{CH}_{2}\right)_{x}$-moiety with $x \geq 0$ ) involves a protonated dialkylcycloalkane intermediate. $\alpha \alpha \gamma$-trimethylalkane hydrocracking involves only the most stable, tertiary carbocation transition states and therefore exhibits the highest hydrocracking rate. $\alpha \gamma$ - and $\alpha \alpha$-dimethylalkane hydrocracking involves both a tertiary and a less stable secondary carbocation transition state and therefore exhibits a lower rate. Monomethylalkane hydrocracking involves secondary carbocations only and therefore exhibits a much lower rate than reactions involving at least one tertiary carbocation.

increases with increasing degree of branching, because more extensively branched isomers afford the formation of more stable carbocationic hydrocracking transition state ${ }^{479,478,480,481}$ see Figure 52. For as long as the molecules are adsorbed, we refer to them as a reaction intermediate. A reaction intermediate can either desorb to become a product or continue to react. Those intermediates that have two or more methyl groups sufficiently close so that they can hydrocrack relatively fast are cracking precursors. Figure 1 also illustrates that, even in an ideal hydroconversion experiment in which one utilizes a single component as feed, the product is a complex mixture of products that originate from the hydroisomerizing and hydrocracking of reaction intermediates. Shape selectivity is used to optimize the product distribution for a given application.

To quantify the type of product distribution one has to expect in the absence of shape selectivity, let us consider the hydroconversion reaction of an $n$-alkane in more detail. Figure 51 suggests that the hydroisomerization reactions allow the formation of any branched isomers. At high and intermediate alkene coverage of the acid sites, $\alpha \alpha \gamma$-trimethylalkene hydrocracking dominates the hydrocracking product slate, because this is the most rapid hydrocracking mode (see Figure 52). When this is the case, the product slate consists of a histogram with a single maximum indicative of preferential hydrocracking at the center of the chain, irrespective of the $n$-alkane feed length. ${ }^{472,474,475,484,485}$ This is because the probability of formation of $\alpha \alpha \gamma$ trimethylalkene hydrocracking precursors is dependent on the proximity of the methyl groups to the center of the chain. ${ }^{474-476,485}$ For reasons of symmetry, there are fewer permutations of their precursor transition state closer to the center. For the system in Figure 51 we can therefore expect a product distribution dominated byproduct originating from 3,3,5-trimethylheptane and from 3,5- and 3,3- and 4,4dimethyloctane (see Figure 53).

In addition to the traditional kinetic $n$-alkane hydroconversion network discussed here, an alternative network was 


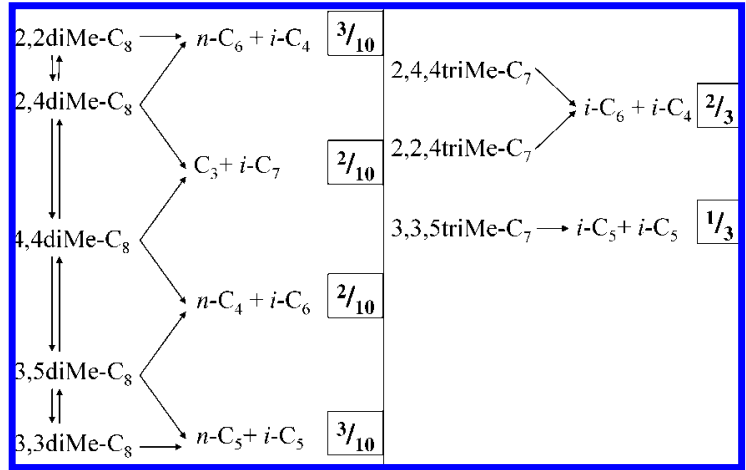

Figure 53. The hydrocracking precursors and products obtained by applying the mechanism shown in Figure 52 to $C_{10}$. In the boxes are the chances for forming hydrocracking products assuming that all $\alpha \gamma$ - and $\alpha \alpha$-dimethyloctanes are available in equal amounts and that there is no preference for hydrocracking. The same was done for $\alpha \alpha \gamma$-trimethylheptane hydrocracking. Only hydrocracking routes involving at least one tertiary carbocation transition state are included because only these routes are fast enough to make an impact. When there are only secondary carbocation transition states involved (as in monomethylalkane hydrocracking), hydrocracking occurs at a significantly lower rate.

proposed. ${ }^{486}$ Both networks agree with the older literature ${ }^{487,488}$ in that the cationic dialkylcyclopropyl transition state plays a key role on hydroisomerization, but they disagree as to the role of these transition states in hydrocracking. The traditional network postulates that the dialkylcyclopropyl transition states only play a role in hydroisomerization and that they have to open and form a full-fledged branched alkene before hydrocracking occurs. ${ }^{475,476}$ The alternative network postulates that the dialkylcyclopropyl transition states do not have to open before hydrocracking sets in and that they themselves can initiate molecular scission. ${ }^{486}$ Both kinetic networks are equally suitable for explaining the hydrocracking product distributions when $\alpha \alpha \gamma$-trimethylalkane dominates, and the hydrocracking product slates resemble Gaussian histograms. ${ }^{476,486}$ Here we use the traditional network as the starting point.

\subsubsection{Traditional Shape Selectivity}

The original notion of shape selectivity is simply the observation that if a molecule cannot permeate through the pores of a zeolite, it will not adsorb as a reactant or desorb as a product. ${ }^{421}$ If a molecule's adsorption is inhibited, it will show up intact in the product slate. If a molecule's desorption is inhibited, it could still form as a reaction intermediate in the adsorbed phase, but only molecules that originate from this reaction intermediate through consecutive reactions will show up in the product distribution.

For example, the FAU-type topology exhibits large cavities in which di- and tribranched hydrocarbons form easily, whereas the TON-type topology exhibits much smaller pores in which only the monobranched isomers form easily. As a consequence, if we use the reaction scheme in Figure 51, the product distribution obtained from TON-type zeolites comprises many monobranched (and some dibranched) isomers and their hydrocracking products, whereas the distribution obtained from FAU-type zeolites comprises many dibranched (and some tribranched) isomers and the hydrocracking products. ${ }^{459}$ Clearly, differently sized and shaped zeolite pores will interact differently with differently sized reactants, reaction intermediates, and products. As a

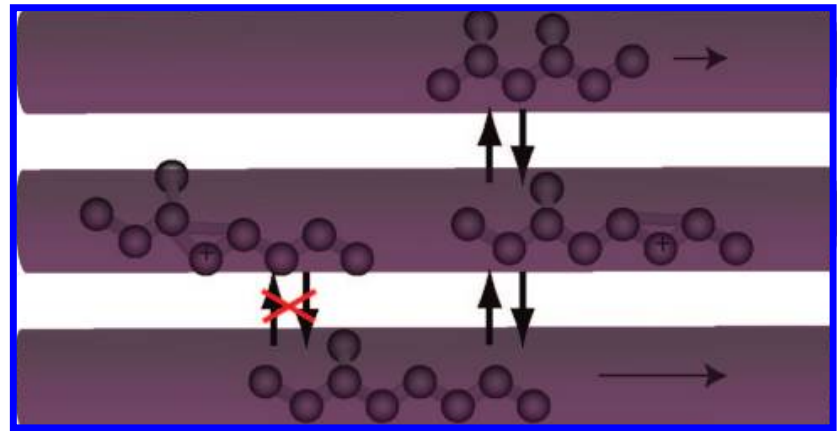

Figure 54. In transition state selectivity the zeolite modifies the ease of formation of a reaction intermediate by modifying the ease of formation of the transition state required for its formation. When a zeolite impedes formation of a reaction intermediate, the Brønsted-Evans-Polanyi principle is likely to apply, so that the zeolite impedes both the formation of the transtition state and that of the resultant (adsorbed) reaction intermediate. ${ }^{32}$ When a zeolite facilitates formation of a reaction intermediate, the BrønstedEvans-Polanyi principle does not necessarily apply, ${ }^{32}$ so that formation of a reaction intermediate can be facilitated without affecting the ease of formation of the transition state. ${ }^{489}$

result, the zeolite topology can leave its"fingerprint" on a particular product distribution.

Although the original concept of shape selectivity is appealingly simple, it can only explain relatively few product distributions. Therefore, forms of shape selectivity other than the originally proposed reactant and product shape selectivity have been put forward. Examples include transition state, reaction intermediate, and exterior surface shape selectivity. In this review we will give a short discussion of these five forms of shape selectivity. In particular, we will use the reaction scheme shown in Figure 51 to illustrate how these various forms of shape selectivity influence the product distribution.

\subsubsection{Transition State Shape Selectivity}

Transition state shape selectivity occurs when a zeolite topology influences the reaction rates of the adsorbed molecules by modifying the relative Gibbs free energies of formation of the corresponding transition states ${ }^{428,429}$ (see Figure 54). It is the only form of shape selectivity that occurs irrespective of the extent of mass transfer limitations between gas and adsorbed phases. ${ }^{433}$ In the reaction scheme of Figure 51, the transition state for hydroisomerization is a dialkylcyclopropyl cation. An example of transition state selectivity is the inability of TON-type zeolites to form (and hydrocrack) $\alpha \alpha$-dimethylalkanes. ${ }^{190,490,491}$ Since the transition states in alkane hydroisomerization occur late in the reaction path, the transition state is sterically similar to the $\alpha \alpha$-dimethylalkane products, ${ }^{190,490,491}$ and similar Van der Waals forces will similarly increase the Gibbs free energy of formation of both the transition states and the products. ${ }^{32}$ This linear relationship between the Gibbs free energy of formation of transition states and that of products is an example of the semiempirical Brønsted-Evans-Polanyi relationship. ${ }^{32,492,493}$ According to this relationship, the prohibitively high Gibbs free energy of formation (and adsorption) of $\alpha \alpha$-dimethylalkane products inside TON-type zeolites ${ }^{190,490,491,494}$ is an indication of a similarly high Gibbs free energy of formation of the transition state for $\alpha \alpha$-dimethylalkane formation, so that transition state shape selectivity will inhibit $\alpha \alpha-$ dimethylalkane product formation inside TON-type zeolite 


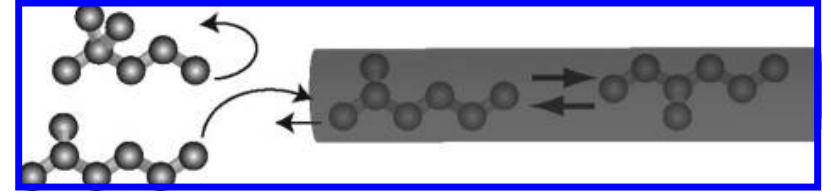

Figure 55. Reactant shape selectivity: the zeolite leaves the dibranched alkane intact because this isomer is too big to fully permeate into the zeolite pores. Instead, the zeolite selectively transforms the monobranched isomers that can fully adsorb.

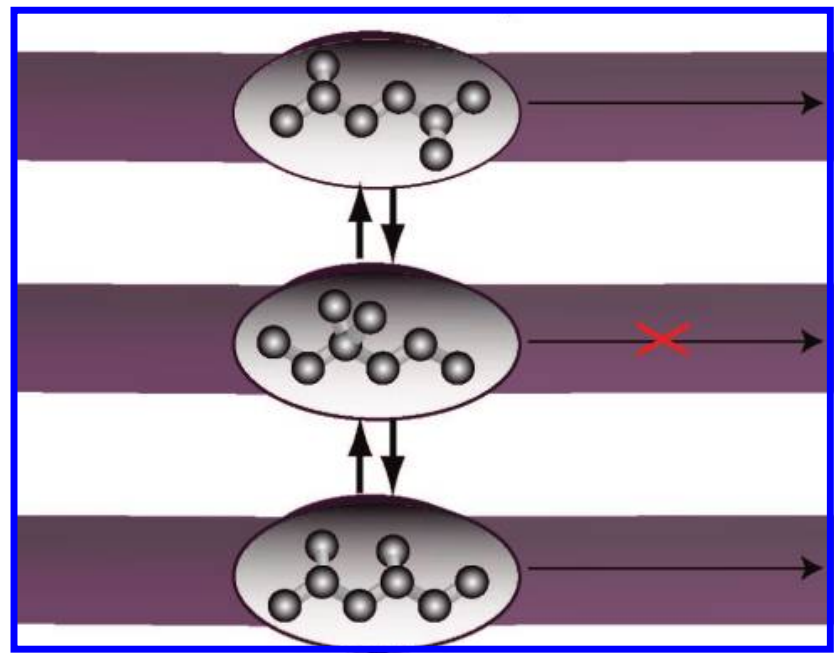

Figure 56. Product shape selectivity: the zeolite does not contribute any dibranched isomers to the product slate because these isomers react much more rapidly than they desorb. Therefore, dibranched isomers cannot leave the zeolite intact. Instead the zeolite selectively yields products that can desorb rapidly.

channels irrespective of the absence or presence of mass transfer limitations. ${ }^{381}$

\subsubsection{Reactant Shape Selectivity}

If any reactant in a feed is too big to permeate a zeolite, this reactant can end up in the product slate virtually intact. This would be an extreme form of reactant shape selectivity (see Figure 55$)^{495}$ in which only those reactants undergo catalytic reactions that can fully adsorb so as to form reaction intermediates, convert, and desorb as products. In general, reactant shape selectivity occurs when some reactants exhibit higher mass transfer limitations than others and therefore achieve a lower conversion than the other, less mass transfer limited reactants. Accordingly, the reactants that adsorb with the smallest mass transfer limitation will be the least prevalent in the product slate. An example of reactant shape selectivity is the selective combustion of only linear and not of branched butanes and butenes on Pt-loaded, $\mathrm{Ca}, \mathrm{Na}-$ exchanged LTA-type zeolite. ${ }^{495}$ Whereas these early experiments involved reactants that are categorically excluded from the LTA-type zeolite pores, ${ }^{495}$ later experiments focused at differences in mass transfer rates as a dominant cause for reactant shape selectivity. ${ }^{432}$

\subsubsection{Product Shape Selectivity}

If reaction intermediates are too big to desorb intact from a zeolite, only their consecutive reaction products can end up in the product slate. This would be an extreme form of product shape selectivity (Figure 56). In general, product shape selectivity occurs when some reaction intermediates exhibit higher mass transfer limitations than others so that they remain in the adsorbed phase and continue to react for a longer period of time than other, less mass transfer limited reaction intermediates. Accordingly, the products that desorb with the smallest mass transfer limitation will be the most prevalent in the product slate.

A typical example of this form of shape selectivity is the cracking of hexane isomers in Ca-exchanged LTA-type zeolites. ${ }^{421}$ It yields only linear and no branched cracking products, because only the former can desorb from the zeolite pores.

\subsubsection{Exterior Surface Shape Selectivity}

In some instances, the exterior surfaces of zeolites process reactants that are too big to adsorb completely ${ }^{191}$ or diffuse too slowly ${ }^{45-456}$ to fully permeate the adsorbate. Whether the exterior zeolite surface has a sufficiently regular structure to yield product distributions different from amorphous aluminosilicates continues to be a subject for debate. Reflecting the lack of agreement on the relevance of the exterior surface to shape selective catalysis, it has been given a plethora of names, such as pore mouth catalysis, ${ }^{456}$ keylock mechanism, ${ }^{417,433}$ and exterior surface shape selectivity. ${ }^{433}$

The relative importance of the zeolite exterior surface is difficult to determine experimentally, as one has to selectively deactivate the exterior surface. One has deactivated the exterior of an MWW-type (see ref 73) zeolite surface to study its impact on the alkylation of benzene with short chain alkenes on MWW-type zeolites. In such a controlled experiment, one can unambiguously establish that these reactions indeed occur predominantly at the exterior surface. ${ }^{454,496}$ However, the postulated mechanism is of the Eley-Rideal type: The acid sites at the exterior surface activate the ethylene or propylene. The activated alkenes leave the acid sites through alkylation of a benzene molecule. ${ }^{497,498}$ This is similar to the mechanism on a nonporous Friedel-Crafts catalyst and strongly suggests that, for this alkylation reaction, shape selectivity does not play a role in this exterior surface catalyzed reaction. Similar control experiments for alkane hydroconversion also suggest a minimal contribution of the exterior surface of $\approx 0.5 \mathrm{~nm}$ TON-type zeolites to the $n$-alkane hydroconversion selectivity. ${ }^{490}$ Apparently, these were considered inconclusive, for papers explaining product distributions by invoking exclusively exterior surface effects continue to be issued. ${ }^{136,458,482,499}$ Maybe the exterior surface effects postulated for $\approx 0.4 \mathrm{~nm}$ ERI-, CHA-, and AFX-type zeolites ${ }^{191,136}$ will stand up better to similar experimental scrutiny.

\subsubsection{Other Forms of Shape Selectivity}

In the previous section we have listed some of the published mechanisms for shape selectivity. In the literature one can find many other types of shape selectivity, such as the "concentration" or "solvent" effect, $435,436,438-441$ the "confinement" or "solvent" effect, ${ }^{444,445,500,501}$ molecular traffic control, ${ }^{467,502}$ secondary shape selectivity, ${ }^{466}$ inverse shape selectivity, ${ }^{469}$ and the "cage" or "window" effect. ${ }^{357,359}$

\subsection{New Mechanism: The Free Energy Landscape}

The zeolite-catalyzed conversion of $n$-decane involves many competing reactions (illustrated in Figure 51), and a 
first step is to identify the preferred reaction path(s). The textbook answer to this question is that one has to look at the free energies of formation. The path that gives the lowest free energy will be the dominant reaction path. A quantification of the system's free-energy landscape is therefore essential, and in particular how this landscape changes with zeolite topology. ${ }^{503}$ The challenge here is that although the gas-phase free energies of formation for most molecules in the reaction scheme of Figure 51 are known and similar, the free energies of formation of the molecules when present in the adsorbed phase in a zeolite are rarely known. Exceptions are molecules that cannot react because they fit nowhere inside a particular zeolite pore; they exhibit a prohibitively large positive free energy of formation. In the case of zeolites with very wide pores, adsorbed molecules will be in physical equilibrium with the gas phase and will probably be unaffected by condensed-phase thermodynamic constraints. But when the fit becomes snugger, molecules formed inside the zeolite may no longer be able to desorb as products and products that have left the zeolite may no longer be able to readsorb; that is, molecules are locked in or locked out. For example, branched alkanes (and alkenes) can contribute the majority of the hydrocracking products to the desorbed product phase while the branched alkane portion of the desorbed phase remains minute. ${ }^{359,504}$

Under such conditions, the gas phase and adsorbed phase can no longer equilibrate and the free-energy landscape imposed by the zeolite topology on the reacting system will leave its signature on the product distribution. Such a lack of equilibration between gas phase and adsorbed phase is endemic to larger molecules in industrial processes such as we consider here. We note that even though complete equilibrium will never be achieved (as in almost all processes), almost all thermodynamic arguments intrinsically assume equilibration. In the context of the present discussion, we argue that, despite the lack of full equilibration, a quantification of the adsorbed-phase free-energy landscape nevertheless serves as a useful starting point that can help us to arrive at a quantitative description of zeolite catalysis. Quantification of the free-energy landscape associated with a particular zeolite topology and particular hydrocarbons has long been impossible but can now be achieved using the sophisticated simulation methods we discussed in section 3 .

\subsubsection{Free Energy Landscape}

In the most general case we have to consider a feed mixture of many reactants. The first step is that these molecules have to adsorb. For a given partial pressure of the various components, the concentration of the molecules depends on the free energies of adsorption. In the absence of diffusion limitations, a relatively low free energy of adsorption of a particular component implies a relatively large contribution of these reactants to the product distribution.

Most experimental adsorption isotherms are determined at room temperature, whereas most of the catalytic activity takes place at much higher temperatures. Therefore, very little experimental data is available at the desired conditions and in most practical applications the concentration of reactant molecules in the pores of the zeolites is unknown. In section 5 we have shown that one can compute adsorption isotherms at any desired temperature. These simulated adsorption isotherms reproduce remarkably well the experimental isotherms at the nonreactive conditions where experiments are feasible. In the case of mixtures, section 5.4 shows that

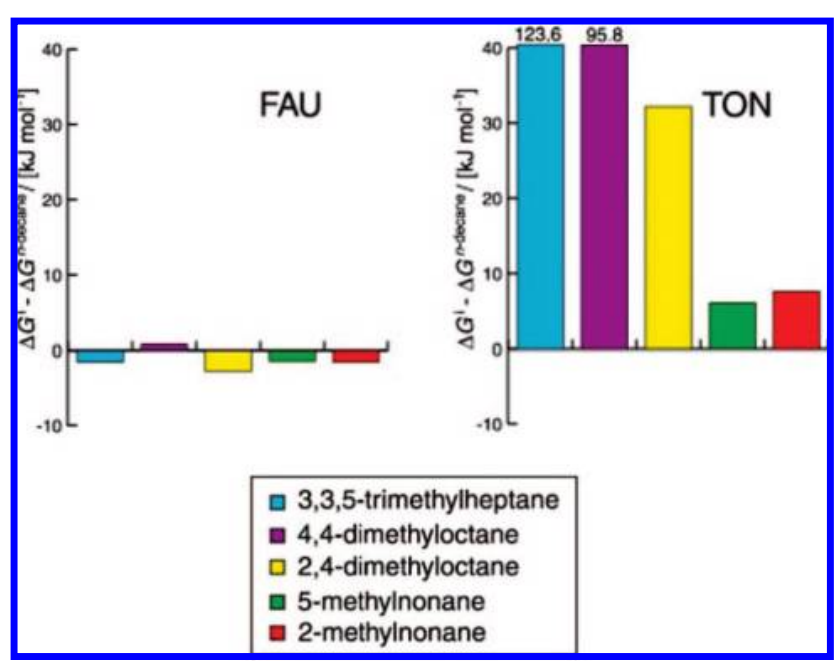

Figure 57. Contributions of the zeolite to the free energy of formation of typical reaction intermediates in the hydroconversion of $n$-decane. The figure gives the free energy difference between the reaction isomer and $n$-decane at $\mathrm{K}$ in the zeolite FAU (left) and TON (right). Figure reproduced with permission from ref 381. Copyright 2001 Wiley-VCH Verlag GmbH \& Co. KGaA.

competitive adsorption can result in very different concentrations of the components inside the pores of a zeolite than one would expect on the basis of the pure component isotherms.

From a simulation point of view, extrapolating the result to the conditions of interest is simply achieved by increasing the temperature. Of course, we are then making predictions on the adsorption behavior that cannot be tested against experimental data. From a computational point of view, the relevant conditions are by no means so extreme that these conditions are completely outside the range of applicability of the intermolecular potentials. The simulated isotherms are fully consistent with the catalytic data at the reactive conditions that preclude experimental determination of isotherms. Despite these uncertainties, simulations are currently the only route to systematically quantify the free energy of adsorption at reaction conditions.

In the following we will simplify the discussion by considering single components. Given the fact that from a computational point of view mixtures do not give fundamental difficulties, we expect that this approach can be extended to mixtures, but this has still to be demonstrated.

\subsubsection{Conventional Shape Selectivity}

The traditional definition of shape selectivity is related to the observation that reactants that are too big to fit inside the zeolite pores do not go on to form products, that transition states that are too big to fit inside the pores of zeolites do not form, and that reaction intermediates that are too big to desorb intact continue to undergo consecutive reactions. ${ }^{433}$

Figure 57 gives the results of the contribution of the zeolite to the free energy of formation of some of the key reaction intermediates. These results give the free energy difference with respect to $n$-decane in various zeolites. Compared to the corresponding gas-phase values, which are nearly identical for all isomers, FAU (a zeolite with large cages) has little effect on the relative free energy of formation of all five molecules investigated. For this system gas phase thermodynamic data are therefore a good approximation. The free energies of formation of alkanes with the same degree of branching are similar, and the resulting ideal Gaussian 
product distribution is simply determined by the number of pathways that generate a particular reaction intermediate; the product slates obtained on amorphous aluminosilicates ${ }^{472,474}$ - and crystalline aluminosilicates with aFAU-type topology ${ }^{475,476}$-are therefore virtually identical.

By contrast, the zeolite TON (with narrow channels) makes a prohibitively high and positive contribution to the free energy of formation of decane isomers with proximate branches. These computations quantitatively confirm the traditional concept of shape selectivity, which predicts that isomers with proximate branches will not form because they are too large for the TON pores. Clearly, one can argue that for this case the differences are so large one does not need to do such simulations; simple geometric arguments would lead to the same qualitative conclusion. However, in case the differences become more subtle, it is important to quantify the differences, as we will see next.

In the previous section we have focused on using the free energy of the reaction intermediates, to evaluate which reaction path dominates. The underlying assumption is that the underlying system is governed by thermodynamic equilibrium. In practice, thermodynamic equilibrium will often not be reached and other factors contribute to the product distribution. Therefore, this free energy model will provide an idealized reference which can be a useful starting point to further investigate the details of a product distribution. As an illustration of this approach, let us consider the hydrocracking of $n$ - $\mathrm{C}_{10}-\mathrm{C}_{17}$ utilizing EMT-, FAU-, MFI-, or BEA-type zeolites. ${ }^{460,505-510}$ The experimental product distributions show a shift from a Gaussian to a multimodal distribution (see Figure 58). This shift results in a decrease in the branched isomer yield and an increase in the combined $\mathrm{C}_{3}$ and $\mathrm{C}_{n-3}$ yield.

This distribution was tentatively explained in terms of shape selectivity: the preferential formation of isomers that somehow were more commensurate with the EMT-, FAU-, or BEA-type topology. ${ }^{460,505-510}$ This hypothesis implies that these topologies should lower the free energy of formation of these isomers significantly. This hypothesis was evaluated by Maesen et al. ${ }^{511}$ Surprisingly, the simulations revealed no significant contribution of these zeolites to the free energy of formation. In addition, none of the isomers exhibited a particularly high Gibbs free energy barrier for diffusion in these materials. This suggested that an explanation other than shape selectivity was called for.

An important factor in reaching thermodynamic equilibrium is the coverage of the acid sites with alkenes. This coverage determines how far an $n$-alkane progresses though the series of consecutive hydroisomerization reactions. ${ }^{472,474,478-481,514,515}$ When more alkenes compete for adsorption at an acid site, the average alkene residence time at an acid site decreases; alkenes will undergo fewer cycles per molecule and will have a higher chance of desorbing intact instead of being cracked before hydrogenating back into an alkane. This adsorption-assisted desorption at high alkene coverage of the acid sites results in a buildup of more mono- and dibranched alkanes that in turn can build up more tribranched alkanes before hydrocracking. Therefore, alkane isomers will progress further toward thermodynamic equilibrium at high alkene coverage than at low alkene coverage. To understand the effect of the alkene coverage on the product distribution, consider the mechanisms shown in Figures 51 and 52. At high alkene coverage, $\alpha \alpha \gamma$-trimethylalkane isomers will dominate the hydrocracking pathways.

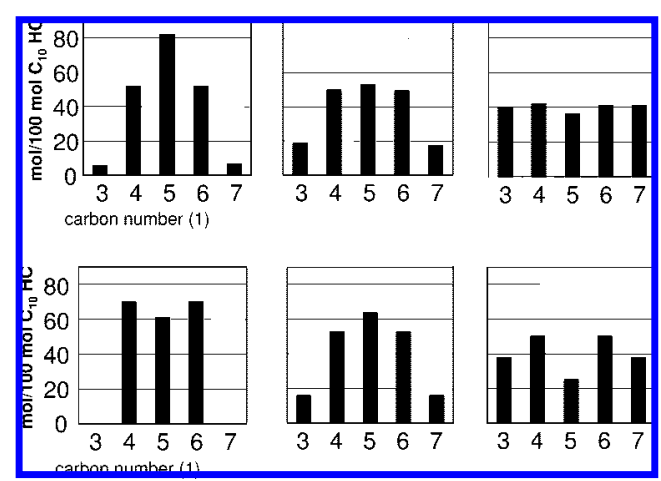

Figure 58. The top figures illustrate the effect on the hydrocracking selectivity of a shift in the dominant hydrocracking pathway. The different pathways are from $\alpha \alpha \gamma$-trimethylalkane (left), to $\alpha \alpha$ - and $\alpha \gamma$-dimethylalkane (middle), and ultimately to monomethylalkane hydrocracking (right). The figures refer to $\mathrm{MOR}^{484}$ (left), to an intergrowth of EMT and FAU ${ }^{507}$ (middle), and to MFI-type zeolites (right), ${ }^{512,513}$ respectively. The bottom figures show the theoretically estimated cracked product distribution of gas phase equilibrium mixtures of (left to right) trimethylheptane, dimethyloctane, and monomethylnonane, respectively. Whereas dimethyloctane and monomethylnonane approach equilibrium before they hydrocrack, trimethylheptane hydrocracks as soon as it is formed, so that for trimethylheptane the likelihood of formation of the isomer determines the distribution.

At lower alkene coverage, $\alpha \alpha$ - and $\alpha \gamma$-dimethylalkane (middle) and, at even lower alkene coverage, monomethylalkane hydrocracking will become more dominant. When $\alpha \alpha \gamma$-trimethylalkene hydrocracking dominates, we expect product slates with a single maximum at the middle of the distribution irrespective of the chain length. When a lower alkene coverage (and higher alkene residence time at the acid sites) shifts the dominant kinetic pathway to the hydrocracking of less branched $\mathrm{C}_{10}$ alkanes (Figures 51, 52), it decreases i- $\mathrm{C}_{5}$ formation and enhances $\mathrm{C}_{6}+\mathrm{C}_{4}$ formation. For $n$-alkanes longer than $n$ - $\mathrm{C}_{10}$, this shift in the dominant kinetic pathway results in a shift from a Gaussian to a flat distribution or even a bimodal product distribution. The remarkable similarity between experimental hydrocracking yield patterns and calculated ones for $n$ - $\mathrm{C}_{10}$ to $n$ - $\mathrm{C}_{17}$ undergoing either predominantly trimethylalkane hydrocracking or predominantly dimethylalkane hydrocracking strongly suggests that such a shift in dominant kinetic pathway explains these yield patterns and not the specifics of the highly disparate EMT-, FAU-, MOR-, and BEA-type topologies. ${ }^{511}$ Figure 58 extends this concept so as to include a shift in dominant kinetic pathway toward monomethylalkane hydrocracking.

At this point it is important to mention that this example illustrates that molecular simulations can be very useful in testing hypotheses underlying proposed reaction mechanisms, but it also nicely illustrates its limitations. In view of the complexity of the kinetic networks that are involved in zeolite catalysis and many other practical factors that contribute to the experimental product distribution, it will be clear that molecular simulations elucidate only part of the complex set of factors involved in shape selectivity.

\subsubsection{Transition State Selectivity}

Transition shape selectivity plays a role when the zeolite topology alters the free energy of formation of a transition state. For many reactions one can assume that the BrønstedEvans-Polanyi (BEP) relation holds. This relation states that the activation energy and reaction energy are related linearly because the transition state and resulting adsorbed reaction 


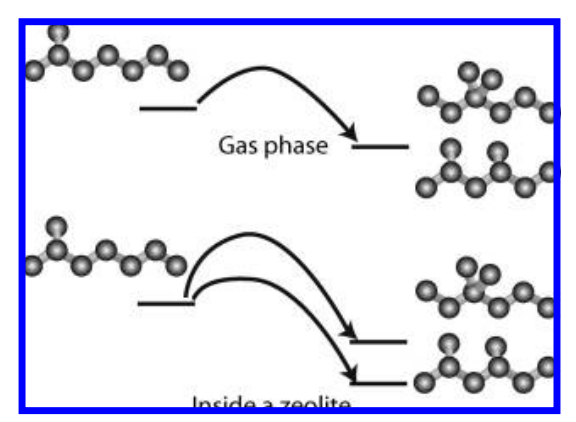

Figure 59. Brønsted-Evans-Polanyi relationship: in the gas phase the free energies of formation of the two products are virtually the same, and so are the free energies of formation of the transition state. In the gas phase the zeolite increases the free energy of formation of one product relative to that of the other product. According to the semiempirical Brønsted-Evans-Polanyi relationship, this increases concomitantly the free energy of formation of the transition state for this product-the alkane isomer with geminal methyl groups in our example.

intermediate exhibit similar steric constraints. ${ }^{32,381,492,493,516-518}$ Hence, if the zeolite increases the relative free energy of one of the reaction intermediates, the corresponding transition state increases similarly. The consequences of this relation are that the differences in the free energies of the transition states of competing reactions can be estimated from the differences in the free energies of formation of the corresponding reaction intermediates (see Figure 59). If we assume that the semiempirical Brønsted-Evans-Polanyi relationship holds for the entire reaction scheme, we can deduce which transition states have an increased free energy of formation in the adsorbed state from the computed free energies of the reaction intermediates.

Frequently, the Brønsted-Evans-Polanyi relationship holds. However, a suggestion is that the Brønsted-Evans-Polanyi relationship might not hold for instances where adsorbentadsorbate Van der Waals interactions decrease the reaction energy in the adsorbed phase. ${ }^{32}$ This would suggest that evaluating the acceleration of reaction rates due to the zeolite topology-induced facilitated formation of a transition state requires a full-fledged quantum chemical evaluation.

Another instance when the Brønsted-Evans-Polanyi relationship might not hold is when the shape of the transition state is very different from the shape of the adsorbed reaction intermediate. In that instance, one needs to investigate the transition state in detail. In acid zeolite chemistry, the transition state is usually associated with some type of carbocation (see, for example, Figure 52). And the key issue is the change of free energy of this carbocationic transition state relative to a reaction intermediate as induced by the zeolite framework. For example, van der Waals interactions between a transition state and the zeolite framework can decelerate the reaction by increasing the free energy of formation of transition states that are incommensurate with the particular zeolite topology. ${ }^{427-430,432}$ Alternatively, ionic interactions between a transition state and the zeolite framework can accelerate the reaction by decreasing the free energy of formation of transition states that are commensurate with the particular zeolite topology. ${ }^{32}$

To quantity these effects, one has to perform a detailed quantum chemical calculation in the pores of the zeolite and to determine the transition state in the pores. These are often very time-consuming calculations and are usually limited to a small part of the zeolite. Only a few studies have been published that take the full zeolite structure into account. ${ }^{32,519}$
In addition, as we have discussed in section 4.4.1, the acid sites can be distributed over various crystallographically different positions and one has to investigate each of the different locations of the acid site, as the transition state may depend on the location of the acid site. At present, these quantum chemistry calculations are too time-consuming for a systematic investigation for all zeolites. Methods are being developed to integrate these quantum chemistry calculations using embedded methods with force-field-based methods.

In the following we assume that the BEP relation holds and that the shift of the free energies of the reaction intermediates is a measure for the changes in the activation energies associated with the corresponding transition states.

Transition state shape selectivity is observed in TON. We have seen in Figure 52 that the free energy calculations of reaction intermediates in TON-type zeolites (Figure 52) indicate a significant influence on the product distribution that is not found for FAU-type zeolites. In the relatively small TON-type pores, $\alpha \alpha$-dibranched and $\alpha \alpha \gamma$-tribranched decane isomers have a prohibitively large free energy of formation, so that these isomers will not form inside this zeolite. This leaves monobranched alkanes as predominant reaction intermediate and product. The currently available experimental data suggest that TON-type zeolites impede formation of reaction intermediates with proximate methyl groups irrespective of the presence or absence of mass transfer limitations between adsorbed and desorbed phases. This would imply that this is an example in which a high free energy of formation of the reaction intermediate goes hand in hand with a high free energy of formation of the relevant transition state-as perthe semiempiricalBrønsted-Evans-Polanyi relationship. This would make the absence of $\alpha \alpha$-dibranched alkanes and their hydrocracking products an instance of transition state shape selectivity.

Of course, in the case of FAU- and TON-type zeolites, the free energy differences are so large that one does not have to perform a molecular simulation to understand that the relevant tribranched decane isomers fit in a FAU-type topology but do not fit in a TON-type topology. However, for some molecules the differences are more subtle, so that quantification of these differences by Monte Carlo simulations can contribute to a significant improvement of the contribution of the topology to the catalytic selectivity.

As a second example of transition state shape selectivity, consider the alkane conversion in MFI-type pores. Molecular simulations have shown (see Figure 60) a large and positive contribution of these zeolites to the free energy of formation of $\alpha \alpha \gamma$-trimethylalkanes. ${ }^{381,520}$ By comparison, these materials impede the adsorption and formation of $\alpha \gamma$-dimethylalkanes only to a small extent. ${ }^{381,520}$ They do not impede the formation of $\alpha \alpha$-dimethylalkanes, monomethylalkanes, and $n$-alkanes, for the shape of these isomers is commensurate with that of the MFI-type intersections, so that all have a similar free energy of formation in the adsorbed phase. ${ }^{381,520}$ Accordingly, these free energies indicate that the shape selectivity in MFI-type pores is dominated by consecutive hydroisomerization of $n$-alkanes into monomethylalkanes and $\alpha \alpha$-dimethylalkanes. Since the favored $\alpha \alpha$-dimethylalkanes are hydrocracking precursors which have a low diffusion coefficient, the net result would be that the shape selectivity inside MFI-type pores enhances the hydrocracking rate at the cost of the hydroisomerization rate.

The major difference between the TON- and MFI-type pores is that the former do not intersect. ${ }^{1}$ The absence of 


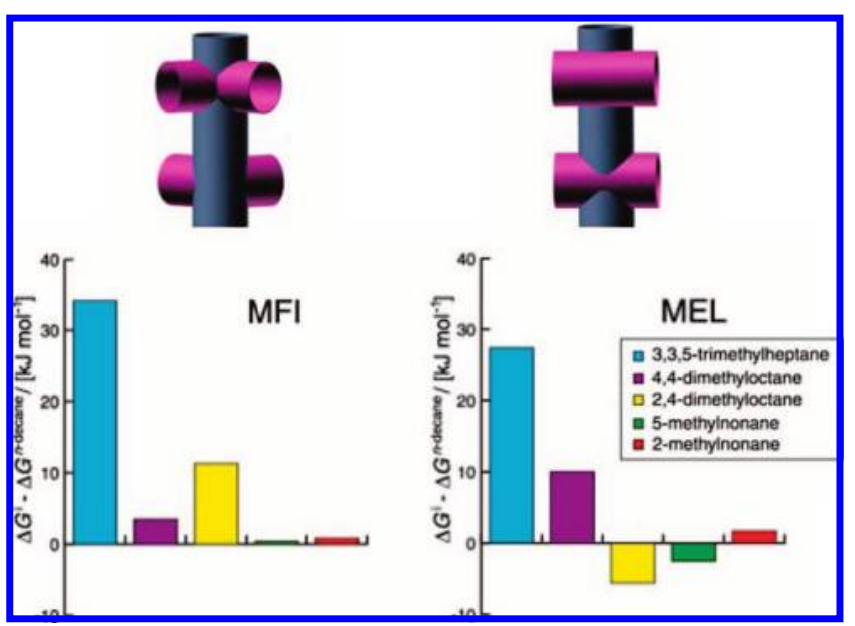

Figure 60. Comparison of MFI- and MEL-types of pores: top, an artist's impression of the structures; bottom, free energy of formation of selected reaction intermediates relative to that of adsorbed $n$-decane. Figure reproduced with permission from ref 381. Copyright 2001 Wiley-VCH Verlag GmbH \& Co. KGaA.

intersection has a major effect on the distribution of reaction intermediates. Comparison of the free energies of the reaction intermediates in TON (Figure 57) and MFI (Figure 60) shows that TON cannot accommodate the formation of either $\alpha \alpha \gamma$ trimethylalkanes or $\alpha \alpha$-dimethylalkanes. ${ }^{190,381,490,491,494} \mathrm{TON}-$ type pores hamper the formation of $\alpha \gamma$-dimethyloctanes and slightly hamper the formation of methylnonane from $n$ decane. ${ }^{381}$ The net result is that TON-type pores impede the formation of the trimethyl- and dimethylalkane isomers that hydrocrack most easily, and therefore decrease the hydrocracking rate in favor of the hydroisomerization rate. ${ }^{190,490,491}$

Let us now compare the product distributions obtained from decane conversion in MFI- and MEL-type pores. Figure 60 shows that the structures of these two zeolites are remarkably similar; the main difference is that MFI has both sinusoidal and straight channels whereas MEL has only straight channels. Despite these similarities, the decane cracking product distribution is very different; the isobutane yield of MEL is twice that of MFI-type zeolites. ${ }^{381,520}$ Figure 60 shows that the computed free energies of formation for $\alpha \alpha \gamma$-trimethylalkanes are highly repulsive, to the extent that their Gibbs free energy of formation in the adsorbed phase effectively prohibits formation of these hydrocracking precursors in both zeolites. In the absence of $\alpha \alpha \gamma$-trimethylalkanes, $\alpha \alpha$ - and $\alpha \gamma$-dimethyloctanes are the most likely hydrocracking precursors ${ }^{520}$ (see also Figures 51 and 52). Interestingly, the free energy calculation indicates $\alpha \alpha$ dimethyloctanes have a relatively low free energy at MFItype intersections, suggesting that these intermediates are commensurate with the shape of the MFI-type intersections, whereas $\alpha \gamma$-dimethyloctanes are commensurate with the shape of one of the MEL-type intersections. Thus, 4,4dimethyloctane fits snugly when it has its octane backbone in the straight MFI-type channel and the two methyl groups in the zigzag channel, and 2,4-dimethyloctane has a perfect fit in the large MEL-type intersection because the distance between the two branches matches the distance between the two intersecting channels (see Figure 61). The commensurate isomers have the lowest free energy of formation. ${ }^{381,520}$ Due to the relatively large zeolite crystals and high acid site densities used, ${ }^{521,522}$ alkanes with the lowest free energy of formation are preferentially formed but cannot diffuse out of the zeolite without being hydrocracked. ${ }^{381}$ Since hydro-

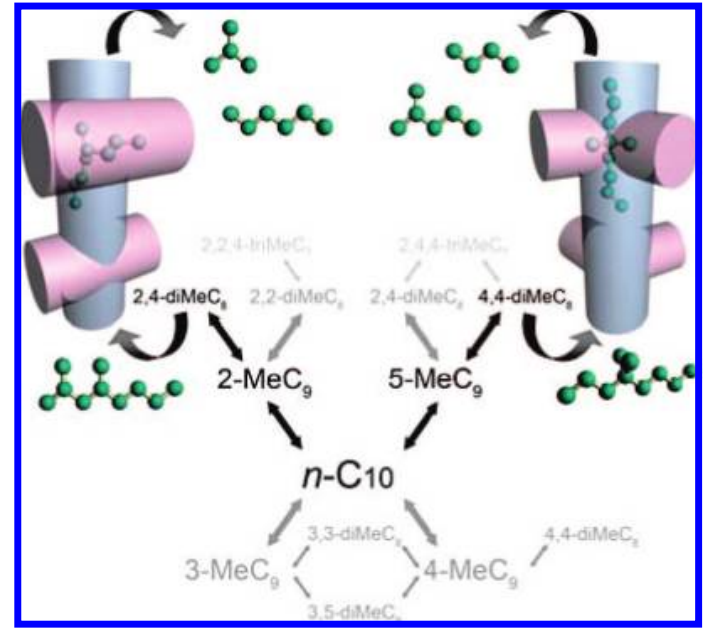

Figure 61. Reaction intermediate shape selectivity: the zeolite is favoring the formation of those reaction intermediates that are more commensurate with the structure of the zeolite at the expense of those that are less commensurate. The more commensurate reaction intermediates have a lower free energy of formation so that they are formed preferentially. However, they have too low a diffusion rate and too high a reactivity to desorb intact, so that their predominance only shows up in the consecutive hydrocracking product slate. (Figure reproduced with permission from ref 503).

cracking of 2,4-dimethyloctane yields isobutane, while hydrocracking of 4,4-dimethyloctane yields $n$-butane, the preferential formation of commensurate isomers suggests an explanation for the twice as high isobutane yield of MELas compared to MFI-type zeolites. ${ }^{381,520}$ Attributing the difference in butane yield to a difference in dimethyloctane hydrocracking precursor is relatively straightforward. Doing the same with the hexane and heptane fractions has been done but is more speculative due to the interference of posthydrocracking hydroisomerization reactions. ${ }^{520}$

This example nicely illustrates the concept of the free energy landscape approach; the zeolites preferentially form reaction intermediates that have a low free energy of formation in the adsorbed state. In the case of MEL- and MFI-type zeolites, the important reaction intermediates are those that are commensurate with the zeolite structure and therefore have an unusually low free energy in the adsorbed phase. They can only form at the intersections, for they have a very large free energy at any other position. This comparative example illustrates how zeolites can control product distributions by favoring the formation of particular reaction intermediates. We also note that both reaction intermediates are typical "ship-in-a-bottle" molecules that can form inside a zeolite but not desorb. Any attempt to experimentally determine the adsorbed-phase free energies of such molecules is a major challenge. Simulations may thus be the only viable means for obtaining the information that is needed to develop a mechanistic explanation for the different catalytic properties of topologically similar zeolites such as MEL and MFI. So far these reactions have only been studied for severely diffusion limited MFI- and MEL-type zeolite crystals. Very small MFI-type crystals have become available ${ }^{523-526}$ that should afford redoing the experiments with minimal mass transfer limitations and establishing to what extent mass transport effects interfere with pure transition state shape selectivity. Interestingly, the explanation for the difference in product slate between MFI- and MEL-type zeolites does not necessarily involve transition state shape selectivity. In principle, the MFI- and MEL-type product slates can also 


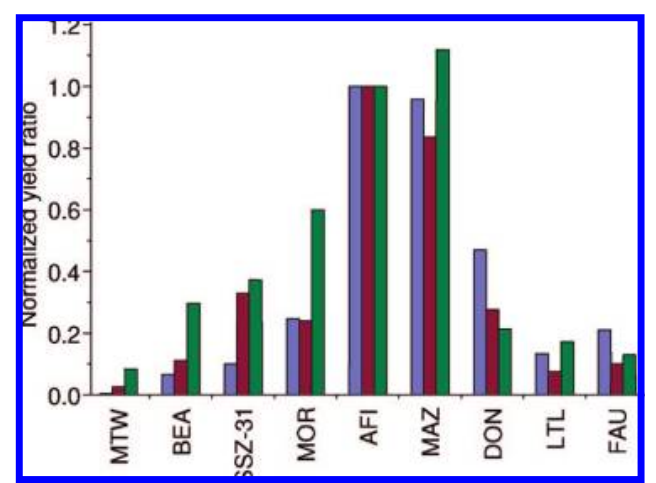

Figure 62. Effect of pore size on the hydroisomerization selectivity (2,3-dimethylbutane-to- $n$-hexane ratio) of the $C_{6}$ hydrocracking products formed during $n$ - $\mathrm{C}_{16}$ hydroconversion (increasing pore size from MTW to FAU). When the pores are small (as with MTWtype zeolites), repulsive adsorbent-adsorbate Van der Waals interactions impede dimethylbutane (DMB) formation; when the pores increase in size, those impeding interactions disappear and interadsorbent interactions favor formation of the better packing DMB; when the pore size increases above $0.74 \mathrm{~nm}$, differences in packing efficiencies disappear because the adsorbents no longer have to line up head-to-tail but can pack in an increasingly more random, liquid-like fashion.

be the result of a similar chemical equilibration toward the thermodynamically favored decane isomer distribution at the MFI- and MEL-type intersections. It is clear that the difference in hydrocracking product distribution is the signature of a difference in decane isomer distribution in the adsorbed phase. What is not clear at this stage is if the difference in isomer distribution is caused by a difference in isomer formation kinetics (different free energies of formation of the transition states) or in isomer formation thermodynamics (different free energies of formation of the adsorbed isomer itself). We have coined this new form of shape selectivity "reaction intermediate shape selectivity". The distinction between "transition state shape selectivity" and "reaction intermediate shape selectivity" can be made more unambiguously in the following example.

Systems in which adsorbate-adsorbate intermolecular interactions play an important role ${ }^{247,248}$ can more unambiguously induce reaction intermediate shape selectivity. Let us consider the effect of pore size on the hydroisomerization selectivity of the $C_{6}$ hydrocracking products formed during $n$-hexadecane, $n$ - $\mathrm{C}_{16}$, hydroconversion. Figure 62 shows that the ratio between dimethylbutane and $n$-hexane yield as a function of pore size is a bell-shaped curve. ${ }^{247,248,469-471}$ These results indicate that there exists an optimal pore diameter for the formation of branched alkanes. This phenomenon is often referred to as inverse shape selectivity; ${ }^{469}$ the zeolite is favoring-instead of excluding-the formation of the most bulky isomers, the dibranched alkanes.

This phenomenon was explained in terms of an optimal fit of the branched alkene reaction intermediate with the zeolite pores. However, simulations have shown that at low pressure such an optimal fit is not reflected in the contribution of the zeolites to the free energy of formation. Interestingly, only at high pressures, where adsorbate-adsorbate interactions are important, such an optimum could be reproduced. ${ }^{247}$ If the pores are too narrow for the bulky dibranched alkane to fit, this will be reflected in a high and positive contribution of the zeolite to the free energy of formation of dimethylbutane relative to $n$-hexane. When the pore size increases toward an optimum size, more dimethylbutane compared to $n$-hexane can fit in the tubular channels, since the dibranched molecule is more compact. Because of these differences in effective size at sufficiently high pressure, the more compact molecule has the lower free energy. ${ }^{240,247,248}$ This size entropy effect is also responsible for differences in adsorption behavior of theses isomers (see section 5.4.2 and Figure 28).

Figure 62 shows that the tubular MAZ- and AFI-type pores share this optimum size for adsorbing and forming dimethylbutane instead of $n$-hexane. ${ }^{247}$ When the pores are larger still, molecules no longer stack linearly so that the adsorbed phase approaches the liquid phase and the entropic size effect vanishes. This difference in packing efficiency leaves its mark on the product slate because slowly diffusing $n-\mathrm{C}_{16}$ locks up the initial $C_{6}$ hydrocracking products sufficiently long to have them approach adsorbed phase chemical equilibrium. Once desorbed, $\mathrm{C}_{6}$ is unlikely to compete with $\mathrm{C}_{16}$ for adsorption in the zeolite, so that no chemical equilibration toward gas phase thermodynamic equilibrium will occur. Interestingly, without $\mathrm{C}_{16}$ one does not observe an optimal selectivity toward dimethylbutane. ${ }^{489}$ In contrast to transition-state selectivity, reaction intermediate shape selectivity requires severe mass transfer limitations between gas and adsorbed phases.

\subsubsection{Reactant Shape Selectivity}

Zeolites can shape selectively process more of one than of another reactant because one of the reactants has a higher diffusion rate, or because one of the reactants has a lower free energy of adsorption. In the former instance of reactant shape selectivity, the reaction needs to be adsorption rate limited before reactant shape selectivity occurs, ${ }^{432}$ in the latter instance, this is not a prerequisite for shape selectivity. In section 5.4 we have seen that the relative concentration of different molecules inside a zeolite can be remarkably different from that outside the zeolite. The free energy of adsorption is a measure for these concentration differences. Experimentally, insights in reactant shape selectivity due to differences in free energy of adsorption have been obtained in studies on the chain length dependence of the reactivity of $n$-alkanes.

An illustrative example is a study of the reactivity of $\mathrm{C}_{8}-\mathrm{C}_{20}$ alkane cracking at very low loading. This reactivity is virtually independent of the chain length, because an increase in the pre-exponential factor compensates for a decrease in the apparent activation energy with chain length. Since it was first described in the $1920 \mathrm{~s},{ }^{527-530}$ the origin of this so-called compensation effect has been the subject of a lot of debate. ${ }^{531}$ Haag et al. ${ }^{130}$ showed that the true activation energy of the cracking reaction is virtually invariant with alkane chain length despite the rapid decrease in the apparent activation energy. The reason for the rapid decrease in apparent activation energy and increase in preexponential factor with chain length is that the enthalpy of adsorption dominates the former and the entropy of adsorption the latter at the catalytic cracking conditions applied. ${ }^{130}$ Provided the pores are large enough, ${ }^{191}$ the adsorption enthalpy decreases drastically with $n$-alkane chain length, ${ }^{130}$ because additional methylene $\left(-\mathrm{CH}_{2}-\right)$ groups bring additional stabilizing adsorbent-adsorbate Van der Waals interactions. These Van der Waals interactions also reduce the number of conformations available to a molecule in the adsorbed as compared to the gas phase, and thereby increase its adsorption entropy. Thus, an increase in adsorption entropy (increase in pre-exponential factor) offsets a decrease in adsorption enthalpy (decrease in apparent activation energy) 


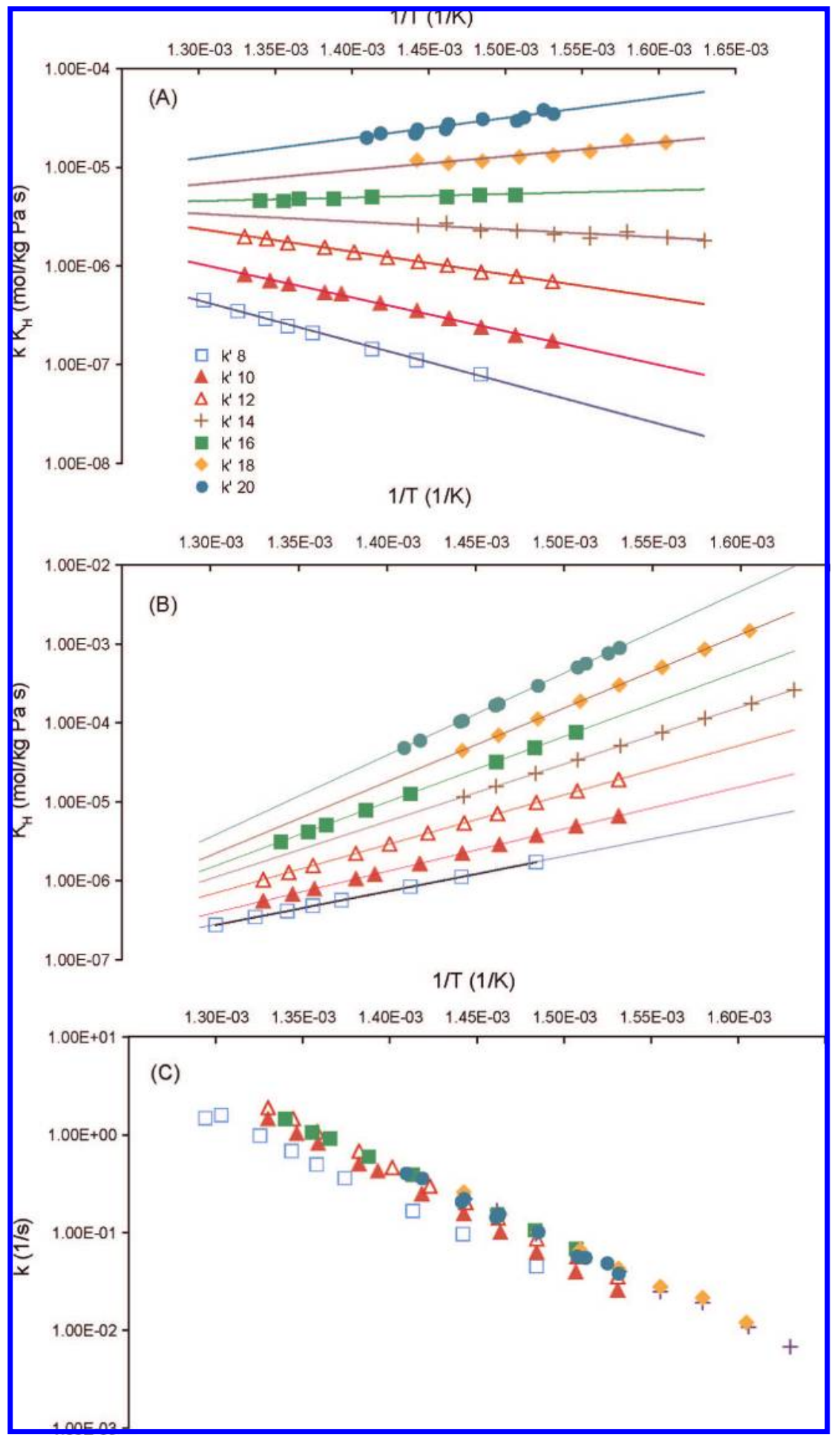

Figure 63. (A) Product of intrinsic reaction rate constant, $k(1 / \mathrm{s})$, and Henry coefficient, $K_{\mathrm{H}}(\mathrm{mol} / \mathrm{kg}$ Pa), observed for cracking $n$-alkanes on MFI-type zeolites at various reciprocal temperatures $1 / T(1 / \mathrm{K})$ in the absence of added hydrogen (adapted from Wei et al. ${ }^{52,452}$ ); (B) Henry coefficient, $K_{\mathrm{H}}$, in MFI-type silica simulated for $n$-alkanes at these reciprocal temperatures; $(\mathrm{C})$ intrinsic reaction rate constants, $k$, as calculated from measured $k K_{\mathrm{H}}$ (part A) and simulated $K_{\mathrm{H}}$ (part B). Figure based on data from ref 504 .

with increasing chain length. If one discounts for these adsorption effects, the (true) pre-exponential factor and activation energy are hardly a function of chain length (see Figure 63). ${ }^{504}$

At most catalytic reaction temperatures, the net effect of an increase in chain length is a decrease in Gibbs free energy of adsorption and therefore an increase in reactivity. ${ }^{447,448,453}$ The degree of compensation between adsorption entropy and enthalpy is a clear function of the pore size, ${ }^{191,194,448}$ so that the discrimination of zeolites between $n$-alkanes of various lengths depends on the pore topology. To the extent that the reactivity of $n$-alkanes as a function of chain length varies with zeolite topology, it is - by definition ${ }^{433}$ - an example of (reactant) shape selectivity.

At low pressure and loading, the Henry coefficient is directly proportional to the free energy of adsorption. The Henry coefficient (see section 5.2.1) is a measure of the pressure required to adsorb a given amount of molecules in the pores of the zeolite. Most zeolites (such as, FAU-, ${ }^{447,482}$ OFF-, ${ }^{533}$ and MFI-type ${ }^{452}$ zeolites $^{448,453}$ ) are similar to 


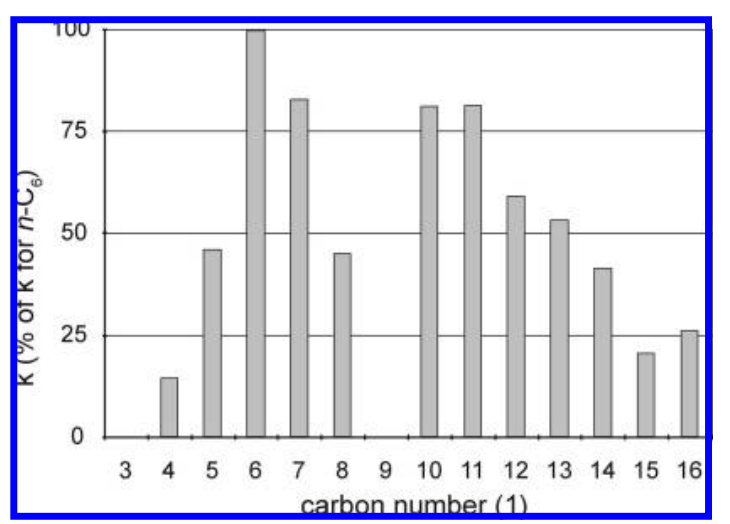

Figure 64. Hydroconversion rates as a function of chain length on ERI-type zeolites as Chen et al. ${ }^{465,534}$ inferred from various mixed feed experiments. Chen et al. assumed that there would be no competitive assumption. This might not be entirely accurate, so that it could be worthwhile to revisit these activities utilizing singlecomponent feeds.

amorphous aluminosilicates in that the Henry coefficient increases monotonically with chain length. A monotonic increase in Henry coefficient with chain length at low pressure is equivalent to a monotonic decrease in free energy and a monotonic increase in adsorbed reactant concentration with chain length. Predominantly as a result of the monotonically increasing alkane concentration with $n$-alkane chain length in the adsorbed phase, the reactivity of $n$-alkanes in hydroconversion increases monotonically with chain length (see Figure 63). ${ }^{447}$ Accordingly, the products originating from the longer $n$-alkanes dominate the product slate. This selectivity for processing longer alkanes is an instance of shape selectivity only for as much as it unambiguously depends on the zeolite pore topology, ${ }^{433}$ i.e. only for as much as it is different for zeolites than for e.g. amorphous aluminosilicates.

Figure 21 shows that for some zeolites the Henry coefficient decreases as a function of chain length. It is interesting to see how this chain length dependence of the Henry coefficient relates to the chain length dependence of the $n$-alkane hydroconversion rate of ERI shown in Figure 64. The ERI-type pore topology exhibits small $0.4 \mathrm{~nm}$ across openings (or "windows") giving access to somewhat larger cages. ${ }^{1}$ From $n$ - $\mathrm{C}_{4}$ to $n$ - $\mathrm{C}_{6}$, ERI-type zeolites exhibit the usual increase in reactivity due to a combination of a lower free energy of adsorption and a higher intrinsic reactivity of the $n$-alkane with chain length. ${ }^{44,535}$ For $n$-alkanes longer than $n-\mathrm{C}_{6}$, hydrocracking diffusion limitations are set in ref 465 and the reactivity becomes abnormally nonmonotonic. From $n$ - $\mathrm{C}_{6}$ to $n-\mathrm{C}_{8}$, reactivity decreases. Figure 48 shows the chain length dependence of the diffusion coefficient of the $n$ alkanes in ERI. It suggests that diffusion limitations are so severe that they reduce the reactivity from $n-\mathrm{C}_{6}$ to $n-\mathrm{C}_{8} .{ }^{465}$

Surprisingly, Figure 64 shows that the monotonic decrease in reactivity with $n$-alkane chain length in ERI-type zeolites is interrupted at $n-\mathrm{C}_{10}$, for $n$ - $\mathrm{C}_{10}$ is more reactive than $n$ - $\mathrm{C}_{8}{ }^{465}$ Figure 48 shows that this increase nicely correlates with an increase in diffusion rate, ${ }^{194}$ suggesting that the strong diffusion limitations remain the dominant cause for changes in reactivity from $n-\mathrm{C}_{6}$ to $n-\mathrm{C}_{10}$. Clearly, molecular simulations fully support the traditional model, which postulates that diffusion rates are the cause for both the decrease and the increase in reactivity. For $n$-alkanes longer than $n$ - $\mathrm{C}_{10}$, the reactivity as a function of $n$-alkane length changes direction again and now decreases monotonically with $n$-alkane length. This cannot be related exclusively to changes in the diffusion rates, for both experimental and simulated diffusion data indicate that the diffusion rates are still increasing with increasing $n$-alkane length and do not peak before $n-\mathrm{C}_{12}-n-\mathrm{C}_{13} .{ }^{194}$ Accordingly, continued diffusion rate dominated reactant shape selectivity would imply a continued increase in reactivity from $n-\mathrm{C}_{8}$ to $n-\mathrm{C}_{12}-n-\mathrm{C}_{13}$. The probable cause for the monotonic decrease in reactivity with $n$-alkane feed length for $n$-alkanes longer than $n$ - $\mathrm{C}_{10}$ is adsorption kinetics (diffusion) combined with adsorption thermodynamics. In the Henry regime the simulated parameter characterizing $n$-alkane reactivity (product of Henry constant and diffusion coefficient) reproduces the experimental reactivity remarkable well. ${ }^{504}$

\subsubsection{Product Shape Selectivity}

An example of the preferential production of products that combine the highest free energy of adsorption with the lowest free energy barrier to diffusion (product shape selectivity) is the product isomer distribution observed in $n$-alkane hydroconversion on TON-type zeolites. Experimental ${ }^{482}$ and simulated adsorption data ${ }^{18,172,190,356,490,491,536}$ agree that both linear and monobranched alkanes can fully adsorb into TON-type zeolite pores. Experimental ${ }^{494}$ and simulation ${ }^{190}$ methods also agree that dibranched alkanes with geminal dimethyl groups cannot adsorb in TON-type zeolite pores. By extension, dimethylalkanes should adsorb into TON-type pores as well, provided that the methyl groups are far enough apart. However, closer scrutiny reveals that dimethylalkanes can be divided into two groups: those that are commensurate with the periodicity of the TON-type zeolite wall and those that are incommensurate. The commensurate dimethylalkanes combine a low free energy of adsorption with a high free energy barrier to diffusion, whereas the incommensurate molecules combine a high free energy of adsorption with a low free energy barrier to diffusion. ${ }^{381}$ Therefore, only the latter type of dimethylalkanes are found in the hydrocracking product slate. ${ }^{381,537}$ This nice example of the importance of the Frenkel-Kontorowa effect (see section 6.6.0) to catalysis is still a subject of debate. ${ }^{381,462}$ Interestingly, the team that published the initial isomer product slates that point to a Frenkel-Kontorowa effect later published different product slates, suggesting that the experimental conditions can markedly affect the product slates.

\subsubsection{Free Energies and Diffusion: Partial Adsorption}

In catalytic cracking (i.e., cracking in the absence of added hydrogen), alkenes are formed, ${ }^{538,539}$ but the coverage of the acid sites by alkenes is extremely low, so that competitive adsorption by alkenes is negligible. ${ }^{472,476}$ Consequently, the acid sites on (nonshape selective) amorphous aluminosilicates do not release alkenes until they are hexane isomers or smaller. ${ }^{538,539}$ Once long alkanes have been cracked that far, the resulting fragments have a high enough free energy of adsorption to be replaced by the longer, unprocessed alkanes and alkenes in the feed. ${ }^{538,539}$ Figure 65 shows a representative product slate for the nonshape selective catalytic cracking of $n-\mathrm{C}_{16}{ }^{539}$ It only has a single maximum. In marked contrast to this type of a product distribution with only a single maximum, ${ }^{538}$ the catalytic cracking of long $n$-alkanes on ERI-type zeolites shows two (see Figure 66) or even four maxima (see Figure 67). ${ }^{359}$ The strong adsorption of alkenes on acid sites notwithstanding, ${ }^{475,476,538,539} n-\mathrm{C}_{22}$ and $n-\mathrm{C}_{23}$ 


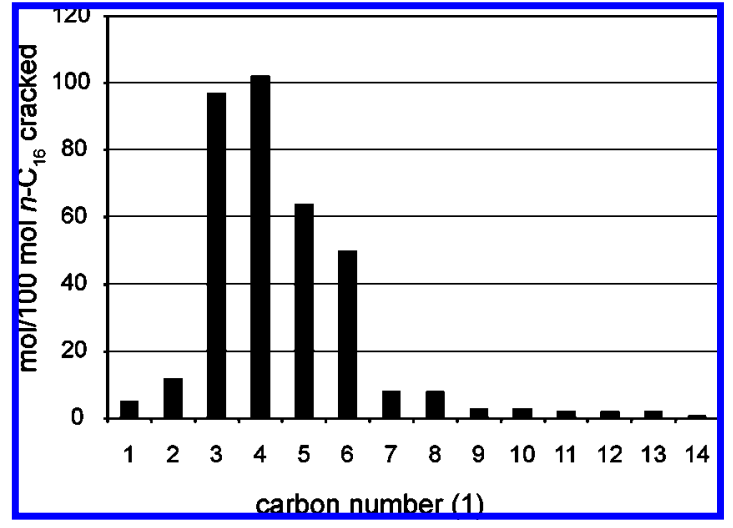

Figure 65. $n$-Hexadecane cracking in the absence of added hydrogen at $500{ }^{\circ} \mathrm{C}$ and a contact time so as to obtain $24 \% n-\mathrm{C}_{16}$ conversion over a strongly acidic silica-zirconia-alumina (UOP type B) catalytic cracking catalyst. Typical for the "packman" catalytic cracking reaction in the absence of shape selectivity, the activated alkane remains adsorbed and continues to crack until it has turned into truly tiny fragments. ${ }^{442}$

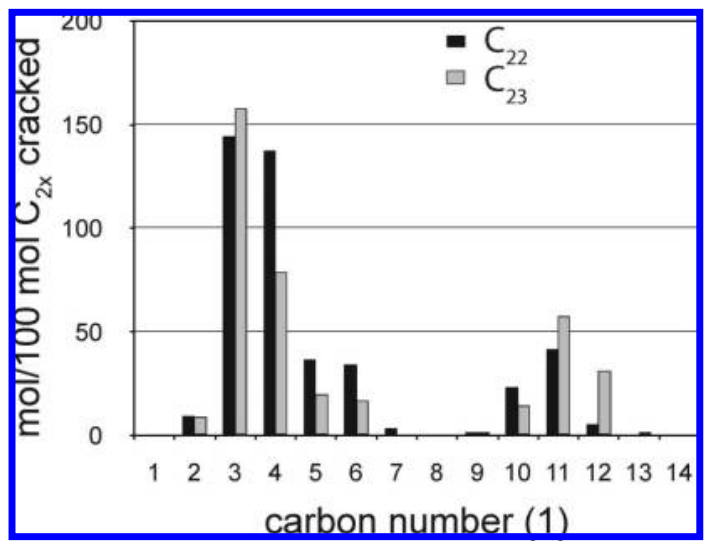

Figure 66. $n$-Docosane $\left(n-\mathrm{C}_{22}\right)$ and $n$-tricosane $\left(n-\mathrm{C}_{23}\right)$ cracking in the absence of added hydrogen at $340{ }^{\circ} \mathrm{C}$ and a contact time so as to obtain between 5 and $30 \% n$-alkane conversion over an ERItype zeolite catalytic cracking catalyst. ${ }^{357}$ As opposed to the monomodal product distribution obtained through nonselective cracking, ERI-type zeolites yield a bimodal product distribution, indicating that selected long alkane moieties escape from the usually more complete cracking process (cf. Figure 65).

selectively release $\mathrm{C}_{10}-\mathrm{C}_{12}$ moieties intact (see Figure 66), ${ }^{359}$ whereas $n-\mathrm{C}_{34}$ releases fragments as long as $\mathrm{C}_{24}-\mathrm{C}_{26}$ and $\mathrm{C}_{15}-\mathrm{C}_{17}$ intact (Figure 67). Figure 21 shows the calculated variation of Henry coefficients with chain length. The Henry coefficient for these long chain hydrocarbons is so low that the complete $n$ - $\mathrm{C}_{36}$ adsorption can be categorically excluded. Complete $n-\mathrm{C}_{36}$ adsorption has an extremely low Henry coefficient, which is equivalent to a highly positive free energy of adsorption. Accordingly, a reasonable explanation for the intact release of $\mathrm{C}_{10}-\mathrm{C}_{12}, \mathrm{C}_{15}-\mathrm{C}_{17}$, and $\mathrm{C}_{24}-\mathrm{C}_{26}$ is that these fragments were never adsorbed to begin with. The long $n$-alkane feed molecules were adsorbed only partially in the surface pockets created by ERI-type cages at the outer crystal surface. Full adsorption by more than 12 carbon atoms is unlikely. The adsorbed $\mathrm{C}_{10}-\mathrm{C}_{12}$ part is chopped off, and the nonadsorbed part is released. The released parts are immediately in the gas phase and end up in the product slate or undergo further scission reactions.

When molecular simulations indicated that $n$-alkanes as long as $n$ - $\mathrm{C}_{36}$ cannot penetrate fully into ERI-type zeolite crystals, a logical step was to assume that $n$ - $\mathrm{C}_{36}$ 's unusual cracking to product slate is a result of partial penetration into ERI-type cages at the exterior crystal surface. ${ }^{191}$ Naturally, this model is in need of experimental verification.

\subsection{In Silico Screening}

As illustrated in the preceding section, computer simulations are now at a stage where they can accurately quantify the free-energy landscape imposed by a given zeolite topology on a reacting system and thus help us to develop mechanistic explanations for why a reaction of interest yields the product distribution that is experimentally observed. But a more challenging question is whether this simulation-based methodology has predictive power; that is, whether it can screen zeolite structures to identify those particularly well suited for new applications. A case in point is hydrodewaxing, an important refining process that converts the longest hydrocarbons present in a fuel or lubricant feed into smaller molecules and thus eliminates the risk of wax precipitation and associated engine problems during later use. In hydrodewaxing, the zeolite catalyst thus needs to convert the longest hydrocarbons while leaving shorter hydrocarbons unharmed. Expressed in terms of free energies of formation, the zeolite should have a topology that maximizes the difference between the free energies of formation of the molecule to be converted, say $n-\mathrm{C}_{25}$, and a reference molecule that needs to remain untouched, such as $n-\mathrm{C}_{10}$.

The result of such "screening by computer" (summarized in Figure 68) shows that the optimally performing zeolites are ZSM-48, MTW, GON, SFE, and OFF. These zeolites all have pores with a typical tubular character and pore diameters that are optimum for absorbing the long wax molecules that need to be converted during hydrodewaxing. The use of zeolites SFE and OFF in this context has been explored ${ }^{533,540}$ but not pursued, owing to practical difficulties with synthesis, whereas ZSM-48 and MTW are at the heart of intellectual property activity ${ }^{541-543}$ on hydrodewaxing applications. Interestingly, GON had not yet been considered in this context before the computer screening, but a patent application has now been filed for a dewaxing process based on GON. ${ }^{544}$ Similarly, computer screening also identified STI for dewaxing. ${ }^{545}$ That patent applications can be entirely based on molecular simulations illustrates the considerable progress that has been made in this field.

\section{Outlook}

In the review we have discussed adsorption, diffusion, and shape selectivity. From a theoretical point of view, one can argue that for adsorption and diffusion we have a solid theoretical understanding. The catalytic section, however, is still mainly addressing concepts and is theoretically less satisfying; we are not yet in a position to obtain a similar level of quantitative predictions for shape selectivity as we have for adsorption and diffusion. For example, the success in describing shape selectivity is limited to those systems for which it is reasonable to assume internal equilibrium. Clearly, it would be desirable to develop methods to adequately integrate diffusion, adsorption, and the reaction kinetics.

Molecular simulations can be deceptive, in particular if they are successful. One would almost assume that the only true zeolites are those that form ideal infinitely large, perfect crystals without any defects. It is a fact that the gap between virtual reality and real reality is large and, in most, if not all, practical applications, zeolites are far less ideal than 


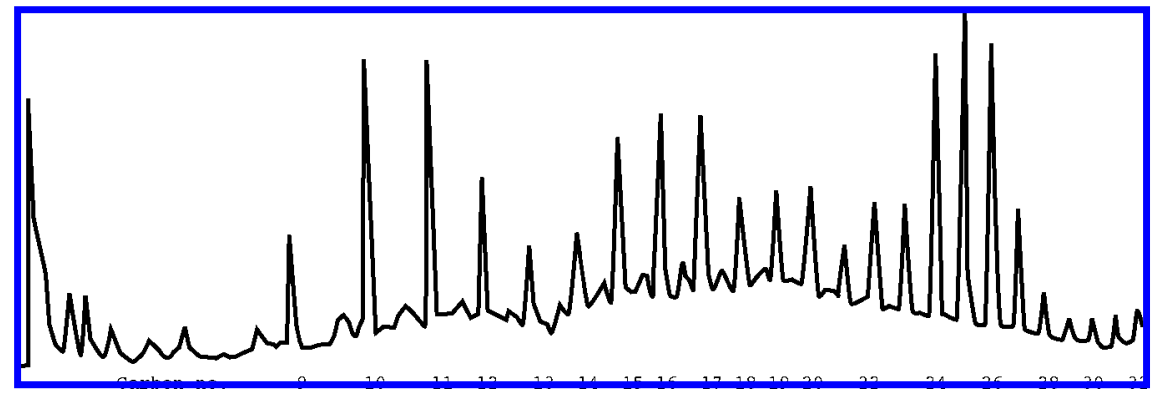

Figure 67. Chromatogram of the liquid products collected from $n$-hexatriacontane $\left(n-C_{34}\right)$ cracking in the absence of added hydrogen at $400{ }^{\circ} \mathrm{C}$ over an ERI-type zeolite catalytic cracking catalyst. ${ }^{359}$ The numbers underneath the chromatogram indicate the carbon number of the alkane eluting from the column. As opposed to the monomodal product distribution obtained through nonselective cracking, ERI-type zeolites yield a trimodal product distribution, indicating that selected long alkane moieties escape from the usually more complete cracking process (cf. Figure 65). Figure based on data from ref 504

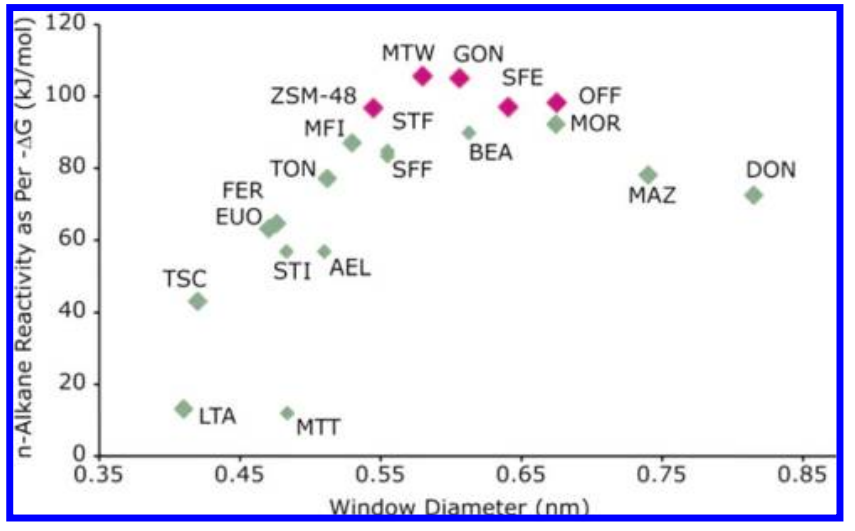

Figure 68. Zeolite screening by computer. For hydrodewaxing, the optimal reactivity of a structure can be expressed in terms of the free energy of adsorption $\Delta G$ of a long $n$-alkane $\left(n-\mathrm{C}_{25}\right)$. Frequently, zeolites are characterized by their window diameter, that is, the smallest diameter molecules "see" when moving through the material. Topologies that exhibit a highly negative value preferentially convert long instead of short $n$-alkanes; that is, they optimally remove the waxes and leave the shorter hydrocarbons. Indirect experimental evidence for the validity of this thermodynamic approach is the match between the optimum structure and the focus of a flurry in patenting activity. The topologies in red have been patented by Shell ${ }^{541}$ and ExxonMobil, ${ }^{542}$ and GON has been "discovered" by simulations. ${ }^{544}$ (Figure reproduced with permission from ref 503).

assumed in the simulations. Nevertheless, we strongly feel that accurate and reliable predictions on the thermodynamics and transport properties of an ideal, but well defined, reference system are often a very useful starting point. The main conclusion of this review is that molecular simulations have progressed to the extent that for hydrocarbons reliable predictions can be obtained through simulating these "ideal" reference systems.

Of course, one can systematically investigate less ideal zeolites. Difficulties or challenges have already been seen in the case in which we replaced Si by Al. Whereas for all silica structures the complete crystal structure is know, as soon as we have $\mathrm{Al}$ atoms, the experimental data are not providing a unique structure and the molecular simulations are far less convincing. In fact, before one can study adsorption or diffusion, one does need to develop a molecular model of the structure of these nonideal zeolites.

Among all possible nonidealities, a question which is almost completely ignored in this review is how do the molecules enter into the zeolite? All but a very few simulations assume infinitely large crystals. That molecules have to enter from the gas phase is not occurring, as these ideal crystals do not have an interface. Yet, from a practical point of view, the gas-zeolite interface can be very important. For example, some reaction mechanisms are proposed in which shape selectivity is induced by the exterior of the zeolite. ${ }^{537}$ Or, the difference in diffusion coefficients as measured by uptake experiments and NMR experiments is often attributed to a surface barrier at the zeolite-gas interface. ${ }^{252}$ Another important and related question is how much of a zeolite crystal participates in a reaction? From a simulation point of view, these are extremely interesting questions to study (see refs 546 and 414 for some interesting simulations). For example, one could determine the free energy profiles of molecules entering the zeolite as a function of the chemical potential of the adsorbed gas. The computational methods are in place to address such a question, but the challenge is to develop a reliable model for the surface of a zeolite crystal.

During the writing of this review, the price of the main feedstock of hydrocarbons has increased to levels that were assumed impossible only a few years ago. This gives an additional incentive to ensure that current processes are as energy efficient as possible. The underlying trends also imply an irreversible shift toward heavier feeds which are dominated by asphaltene types of molecules. Our current knowledge is based on the typical light feeds that we are considering in this review. It would be interesting to see whether for these very complex feeds molecular simulations can contribute to a better understanding of conversion processes at a molecular level. Clearly, this requires new algorithms that efficiently can simulate these types of molecules.

The approximately 180 zeolite structures known to exist constitute only of a very small fraction of the more than $2,500,000$ structures that are feasible on theoretical grounds. ${ }^{547}$ A database of hypothetical zeolite structures has been regarded as an important step toward "designer catalysts". ${ }^{488}$ It can in principle be screened for zeolites that are suitable for particular applications using the same methodology that is described in this review to screen existing zeolites. To cope with a large number of structures and to identify efficiently those with useful and superior catalytic properties will clearly involve new computational approaches.

Clearly, it would be very exciting to effectively screen all 2,500,000 hypothetical zeolites and find one with superior performance. Any such in silico promise can, however, only be realized if it is also possible to synthesize the identified structures. This brings us to one of the key problems in zeolite synthesis: to understand how one can form a particular zeolite structure. From an energetic point of view, all zeolites 
have similar energies, and how can we understand a desired structure will form. This requires detailed insights into the nucleation and crystal growth of zeolites. ${ }^{40,549}$ Molecular simulation might provide insights that help us to rationally control and guide the synthesis processes toward desired new zeolite structures.

\section{Acknowledgments}

The parts of this review that cannot be found in the literature we learned from our co-workers: Edith Beerdsen, Sofia Calero, David Dubbeldam, Daan Frenkel, Sami Karaborni, R. Krishna, Bei Liu, Merijn Schenk, Ilja Siepmann, Thijs Vlugt, and Nils Zimmerman. Special thanks go to Alex Bell, R. Krishna, Joe Swisher, and Jo Tingsanchali for a critical reading of the manuscript. This work has been partially supported by the EC through Marie Curie EXT project MEXT-CT-2005-023311.

\section{References}

(1) Baerlocher, C.; Meier, W.; Olson, D. Atlas of zeolite framework types; Elsevier: Amsterdam, 2001.

(2) Auerbach, S. M., Carrado, K., Dutta, P., Eds.; Handbook of Zeolite Science and Technology; Marcel Dekker: New York, 2004.

(3) Beerdsen, E.; Dubbeldam, D.; Smit, B. J. Phvs. Chem. B 2006, 110, 22754.

(4) Barrer, R. Zeolites and clay minerals as sorbents and molecular sieves; Academic Press: London, 1978.

(5) Thomas, J. Sci. Am. 1992, 266, 82

(6) Corma, A. Chem. Rev. 1997, 97, 2373.

(7) Corma, A. Chem. Rev 1995, 95, 559.

(8) Jensen, R. H. Refining processes: setting the scene. In Zeolites for Cleaner Technologies; Guisnet, M., Gilson, J.-P., Eds.; Imperial College Press: London, 2002; Vol. 3.

(9) Catlow, C. R. A. Modelling of Structure and Reactivity in Zeolites; Academic Press: London, 1992.

(10) Catlow, C. R. A., van Santen, R. A., Smit, B., Eds.; Computer modeling of micorporous materials; Elsevier: Amsterdam, 2004.

(11) Allen, M.; Tildesley, D. Computer Simulation of Liquids; Clarendon Press: Oxford, 1987.

(12) Frenkel, D.; Smit, B. Understanding Molecular Simulations: from Algorithms to Applications, 2nd ed.; Academic Press: San Diego, 2002.

(13) Haile, J. Molecular Dynamics Simulations: Elementary Methods; John Wiley and Sons: New York, 1992.

(14) Rapaport, D. The Art of Molecular Dynamics Simulation; Cambridge University Press: New York, 1995.

(15) Andersen, H. J. Chem. Phvs. 1980, 72, 2384

(16) Nosé, S. J. Chem. Phvs. 1984, 81, 511.

(17) Hoover, W. Phvs. Rev. A 1985, 31, 1695.

(18) Webb, E. B. I.; Grest, G.; Mondello, M. J.Phvs. Chem. B 1999, $103,4949$.

(19) Jakobtorweihen, S.; Verbeek, M. G.; Lowe, C. P.; Keil, F. J.; Smit, B. Phys. Rev. Lett. 2005, 95, 044501.

(20) Panagiotopoulos, A. Mol. Phvs. 1987, 61, 813.

(21) Panagiotopoulos, A. Mol. Phvs. 1987, 62, 701.

(22) Liu, S. S.; Yang, X. N. J. Chem. Phvs. 2006, 124, 244705.

(23) Jiang, J.; Sandler, S. I. Phvs. Rev. B 2003, 68, 245412.

(24) Widom, B. J. Phys. Chem. 1982, 86, 869.

(25) Fichthorn, K. A.; Weinberg, W. H. J. Chem. Phvs. 1991, 95, 1090

(26) Snyder, M. A.; Vlachos, D. G. J. Chem. Phos. 2005, 123, 184707.

(27) Krishna, R.; van Baten, J. M. Chem. Eng. Technol. 2005, 28, 160.

(28) Keil, F. J.; Krishna, R.; Coppens, M. O. Rev. Chem. Eng. 2000, 16, 71.

(29) Auerbach, S. M.; Henson, N. J.; Cheetham, A. K.; Metiu, H. I. J. Phvs. Chem. 1995, 99, 10600.

(30) Auerbach, S. M. Int. Rev. Phys. Chem. 2000, 19, 155.

(31) Campana, L.; Selloni, A.; Weber, J.; Pasquarello, A.; Papai, I.; Goursot, A. Chem. Phys. Lett. 1994, 226, 245.

(32) Rozanska, X.; van Santen, R. A.; Demuth, T.; Hutschka, F.; Hafner, J. J. Phys. Chem. B 2003, 107, 1309.

(33) Benco, L.; Demuth, T.; Hutschka, F. Comput. Mater. Sci. 2003, 27, 87.

(34) Nusterer, E.; Blochl, P. E.; Schwarz, K. Angew. Chem., Int. Ed. Engl. 1996, 35,175 .

(35) Fois, E.; Gamba, A.; Tilocca, A. J. Phvs. Chem. B 2002, 106, 4806.
(36) Benco, L.; Demuth, T.; Hafner, J.; Hutschka, F.; Toulhoat, H. J. Chem. Phvs. 2001, 114, 6327.

(37) Benco, L.; Demuth, T.; Hafner, J.; Hutschka, E.; Toulhoat, H. $\underline{J}$. Catal. 2002, 205, 147.

(38) Haase, F.; Sauer, J. Microporous Mesoporous Mater 2000, 35-36, 379.

(39) Chiu, M. E.; Slater, B.; Gale, J. D. Angew. Chem., Int. Ed. 2005, 44, 1213.

(40) Auerbach, S. M.; Ford, M. H.; Monson, P. A. Curr. Opin. Colloid Interface Sci. 2005, 10, 220.

(41) Sherwood, P.; et al. THEOCHEM 2003, 632, 1.

(42) Sierka, M.; Sauer, J. J. Chem. Phys. 2000, 112, 6983.

(43) Clark, L. A.; Sierka, M.; Sauer, J. J. Am. Chem. Soc. 2003, 125, 2136.

(44) Clark, L. A.; Sierka, M.; Sauer, J. J. Am. Chem. Soc. 2004, 126, 936.

(45) Nieminen, V.; Sierka, M.; Murzin, D. Y.; Sauer, J. J. Catal. 2005, $231,393$.

(46) Deka, R. C.; Nasluzov, V. A.; Shor, E. A. I.; Shor, A. M.; Vayssilov, G. N.; Rosch, N. J. Phvs. Chem. B 2005, 109, 24304.

(47) June, R.; Bell, A.; Theodorou, D. J. Phvs. Chem. 1992, 96, 1051.

(48) Runnebaum, R.; Maginn, E. J. Phvs. Chem. B 1997, 101, 6394.

(49) Schuring, D.; Jansen, A. P. J.; van Santen, R. A. J.Phvs. Chem. B 2000, 104, 941

(50) Vlugt, T.; Krishna, R.; Smit, B. J. Phys. Chem. B 1999, 103, 1102.

(51) Smit, B.; Loyens, L.; Verbist, G. Faradav Discuss. 1997, 106, 93.

(52) Harris, J.; Rice, S. J. Chem. Phvs. 1988, 89, 5898.

(53) Siepmann, J.; Frenkel, D. Mol. Phys. 1992, 75, 59.

(54) Frenkel, D.; Mooij, G.; Smit, B. J.Phvs.: Condens. Matter 1992, 4, 3053.

(55) Rosenbluth, M.; Rosenbluth, A. J. Chem. Phvs. 1955, 23, 356.

(56) Dijkstra, M. J. Chem. Phvs. 1997, 107, 3277.

(57) Martin, M.; Siepmann, J. I. J. Phvs. Chem. B 1999, 103, 4508.

(58) Deem, M.; Bader, J. Mol. Phvs. 1996, 87, 1245.

(59) Wu, M.; Deem, M. Mol. Phvs. 1999, 97, 559.

(60) Chen, Z.; Escobedo, F. J. Chem. Phvs. 2000, 113, 11382.

(61) Wick, C.; Siepmann, J. I. Macromolecules 2000, 33, 7207.

(62) Chen, B.; Siepmann, J. I. J. Phvs. Chem. B 1999, 103, 5370.

(63) Macedonia, M.; Maginn, E. Mol. Phvs. 1999, 96, 1375.

(64) Vlugt, T.; Martin, M.; Smit, B.; Siepmann, J. I.; Krishna, R. Mol. Phvs. 1998, 94, 727.

(65) Maginn, E.; Bell, A.; Theodorou, D. J. Phvs. Chem. 1995, 99, 2057.

(66) Bennett, C. Molecular dynamics and transition state theory: The simulation of infrequent events. In Algorithms for chemical computations; Christoffersen, R., Ed.; ACS Symposium Series; American Chemical Society: Washington, DC, 1977.

(67) Chandler, D. J. Chem. Phvs. 1978, 68, 2959.

(68) Sevick, E.; Bell, A. T.; Theodorou, D. N. J. Chem. Phvs. 1993, 98, 3196.

(69) Ruiz-Montero, M.; Frenkel, D.; Brey, J. Mol. Phvs. 1997, 90, 925.

(70) Bolhuis, P.; Dellago, C.; Chandler, D. Faradav Discuss. 1998, 110, 421.

(71) Dellago, C.; Bolhuis, P.; Csajka, F.; Chandler, D. J. Chem. Phvs. 1998, 108,1964

(72) Dellago, C.; Bolhuis, P.; Chandler, D. J. Chem. Phvs. 1999, 110, 6617.

(73) Structure Commission of the International Zeolite Association, http://www.iza-structure.org/databases/.

(74) Bezus, A.; Kiselev, A.; Lopatkin, A.; Du, P. J. Chem. Soc., Faradav Trans. 2 1978, 74, 367

(75) Thomson, K. T.; McCormick, A. V.; Davis, H. T. $\underline{\text { J. Chem. Phvs }}$ 2000, 112, 3345 .

(76) Demontis, P.; Suffritti, G. Chem. Rev. 1997, 97, 2845.

(77) Ryckaert, J. P.; McDonald, I. R.; Klein, M. L. Mol. Phvs. 1989, 67, 957.

(78) Smit, B.; Karaborni, S.; Siepmann, J. I. J. Chem. Phys. 1995, 102, 2126.

(79) Siepmann, J. I.; Karaborni, S.; Smit, B. J. Am. Chem. Soc. 1993, 115,6454

(80) Siepmann, J. I.; Karaborni, S.; Smit, B. Nature 1993, 365, 330.

(81) Chen, B.; Potoff, J.; Siepmann, J. I. J. Phys. Chem. B 2001, 105, 3093.

(82) Martin, M.; Siepmann, J. I. J. Phvs. Chem. B 1998, 102, 2569.

(83) Nath, S.; Escobedo, F.; Pablo, J. d. J. Chem. Phvs. 1998, 108, 9905.

(84) Siepmann, J. I.; Martin, M.; Mundy, C.; Klein, M. Mol. Phvs. 1997, 90, 687.

(85) Smit, B.; Karaborni, S.; Siepmann, J. I. J. Chem. Phys. 1998, 109, 352.

(86) Wick, C.; Martin, M.; Siepmann, J. J. Phvs. Chem. B 2000, 104, 8008 .

(87) De Luca, G.; Arbouznikov, A.; Goursot, A.; Pullumbi, P. J. Phys. Chem. B 2001, 105, 4663.

(88) Tielens, F.; Geerlings, P. Chem. Phvs. Lett. 2002, 354, 474. 
(89) Ghorai, P. K.; Sluiter, M.; Yashonath, S.; Kawazoe, Y. J. Am. Chem. Soc. 2003, 125, 16192.

(90) Smit, B. J. Phys. Chem. 1995, 99, 5597

(91) Goodbody, S.; Watanabe, K.; MacGowan, D.; Walton, J.; Quirke, N. J. Chem. Soc., Faradav Trans. 1991, 87, 1951.

(92) Hufton, J. J. Phvs. Chem. 1991, 95, 8836.

(93) Demontis, P.; Suffritti, G.; Fois, E.; Quartieri, S. J. Phys. Chem. 1992, 96, 1482.

(94) Dubbeldam, D.; Calero, S.; Vlugt, T. J. H.; Krishna, R.; Maesen, T.; Smit, B. J. Phys. Chem. B 2004, 108, 12301.

(95) Pascual, P.; Ungerer, P.; Tavitian, B.; Pernot, P.; Boutin, A. Phvs. Chem. Chem. Phys. 2003, 5, 3684.

(96) Pascual, P.; Ungerer, P.; Tavitian, B.; Boutin, A. J. Phys. Chem. B 2004, 108, 393

(97) Dubbeldam, D.; Calero, S.; Vlugt, T. J. H.; Krishna, R.; Maesen, T.; Beerdsen, E.; Smit, B. Phys. Rev. Lett. 2004, 93, art no. 088302.

(98) Jakobtorweihen, S.; Hansen, N.; Keil, F. J. Mol. Phys. 2005, 103, 471.

(99) Liu, B.; Smit, B.; Rey, F.; Valencia, S.; Calero, S. J. Phys. Chem. C. 2008, 112, 2492.

(100) Loisruangsin, A.; Fritzsche, S.; Hannongbua, S. Chem. Phys. 2008, 344, 1 .

(101) Lwenstein, W. Am. Mineral. 1954, 39, 92

(102) Dempsey, E.; Kuehl, G. H.; Olson, D. J. Phys. Chem. 1969, 73, 387.

(103) Melchior, M.; Vaughan, D.; Jacobson, A. J. Am. Chem. Soc. 1982, 104,4859

(104) Den Ouden, C.; Jackson, R.; Catlow, C. Zeolites 1992, 12, 870.

(105) Schroeder, K. P.; Sauer, J. J. Phys. Chem. 1993, 97, 6579.

(106) Slaughter, M.; Yu, J.-Y. Binding Energies and Structural Properties of the Heulandite-Clinoptilolite Series. In Zeolite '93: 4th International Conference on the Occurrence, Properties, and Utilization of Natural Zeolites; Ming, D. W., Mumpton, F. A., Eds.; International Committee on Natural Zeolites: Brockport, New York, 1995.

(107) Tarling, S.; Barnes, P. Acta Crystallogr. 1988, B44, 128.

(108) Fyfe, C.; Gobbi, G.; Klinowski, J.; Thomas, J.; Ramdas, S. Nature 1982, 296, 530

(109) Haag, W.; Lago, R.; Weisz, P. Nature 1984, 304, 589.

(110) Han, O. H.; Kim, C. S.; Hong, S. B. Angew. Chem., Int. Ed. 2002, $41,469$.

(111) Smit, B.; Denouden, C. J. J. J. Phys. Chem. 1988, 92, 7169.

(112) Auerbach, S. M.; Bull, L. M.; Henson, N. J.; Metiu, H. I.; Cheetham, A. K. J. Phys. Chem. 1996, 100, 5923.

(113) Lachet, V.; Buttefey, S.; Boutin, A.; Fuchs, A. H. Phys. Chem. Chem. Phys. 2001, 3, 80

(114) Buttefey, S.; Boutin, A.; Mellot-Draznieks, C.; Fuchs, A. H. J. Phys. Chem. B 2001, 105, 9569.

(115) Buttefey, S.; Boutin, A.; Fuchs, A. H. Mol. Simul. 2002, 28, 1049

(116) Jaramillo, E.; Auerbach, S. M. J. Phvs. Chem. B 1999, 103, 9589.

(117) Beerdsen, E.; Smit, B.; Calero, S. J. Phys. Chem. B 2002, 106, 10659.

(118) Macedonia, M. D.; Moore, D. D.; Maginn, E. J.; Olken, M. M. Langmuir 2000, 16, 3823.

(119) Mellot-Draznieks, C.; Buttefey, S.; Boutin, A.; Fuchs, A. H. Chem. Commun. 2001, 21, 2200.

(120) Vitale, G.; Mellot, C. F.; Bull, L. M.; Cheetham, A. K. J. Phys. Chem. B 1997, 101, 4559 .

(121) Calero, S.; Dubbeldam, D.; Krishna, R.; Smit, B.; Vlugt, T. J. H.; Denayer, J. F.; Martens, J. A.; Maesen, T. L. M. J. Am. Chem. Soc. 2004, 126, 11377.

(122) Garcia-Perez, E.; Dubbeldam, D.; Liu, B.; Smit, B.; Calero, S. Angew. Chem., Int. Ed. 2007, 46, 276.

(123) Liu, B.; Garcia-Perez, E.; Dubbeldam, D.; Smit, B.; Calero, S. J. Phys. Chem. C 2007, 111, 10419.

(124) Sastre, G.; Fornes, V.; Corma, A. J. Phys. Chem. B 2002, 106, 701.

(125) Macedonia, M.; Maginn, E. AIChEJ. 2000, 46, 2504.

(126) Liu, B.; Smit, B. Phvs. Chem. Chem. Phvs. 2006, 8, 1852.

(127) Calero, S.; Lobato, M.; Garcia-Perez, E.; Mejias, J.; Lago, S.; Vlugt, T.; Maesen, T.; Smit, B.; Dubbeldam, D. J. Phys. Chem. B 2006, 110,5838

(128) Ruthven, D. Principles of Adsorption and Adsorption Processes; Wiley-Interscience: New York, 1984.

(129) Talu, O. Adv. Colloid Interface Sci. 1998, 77, 227

(130) Haag, W. Stud. Surf. Sci. Catal. 1994, 84, 1375.

(131) Krishna, R.; Wesselingh, J. Chem. Eng. Sci. 1997, 52, 861

(132) Gregg, S.; Sing, K. Adsorption, Surface Area and Porosity; Academic Press: London, 1982.

(133) Vuong, T.; Monson, P. A. Langmuir 1996, 12, 5425.

(134) Myers, A. L.; Monson, P. A. Langmuir 2002, 18, 10261.

(135) Hill, T. Adv. Catal. 1952, 4, 211.

(136) Maesen, T.; Krishna, R.; Baten, J. M. V.; Smit, B.; Calero, S.; Castillo-Sanchez, J. M. J. Catal. 2008, 256, 95.

(137) Myers, A. L.; Prausnitz, J. AIChE J. 1965, 11, 121.

(138) Krishna, R.; Calero, S.; Smit, B. Chem. Eng. J. 2002, 88, 81.

(139) Skoulidas, A. I.; Sholl, D. S. J. Phys. Chem. B 2002, 106, 5058.
(140) Snurr, R.; June, R.; Bell, A.; Theodorou, D. Mol. Simul. 1991, 8, 73.

(141) Bouyermaouen, A.; Bellemans, A. J. Chem. Phys. 1998, 108, 2170.

(142) Vlugt, T.; Zhu, W.; Kapteijn, F.; Moulijn, J.; Smit, B.; Krishna, R. J. Am. Chem. Soc. 1998, 120, 5599.

(143) Ashtekar, A.; McLeod, S.; Mantle, M.; Bariie, P.; Gladden, L.; Hastings, J. J. Phys. Chem. B 2000, 104, 5281.

(144) Calero, S.; Smit, B.; Krishna, R. Phys. Chem. Chem. Phys. 2001, 3, 4390.

(145) Calero, S.; Smit, B.; Krishna, R. J. Catal. 2001, 202, 395.

(146) Du, Z.; Vlugt, T.; Smit, B.; Manos, G. AIChE J. 1998, 44, 1756.

(147) Gupta, A.; Clark, L. A.; Snurr, R. Q. Langmuir 2000, 16, 3910.

(148) Heuchel, M.; Snurr, R. Q.; Buss, E. Langmuir 1997, 13, 6795.

(149) June, R.; Bell, A.; Theodorou, D. J. Phys. Chem. 1990, 94, 1508.

(150) June, R.; Bell, A.; Theodorou, D. J. Phys. Chem. 1990, 94, 8232.

(151) Krishna, R.; Paschek, D. Phys. Chem. Chem. Phys. 2001, 3, 453.

(152) Krishna, R.; Smit, B.; Calero, S. Chem. Soc. Rev. 2002, 31, 185.

(153) Krishna, R.; Smit, B.; Vlugt, T. J. Phys. Chem. A 1998, 102, 7727.

(154) Macedonia, M.; Maginn, E. Fluid Phase Equilib. 1999, 160, 19.

(155) Maginn, E. J.; Bell, A. T.; Theodorou, D. N. Low-occupancy sorption thermodynamics of long alkanes in sililcalite via Molecular Simulation. In Zeolites and Related Microporous Materials: State of the Art 1994; Weitkamp, J., Karge, H., Pfeifer, H., Hlderich, W., Eds.; Elsevier: Amsterdam, 1994; Vol. 84.

(156) Maginn, E.; Bell, A.; Theodorou, D. J. Phys. Chem. 1996, 100, 7155.

(157) Nowak, A.; Ouden, C. d.; Pickett, S.; Smit, B.; Cheetham, A.; Post, M.; Thomas, J. J. Phys. Chem. 1991, 95, 848.

(158) Pickett, S.; Nowak, A.; Thomas, J.; Peterson, B.; Swift, J.; Cheetham, A.; Ouden, C. d.; Smit, B.; Post, M. J. Phys. Chem. 1990, 94, 1233.

(159) Schenk, M.; Vidal, S.; Vlugt, T.; Smit, B.; Krishna, R. Langmuir 2001, 17, 1558.

(160) Smit, B.; Siepmann, J. Science 1994, 264, 1118.

(161) Smit, B.; Siepmann, J. J. Phys. Chem. 1994, 98, 8442.

(162) Smit, B. Mol. Phys. 1995, 85, 153.

(163) Smit, B.; Maesen, T. Nature 1995, 374, 42.

(164) Nagumo, R.; Takaba, H.; Nakao, S. J. Phys. Chem. B 2003, 107, 14422.

(165) Fox, J. P.; Rooy, V.; Bates, S. P. Microporous Mesoporous Mater. 2004, 69, 9 .

(166) Fuchs, A.; Cheetham, A. J. Phys. Chem. B 2001, 105, 7375

(167) Makrodimitris, K.; Papadopoulos, G. K.; Theodorou, D. N. J. Phvs. Chem. B 2001, 105, 777.

(168) Leyssale, J. M.; Papadopoulos, G. K.; Theodorou, D. N. J. Phys. Chem. B 2006, 110, 22742.

(169) Krishna, R.; van Baten, J. M.; Garcia-Perez, E.; Calero, S. Chem. Phys. Lett. 2006, 429, 219.

(170) Desbiens, N.; Boutin, A.; Demachy, I. J. Phys. Chem. B 2005, 109, 24071.

(171) Ramachandran, C. E.; Chempath, S.; Broadbelt, L. J.; Snurr, R. Q. Microporous Mesoporous Mater. 2006, 90, 293.

(172) Demuth, T.; Rozanska, X.; Benco, L.; Hafner, J.; van Santen, R. A.; Toulhoat, H. J. Catal. 2003, 214, 68.

(173) Sun, M.; Shah, D.; Xu, H.; Talu, O. J. Phys. Chem. 1998, 102, 1466.

(174) Sun, M.; Talu, O.; Shah, D. J. Phys. Chem. 1996, 100, 17276.

(175) Eder, F. Thermodynamics and siting of alkane sorption in molecular sieves. Ph.D. Thesis, University of Twente, 1996.

(176) Zhu, W.; Kapteijn, F.; Moulijn, J. A. Phvs. Chem. Chem. Phys. 2000, 2, 1989.

(177) Cavalcante, C., Jr.; Ruthven, D. Ind. Eng. Chem. Rev. 1995, 34, 177.

(178) Dubinin, M.; Rakhmatkariev, G.; Isirikyan, A. Izv. Akad. Nauk SSSR, Ser. Khim. 1989, 10, 2333.

(179) Rakhmatkariev, G.; Zhalalov, K.; Akhmedov, K. Uzb. Khim. Zh. 1988, 3, 68

(180) Micke, A.; Bülow, M.; Kocirik, M.; Struve, P. J. Chem. Phys. 1994, 98, 12337.

(181) Zhu, W.; Kapteijn, F.; van der Linden, B.; Moulijn, J. A. Phys. Chem. Chem. Phys. 2001, 3, 1755.

(182) Yang, Y.; Rees, L. Microporous Mater. 1997, 12, 117.

(183) Sun, W. J.; TristramNagle, S.; Suter, R. M.; Nagle, J. F. Proc. Natl. Acad. Sci. U.S.A. 1996, 93, 7008.

(184) Well, W. v.; Wolthuizen, J.; Smit, B.; Hooff, J. v.; Santen, R. v. Angew. Chem. Int. Ed. 1995, 34, 2543.

(185) Millot, B.; Methivier, A.; Jobic, H. J. Phys. Chem. B 1998, 102, 3210 .

(186) Makowski, W.; Majda, D. Appl. Surf. Sci. 2005, 252, 707.

(187) Makowski, W.; Majda, D. J. Porous Mat. 2007, 14, 27.

(188) Morell, H.; Angermund, K.; Lewis, A. R.; Brouwer, D. H.; Fyfe, C. A.; Gies, H. Chem. Mater. 2002, 14, 2192.

(189) Floquet, N.; Coulomb, J. P.; Bellat, J. P.; Simon, J. M.; Weber, G.; Andre, G. J. Phys. Chem. C 2007, 111, 18182.

(190) Maesen, T.; Schenk, M.; Vlugt, T. J. H.; Jonge, J. d.; Smit, B. J. Catal. 1999, 188, 403. 
(191) Dubbeldam, D.; Calero, S.; Maesen, T. L. M.; Smit, B. Angew. Chem.. Int. Ed. 2003, 42, 3624.

(192) Dubbeldam, D.; Smit, B. J. Phvs. Chem. B 2003, 107, 12138.

(193) Krishna, R.; van Baten, J. M. Sep. Purif. Technol. 2008, 60, 315.

(194) Dubbeldam, D.; Calero, S.; Maesen, T. L. M.; Smit, B. Phvs. Rev. Lett. 2003, 90, art. no. 245901

(195) Tepper, H. L.; Hoogenboom, J. P.; van der Vegt, N. F. A.; Briels, W. J. J. Chem. Phys. 1999, 110, 11511.

(196) Krishna, R.; van Baten, J. M. Chem. Eng. Technol. 2006, 29, 1429.

(197) Martin, C.; Tosi-Pellenq, N.; Patarin, J.; Coulomb, J. Langmuir 1998, $14,1774$.

(198) Lachet, V.; Boutin, A.; Pellenq, R.; Nicholson, D.; Fuchs, A. J. Phvs. Chem. 1996, 100, 9006.

(199) Maris, T.; Vlugt, T.; Smit, B. J. Phvs. Chem. B 1998, 102, 7183.

(200) Well, W. v.; Cottin, X.; Haan, J. d.; Santen, R. v.; Smit, B. Angew. Chem., Int. Ed. 1998, 37, 1081.

(201) Well, W. v.; Cottin, X.; Haan, J. d.; Smit, B.; Nivarthy, G.; Lercher, J.; Hooff, J. v.; Santen, R. v. J. Phvs. Chem. B 1998, 102, 3945.

(202) Wang, H. Y.; Turner, E. A.; Huang, Y. N. J. Phys. Chem. B 2006, 110,8240

(203) Well, W. v.; Cottin, X.; Smit, B.; Hooff, J. v.; Santen, R. v. J. Phvs. Chem. B 1998, 102, 3952.

(204) Eder, F.; Lercher, J. J. Phvs. Chem. B 1997, 101, 1273.

(205) Ndjaka, J. M. B.; Zwanenburg, G.; Smit, B.; Schenk, M. Microporous Mesoporous Mater. 2004, 68, 37.

(206) Pascual, P.; Boutin, A. Phys. Chem. Chem. Phys. 2004, 6, 2015.

(207) Liu, B.; Smit, B. J. Phvs. Chem. B 2006, 110, 20166.

(208) Liu, H.; Zhang, Z.; Chen, B. H.; Zhao, Y. J. Porous Mater. 2008, $15,119$.

(209) Guo, C.; Talu, O.; Hayhurst, D. AIChE J. 1989, 35, 573.

(210) Olsen, D.; Kokotailo, G.; Lawton, S.; Meier, W. J. Phys. Chem. 1981, $85,2238$.

(211) Koningsveld, H. v.; Jansen, J.; Bekkum, H. v. Zeolites 1987, 7, 564

(212) Snurr, R.; Bell, A.; Theodorou, D. J. Phys. Chem. 1993, 97, 13742

(213) Talu, O.; Guo, C.-J.; Hayhurst, D. J. Phys. Chem. 1989, 93, 7294.

(214) Clark, L.; Snurr, R. Chem. Phvs. Lett. 1999, 308, 155.

(215) Vlugt, T. J. H.; Schenk, M. J. Phvs. Chem. B 2002, 106, 12757.

(216) Calleja, G.; Pan, J.; Calles, J. A. J. Chem. Eng. Data 1998, 43, 994.

(217) Daems, I.; Methivier, A.; Leflaive, P.; Fuchs, A. H.; Baron, G. V.; Denayer, J. F. M. J. Am. Chem. Soc. 2005, 127, 11600.

(218) Tielens, F.; Denayer, J. F. M.; Daems, I.; Baron, G. V.; Mortier, W. J.; Geerlings, P. J. Phys. Chem. B 2003, 107, 11065.

(219) Garcia-Perez, E.; Dubbeldam, D.; Maesen, T. L. M.; Calero, S. J. Phvs. Chem. B 2006, 110, 23968.

(220) Garcia-Sanchez, A.; Garcia-Perez, E.; Dubbeldam, D.; Krishna, R.; Calero, S. Adsorpt. Sci. Technol. 2007, 25, 417.

(221) Granato, M. A.; Vlugt, T. J. H.; Rodrigues, A. E. Ind. Eng. Chem. Res. 2007, 46, 321.

(222) Wender, A.; Barreau, A.; Lefebvre, C.; Di Lella, A.; Boutin, A.; Ungerer, P.; Fuchs, A. H. Adsorpt. Sci. Technol. 2006, 24, 713.

(223) Siperstein, F. R.; Myers, A. L. AIChE J. 2001, 47, 1141

(224) Vantassel, P. R.; Davis, H. T.; McCormick, A. V. Langmuir 1994, $10,1257$.

(225) Jameson, C. J.; Jameson, A. K.; Kostikin, P.; Baello, B. I. J. Chem. Phvs. 2000, 112, 323

(226) Gergidis, L. N.; Theodorou, D. N. J. Phys. Chem. B 1999, 103, 3380.

(227) Gergidis, L. N.; Theodorou, D. N.; Jobic, H. J.Phvs. Chem. B 2000 , 104, 5541 .

(228) Schuring, D.; Koriabkina, A.; Jong, A. d.; Smit, B.; Santen, R. v. J. Phys. Chem. B 2001, 105, 7690.

(229) Adhangale, P.; Keffer, D. Langmuir 2002, 18, 10455.

(230) Dunne, L. J.; Manos, G.; Du, Z. M. Chem. Phvs. Lett. 2003, 377, 551.

(231) Denayer, J. F. M.; Ocakoglu, R.; Huybrechts, W.; Dejonckheere, B.; Jacobs, P.; Calero, S.; Krishna, R.; Smit, B.; Baron, G. V.; Martens, J. A. J. Catal. 2003, 220, 66.

(232) Zhang, Y.; Furukawa, S.; Nitta, T. J. Chem. Eng. Jpn. 2003, 36, 1085.

(233) Zhang, Y.; Furukawa, S. I.; Nitta, T. J. Chem. Eng. Jpn. 2004, 37, 563.

(234) Fox, J. P.; Bates, S. P. J. Phvs. Chem. B 2004, 108, 17136.

(235) Chempath, S.; Denayer, J. F. M.; De Meyer, K. M. A.; Baron, G. V.; Snurr, R. Q. Langmuir 2004, 20, 150.

(236) Lu, L.; Wang, Q.; Liu, Y. J. Phys. Chem. C 2005, 109, 8845.

(237) Lu, L.; Lu, X.; Chen, Y.; Huang, L.; Shao, Q.; Wang, Q. Fluid Phase Equilib. 2007, 259, 135.

(238) Lachet, V.; Boutin, A.; Tavitian, B.; Fuchs, A. H. Faradav Discuss. 1997, 106, 307

(239) Lachet, V.; Boutin, A.; Tavitian, B.; Fuchs, A. H. Langmuir 1999, 15,8678 .

(240) Talbot, J. AIChE J. 1997, 43, 2471.

(241) Krishna, R.; Baur, R. Sep. Purif. Technol. 2003, 33, 213.
(242) Denayer, J. F. M.; De Meyer, K.; Martens, J. A.; Baron, G. V. Angew. Chem., Int. Ed. 2003, 42, 2774.

(243) Krishna, R.; van Baten, J. M. Chem. Eng. J. 2008, 140, 614.

(244) Funke, H.; Argo, A.; Falconer, J.; Noble, R. Ind. Eng. Chem. Res. 1997, 36, 137.

(245) Yu, M.; Falconer, J. L.; Noble, R. D. Langmuir 2005, 21, 7390.

(246) Uguina, M. A.; Sotelo, J. L.; Rodriguez, A.; Gomez-Civicos, J. I.; Lazaro, J. J. Sep. Purif. Technol. 2006, 51, 72.

(247) Schenk, M.; Calero, S.; Maesen, T. L. M.; Benthem, L. L. v.; Verbeek, M. G.; Smit, B. Angew. Chem., Int. Ed. 2002, 41, 2500.

(248) Schenk, M.; Calero, S.; Maesen, T. L. M.; Vlugt, T. J. H.; van Benthem, L. L.; Verbeek, M. G.; Schnell, B.; Smit, B. J. Catal. 2003, $214,88$.

(249) Cottier, V.; Bellat, J. P.; SimonotGrange, M. H.; Methivier, A. $\underline{\text {. }}$ Phvs. Chem. B 1997, 101, 4798.

(250) Tuan, V. A.; Falconer, J. L.; Noble, R. D. Microporous Mesoporous Mater. 2000, 41, 269.

(251) Beerdsen, E.; Dubbeldam, D.; Smit, B.; Vlugt, T. J. H.; Calero, S. J. Phvs. Chem. B 2003, 107, 12088.

(252) Karger, J.; Ruthven, D. Diffusion in Zeolites and other Microporous Solids; Wiley: New York, 1992.

(253) Talu, O.; Sun, M. S.; Shah, D. B. AIChE J. 1998, 44, 681.

(254) Jobic, H.; Karger, J.; Krause, C.; Brandani, S.; Gunadi, A.; Methivier, A.; Ehlers, G.; Farago, B.; Haeussler, W.; Ruthven, D. M. Adsorpt.-J. Int. Adsorpt. Soc. 2005, 11, 403.

(255) Sholl, D. S. Acc. Chem. Res. 2006, 39, 403.

(256) Dubbeldam, D.; Snurr, R. Q. Mol. Simul. 2007, 33, 305.

(257) Jobic, H.; Theodorou, D. N. Microporous Mesoporous Mater. 2007, $102,21$.

(258) Jobic, H.; Karger, J.; Bee, M. Phvs. Rev. Lett. 1999, 82, 4260.

(259) Sanborn, M. J.; Snurr, R. Q. Sep. Purif. Technol. 2000, $20,1$.

(260) Skoulidas, A. I.; Sholl, D. S.; Krishna, R. Langmuir 2003, 19, 7977.

(261) Krishna, R.; van Baten, J. M. Chem. Eng. Sci. 2008, 63, 3120.

(262) Hoogenboom, J. P.; Tepper, H. L.; van der Vegt, N. F. A.; Briels, W. J. J. Chem. Phys. 2000, 113, 6875.

(263) Tepper, H. L.; Briels, W. J. J. Chem. Phvs. 2002, 116, 9464.

(264) Maginn, E.; Bell, A.; Theodorou, D. J. Phys. Chem. 1993, 97, 4173.

(265) Heffelfinger, G. S.; Vanswol, F. J. Chem. Phvs. 1994, 100, 7548.

(266) Thompson, A. P.; Ford, D. M.; Heffelfinger, G. S. J. Chem. Phvs. 1998, 109, 6406 .

(267) Arya, G.; Chang, H. C.; Maginn, E. J. J. Chem. Phvs. 2001, 115, 8112.

(268) Forester, T.; Smith, W. J. Chem. Soc. Faradav Trans. 1997, 93, 3249.

(269) Ghoriai, P.; Yashonath, S.; Lynden-Bell, R. Mol. Phvs. 2002, 100, 641.

(270) Mosell, T.; Schrimpf, G.; Brickmann, J. J.Phvs. Chem. B 1997, 101, 9476.

(271) Mosell, T.; Schrimpf, G.; Brickmann, J. J. Phys. Chem. B 1997, 101, 9485.

(272) Mosell, T.; Schrimpf, G.; Hahn, C.; Brickmann, J. J. Phvs. Chem. 1996, 100,4571

(273) Mosell, T.; Schrimpf, G.; Brickmann, J. J. Phvs. Chem. 1996, 100, 4582.

(274) June, R. L.; Bell, A. T.; Theodorou, D. N. J. Phvs. Chem. 1991, 95, 8866.

(275) Nagumo, R.; Takaba, H.; Nakao, S. I. J. Phys. Chem. C 2008, 112, 2805.

(276) Nivarthi, S.; McCormick, A.; Davis, H. Chem. Phvs. Lett. 1994, 229 , 297.

(277) Bar, N. K.; Karger, J.; Pfeifer, H.; Schafer, H.; Schmitz, W. Microporous Mesoporous Mater. 1998, 22, 289.

(278) Stallmach, F.; Karger, J.; Krause, C.; Jeschke, M.; Oberhagemann, U. J. Am. Chem. Soc. 2000, 122, 9237.

(279) Tunca, C.; Ford, D. M. Chem. Eng. Sci. 2003, 58, 3373.

(280) Tunca, C.; Ford, D. J. Chem. Phvs. 1999, 111, 2751.

(281) Saravanan, C.; Auerbach, S. M. J. Chem. Phvs. 1997, 107, 8120.

(282) Saravanan, C.; Auerbach, S. M. J. Chem. Phvs. 1997, 107, 8132.

(283) Saravanan, C.; Jousse, F.; Auerbach, S. Phys. Rev. Lett. 1998, 80, 5754.

(284) Saravanan, C.; Auerbach, S. M. J. Chem. Phys. 1999, 110, 11000.

(285) Beerdsen, E.; Smit, B.; Dubbeldam, D. Phys. Rev. Lett. 2004, 93, art. no 248301 .

(286) Dubbeldam, D.; Beerdsen, E.; Vlugt, T. J. H.; Smit, B. J. Chem. Phys. 2005, 122, 224712.

(287) Dubbeldam, D.; Beerdsen, E.; Calero, S.; Smit, B. $\underline{\text { J. Phvs. Chem. B }}$ 2006, 110, 3164.

(288) Barrer, R. M.; Vaughn, D. E. W. Transactions of the Faradav Societv 1971, 67, 2129.

(289) Deem, M. W.; Newsam, J. M.; Creighton, J. A. J. Am. Chem. Soc. 1992, 114, 7198.

(290) Kopelevich, D. I.; Chang, H.-C. J. Chem. Phvs. 2001, 115, 9519. 
(291) Demontis, P.; Fois, E.; Suffritti, G.; Quartieri, S. J. Phvs. Chem. 1990, 95, 4329.

(292) Demontis, P.; Suffritti, G.; Mura, P. Chem. Phvs. Lett. 1992, 191, 553.

(293) Fritzsche, S.; Wolfsberg, M.; Haberlandt, R.; Demontis, P.; Suffritti, G. B.; Tilocca, A. Chem. Phys. Lett. 1998, 296, 253.

(294) Fritzsche, S.; Wolfsberg, M.; Haberlandt, R. Chem. Phvs. 2003, 289, 321.

(295) Leroy, F.; Rousseau, B.; Fuchs, A. H. Phvs. Chem. Chem. Phvs. 2004, 6,775 .

(296) Zimmermann, N. E. R.; Jakobtorweihen, S.; Beerdsen, E.; Smit, B.; Keil, F. J. J. Phvs. Chem. C 2007, 111, 17370.

(297) Millot, B.; Methivier, A.; Jobic, H.; Moueddeb, H.; Dalmon, J. A. Microporous Mesoporous Mater. 2000, 38, 85.

(298) Millot, B.; Methivier, A.; Jobic, H.; Moueddeb, H.; Bée, M. J. Phvs. Chem. B 1999, 103, 1096.

(299) Paschek, D.; Krishna, R. Phvs. Chem. Chem. Phvs. 2000, 2, 2389

(300) Reed, D. A.; Ehrlich, G. Surf. Sci. 1981, 102, 588.

(301) Krishna, R.; Paschek, D.; Baur, R. Microporous Mesoporous Mater. 2004, 76, 233

(302) Krishna, R.; van Baten, J. M. Microporous Mesoporous Mater. 2008, 109, 91.

(303) Jousse, F.; Auerbach, S. J. Chem. Phvs. 1997, 107, 9629.

(304) Jousse, F.; Auerbach, S. M.; Vercauteren, D. P. J. Phys. Chem. B $1998,102,6507$.

(305) Kamat, M.; Keffer, D. Mol. Phys. 2003, 101, 1399.

(306) Paschek, D.; Krishna, R. Chem. Phvs. Lett. 2001, 342, 148.

(307) Skoulidas, A. I.; Sholl, D. S. J. Phvs. Chem. B 2001, 105, 3151.

(308) Kramer, G.; Beest, B. v.; Santen, R. v. Nature 1991, 351, 636.

(309) Jousse, F.; Auerbach, S. M.; Vercauteren, D. P. J. Chem. Phvs. 2000, $112,1531$.

(310) Karger, J.; Demontis, P.; Suffritti, G. B.; Tilocca, A. J. Chem. Phys. 1999, $110,1163$.

(311) Demontis, P.; Karger, J.; Suffritti, G. B.; Tilocca, A. Phys. Chem. Chem. Phvs. 2000, 2, 1455

(312) Demontis, P.; Suffritti, G. B.; Tilocca, A. J. Chem. Phys. 2000, 113, 7588.

(313) Jousse, F.; Leherte, L.; Vercauteren, D. P. J. Phvs. Chem. B 1997, $101,4717$.

(314) Fritzsche, S.; Karger, J. Europhvs. Lett. 2003, 63, 465.

(315) Lopez, F.; Perez, R.; Ruette, F.; Medina, E. Phvs. Rev. E 2005, 72, 061111.

(316) Saravanan, C.; Jousse, F.; Auerbach, S. M. J. Chem. Phvs. 1998 108,2162

(317) Coppens, M. O.; Bell, A. T.; Chakraborty, A. K. Chem. Eng. Sci. 1998, 53, 2053.

(318) Krishna, R.; van Baten, J. M. Microporous Mesoporous Mater. 2008, 107, 296.

(319) Reyes, S. C.; Sinfelt, J. H.; DeMartin, G. J. J. Phvs. Chem. B 2000, 104,5750

(320) Beerdsen, E.; Dubbeldam, D.; Smit, B. Phys. Rev. Lett. 2005, 95, 164505 .

(321) Beerdsen, E.; Smit, B. J. Phvs. Chem. B 2006, 110, 14529.

(322) Li, S. G.; Falconer, J. L.; Noble, R. D.; Krishna, R. J. Phvs. Chem. C 2007, 111, 5075 .

(323) Shah, D.; Guo, C.; Hayhurst, D. J. Chem. Soc., Faraday Trans. 1995, 91, 1143.

(324) Krishna, R.; Vlugt, T.; Smit, B. Chem. Eng. Sci. 1999, 54, 1751.

(325) Catlow, C. R. A.; Freeman, C. M.; Vessal, B.; Tomlinson, S. M.; Leslie, M. J. Chem. Soc., Faradav Trans. 1991, 87, 1947.

(326) Hernández, E.; Catlow, C. Proc. R. Soc. London, Ser. A 1995, 448, 143.

(327) Nicholas, J.; Trouw, F.; Mertz, J.; Iton, L.; Hopfinger, A. J. Phvs. Chem. 1993, 97, 4149.

(328) Raj, N.; Sastre, G.; Catlow, C. R. A. J. Phvs. Chem. B 1999, 103 , 11007.

(329) Corma, A.; Catlow, C.; Chica, A.; Sastre, G. J. Phvs. Chem. B 1998, 102,7085

(330) Sastre, G.; Catlow, C.; Chica, A.; Corma, A. J. Phvs. Chem. B 2000, $104,416$.

(331) Sastre, G.; Catlow, C. R. A.; Corma, A. J. Phys. Chem. B 2002, $106,956$.

(332) Sastre, G.; Corma, A. Top. Catal. 2003, 24, 7.

(333) Fan, J. F.; Wang, Q. X.; Gong, X. D.; Xiao, H. M. THEOCHEM 2003, 638, 129

(334) Leroy, F.; Jobic, H. Chem. Phys. Lett. 2005, 406, 375.

(335) Wongthong, P.; Rungsirisakun, R.; Probst, M.; Limtrakul, J. Microporous Mesoporous Mater. 2007, 100, 160.

(336) Jobic, H.; Schmidt, W.; Krause, C. B.; Karger, J. Microporous Mesoporous Mater. 2006, 90, 299.

(337) Karger, J.; Ruthven, D. M. Zeolites 1989, 9, 267.

(338) Jobic, H.; Theodorou, D. N. J.Phvs. Chem. B 2006, 110, 1964.
(339) Fernandez, M.; Karger, J.; Freude, D.; Pampel, A.; van Baten, J. M.; Krishna, R. Microporous Mesoporous Mater. 2007, 105, 124.

(340) Kawano, M.; Vessal, B.; Catlow, C. J. Chem. Soc. Chem. Commun. $1992,12,879$.

(341) Smirnov, K. Chem. Phvs. Lett. 1994, 229, 250.

(342) Ahunbay, M. G.; Elliott, J. R.; Talu, O. J. Phys. Chem. B 2002, 106, 5163.

(343) Bussai, C.; Fritzsche, S.; Haberlandt, R.; Hannongbua, S. J. Phys. Chem. B 2004, 108, 13347.

(344) Bussai, C.; Fritzsche, S.; Haberlandt, R.; Hannongbua, S. Langmuir $\mathbf{2 0 0 5}, 21,5847$.

(345) Hussain, I.; Titiloye, J. O. Microporous Mesoporous Mater. 2005, $85,143$.

(346) Krishna, R.; van Baten, J. M.; Dubbeldam, D. J.Phvs. Chem. B 2004, 108,14820 .

(347) Krishna, R.; van Baten, J. M. Chem. Phvs. Lett. 2005, 407, 159.

(348) Krishna, R.; van Baten, J. M. Chem. Phvs. Lett. 2006, 420, 545.

(349) Jobic, H.; Laloue, N.; Laroche, C.; van Baten, J. M.; Krishna, R. J. Phvs. Chem. B 2006, 110, 2195.

(350) Chmelik, C.; Heinke, L.; Kaerger, J.; Schmidt, W.; Shah, D. B.; van Baten, J. M.; Krishna, R. Chem. Phys. Lett., in press (DOI: http:// dx.doi.org/10.1016/j.cplett.2008.05.023).

(351) Beerdsen, E.; Dubbeldam, D.; Smit, B. Phvs. Rev. Lett. 2006, 96, 044501.

(352) Leroy, F.; Rousseau, B. Mol. Simul. 2004, 30, 617.

(353) Chong, S. S.; Jobic, H.; Plazanet, M.; Sholl, D. S. Chem. Phvs. Lett. 2005, 408, 157.

(354) Jobic, H. J. Mol. Catal. A: Chem. 2000, 158, 135.

(355) Keffer, D.; Alon, V.; Davis, H. T. Mol. Phvs. 1996, 87, 367.

(356) Webb, E. B. I.; Grest, G. S. Catal. Lett. 1998, 56, 95.

(357) Gorring, R. J. Catal. 1973, 31, 13.

(358) Chen, N.; Garwood, W. E.; Dwyer, F. G. Shape Selective Catalysis in Industrial Applications; Volume 65 of Chemical Industries, 2nd ed.; Marcel Dekker: New York, 1996.

(359) Chen, N.; Lucki, S.; Mower, E. J. Catal. 1969, 13, 329.

(360) Cavalcante, C., Jr.; Eic, M.; Ruthven, D.; Occelli, M. Zeolites 1995, $15,293$.

(361) Magalhes, F. D.; Laurence, R. L.; Conner, W. C. AIChE J. 1996, 42,68 .

(362) Ruckenstein, E.; Lee, P. Phvs. Lett. 1976, 56A, 423.

(363) Derouane, E. G.; Andre, J. M.; Lucas, A. A. J. Catal. 1988, 110, 58.

(364) Nitsche, J. M.; Wei, J. AIChE J. 1991, 37, 661.

(365) Tsekov, R.; Ruckenstein, E. J. Chem. Phvs. 1994, 100, 3808.

(366) Tsekov, R.; Smirniotis, P. G. J. Phys. Chem. B 1998, 102, 9385.

(367) Tsekov, R.; Evstatieva, E. Adv. Colloid Interface Sci. 2005, 114, 159.

(368) Terranova, G.; Aldao, C. M.; Martin, H. O. Phys. Rev. E 2005, 71, art. no. 0211032 .

(369) Ruthven, D. M. J. Phys. Chem. B 2004, 108, 16328.

(370) Ruthven, D. M. Microporous Mesoporous Mater. 2006, 96, 262.

(371) Eic, M.; Ruthven, D. Zeolites 1995, 8, 472.

(372) Jobic, H.; Methivier, A.; Ehlers, G.; Farago, B.; Haeussler, W. Angew. Chem. Int. Ed. 2004, 43, 364.

(373) Kolokolov, D. I.; Arzumanov, S. S.; Stepanov, A. G.; Jobic, H. $\underline{J}$. Phys. Chem. C 2007, 111, 4393

(374) Yoo, K.; Tsekov, R.; Smirniotis, P. G. J. Phvs. Chem. B 2003, 107, 13593.

(375) Bhide, S. Y.; Yashonath, S. J. Am. Chem. Soc. 2003, 125, 7425.

(376) Ghorai, P. K.; Yashonath, S.; Demontis, P.; Suffritti, G. B. J. Am. Chem. Soc. 2003, 125, 7116.

(377) Yashonath, S.; Santikary, P. J. Phvs. Chem. 1994, 98, 6368.

(378) Yashonath, S.; Bandyopadhyay, S. Chem. Phys. Lett. 1994, 228, 284.

(379) Yashonath, S.; Santikary, P. J. Chem. Phvs. 1994, 100, 4013.

(380) Bhide, S. Y.; Yashonath, S. Mol. Phys. 2004, 102, 1057.

(381) Schenk, M.; Smit, B.; Vlugt, T.; Maesen, T. Angew. Chem., Int. Ed. 2001, 40, 736 .

(382) Czaplewski, K. F.; Reitz, T. L.; Kim, Y. J.; Snurr, R. Q. Microporous Mesoporous Mater. 2002, 56, 55.

(383) Levitt, D. Phvs. Rev. A 1973, 8, 3950.

(384) Gupta, V.; Nivarthi, S. S.; McCormick, A. V.; Davis, H. T. Chem. Phvs. Lett. 1995, 247, 596.

(385) Gupta, V.; Nivarthi, S. S.; Keffer, D.; McCormick, A. V.; Davis, H. T. Science 1996, 274, 164.

(386) Hahn, K.; Karger, J.; Kukla, V. Phvs. Rev. Lett. 1996, 76, 2762.

(387) Jobic, H.; Hahn, K.; Karger, J.; Bee, M.; Tuel, A.; Noack, M.; Girnus, I.; Kearley, G. J. J. Phvs. Chem. B 1997, 101, 5834.

(388) Hahn, K.; Kärger, J. J. Phys. Chem. B 1998, 102, 5766.

(389) Demontis, P.; Gonzalez, J. G.; Suffritti, G. B.; Tilocca, A. J. Am. Chem. Soc. 2001, 123, 5069.

(390) Sholl, D.; Fichthorn, K. Phvs. Rev. Lett. 1997, 79, 3569.

(391) Mon, K. K.; Percus, J. K. J. Chem. Phys. 2002, 117, 2289.

(392) Nelson, P. H.; Auerbach, S. M. J. Chem. Phys. 1999, 110, 9235. 
(393) MacElroy, J. M. D.; Suh, S. H. Microporous Mesoporous Mater. 2001, 48, 195

(394) MacElroy, J. M. D.; Suh, S. H. J. Chem. Phvs. 1997, 106, 8595.

(395) Sholl, D. S.; Lee, C. K. J. Chem. Phvs. 2000, 112, 817.

(396) Paschek, D.; Krishna, R. Langmuir 2001, 17, 247.

(397) Paschek, D.; Krishna, R. Phys. Chem. Chem. Phys. 2001, 3, 3185.

(398) Maceiras, D. B.; Sholl, D. S. Langmuir 2002, 18, 7393.

(399) Snurr, R. Q.; Karger, J. J. Phvs. Chem. B 1997, 101, 6469.

(400) Jost, S.; Fritzsche, S.; Haberlandt, R. Chem. Phys. Lett. 1997, 279, 385.

(401) Jost, S.; Bar, N. K.; Fritzsche, S.; Haberlandt, R.; Karger, J. J. Phys. Chem. B 1998, 102, 6375.

(402) Sanborn, M. J.; Snurr, R. Q. AIChE J. 2001, 47, 2032.

(403) Adhangale, P.; Keffer, D. Sep. Sci. Technol. 2003, 38, 977.

(404) Nivarthi, S. S.; McCormick, A. V. J. Phys. Chem. 1995, 99, 4661.

(405) Hong, U.; Karger, J.; Pfeifer, H. J. Am. Chem. Soc. 1991, 113, 4812.

(406) Smit, B.; Krishna, R. Chem. Eng. Sci. 2003, 58, 557.

(407) Krishna, R.; van Baten, J. M. Sep. Purif. Technol. 2007, 55, 246.

(408) Krishna, R.; van Baten, J. M. Chem. Eng. J. 2007, 133, 121.

(409) Newsome, D. A.; Sholl, D. S. J. Phys. Chem. B 2006, 110, 22681.

(410) Gupta, A.; Snurr, R. Q. J. Phvs. Chem. B 2005, 109, 1822.

(411) Arya, G.; Maginn, E. J.; Chang, H. C. J. Phvs. Chem. B 2001, 105 , 2725.

(412) Bigot, B.; Peuch, V.-H. J. Phys. Chem. 1995, 99, 8206.

(413) Vigné-Maeder, F.; Amrani, S.; Gélin, P. J. Catal. 1992, 134, 536.

(414) Webb, E. B.; Grest, G. S. J. Chem. Phvs. 2002, 116, 6311.

(415) Newsome, D. A.; Sholl, D. S. J. Phys. Chem. B 2005, 109, 7237.

(416) Blauwhoff, P. M. M.; Gosselink, J. W.; Kieffer, E. P.; Sie, S. T.; Stork, W. H. J. Zeolites as catalysts in industrial processes. In Catalysis and Zeolites: Fundamentals and Applications; Weitkamp, J., Puppe, L., Eds.; Springer: Berlin, 1999.

(417) Degnan, T. F. J. Catal. 2003, 216, 32.

(418) Marcilly, C. R. Top. Catal. 2000, 13, 357

(419) Tanabe, K.; Földerich, W. Appl. Catal., A 1999, 181, 399.

(420) Weisz, P. B. Microporous Mesoporous Mater. 2000, 35-36, 1.

(421) Weisz, P.; Frilette, V. J. Phvs. Chem. 1960, 64, 382.

(422) Weisz, P. B. Chem. Tech. 1973, 3, 498.

(423) Weisz, P. B. Pure Appl. Chem. 1980, 52, 2091.

(424) Venuto, P. B. Perspectives on zeolite catalysis. In Molecular Sieve Zeolites II; Flanigen, E., Sand, L., Eds.; American Chemical Society: Washington, DC, 1971; Vol. 102.

(425) Chen, N. Y.; Degan, T. F. J.; Smith, C. M. Molecular Transport and Reaction in Zeolites: Design and Application of Shape Selective Catalysts; Wiley: New York, 1994.

(426) Csicsery, S. M. J. Catal. 1971, 23, 124.

(427) Csicsery, S. M. Shape-selective catalysis. In Zeolite Chemistry and Catalysis; Rabo, J., Ed.; American Chemical Society: Washington, DC, 1976; Vol. 171.

(428) Csicery, S. Zeolites 1984, 4, 202.

(429) Csicsery, S. M. Chem. Br. 1985, 21, 473-4.

(430) Csicsery, S. M. Pure Appl. Chem. 1986, 58, 841.

(431) Csicsery, S. M. The future of shape selective catalysis. In Catalysis by Microporous Materials; Beyer, H., Ed.; Elsevier: Amsterdam, 1995; Vol. 94.

(432) Haag, W. O.; Lago, R. M.; Weisz, P. B. Faradav Discuss. 1981, 72, 317.

(433) Weitkamp, J.; Ernst, S.; Puppe, L. Shape-selective catalysis in zeolites. In Catalysis and Zeolites; Weitkamp, J., Puppe, L., Eds.; Springer: Berlin, 1999.

(434) Rabo, J. A.; Pickert, P. E.; Mays, R. L. Ind. Eng. Chem. 1961, 53, 733.

(435) Rabo, J. A.; Bezman, R. D.; Poutsma, M. L. Acta Phvs. Chem. 1978, 24,39 .

(436) Poutsma, M. L.; Schaffer, S. R. J. Phvs. Chem. 1973, 77, 158.

(437) Rabo, J. A. Appl. Catal., A: Gen. 2002, 229, 7.

(438) Rabo, J. A. Catal. Rev.-Sci. Eng. 1981, 23, 293.

(439) Rabo, J. A. Unifying principles in zeolite chemistry and catalysis. In Zeolites: Science and Technology; Ribeiro, F., Rodrigues, A., Rollmann, L., Naccache, C., Eds.; Kluwer: Dordrecht, 1984; Vol. 80.

(440) Rabo, J. A. Speculations on molecular sieve and ionization effects in Y zeolite. In Proceedings of the Sixth International Zeolite Conference; Olson, D., Bisio, A., Eds.; Butterworth-Heinemann: Burlington, MA, 1984.

(441) Rabo, J. A. New directions in molecular sieve science and technology. In Surface Organometallic Chemistry: Molecular Approaches to Surface Catalysis; Basset, J.-M., Gates, B. C., Candy, J.-P., Choplin, A., Leconte, M., Quignard, F., Santini, C., Eds.; Kluwer: Dordrecht, 1988; Vol. 231.

(442) Corma, A.; Wojciechowski, B. W. Catal. Rev.-Sci. Eng. 1985, 27, 29.

(443) Derouane, E. G. Chem. Phvs. Lett. 1987, 142, 200.
(444) Derouane, E. G. Confinement effects in sorption and catalysis by zeolites. In Guidelines for Mastering the Properties of Molecular Sieves; Barthomeuf, D., Derouane, E., Holderich, W., Eds.; Plenum: New York, 1990; Vol. 221.

(445) Derouane, E. G. J. Mol. Catal. A: Chem. 1998, 134, 29.

(446) Rabo, J. A.; Gajda, G. J. Catal. Rev.-Sci. Eng. 1990, 31, 385.

(447) Denayer, J. F.; Baron, G. V.; Souverijns, W.; Martens, J. A.; Jacobs, P. A. Ind. Eng. Chem. Res. 1997, 36, 3242.

(448) Jentys, A.; Lercher, J. A. Techniques of zeolite characterization. In Introduction to Zeolite Science and Practice, 2nd ed.; Bekkum, $\mathrm{H}$. v., Jacobs, P., Flanigen, E., Jansen, J., Eds.; Elsevier: Amsterdam, 2001; Vol. 137.

(449) Narbeshuber, T. F.; Vinek, H.; Lercher, J. A. J. Catal. 1995, 157, 388.

(450) van de Runstraat, A.; van Grondelle, J.; van Santen, R. A. Ind. Eng. Chem. Res. 1997, 36, 3116.

(451) Van Santen, R. A.; De Gauw, F. J. M. M. Acidity in zeolite catalysis. In 12th International Congress on Catalysis; Corma, A., Melo, F., Mendioroz, S., Fierro, J., Eds.; Elsevier: Amsterdam, 2000; Vol. $130 \mathrm{~A}$.

(452) Wei, J. Chem. Eng. Sci. 1996, 51, 2995

(453) Lercher, J. A.; Seshan, K. Curr. Opin. Solid State Mater. Sci. 1997, 2, 57.

(454) Cheng, J. C.; Degnan, T. F.; Beck, J. S.; Huang, Y. Y.; Kalyanaraman, M.; Kowalski, J. A.; Loehr, C. A.; Mazzone, D. N. A comparison of zeolites MCM-22, Beta, and USY for liquid phase alkylation of benzene with ethylene. In Science and Technology in Catalysis; Hattori, H., Otsuka, K., Eds.; Elsevier: Amsterdam, 1999; Vol. 121.

(455) Sastre, G.; Catlow, C. R. A.; Corma, A. J. Phvs. Chem. B 1999, 103,5187

(456) Satterfield, C. N.; Katzer, J. R.; Vieth, W. R. Ind. Eng. Chem. Fundam. 1971, 10, 478.

(457) Derouane, E. G. J. Catal. 1986, 100, 541.

(458) Laxmi Narasimhan, C. S.; Thybaut, J. W.; Marin, G. B.; Martens, J. A.; Denayer, J. F.; Baron, G. V. J. Catal. 2003, 218, 135.

(459) Martens, J. A.; Parton, R.; Uytterhoeven, L.; Jacobs, P. A.; Froment, G. F. Appl. Catal. 1991, 76, 95.

(460) Martens, J. A.; Jacobs, P. A. Some aspects of molecular shapeselective catalysis with hydrocarbons in zeolites. In Zeolite Microporous Solids: Synthesis, Structure, and Reactivity; Derouane, E., Lemos, F., Naccache, C., Ribeiro., F., Eds.; Kluwer: Dordrecht, 1992; Vol. 352.

(461) Claude, M. C.; Martens, J. A. J. Catal. 2000, 190, 39.

(462) Claude, M. C.; Vanbutsele, G.; Martens, J. A. J. Catal. 2001, 203, 213.

(463) Souverijns, W.; Martens, J. A.; Uytterhoeven, L.; Froment, G. F.; Jacobs, P. A. Selective key-lock catalysis in dimethylbranching of alkanes on ton-[barrel]-type zeolites. In Progress in Zeolite and Microporous Materials; Chon, H., Ihm, S.-K., Uh, Y., Eds.; Elsevier: Amsterdam, 1997; Vol. 105B.

(464) Souverijns, W.; Martens, J. A.; Froment, G. F.; Jacobs, P. A. J. Catal. 1998, 174, 177.

(465) Chen, N. Y. Molecular shape-selective hydrocarbon conversion over erionite. In Molecular Sieves, 3rd ed.; Meier, W. M., Uytterhoeven, J. B., Eds.; Leuven University Press: Leuven, 1973.

(466) Santilli, D. S.; Zones, S. I. Catal. Lett. 1990, 7, 383.

(467) Derouane, E. G.; Gabelica, Z. J. Catal. 1980, 65, 486.

(468) Derouane, E. G. J. Catal. 1981, 72, 177.

(469) Santilli, D.; Harris, T.; Zones, S. Microporous Mater. 1993, 1, 329.

(470) Van Nordstrand, R. A.; Santilli, D. S.; Zones, S. I. An all-silica molecular sieve that is isostructural with aluminum phosphate (AlPO4-5). In Perspectives in molecular sieve science; Flank, W. H., Whyte, T. E., Eds.; American Chemical Society: Washington, DC, 1988; Vol. 368.

(471) Van Nordstrand, R. A.; Santilli, D. S.; Zones, S. I. Synth. Microporous Mater. 1992, 1, 373.

(472) Coonradt, H. L.; Garwood, W. E. Ind. Eng. Chem. 1964, 3, 38.

(473) Mills, G. A.; Heinemann, H.; Milliken, T. H.; Oblad, A. G. Ind. Eng. Chem. 1953, 45, 134

(474) Weisz, P. B. Polyfunctional heterogeneous catalysis. In Advances in Catalysis and Related Subjects; Eley, D. D., Frankenburg, W. G., Selwood, P. W., Weisz, P. B., Eds.; Acdemic Press: San Diego, 1962; Vol. 13.

(475) Weitkamp, J. Influence of chain length in hydrocracking and hydroisomerization of n-alkanes. In Hydrocracking and Hydrotreating; Ward, J. W., Qader, S. A., Eds.; American Chemical Society: Washington DC, 1975; Vol. 20.

(476) Weitkamp, J. Erdoel Kohle, Erdgas, Petrochem. 1978, 31, 13.

(477) van Santen, R. A.; Neurock, M. Molecular Heterogeneous Catalysis: A Conceptual and Computational Approach; Wiley-VCH: Weinheim, 2006.

(478) Froment, G. F. Catal. Todav 1987, 1, 455. 
(479) Alvarez, F.; Ribeiro, F. R.; Perot, G.; Thomazeau, C.; Guisnet, M. J. Catal. 1996, 162, 179 .

(480) Martens, G. G.; Marin, G. B.; Martens, J. A.; Jacobs, P. A.; Baron, G. V. J. Catal. 2000, 195, 253.

(481) Martens, G.; Froment, G. F. Kinetic modeling of paraffins hydrocracking based upon elementary steps and the single event concept. In Reaction Kinetics and the Development of Catalytic Processes; Froment, G., Waugh, K., Eds.; Elsevier: Amsterdam, 1999; Vol. 122.

(482) Denayer, J. F. M.; Martens, J. A.; Jacobs, P. A.; Thybaut, J. W.; Marin, G. B.; Baron, G. V. Appl. Catal. 2003, 246, 17.

(483) Thybaut, J. W.; Marin, G. B.; Baron, G. V.; Jacobs, P. A.; Martens, J. A. J. Catal. 2001, 202, 324.

(484) Olken, M. M.; Garces, J. M. Characterization of dealuminated mordenite by $\mathrm{n}$-decane hydroconversion. In Proceedings from the Ninth International Zeolite Conference, Montreal, 1992, Vol. 2; Ballmoos, R. v., Higgins, J. B., Treacy, M. M. J., Eds.; ButterworthHeinemann: Boston, 1993.

(485) Weitkamp, J.; Ernst, S. Factors influencing the selectivity of hydrocracking in zeolites. In Guidelines for Mastering the Properties of Molecular Sieves; Barthomeuf, D., Derouane, E., Holderich, W., Eds.; Plenum: New York, 1990; Vol. 221.

(486) Sie, S. T. Ind. Eng. Chem. Res. 1993, 32, 403.

(487) Brouwer, D. M.; Hogeveen, H. Prog. Phys. Org. Chem. 1972, 9, 179.

(488) Condon, F. E. Catalytic isomerization of hydrocarbons. In Catalysis; Emmett, P., Ed.; Reinhold: New York, 1958; Vol. 6.

(489) Calero, S.; Schenk, M.; Dubbeldam, D.; Maesen, T. L. M.; Smit, B. J. Catal. 2004, 228, 121.

(490) Sastre, G.; Chica, A.; Corma, A. J. Catal. 2000, 195, 227.

(491) Raybaud, P.; Patrigeon, A.; Toulhoat, H. J. Catal. 2001, 197, 98.

(492) Bronsted, N. Chem. Rev. 1928, 5, 231.

(493) Evans, M. G.; Polanyi, M. Trans. Faradav Soc. 1938, 34, 11.

(494) Pieterse, J. A. Z.; Veefkind-Reyes, S.; Seshan, K.; Lercher, J. A. J. Phys. Chem. B 2000, 104, 5715.

(495) Weisz, P. B. Erdoel Kohle 1965, 18, 525.

(496) Du, H.; Olson, D. H. J. Phys. Chem. B 2002, 106, 395.

(497) Corma, A.; Martinez-Soria, V.; Schnoeveld, E. J. Catal. 2000, 192, 163.

(498) Siffert, S.; Gaillard, L.; Su, B. L. J. Mol. Catal. A: Chem. 2000, $153,267$.

(499) Ocakoglu, R. A.; Denayer, J. F. M.; Marin, G. B.; Martens, J. A.; Baron, G. V. J. Phys. Chem. B 2003, 107, 398.

(500) Derouane, E. G.; Nagy, J. B.; Fernandez, C.; Gabelica, Z.; Laurent, E.; Maljean, P. Appl. Catal. 1988, 40, L1.

(501) Derouane, E. G.; Nagy, J. B. Appl. Catal. 1989, 52, 169.

(502) Derouane, E. G.; Gabelica, Z.; Jacobs, P. A. J. Catal. 1981, 70, 238.

(503) Smit, B.; Maesen, T. L. M. Nature 2008, 451, 671.

(504) Maesen, T. L. M.; Calero, S.; Schenk, M.; Smit, B. J. Catal. 2006, 237, 278.

(505) Feijen, E. J. P.; Martens, J. A.; Jacobs, P. A. Isomerization and hydrocracking of decane and heptadecane on cubic and hexagonal faujasite zeolites and their intergrowth structures. In 11th International Congress on Catalysis, 40th anniversary: proceedings of the 11th ICC, Baltimore, MD, June 30-July 5, 1996, Vol. 101; Hightower, J. W., Ed.; Elsevier: Amsterdam, 1996.

(506) Martens, J. A.; Perez-Pariente, J.; Jacobs, P. A. Acta Phys. Chem. 1985, 31, 487

(507) Martens, J. A.; Tielen, M.; Jacobs, P. Relation between paraffin isomerisation capability and pore architecture of large-pore bifunctional zeolites. In Zeolites as Catalysts, Sorbents and Detergent Builders, Applications and Innovations. Proceedings of an International Symposium, Würtzburg, September 4-8, 188, Vol. 46; H. G. Karge, J. Weitkamp Eds.; Elsevier: Amsterdam, 1988.

(508) Martens, J. A.; Vanbutsele, G. M.; Jacobs, P. A. Characterization of large and extra-large zeolite pores with the heptadecane test. In Proceedings from the Ninth International Zeolite Conference, Montreal, 1992, Vol. 2; Ballmoos, R. v., Higgins, J. B., Treacy, M. M. J., Eds.; Butterworth-Heinemann: Boston, 1993.

(509) Martens, J. A.; Uytterhoeven, L.; Jacobs, P. A.; Froment, G. F. Isomerization of long-chain n-alkanes on platinum/HZSM 22 and $\mathrm{Pt} / \mathrm{HY}$ zeolite catalysts and on their intimate mixtures. In New Frontiers in Catalysis: Proceedings of the 10th International Congress on Catalysis, Budapest, July 19-24, 1992, Vol. 75; Guczi, L., Solymosi, F., Teteny, P., Eds.; Elsevier: Amsterdam, 1993.

(510) Martens, J. A.; Jacobs, P. A. J. Mol. Catal. 1993, 78, L47.

(511) Maesen, T. L. M.; Calero, S.; Schenk, M.; Smit, B. J. Catal. 2004, 221, 241.

(512) Jacobs, P. A.; Uytterhoeven, J. B.; Steyns, M.; Froment, G.; Weitkamp, J. Hydroisomerization and hydrocracking. 1. Comparison of the reactions of n-decane over ultrastable $\mathrm{Y}$ and ZSM-5 zeolites containing platinum. In Proceedings of the fifth International Conference on Zeolites, Naples, Italy, 2-6 June 1980; Rees, L., Ed.; Heyden: London, 1980.

(513) Martens, J. A.; Tielen, M.; Jacobs, P. A. Acta Chim. Hung. 1985, $119,203$.

(514) Martens, J. A.; Jacobs, P. A. Conceptual background for the conversion of hydrocarbons on heterogeneous acid catalysts. In Theoretical Aspects of Heterogeneous Catalysis; Moffat, J., Ed.; Van Nostrand Reinhold: Berlin, 1990.

(515) Stull, D. R.; Westrum, E. F. J.; Sinke, G. C. The Chemical Thermodynamics of Organic Compounds; Wiley: New York, 1969.

(516) Bronsted, N. Trans. Faraday Soc. 1928, 712, 726.

(517) Norskov, J. K.; Bligaard, T.; Logadottir, A.; Bahn, S.; Hansen, L. B.; Bollinger, M.; Bengaard, H.; Hammer, B.; Sljivancanin, Z.; Mavrikakis, M.; Xu, Y.; Dahl, S.; Jacobsen, C. J. H. J. Catal. 2002, 209, 275.

(518) Toulhoat, H.; Raybaud, P. J. Catal. 2003, 216, 63.

(519) Rozanska, X.; Santen, R. A. V. Reaction Mechanism in Zeolite Catalysis. In Handbook of Zeolite Science and Technology; Auerbach, S. M., Carrado, K. A., Dutta, P. K., Eds.; Marcel Dekker Inc.: New York, 2003.

(520) Maesen, T. L. M.; Schenk, M.; Vlugt, T. J. H.; Smit, B. J. Catal. 2001, 203, 281 .

(521) Jacobs, P.; Martens, J.; Weitkamp, J.; Beyer, H. Faraday Discuss. Chem. Soc. 1981, 72, 353.

(522) Weitkamp, J.; Jacobs, P. A.; Martens, J. A. Appl. Catal. 1983, 8, 123.

(523) Aerts, A.; Huybrechts, W.; Kremer, S. P. B.; Kirschhock, C. E. A.; Theunissen, E.; Van Isacker, A.; Denayer, J. F. M.; Baron, G. V.; Thybaut, J. W.; Marin, G. B.; Jacobs, P. A.; Martens, J. A. Chem. Commun. 2003, 15, 1888.

(524) Persson, A. E.; Schoeman, B. J.; Sterte, J.; Otterstedt, J. E. Zeolites 1994, 14, 557.

(525) Persson, A. E.; Schoeman, B. J.; Sterte, J.; Otterstedt, J. E. Zeolites 1995, 15,611

(526) Schmidt, I.; Madsen, C.; Jacobsen, C. J. H. Inorg. Chem. 2000, 39, 2279.

(527) Constable, F. H. Proc. R. Soc. (London) 1925, 108A, 355.

(528) Cremer, E. Z. Phys. Chem. 1929, 144, 231.

(529) Cremer, E.; Schwab, G. Z. Phys. Chem. 1929, 144, 243.

(530) Schwab, G. M. Z. Phys. Chem. 1929, 5, 406.

(531) Bond, G. C.; Keane, M. A.; Kral, H.; Lercher, J. A. Catal. Rev. Sci. Eng. 2000, 42, 323.

(532) Wei, J. Ind. Eng. Chem. Res. 1994, 33, 2467.

(533) Chen, N. Y.; Schlenker, J. L.; Garwood, W. E.; Kokotailo, G. T. J. Catal. 1984, 86, 24.

(534) Chen, N.; Garwood, W. Ind. Eng. Chem. Proc. Des. Dev. 1978, 17, 513-.

(535) Denayer, J. F.; Baron, G. V.; Jacobs, P. A.; Martens, J. A. Phys. Chem. Chem. Phys. 2000, 2, 1007.

(536) Domokos, L.; Lefferts, L.; Seshan, K.; Lercher, J. A. J. Catal. 2001, 203, 351.

(537) Martens, J.; Souverijns, W.; Verrelst, W.; Parton, R.; Froment, G.; Jacobs, P. Angew. Chem., Int. Ed. Engl. 1995, 34, 2428.

(538) Greensfelder, B. S. Adv. Chem. Ser. 1951, No. 5, 3.

(539) Greensfelder, B. S.; Voge, H. H.; Good, G. M. J. Ind. Eng. Chem. 1949, 41, 2573.

(540) Rosenbaum, J. M.; Howell, R. L. European Patent Application No. 1037956, 1999.

(541) Duhoux, E.; Germaine, G. R. B.; Sajad Hussein, Y.; Smithers, J. M.; Steenge, W. D. E.; Wedlock, D. J. European Patent Application No. 1791931,2006

(542) Murphy, W. J.; Soled, S. L.; Cody, I. A.; Larkin, D. W.; Helton, T. E.; McVicker, G. B. U.S. Patent Application No. 2006/0073962, 2006.

(543) Benazzi, E.; Guillon, E.; Martens, Y. European Patent Application No. 2004/0290680, 2004.

(544) Maesen, T. L. M.; Beerdsen, E.; Smit, B. U.S. Patent Application 2007/0029229, 2007.

(545) Zones, S.; Burton, A.; Maesen, T.; Smit, B.; Beerdsen, E. U.S. Patent Application 2007/0284284, 2007.

(546) Newsome, D. A.; Sholl, D. S. Microporous Mesoporous Mater. 2008, 107, 286.

(547) Earl, D. J.; Deem, M. W. Ind. Eng. Chem. Res. 2006, 45, 5449.

(548) Thomas, J.; Klinowski, J. Angew. Chem. Int. Ed. 2007, 46, 7160.

(549) Wu, M. G.; Deem, M. W. J. Chem. Phys. 2002, 116, 2125. 